DOE/NV/25946--850

\title{
WORKER ENVIRONMENT BERYLLIUM CHARACTERIZATION STUDY
}

\author{
Prepared by \\ National Security Technologies, LLC \\ Environment, Safety, Health \& Quality \\ P.O. Box 98521 \\ Las Vegas, NV 89193-8521
}

December 2009

\author{
Prepared for the \\ U.S. Department of Energy \\ National Nuclear Security Administration \\ Nevada Site Office \\ Environment, Safety, and Health Division \\ P.O. Box 98518 \\ Las Vegas, NV 89193-8518 \\ WORK PERFORMED UNDER \\ CONTRACT NO. DE-AC52-06NA25946
}




\section{DISCLAIMER}

Reference herein to any specific commercial product, process, or service by trade name, trademark, manufacturer, or otherwise, does not necessarily constitute or imply its endorsement, recommendation, or favoring by the United States Government or any agency thereof or its contractors or subcontractors.

Available for sale to the public from-

\section{U.S. Department of Commerce}

National Technical Information Service

5301 Shawnee Road

Alexandria, VA 22312

Telephone: 800.553.6847

Fax: 703.605.6900

E-mail: orders@ntis.gov

Online Ordering: http://www.ntis.gov/help/ordermethods.aspx

Available electronically at http://www.osti.gov/bridge

Available for a processing fee to the U.S. Department of Energy and its contractors, in paper, from-

\section{U.S. Department of Energy}

Office of Scientific and Technical Information

P.O. Box 62

Oak Ridge, TN 37831-0062

Telephone: 865.576 .8401

Fax: 865.576.5728

E-Mail: reports@adonis.osti.gov 


\section{APPROVALS}

\section{Original signed by Angela P. Colarusso}

Angetar. Colarusso - $l$

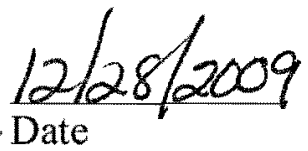

Assistant Manager for Site Operations

U.S. Department of Energy

National Nuclear Security Administration

Nevada Site Office

\section{Original signed by Gerhardt R. Griess}

Gerhardt R. Griess

Director, Environment, Safety, Health \& Quality

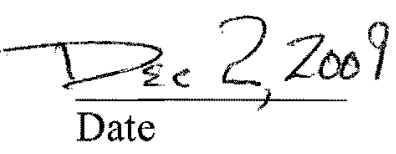

National Security Technologies, LLC 
THIS PAGE INTENTIONALLY LEFT BLANK 


\section{WORKER ENVIRONMENT BERYLLIUM CHARACTERIZATION STUDY}

\section{Purpose}

This report summarizes the conclusion of regular monitoring of occupied buildings at the Nevada Test Site (NTS) and North Las Vegas (NLV) facility to determine the extent of beryllium (Be) contamination in accordance with Judgment of Needs (JON) 6 of the August 14, 2003 "Minnema Report.”

\section{Executive Summary}

From March 2002, when the U. S. Department of Energy, National Nuclear Security Administration Nevada Site Office (NNSA/NSO) was notified that an employee at NLV had been diagnosed with Chronic Beryllium Disease (CBD), to present (October 2009), periodic monitoring of occupied buildings at the NTS and NLV has been performed for the potential introduction or spread of Be, until the extent of contamination could be determined and controls implemented.

During this period, 228 facilities were sampled with over 11,000 surface wipe samples, along with numerous air samples obtained and analyzed. Also during this period, statistical methods of data collection, analysis, and detail were developed and sharpened to provide a high degree of confidence in all results (see Appendices 1 through 4).

All but five of the facilities examined now do not exceed the U.S. Department of Energy (DOE) release criterion (RC) of 0.2 micrograms $(\mu \mathrm{g}) / 100$ square centimeters $\left(\mathrm{cm}^{2}\right)$. Of those five, two have been demolished and the other three involve legacy equipment (not in use) or long-term storage.

\section{Background}

CBD Discovery and DOE National Nuclear Security Administration Headquarters (NNSA/HQ) Investigation

In March 2002, NNSA/NSO was notified that an employee at the NLV facility had been diagnosed with CBD. An NNSA/HQ investigation was chartered on August 22, 2002. The NNSA/HQ team presented its findings in "Investigation of Beryllium Exposure Cases Discovered at the North Las Vegas Facility of the National Nuclear Security Administration" (August 14, 2003), otherwise referred to as the "Minnema Report," named after team leader Douglas Minnema.

The Minnema Report concluded that there was reason to believe that all cases of CBD and Be sensitization likely resulted from exposure to Be during DOE-sponsored activities while working 
on a characterization study at a facility on the NTS, exposure while working in the B-Complex at NLV, and/or exposure while working elsewhere in the DOE Complex (p. viii). The report makes specific recommendations regarding treatment of the suspect buildings (24-B01, -02, and -03 and the second floor of Building A01); see the Building Recommendation Letter (Attachment 2 of the Minnema Report). Subsequently, Buildings B01 and B02 have been demolished, while Building B03 and the second floor of Building A01 have been renovated, and have passed the Recommended Cleanup Standard stated in the Building Recommendation Letter.

Section 4 of the Minnema Report contains 18 JONs. In particular, JON 4 states that "NSO needs to ensure that the full extent of the spread of beryllium contamination (including NTS, NLV, and offsite locations) is determined, and that mitigative or corrective actions are established as appropriate." Furthermore, JON 6 states that "NSO needs to ensure that occupied buildings at NTS and NLV are regularly monitored for the potential introduction and spread of beryllium into uncontrolled areas, at least until the extent of the beryllium contamination has been determined and evaluated and controls have been identified and implemented.”

\section{NNSA/NSO Facility Survey}

In response to these JONs, NNSA/NSO embarked on a broad characterization of Be surface concentrations beginning with the suspect B-Complex buildings while still occupied and extending throughout NLV, the NTS, and ancillary facilities in North Las Vegas, Los Alamos, Livermore, Special Technologies Laboratory in Santa Barbara, and the Andrews Air Force Base. Sampling of both touchable surfaces and air was conducted in occupied and incidentally occupied facilities during 2002-2004. Supplementary chemical analyses continued until early 2007, accompanied by statistical analyses of the resulting data. Some follow-up sampling took place in February 2008 and January 2009. This document reports the conclusions of the sampling and statistical analyses. EnviroStat personnel were actively involved in all stages of this survey from initial discussions with the NNSA/HQ team through sample planning, quality assurance activities, data analysis, and report preparation.

\section{Draft DOE Technical Standard}

While this survey was proceeding, NNSA/HQ and DOE were responding to JON 16, which calls on DOE to provide additional guidance on the implementation of the pertinent federal regulation (Title 10 Code of Federal Regulations [CFR] Part 850). Some of this has resulted in a draft DOE Technical Standard SAFT-0103, "Management of Items and Areas Containing Low Levels of Beryllium.” The Beryllium Health and Safety Committee (BH\&SC) has been active in investigating the many technical and scientific issues involved. EnviroStat personnel have been participating with the BH\&SC as well. 


\section{Structure of the Survey}

Two hundred twenty-eight facilities were sampled during this survey. These facilities range in size from one-room guard stations to large office buildings; they range in nature from office buildings to dormitories, shops, storage facilities, laboratories, and more; and they range in locations from the NTS forward areas to urban settings and air force bases. Over 11,000 surface wipe samples have been obtained and analyzed, along with numerous air samples. There exists a quasi-regulatory standard for surface sampling, and surface Be is ubiquitous in nearly all facilities surveyed; accordingly, the survey was designed for surface sampling. Air monitoring was also conducted while the sampling teams were in the facilities; very few of the air samples produced measurements above the laboratory reporting limit (RL) and none were above the regulatory limit. This report addresses only the surface sampling campaign and conclusions.

\section{DOE Release Criterion}

As discussed in Appendix 1, the quasi-regulatory standard used is the DOE RC of $0.2 \mu \mathrm{g} / 100 \mathrm{~cm}^{2}$ given in 10 CFR 850.31(b)(1) as the limit not to be exceeded on items to be released to non-Be operations or the public. That regulation provides for an exemption for naturally occurring Be; there is evidence that naturally occurring Be, even Be ores, are nontoxic. A critical feature is the fact that analysis of wipe samples for total Be is routine and inexpensive, whereas determining the source and/or speciation (i.e., chemical binding and so on) of the Be in a given sample can be expensive. Therefore, a guiding principle of this survey has been the following:

- Survey the facility for total Be. If the facility passes, there is nothing more to be done.

- If the facility does not pass when considering the total Be, then attempt to show that the Be present is natural as opposed to anthropogenic.

- Alternately, or if the Be present may be anthropogenic, clean the facility and resample.

With the acquisition in late 2005 of its inductively coupled plasma mass spectroscopy (ICP-MS) instrument, National Security Technologies, LLC (NSTec), now has the ability to attempt to distinguish Be from native NTS soils and anthropogenic Be.

\section{$\underline{\text { Survey Design and Evolution }}$}

Appendix 1 lays out the general strategy to be employed in this survey, and outlines discussions of some of the evolutionary enhancements added since the survey was begun in late 2002. Appendices 3 and 4 discuss in detail several issues that arose and their resolutions. A few of the major points follow. 
Types of Facilities and Statistical Comparisons

Facilities fall into one of four categories:

- Facilities consisting of just one or a few Individual Work Areas (IWAs), all of which have been sampled, such as a guard station or tunnel access control station;

- Facilities consisting of multiple IWAs, too numerous to reasonably sample, such as an office building or laboratory;

- Facilities consisting of one or more open areas (Areas), such as a warehouse or shop; or

- Facilities having portions consisting of both IWAs and Areas, such as the NTS cafeteria and several shop facilities.

In the IWA cases, samplers were instructed to sample four to six locations within the IWA most likely to produce high measurements. For example, in a typical cubicle, one might select corners behind computer monitors, tops of wall dividers, or tops of computers located under desktops. If the largest value found in the IWA is no greater than the RC, the IWA itself passes. If all IWAs in a facility are sampled and pass, the facility as a whole passes.

Where there are more IWAs in a facility than can reasonably be sampled, a statistically based random selection of IWAs is made. The IWA maximum for each sampled IWA is found, and the resulting data are compared statistically with the RC. Three statistical criteria are used. The most stringent (for all but the smallest facilities) is termed the PLall approach: one uses the IWA maxima to compute a 95\%-prediction limit (PL) for the maximum value that would be found in all remaining IWAs if they were also sampled. If PLall is not greater than the RC (and no individual measurement exceeds the RC), the facility passes. The PLall approach was suggested to the NNSA/HQ investigation team for use with the suspect NLV buildings, and was enthusiastically endorsed by that team.

As discussed in Appendix 1, this approach is considerably more stringent than that contained in the draft DOE Technical Standard. That document prefers a statistical comparison based on 95\%95\% upper tolerance limits (UTLs), which are upper 95\% confidence limits for the $95^{\text {th }}$ percentile of the distributions of all measurements (not just the IWA maxima). Moreover, it contemplates random sampling of surfaces rather than the biased sampling described above. Accordingly, two less stringent criteria are also used in this report. One is a UTL based on the IWA maxima (see Appendix 1). Both PLall and UTL are computed for virtually all IWA facilities; so long as at least one of these is not greater than the RC (and no individual observation exceeds the RC), the facility passes. The third criterion is termed UTL_cluster. This criterion uses all data, not just the IWA maxima. The idea is that selecting IWAs and then sampling locations within IWAs is similar to "cluster sampling" in survey research methodology. The mathematics underlying this UTL method is nonstandard; see Appendix 4 for its development. Again, if the UTL_cluster is not greater than the RC (and no individual measurement exceeds the RC), the facility passes. This UTL cluster analysis approach is still 
more stringent than that advocated in the draft DOE Technical Standard because of the biased sampling used.

For Area facilities, a standard grid sampling plan is laid out on floors, walls, and/or shelves as appropriate to the facility, and the UTL is computed from the resulting data. This corresponds to the process anticipated in the draft DOE Technical Standard.

Finally, for facilities with both IWA and Area portions, the same techniques and comparisons are used, with confidence levels adjusted to ensure that the overall confidence for the facility is no less than the target $95 \%$.

\section{Chemical Analysis and Data Issues}

The first 5,000+ wipe analyses were performed by DataChem (DC), an independent, American Industrial Hygiene Association-accredited subcontract laboratory. After that, Bechtel Nevada (BN) acquired its own inductively coupled plasma atomic emission spectroscopy instrument to analyze the majority of the remaining wipe samples obtained during 2003-2004. As a quality control precaution, a number of side-by-side wipes were obtained to allow comparison of the DC and BN results. For a few facilities, data from samples previously obtained and analyzed by Assay Technology's (AT’s) laboratories were used.

The comparison of side-by-side results was not favorable, particularly with respect to samples obtained in shop and forward areas. An extended study of the causes of the observed discrepancies ensued. The results of this study are discussed in Appendix 3; see also Davis et al. (2005). The bottom line is that the particular emission line used in the BN atomic emission spectroscopy (BN-AES) analyses is subject to significant interferences from iron. Appendix 3 presents an adjustment that can be used so long as the iron interference is not too great; the resulting data are termed "BNadj” measurements.

Once that obstacle had been overcome, there remained around 1,000 samples with excessive iron interference. By that time, BN had acquired a new ICP-MS instrument. The digested wipes from the BN-AES analyses had been archived; most were reanalyzed using the BN-MS instrument. These digested samples had been held long past their usual holding time limits, however, and the analytical results were systematically notably higher than those obtained from DC analyses of side-by-side samples obtained for quality assurance. This presented a quandary.

Many of the samples reanalyzed by MS were from the Area 6 shops, which is not surprising since the iron concentrations should naturally be higher in facilities where metal work is conducted. It cannot be determined whether the higher MS values are indeed due to higher Be values, to holding time complications, or both.

The following strategy was adopted to deal with this quandary. For any individual sample location (including its side-by-side comparison location), if a DC and/or BNadj measurement 
was available, it (the higher, if there are both) was used. Failing that, the MS_arch value was used for that location, resulting in statistical analyses likely to be yet more conservative than UTL or PLall analyses using, say, DC data, which are already more stringent than the analyses discussed in the draft DOE Technical Standard. (These high MS_arch values were a significant motivation for the development of the UTL cluster analysis methodology.)

A number of facilities did not pass using this conservative strategy. These were resampled during January 2009 (February 2008 for Area 06-908), with wipes analyzed by ICP-MS within holding time limits. These data are denoted "MS_new." The statistical analyses were repeated using the MS_new data in place of the MS_arch data. For a few facilities both MS_arch and MS_new data were used.

There remain a small number of facilities for which even this latest approach was unsuccessful. In anticipation of this eventuality, bulk samples had been obtained inside the facilities sampled during January 2009, and exterior soil samples were obtained as well. These bulk and soil samples were analyzed for Be concentration (in $\mu \mathrm{g} / \mathrm{kilogram).} \mathrm{In} \mathrm{addition,} \mathrm{concentrations} \mathrm{of}$ other metals were measured in these samples and in the wipe samples with Be exceeding the DOE RC. The bulk sample Be concentrations were then compared with the range of values found in NTS soils, and the metal concentration ratios for both bulk and wipe samples were compared with the range found in NTS soils. In only six cases was there suspicion that the Be present in the samples was not from NTS soils; see the discussion in the "Survey results" section to follow.

Also, in a few cases, a facility would not pass if all data were included, but would if one or two "outlier" measurements were excluded. In these cases, an "outlier" involves both an atypically high value for that facility and a sampling location that arguably would not fit the criterion of being "touchable" during normal work activities.

\section{Statistical Issues}

Another issue has to do with the nature of the measurement data obtained. The conventional wisdom is that measurements of concentrations of environmental contaminants tend to have lognormal distributions; that is, the logs of the data have normal (Gaussian) distributions. There is some theoretical justification for this, along with considerably regulatory acceptance. Moreover, this conventional wisdom is not inconsistent with our data.

The distributions of measurements of those concentrations are more interesting, however. At the beginning of these investigations, the NNSA/HQ team very strongly urged following the Multi-Agency Radiation Survey and Site Investigation Manual guidance. One recommendation of that guidance is that uncensored data be obtained whenever possible; that is, one obtains and uses actual instrument measurement values for all analyses rather than allowing low measurements to be reported as "nondetects" or “ $<$ RL” for some RL, as is conventionally done. Having such a large number of uncensored observations has turned out to be highly educational, and revises our view of the conventional wisdom stated above. This is a major topic explored in 
Davis et al. (2005), cited previously; see also Davis and Grams (2006) and Davis, Field, and Gran (2009).

It turns out that at some facilities a high proportion of data values are negative, which is impossible under the lognormal model. It has been necessary to develop a reasonable accommodation for such data; this accommodation is described in Appendix 3. Essentially, a small constant is added to the reported values to make them all positive, lognormal UTL or PLall analyses are conducted, and the constant is subtracted from the resulting limit. Appendix 3 discusses the technical issues in detail, including incorporating blank corrections to alleviate low-level discrepancies in measurements obtained using different measurement systems and in determining appropriate constants for the various measurement systems and facility types.

For a few facilities the available data are censored, either at a convention RL (for AT data) or at 0 (for some early DC data, due to a temporary flaw in database design). In these cases lognormal censored data statistical methods are used; see Davis (2006).

\section{Survey Results}

The following table summarizes the results of the survey.

\begin{tabular}{|lcccc|}
\hline & Summary of Results & & & \\
Facility Type & Total & Pass & Pass* & Fail \\
\hline Offices, Quarters, Labs, Communications, Experimentation Facilities & 71 & 67 & 4 & $\mathbf{0}$ \\
Field Operations & $\mathbf{8 7}$ & 77 & $\mathbf{8}$ & 2 \\
Shops & 47 & 35 & $\mathbf{1 1}$ & $\mathbf{1}$ \\
Storage & 23 & $\mathbf{1 6}$ & $\mathbf{5}$ & 2 \\
\hline All & $\mathbf{2 2 8}$ & $\mathbf{1 9 5}$ & $\mathbf{2 8}$ & $\mathbf{5}$ \\
\hline * Facilities which pass when outliers are disregarded and/or Be bulk concentrations and metal ratios are \\
considered. \\
\end{tabular}

Two of the five facilities that failed have been demolished. Of the others, one is a very small facility (one IWA) in Area 6; one in Area 23 has a single isolated high value on a floor swipe with a slightly low Yttrium to Be ratio (although other metal ratios are consistent with those in NTS soils); and one in Area 6 has Be on a machine tool. Detailed results by facility are given on pages 3-8 of Appendix 2.

\section{Summary and Discussion}

A large-scale survey of worker environments has been conducted at facilities under the responsibility of NNSA/NSO, triggered by the Be exposure incident reported in March 2002. That survey included measurements of both removable Be concentrations on surfaces and air Be concentrations obtained. Two hundred and twenty-eight facilities were surveyed in all, mostly located on the NTS and in NLV, but also including operations in Los Alamos, Livermore, and Andrews Air Force Base. 
Of the 228 surveyed facilities, 93 are small, consisting of one to three IWAs. Sampling was based on the sampler's judgment about where the highest Be concentrations were likely to be found. If the IWA maximum value does not exceed the DOE RC of $0.2 \mu \mathrm{g} / 100 \mathrm{~cm}^{2}$, the IWA is "clean.” If all IWAs in a facility are clean, the facility "passes.”

For larger facilities, a statistically designed sampling of IWAs or Areas was conducted. UTLs or PLs for the IWA maxima of all unsampled IWAs (PLalls) were computed and compared with the RC. A facility passes if at least one of these limits is no greater than the RC and no individual measurement exceeds the RC. For IWA facilities, this criterion is more stringent than that suggested in the draft DOE Technical Standard; accordingly, another UTL cluster analysis was developed to more nearly match that draft DOE Technical Standard.

Of the 228 surveyed facilities, 195 facilities pass the comparison with the RC. Eleven additional facilities would pass if isolated high measurements obtained at atypical locations were disregarded; an "atypical” location is one not touched during normal work activities, such as a door top or the top of an electrical box.

These conclusions are based on analysis of wipe samples for total Be, as discussed previously. This includes both naturally occurring and anthropogenic Be, whereas the relevant regulation clearly exempts Be from natural soils. The analysis for total Be is straightforward and inexpensive, whereas determining the source of the Be in a given sample is likely to be more challenging; moreover, there is clear guidance in the draft DOE Technical Standard for the comparisons regarding total Be, but the guidance is not yet so clear for the latter sorts of comparisons. Nonetheless, with the acquisition in 2005 of the ICP-MS instrument, NNSA/NSO and NSTec now have the capability of performing the sort of chemical speciation needed to support discussions of the likely origin of Be in facilities showing higher levels. In 17 of the remaining facilities, Be concentrations in bulk samples and metal ratios in bulk and wipe samples are quite consistent with those in NTS soils.

In five facilities none of the criteria allow the facility to "pass." Two of these facilities have been demolished. The others have been cleaned to below the release criteria.

Finally, JON 6 of the Minnema Report calls for regular monitoring of occupied buildings at the NTS and NLV "at least until the extent of the beryllium contamination has been determined and evaluated and controls have been identified and implemented.” In this survey, 90\% of facilities were found to have minimal surface total Be levels satisfying the DOE RC. Be concentrations in bulk samples and metal ratios in bulk and wipe samples show that the Be found in another 7\% of facilities clearly resembles that found in NTS soils, which is not intended as “contamination” by the pertinent regulation. The very few exceptions identified or possibly identified involve legacy equipment or long-term storage. 


\section{Conclusion}

Occupied buildings at NLV and the NTS have been regularly monitored for the introduction or spread of Be since 2002 with no apparent increase in contamination. All 18 JONs associated with the Minnema Report have now been completed or fulfilled. There remains only three facilities with any indication of Be over the release criteria, and those are known and controlled. It is appropriate to cease Be survey activities in areas outside identified legacy and environmental restoration operations.

\section{References}

Box, G. E. P., 1979, “Robustness in the Strategy of Scientific Model-Building,” Robustness in Statistics, R. Launer and G. Wilkenson, eds., Academic Press.

Code of Federal Regulations, Title 10, Part 850, “Chronic Beryllium Disease Prevention Program,” 2003.

Code of Federal Regulations, Title 40, Part 136, Appendix B, "Guidelines Establishing Test Procedures for the Analysis of Pollutants,” 2008.

Davis, C. B., 2004, Planning Sample Sizes for Normal Distribution Based Upper Tolerance and Prediction Limits (unpublished technical report).

Davis, C. B., 2005, "Planning a Beryllium Characterization Sampling Campaign for a DOE Facility” (unpublished technical report).

Davis, C. B., 2006, "Parametric 95\%-95\% Upper Tolerance Limits for Censored Lognormal Data,” presented at the Joint Statistical Meetings, Seattle, WA.

Davis, C. B., and N. E. Grams, 2006, “When Laboratories Should Not Censor Analytical Data, and Why,” presented at the EPA’s 25th Annual Conference on Managing Environmental Quality Systems, Austin, TX.

Davis, C. B., and R. J. McNichols, 1987, “One-Sided Intervals for at Least $p$ of $m$ Observations from a Normal Population on Each of $r$ Future Occasions,” Technometrics 29, 359-370.

Davis, C. B., and R. J. McNichols, 1999, “Simultaneous Nonparametric Prediction Limits” (with discussion and response), Technometrics 41, 89-112.

Davis, C. B., D. E. Field, and T. E. Gran, 2009, “A Model for Measurements of Lognormally Distributed Environmental Contaminants,” presented at the Joint Statistical Meetings, Washington, D.C. 
Davis, C. B., D. E. Field, J. W. Hess, and D. A. Jensen, 2005, "What is a Beryllium Measurement? A Critical Look at Beryllium Quantitation,” presented at the Second Symposium on Beryllium Particulates and Their Detection, Salt Lake City, UT.

Deubner, D., M. Kelsh, M. Shum, L. Maier, M. Kent, and E. Lau, 2001, "Beryllium Sensitization, Chronic Beryllium Disease, and Exposures at a Beryllium Mining and Extraction Facility,” Applied Occupational and Environmental Hygiene 16, 579-592.

Grams, N. E., and C. B. Davis, 2009, "Data Use, Quality, Reporting, and Communication,” Chapter 8 of Beryllium: Environmental Analysis and Monitoring, M. J. Brisson and A. A. Echechukwu, eds, The Royal Society of Chemistry.

Minnema, D. M., 2003, "Investigation of Beryllium Exposure Cases Discovered at the North Las Vegas Facility of the National Nuclear Security Administration,” U.S. Department of Energy, National Nuclear Security Administration, Washington, D.C.

Rocke, D. M., and S. Lorenzato, 1995, "A Two-Component Model for Measurement Error in Analytical Chemistry,” Technometrics 37, 176-184.

Sansonetti, J. E., and W. C. Martin, 2005, Handbook of Basic Atomic Spectroscopic Data, National Institute of Standards and Technology, http://physics.nist.gov/PhysREFData/ Handbook/index.html.

Wegner, R., R. Heinrich-Ramm, D. Nowak, K. Olma, B. Poschadel, and D. Szadkowski, 2000, "Lung function, biological monitoring, and biological effect monitoring of gemstone cutters exposed to beryls," Occupational and Environmental Medicine 57, 133-139. 


\section{Appendix 1 DATA QUALITY OBJECTIVES AND STATISTICAL METHODS}

This appendix is largely excerpted from the document "Planning a Beryllium Characterization Sampling Campaign for a DOE Facility,” prepared in August 2005. It reflects the approach adopted in structuring the beryllium (Be) sampling conducted by Bechtel Nevada (BN) and, subsequently, by National Security Technologies, LLC, at the U.S. Department of Energy (DOE), National Nuclear Security Administration (NNSA) North Las Vegas facility (NLV), Nevada Test Site (NTS), and ancillary facilities. It is not necessarily the approach that will ultimately appear in the draft DOE Technical Standard SAFT-0103, "Management of Items and Areas Containing Low Levels of Beryllium.” The reader may wish to contact David Weitzman (David.Weizman@hq.doe.gov) for updated information on the direction in which the draft DOE Technical Standard is headed.

The BN approach evolved since the earliest days of the Be investigations at selected buildings in NLV in the late summer of 2002. Some of that history is discussed in what follows. On the whole, though, this document describes a reasonable approach for NLV, NTS, and similar sites, based on the knowledge and experience gained since beginning these investigations. [Sections in reduced type size discuss modifications added during the course of this extended investigation.]

Those first steps in 2002 were influenced by three factors: (a) a perceived urgency of the situation due to possible adverse publicity along with the desire to relocate workers promptly from a possibly unhealthy environment, (b) guidance from the DOE Headquarters (HQ) investigation team that emphatically suggested following the Multi-Agency Radiation Survey and Site Investigation Manual (MARSSIM) to the extent possible, and (c) an initial uncertainty about whether exposure limits based on the Title 10 Code of Federal Regulations (CFR) Part 850 release criterion (RC) are in fact adequately protective of worker health.

Investigations by $\mathrm{BN}$ and $\mathrm{DOE} / \mathrm{HQ}$ teams arrived at the following conclusions. With regard to (a), BN's screening of individual work areas (cubicles, offices, restrooms, etc.) and personal computers in the suspect NLV workplaces gave no indication of any health hazard exposure to employees working in the areas of initial concern. The BN investigation was subsequently expanded to cover all occupied and "incidentally occupied" worker environments at NTS, NLV, and ancillary facilities. The results of these investigations are provided in detail in Appendix 2. As for (b), the MARSSIM document is quite useful in that it has been accepted by the DOE, U.S. Environmental Protection Agency (EPA), and other federal agencies, and it does offer generally appropriate advice on sampling for these sorts of situations. However, it is designed for radiological screening using a combination of wipe samples to ensure that average surface concentrations do not exceed acceptable levels, augmented by gamma or other scans to detect hot spots that may exist. The latter hot-spot scans are not available for Be and many other metals; nonetheless the operative regulation suggests that all workers be protected, not just workers on average. The MARSSIM guidance must, therefore, be adapted to the needs of these surveys. 
With regard to (c), the NNSA investigation team concluded that there is no reason to require a lower regulatory threshold for wipe sample concentrations than the 10 CFR 850 RC. Moreover, discussions in the Beryllium Health and Safety Committee (BH\&SC) reveal that it is not at all clear what role surface contamination actually plays in chronic beryllium disease (CBD) or sensitization. The latter consideration is irrelevant with respect to compliance with current regulation, although one may well recognize that the facility survey decision criteria are based on an analogy of the regulatory requirement regarding the free release of equipment for use by the public, which has commonly been adopted for the facility survey setting.

This document is organized around the seven steps of the EPA's Data/Decision Quality Objectives (DQO) process.

\section{DQO Step 1. State the problem.}

Be in metallic form is a key component in nuclear devices. It is also a valuable alloying material for other metals. Therefore, it is often found at NNSA facilities; in particular, Be has undoubtedly been scattered during device detonations at the NTS. Be is also toxic. The major problem comes from inhaling airborne particles; the major adverse consequence is CBD, an incurable debilitating lung ailment. There is a theory, not universally accepted, that CBD can be acquired in two stages, the first being sensitization and the second being an adverse reaction to subsequent exposure. Under this theory, a person can become sensitized to Be through dermal contact as well as inhalation, but acquiring CBD itself requires inhalation. This theory warrants controlling surface concentrations.

Exposure limits for Be workers are contained in Title 10 Code of Federal Regulations (CFR) Part 850, the federal regulation driving DOE and other Be investigations. In 10 CFR 850, worker exposure is defined as exposure to airborne Be of a worker not wearing respiratory protective equipment. The 10 CFR 850 action level is 0.2 micrograms per cubic meter $\left(\mu \mathrm{g} / \mathrm{m}^{3}\right)$ (§850.23(a)), evaluated as an 8-hour time-weighted average exposure measured in a worker's breathing zone by personal air monitoring. Accordingly, ambient air sampling is a key component of all Be investigations. Very few air samples obtained in occupied facilities or incidentally occupied facilities (see the discussion of terms that follows) produced measurements above their detection limits of approximately $0.007 \mu \mathrm{g} / \mathrm{m}^{3}$. Hence, no formal sampling plans have been created for air sampling; air sampling is accomplished as an adjunct to formal surface sampling conducted under the sampling plans described in this document.

Be workers must be provided with protective clothing and equipment when airborne concentrations exceed the $0.2-\mu \mathrm{g} / \mathrm{m}^{3}$ action level or when surface concentrations exceed $3 \mu \mathrm{g} / 100 \mathrm{~cm}^{2}$ of removable contamination, under $\$ 850.29$. Surfaces contaminated with Be-containing dust and wastes must not exceed $3 \mu \mathrm{g} / 100 \mathrm{~cm}^{2}$ of removable contamination during nonoperational periods, per $\$ 850.30$. However, there is no similar standard in the regulation for non-Be workers. 
The closest thing in the regulation to a surface concentration limit for non-Be workers is the RC for releasing equipment to non-Be facilities or the general public, stated in §850.31(b)(1). The $\mathrm{RC}$ is $0.2 \mu \mathrm{g} / 100 \mathrm{~cm}^{2}$ of removable concentration, or "the concentration level ... in the soil at the point [of] release, whichever is greater." The latter clause is difficult to interpret literally since soil concentrations would be volume concentrations ( $\mu$ g per unit volume) rather than surface concentrations ( $\mu \mathrm{g}$ per unit area); the intent appears to be that naturally occurring Be concentrations from native soils should be excluded from this rule. This RC has generally been adopted as the threshold for surface concentrations in non-Be worker environments, although, strictly speaking, it was not promulgated as such. There are currently no Be workers at the NTS, NLV, or ancillary facilities.

[Added July 2009: Some DOE sites have elected to rely primarily on the alternate volumetric concentration comparison rather than the surface concentration comparison, particularly in dusty settings. A logical extension of the intent of that regulation is to evaluate not only volumetric concentrations of $\mathrm{Be}$, but also the ratios of $\mathrm{Be}$ with other metals found in the environment, the chemical "fingerprint" of the sample. The draft DOE Technical Standard begins to address the volumetric comparison issue, though in a rudimentary form at this point in time; it does not address the more sophisticated fingerprint comparison. Ultimately, the NTS investigation relied on all of these in evaluating the dustiest of facilities.]

As stated previously, the RC was derived solely from housekeeping considerations and is not based on health risk. Hence, it is not clear that there is a risk of adverse affect from dermal exposure to Be-containing dust. If not, the pathway for an adverse health effect from surface contamination would involve resuspension, which was not detected in air sampling during BN's investigations of occupied and incidentally occupied facilities.

Be in the worker environment could come from a variety of sources:

- soils native to the NTS and NLV,

- surface contamination from historical site activities tracked into these facilities from field workers,

- residual contamination remaining from historical Be machining operations in various locations at NTS and NLV, and

- residual contamination from machining alloys of copper and aluminum containing minor proportions of Be.

Since Be occurs naturally in soils at the NTS and in NLV, that source should in principle be considered to be in a separate class from the others, although discriminating between natural and anthropogenic Be sources is more involved than simply measuring surface concentrations of Be. [The newly-acquired capability to perform fingerprinting provides some capabilities along these lines (July 2009).] On the other hand, even if the source is naturally occurring soil, one could argue that improved housekeeping would be in order for worker protection, regardless of the intent of the particular regulation involved, although there does appear to be evidence that naturally occurring $\mathrm{Be}$ presents much less risk to workers exposed, if any risk at all; see, for example, Wegner et al. (2000) and Deubner et al (2001). 
Therefore, the bottom line for DQO Step 1 is to attempt to ensure that all workers at the NTS, $\mathrm{NLV}$, and ancillary facilities are not exposed to removable surface contamination exceeding the 0.2 micrograms $(\mu \mathrm{g}) / 100$ square centimeters $\left(\mathrm{cm}^{2}\right) \mathrm{RC}$. If surface concentrations for total Be (both natural and anthropogenic) do not exceed the RC, there is no more to do. If some concentrations do exceed the RC, or the situation falls into a gray area in DQO terms (see the discussion to follow), it may be appropriate to attempt to identify the source of the Be. If it is naturally occurring, no remedy other than routine housekeeping may be needed; otherwise, one may need to take other action to avoid worker exposure.

\section{DQO Step 2. Identify the decision.}

Virtually every occupied and incidentally occupied facility at the NTS, NLV, and ancillary facilities was systematically scheduled for removing Be surface contamination. An individualized sampling plan was generated for each facility. The sample data have been analyzed statistically with a goal of demonstrating compliance with the RC.

In general, the areas sampled have been categorized one of two ways: as Individual Work Areas (IWAs) or as open areas (Areas). IWAs are cubicles and small offices, parts of large offices, other work areas, and restrooms. Areas are often warehouses or other storage areas. Either or both may be found in a given facility. If a particular facility consists almost entirely of IWAs, any Areas present may be divided into several IWAs for convenience; for example, a classroom in an office building may be divided into octants (say), and each treated as an IWA.

It is impossible in any statistical analysis to claim 100\% certainty. Therefore, several approaches to demonstrating compliance have been considered and implemented. These begin with the upper tolerance limit (UTL) approach, used for Areas, and the protective limit (L) approach, used for IWAs; details of both are provided in this appendix.

The UTL approach follows current industrial hygiene (IH) practices and the draft DOE Technical Standard. In this approach, the goal is to show with high confidence that the $95^{\text {th }}$ percentile of the distribution of Be surface concentrations is no greater than the RC; doing so is a surrogate for demonstrating that "all workers" are protected. The UTL approach is used for Areas, generally with conventional systematic sampling plans, and occasionally with simple random sampling. Details and formulas are provided subsequently in this appendix.

The UTL approach could be used with IWAs as well. During our initial investigations of the suspect NLV facilities, however, a more stringent PL approach was proposed. This approach received enthusiastic endorsement from the DOE/HQ Investigation Team. The PL approach differs from the UTL approach in several key ways. First, a scientific random selection of IWAs is made from a population list of all IWAs. In sampling the IWAs, however, the IH teams are instructed to swipe the locations judged most likely to provide elevated Be concentrations. This is consistent with IH training and motivation, but is contrary to the usual statistical approach in 
which one would attempt to specify sampling locations randomly. As an example, in a typical cubicle the locations most likely to provide elevated concentrations of Be might be corners behind computer monitors, tops of wall dividers, or tops of computers located under desktops. In an office trailer, they might be those locations as well as windowsills. The sampling teams were instructed to limit attention to locations touchable during normal work operations, however; ventilation ducts, tops of elevated wall fixtures, and surfaces above ceilings or inside walls were off limit, for example.

In this approach, any IWA in which the highest measurement obtained by such biased sampling is not greater than the RC is considered to "pass." Where a small facility consists of only one or a few IWAs and all were sampled, with the highest value being no greater than the RC, the facility as a whole is considered to "pass."

Otherwise, in the PL approach, the goal is to show with high statistical confidence that, based on data obtained from the IWAs selected for sampling, the highest measurement in all remaining unsampled IWAs would not exceed the RC. The PL approach was proposed and adopted before the BH\&SC began its deliberations regarding the DOE Technical Standard, and is considerably more stringent than the UTL approach for all but the smallest facilities.

Given the draft DOE Technical Standard recommendation suggestion of using UTLs, along with the greater stringency of the PL approach, it became desirable to back away from the PL approach in favor of the UTL approach for some facilities. When applied to IWA sampling as described here, our UTL approach implementation is still be more stringent than the UTL approach described in the draft DOE Technical Standard; see the discussion to follow.

[Added December 2007: This was the original formulation of the decision problem. As discussed in Appendix 2, this UTL approach applied to IWA maxima is still more stringent than that of the draft DOE Technical Standard. Accordingly, another less stringent, UTL cluster analysis approach has been identified and implemented for certain IWA facilities as well; that is described briefly below and in detail in Appendix 4.]

Both approaches are described in this document. With either approach, the decision is made separately for each facility. If the attempt to demonstrate compliance with the RC is successful, and if no individual measurement at a facility exceeds the RC, that facility is deemed to be clean.

On the other hand, if any individual measurement exceeds the RC, an additional investigation at that facility follows along with appropriate action. In this case the appropriate action might depend on the apparent source of the excess Be concentrations (natural soil or otherwise).

The third possibility is that neither of the above conclusions can be made based on the initial sampling; this is the gray area referred to above. This would occur when all observations are below the RC, but the statistical confidence that the $95^{\text {th }}$ percentile (in the UTL approach) or the highest measurement that would be obtained in the remaining unsampled IWAs (in the PL approach) is beneath the RC and is not adequately high. In this case, one of two paths can be followed. One path is to evaluate the number of additional samples that should be taken in order 
to demonstrate compliance with the RC, obtain the additional samples, and repeat the decision process, possibly iterating as needed. The other is to look first at the apparent source of the higher Be concentrations in the samples already obtained; if these are naturally occurring, a simple recommendation regarding housekeeping can be made and the investigation concluded for that facility. (In several cases, it turns out that the UTL or PL is high due to sampling an isolated location that does not quite fit the "touchable during normal work operations" description. An option in these cases is to simply note the unusual sample circumstance, then repeat the UTL or PL computation with the unusual data value omitted.) [Added August 2009: Alternatively, the data could be reanalyzed using the less stringent UTL cluster analysis approach, recalling that this is still more conservative than the UTL approach anticipated in the draft DOE Technical Standard.]

“Adequately high confidence” is interpreted as 95\% confidence for each facility, in accordance with common IH practices and draft DOE Technical Standard recommendations.

\section{DQO Step 3. Identify inputs needed to make decisions. DQO Step 4. Define the boundaries of the study.}

Decisions are made separately for each facility. The large majority of facilities are single buildings. In some cases a group of similar buildings, such as attached warehouses or adjacent dormitories, is treated as a single facility, so long as the environments and perceived likelihood of exposure are homogeneous. Conversely, separate areas of a single building having distinctly different uses and, hence, possibly different exposures might be considered to be separate facilities; an example might be a building housing ordinary offices and laboratories along with shops and storage areas.

The MARSSIM provides suggestions regarding the physical sizes of survey units (SUs) about which separate decisions should be made. Areas that are known to be or have been contaminated (possibly before being remediated) are in Class 1. Class 1 SUs should be no larger than 100 square meters $\left(\mathrm{m}^{2}\right)\left(\sim 1076\right.$ square feet $\left.\left(\mathrm{ft}^{2}\right]\right)$ in floor area. Areas that do not fit this description, but cannot be cleared a priori based on available information are designated as Class 2. Class 2 SUs should be no larger than $1000 \mathrm{~m}^{2}\left(\sim 10,764 \mathrm{ft}^{2}\right)$ in floor area. Other areas are designated as Class 3, for which there is no floor area maximum. We consider all facilities to be Class 3, based on current experience along with a sampling strategy (discussed below) that dictates a reasonably uniform sampling of larger facilities. MARSSIM does not impose or suggest minimum sample sizes for SUs, but rather contains a lengthy discussion similar to that provided below. Note, however, that the MARSSIM discussion revolves around inferences for the population median $\left(50^{\text {th }}\right.$ percentile), whereas our inferences will be for the $95^{\text {th }}$ percentile in the UTL approach and something different under the PL approach; hence the MARSSIM sample size recommendations do not apply in this situation.

Sampling plans for Areas generally involve a systematic random sampling of floor, wall, and shelf or other horizontal surface areas. Areas out of reach during normal work activities, such as ceilings, tops of ceiling tiles, transoms, and tops of elevated wall-mounted fixtures are excluded from consideration, as these are not typically available for exposure through dermal contact. 
Samples are taken at assigned locations located on approximately rectangular or triangular grids, designated on engineering drawings of the facilities. The number of samples to be taken is based on both a minimum sample size needed to be reasonably likely to satisfy the RC using the UTL approach on a first sampling round (see subsequent discussion) and a minimum distance between sample locations (in general no more than $20 \mathrm{ft}$ between floor locations and comparably along walls). The former criterion does not vary with the facility size; the latter ensures comprehensive coverage of larger facilities.

Sampling plans for IWAs are somewhat nontraditional as is the data treatment. There is a tension between typical statistical requirements and common IH sampling practices. The IH practice is to sample the locations within a "unit" (whatever that may be) most likely to be contaminated, whereas the statistical requirement typically demands random selection of locations. The procedure adopted here combines these approaches. The IWAs to be sampled are selected essentially randomly, with some thought to balancing the representation among building wings, floors, activities, etc. (a concession to systematic sampling). If a building houses disparate types of operations, but not so disparate as to be considered to contain separate facilities, the sampling plan is biased toward those types of operations more likely to contain removable contamination.

[A historical note: Stratified sampling plans were used in the initial surveys of the suspect buildings in NLV, with heavier sampling in IWAs near air ducts in which detectable Be had been found. At that time, the validity of the RC was under suspicion, so a temporary criterion only one-tenth as high $\left(0.02 \mu \mathrm{g} / 100 \mathrm{~cm}^{2}\right)$ was used. This temporary criterion is uncomfortably close to "detection limits" for the standard analytical methods; moreover, values above that value were and are routinely detected in ambient dust. Therefore, this temporary criterion was taken in the MARSSIM sense; that is, as a criterion for average concentrations, and the comparison with the temporary criterion was based on upper confidence limits for the median. The statistical methodology for doing so with stratified samples is readily available. Stratified sampling does not lend itself to analyses via either the UTL or PL approaches, however, so these data are treated simply as a possibly conservatively biased sampling of IWAs in those facilities for comparisons with the current RC using those approaches.]

Within each selected IWA the sampling team was instructed to sample the locations most likely to contain removable Be contamination, typically the dustiest locations. One sample was obtained in general from each quadrant, possibly with one or two more at locations of the sampler's choosing. All samples were analyzed; the largest measured concentration in the IWA (the IWA maximum) is used as the data value for that IWA. This process results in a conservative assessment compared with random sampling of locations within the IWA. Of course, actually laying out a truly random surface sampling plan within each of the thousands of IWAs to be sampled, or even within the facilities containing those IWAs, would be extremely tedious and difficult to enforce.

As with Area sampling, samplers were instructed to restrict their attention to locations available to dermal contact in ordinary work operations. The BN experience suggests that certain locations are particularly likely to have somewhat higher removable Be surface concentrations: tops of doors, tops of door-closing mechanisms, and other wall-mounted fixtures such as electrical boxes. It is not clear that these should be included in the desired range of locations that represent typical worker exposure. 
Again, as with Area sampling, the sampling plan was laid out on an engineering drawing of the facility as well as on a spreadsheet. Some facilities consist mostly of IWAs with a few open areas, such as a firehouse or a building that is primarily an office building but with a few laboratories, storage areas, or classrooms. In such cases, for convenience the open areas were divided up into subareas that were treated as additional IWAs.

Additional inputs needed include the anticipated distributions of data from typical facilities, the decision rule, and the desired probability of coming to the desired conclusion (no further action needed) on the basis of the initial sampling. (The probability of deciding that a facility satisfies the RC when it does not is implicitly set at 5\% when the conventional 95\% confidence level is adopted for the UTLs.) The costs of the exercise were considered to be of relatively minor interest, since Be analyses are relatively inexpensive and the sampling was carried out by BN personnel.

Finally, these surveys were carried out first in regularly occupied facilities. After that, “incidentally occupied” facilities were surveyed. Incidentally occupied facilities are those in which workers are present only occasionally, such as legacy facilities used occasionally for training, certain storage facilities, and certain remote facilities used only occasionally during experiments or exercises.

\section{DQO Step 5. Develop a decision rule.}

In the UTL approach, the decision for a facility is made using an upper 95\% confidence limit for the $95^{\text {th }}$ percentile of the distribution of surface concentration measurements in the accessible worker environment. These are known as 95\%-content, 95\%-confidence UTLs (95\%-95\% UTLs, or just UTLs). The UTL is compared with the RC. If it is below the RC (and no individual measurement exceeds the RC), the facility is considered clean. This is equivalent to a statistical test rejecting the null hypothesis that the $95^{\text {th }}$ percentile is not below the RC, using a 5\% significance level statistical test. The measurements used are the individual wipe measurements, when using the UTL approach for Areas, or the IWA maxima. If a facility is treated as containing both types of areas, higher confidence levels (1- $\alpha_{1}$ and $1-\alpha_{2}$ with $\left.\alpha_{1}+\alpha_{2}=0.05\right)$ are used for the two types, so that the overall significance level remains $5 \%$ or less. Usually the significance level for the Area portion is set to $1 \%$ and that for the IWA portion is set to $4 \%$, due to the greater stringency of the latter. [Added July 2009: When the IWA_cluster approach is employed, as discussed below and in Appendix 4, significance levels for the two portions are generally set equal to $2.5 \%$, since that approach is not so much more stringent than the UTL approach of the draft DOE Technical Standard.] The significance levels used for the separate portions of the facility are included in the detail result listings in Appendix 2.

In the PL approach, the IWA maxima from the selected and sampled IWAs are used to compute a 95\% PL (PLall) for all IWA maxima that might possibly be obtained in future sampling. The PLall is compared with the RC in the same fashion as in the UTL approach. 
If the UTL or PLall along with all individual measurements are no greater than the RC, the facility is considered to be clean. If not, the next step depends on the data. If there is a measurement above the RC, an investigation and cleanup are undertaken. The investigation focuses on areas in the facility having higher measurements, and may also investigate the apparent source (natural soils or otherwise) of the contamination as discussed previously.

Otherwise, the 95\% percentile of the data is below the RC but both UTL and PLall are above, and no individual measurements exceed the RC. In this case, one may compute a supplementary sample size, obtain further data, and repeat the decision process. The August 2005 document, "Planning a Beryllium Characterization Sampling Campaign for a DOE Facility," contains tables and charts that may be used in sample-size planning. Alternately, one may investigate the apparent source of the higher observations found in the initial sampling, or possibly just clean the facility and resample. As stated above at the conclusion of DQO Step 2, one option is to note an isolated high value at a non-touchable location and then repeat the UTL or PL calculation ignoring that isolated high value.

\section{DQO Step 6. Specify limits on the probabilities of making decision errors.}

Since the use of 95\%-95\% UTLs is well established in IH practice, the significance level of the statistical test for the UTL approach is $5 \%$ by default. This is the probability of deciding "clean" when the facility is actually "dirty"; it is adopted for the PLall approach as well. The complementary risk, of deciding in the initial sampling round that the facility is or may be "dirty" (i.e., does not meet the RC) when it is actually "clean,” is in principle up to BN. One would like to keep it as low as possible consistent with reasonable sampling cost. A reasonable target for this probability would be $5-10 \%$ or so. On the other hand, failing to demonstrate compliance with the RC in the initial sampling is not necessarily the end of the process; if the data are border line, there is the possibility of augmenting the sample and repeating the decision process. This possibility was in fact exercised at a few facilities, even during the initial sampling.

\section{DQO Step 7. Optimize the design.}

\section{$\underline{\text { Prior ideas about the distributions of Be measurements }}$}

What remains then is to decide on how many observations to take to be reasonably likely to come to the correct decision (clean, with no further action needed) at a typical clean facility; these are then spread around Areas or IWAs as described previously. To do this we need some prior ideas about the nature of the distributions of all measurements (for Areas) or IWA maxima (for IWAs).

Data from comparable facilities in NLV had been obtained as early as the summer of 2002 and from the NTS nearly as long ago. Following one of the MARSSIM recommendations, uncensored measurement values for nearly all observations have been obtained, and the reporting limits (RLs) that would typically be used to censor the data have been given separately. That is, 
for nearly all samples we are provided with actual instrument readings, even if negative, rather than data down to a certain level and below that just a " $<$ RL" or equivalent notation. Typical RLs for our analyses would be around $0.004-0.01 \mu \mathrm{g} / 100 \mathrm{~cm}^{2}$.

[A technical note: The stated RLs are based on applying the usual 40 CFR 136 Appendix B algorithms to spiked reagent water samples. The relevance of these to detection and quantitation of wipe samples is not at all clear. Moreover, those algorithms are currently under a court-ordered reevaluation due to uncertainties concerning their scientific validity. Finally, determinations of RLs and quantitation limits are based in principle on statistical discussions of the interpretation of individual measurements, whereas in studies such as these the appropriate criterion should be based on the validity of the decision for a facility as a whole. For these reasons, we were happy to comply with the specific urging of the DOE/HQ investigation team that the MARSSIM admonition to obtain fully quantitated data whenever possible be followed. This and related issues have been the focus of the Data Reporting Task Force of the BH\&SC; see also the discussions by Davis, Field, and Gran (2009) and Grams and Davis (2009).]

Environmental contamination data are very often modeled as having lognormal distributions. That is, the (natural) logs of the measurements are taken as having normal ("Gaussian” or "bell-shaped curve”) distributions. This model generally serves adequately for actual contaminant concentrations, but for the measurements themselves there is an added measurement error component that becomes prominent and eventually dominant at low concentrations. This added measurement error component often results in negative reported concentrations. These cannot be used as such in the standard analyses of log normally distributed data as one can take logarithms of only positive values. Accordingly, some special treatment of low values is needed in order to apply typical lognormal distribution statistical techniques. One such treatment, used here, is to artificially censor the data at some positive value. The actual cutoff value to be used ultimately is still under consideration; for purposes of the following discussions, we use a cutoff value of $0.002 \mu \mathrm{g} / 100 \mathrm{~cm}^{2}$, which is $1 \%$ of the RC. For plotting purposes, all negative values are plotted at zero on the original scale. Censored data normal distribution maximum likelihood estimation algorithms are used for estimating means and standard deviations of the data value logs.

[Added July 2009: The preceding paragraph describes the approach used for planning purposes. Further research, however, determined that this approach was rather excessively conservative, in that it tends to make it difficult to decide that a clean facility really is clean; see discussions by Davis, Field, and Gran (2009). A different treatment was ultimately selected for implementation; see Appendix 3. The conventional lognormal distribution assumption necessarily distorts the actual data distributions when those include high proportions of values near and below zero.]

Figure 1 on the following page shows the pooled distribution for all IWA maxima from the suspect NLV buildings. These data have some selection bias due to the stratified sampling plan used, but as it turned out the stratification was at best only mildly correlated with the results obtained. Figure 2 shows the pooled distribution for all IWA maxima from seven larger buildings containing mostly offices, located in Areas 6 and 23 at the NTS. In both cases, the 


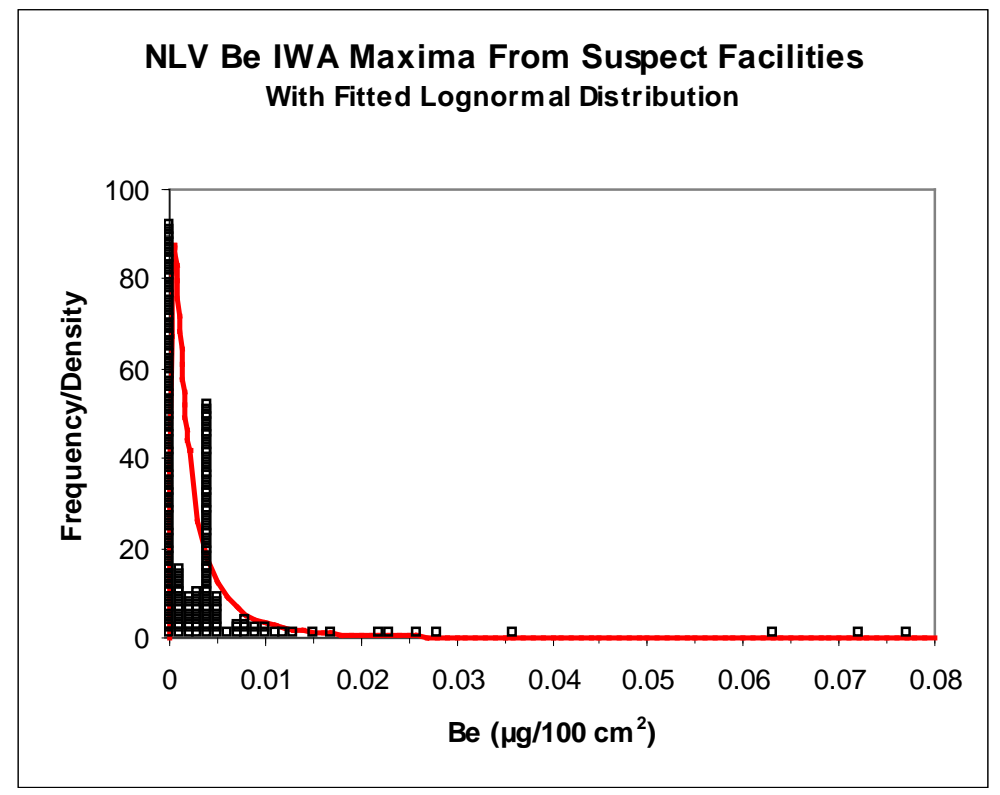

Figure 1: Distribution of IWA maxima from suspect facilities in NLV, with a fitted lognormal distribution. The lognormal distribution parameters are $\mu=-6.2468$ and $\sigma=1.1873$.

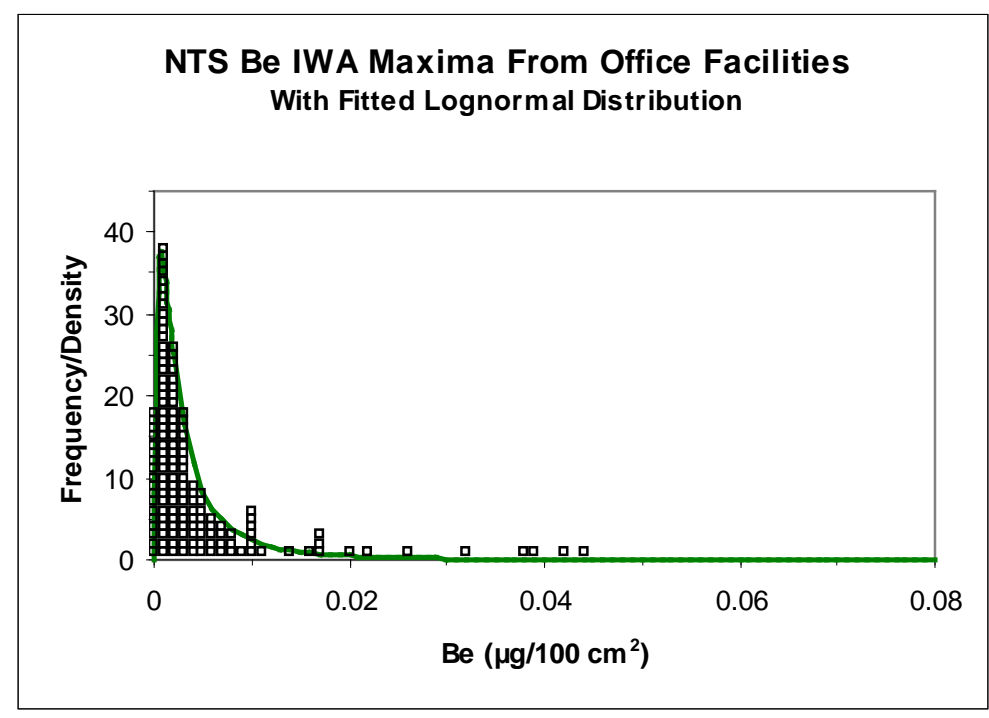

Figure 2: Distribution of IWA maxima from office facilities at the NTS, with a fitted lognormal distribution. The lognormal distribution parameters are $\mu=-5.9775$ and $\sigma=1.1197$. 


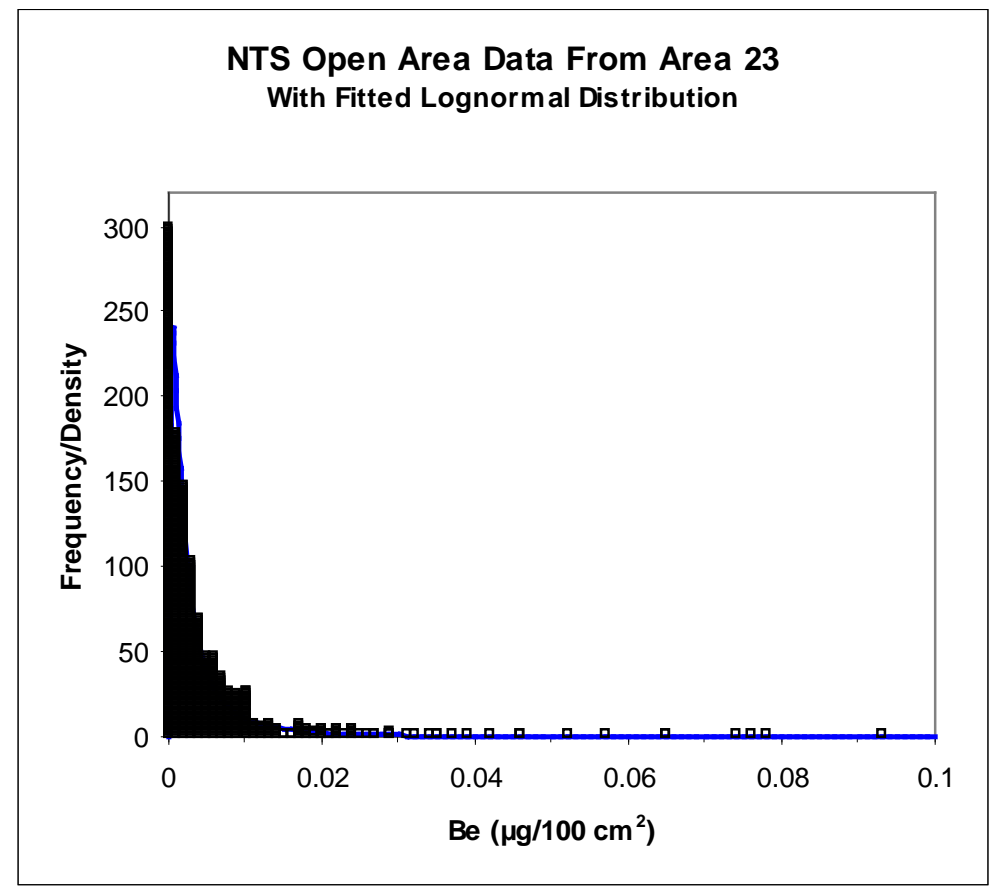

Figure 3: Distribution of data values from Area facilities in Area 23 of the NTS, with a fitted lognormal distribution. The lognormal distribution parameters are $\mu=-6.1497$ and $\sigma=1.2098$.

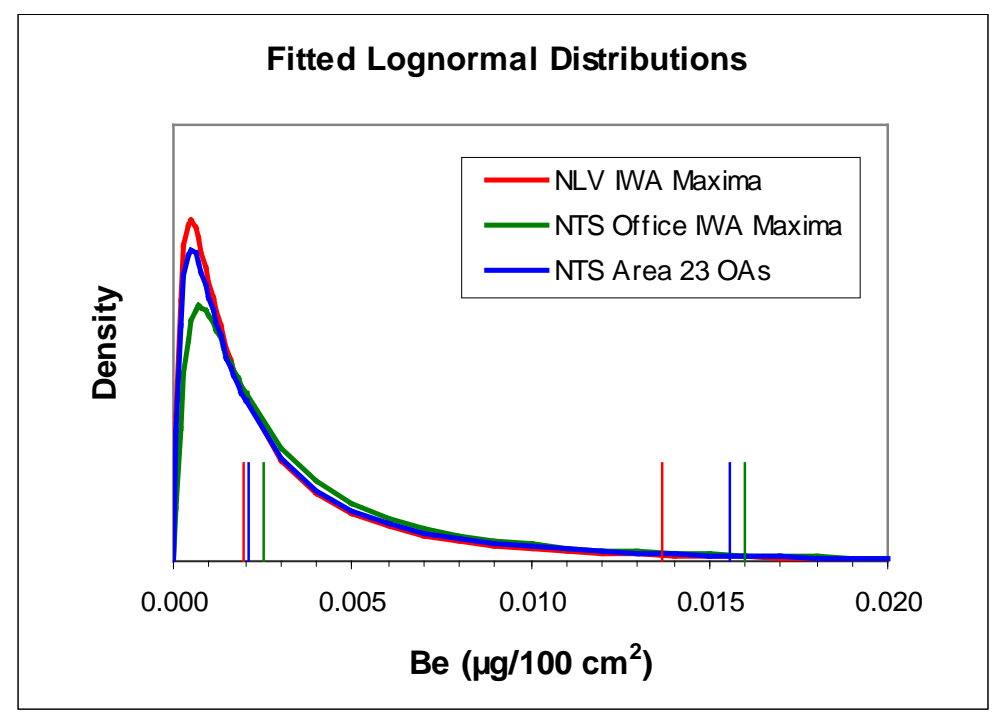

Figure 4: Comparisons of the fitted lognormal distributions in Figures 1, 2, and 3. Vertical lines mark the medians and 95th percentiles.

lognormal distributions fit quite well; “detects-only” probability plot correlations are 0.945 and 0.987, respectively, for the data at and above the cutoff. Figure 3 shows the pooled distribution of most of the Be measurements obtained in Areas in NTS Area 23 facilities; four facilities with at least one wipe measurement each above the RC are omitted. The probability plot correlation is 
0.997 for values above the cutoff. Figure 4 compares the fitted distributions for Figures 1 through 3. The short vertical lines are at the median and $95^{\text {th }}$ percentiles of the fitted distributions.

Only rarely is this sort of complete information available when carrying out the DQO planning process at the onset of an investigation. When planning the surveys of the suspect buildings in $\mathrm{NLV}$, there was some prior information available, and the results from surveys were then available in planning the rest of the surveys. It is gratifying that the assumed planning distribution (the red probability density function in Figure 4) turned out to be quite similar to the distributions found in the subsequent studies for many facilities. The medians and $95^{\text {th }}$ percentiles are slightly higher in the NTS fitted distributions than in the NLV fitted distribution. We note that the NTS Area 23 Area distribution tends slightly toward higher values in spite of being a distribution for all measurements rather than a distribution for IWA maxima; this is consistent with sampling surfaces in inherently dustier warehouse and shop areas.

For planning purposes in this document, we will assume a lognormal distribution with parameters $\mu=-6.25$ and $\sigma=1.19$; these are approximately the mean and standard deviation of the fitted distribution of NLV IWA log maxima. For individual facilities, the actual means and standard deviations will vary. Standard deviations for individual facilities should typically be smaller, since the fitted distributions shown in Figures 1 through 4 are each pooled across several facilities.

Appendix A of "Planning a Beryllium Characterization Sampling Campaign for a DOE Facility" (2005) discusses the UTL approach in greater detail, providing the basic formula used, a table of the multipliers $\mathrm{K}$ needed, and graphs giving the minimum number of wipe samples desired at a facility as a function of the $Z_{R C}$ value, which is the distance between the RC and the data mean, in standardized units on the log scale. Details of the sample size computation are given by Davis (2004).

In sampling Area facilities, the assumption of homogeneity would in principle justify using the same number of samples in a small laboratory as in a mammoth warehouse. One must be sensitive to the possible negative stakeholder reaction to doing so and, in fact, there are always real concerns that some areas of a facility might somehow be more likely to be contaminated than others. Hence, in sampling Areas, we also require an approximate minimum grid spacing, which is on the order of $20 \mathrm{ft}$. (A facility so small that at most eight samples are obtained is treated as a single IWA.) Furthermore, in nearly every case we conducted a walkthrough of the facility before deciding on the sampling plan. In some cases, a greater overall sampling intensity or preferential attention to certain areas was suggested by observations during the walkthrough, such as with areas in which metal machining operations had taken place or which were particularly dusty.

Similar computations can be used for determining sample sizes when using the PL approach with IWA maxima. In this case, however, the desirable minimum sample size will depend on the total number of IWAs as well as $Z_{R C}$. Appendix B of "Planning a Beryllium Characterization Sampling Campaign for a DOE Facility” describes the procedure in greater detail, provides tables 
of the multipliers $\mathrm{K}$ needed, and gives graphs for determining the minimum numbers of observations again as functions of $Z_{R C}$. For example, with 100 IWAs total and $Z_{R C}=3.90$, we should sample $16,31,40$, 48, or 61 IWAs for $50 \%$, $80 \%, 90 \%$, 95\%, or $99 \%$ power, respectively. Recall that sampling one IWA actually involves four or more samples, according to the sampling protocol described above, so these sample sizes cannot be compared directly with those obtained from the UTL approach.

[Technical notes: As with the UTL approach, if it appears that the desired sample size should be greater than 59, one could revert to a procedure that is a mixture of the UTL and PL approaches described here, which would be to use the UTL approach with the IWA maxima as data. In that case, one would not sample more than 59 IWAs in any facility. Also, in some situations it may appear that the Appendix B graphs require that all IWAs be sampled. This occurs when $Z_{R C}$ is so small that one simply cannot obtain the desired power otherwise. In this and all other situations, we implicitly ignore the sampling variability within IWAs, and assume that any IWA whose maximum wipe value is not greater than RC should be considered clean.]

\section{$\underline{\text { On Data Distributions and Transformations }}$}

The planning data and calculations presented above suggest that actual data should be expected to follow a lognormal distribution; that is, that the actual measurements should be transformed by taking logs, and the UTL or PL comparisons with the RC should then be performed on the transformed scale. In fact, if a facility is particularly homogeneous, it may be that the distribution of actual measurements is adequately normal (i.e., Gaussian or "bell-shaped-curve”) to carry out the computations reasonably reliable without data transformation. This can be checked for larger facilities directly. If this is the case, it is likely that the sample size determinations using the log data distributions described above will be conservative. Of course, that will not be known until the data become available, but should be taken into account in preparing the UTLs or PLalls. [Note: In implementation, the possibly conservative lognormal approach was used in all cases; see Appendix 3.]

\section{Statistical Analysis of the Resulting Data}

\section{The UTL Approach}

A $100 \gamma \%-100 \beta \%$ UTL is a $100 \gamma \%$ UTL for the $100 \beta^{\text {th }}$ percentile of a distribution. $\beta$ is called the content and $\gamma$ the confidence. It is traditional in IH applications to use $\beta=\gamma=95 \%$; we use a higher value of $\gamma(99 \%)$ with a facility containing both Areas being handled via the UTL approach and IWAs being handled via the PLall approach, as described previously.

There are two common types of UTLs. Nonparametric UTLs are computed as an appropriate upper ordered value (always the maximum value in this setting) of the data; in order to achieve the target $\beta=\gamma=95 \%$ one must have at least 59 observations, and there is rarely incentive to obtain more. The UTL is then the largest observation, and the decision about whether the facility passes or not is based on whether the UTL is at most the RC or not. Although in principle one might obtain a larger number of observations and mathematically set the UTL to the second or third, etc., highest value, the additional requirement that all measurements should not be greater than the RC would render doing so futile. 
The other type of UTL is based on normal distribution theory. Here UTL $=\bar{X}+\mathrm{K} S$, where $\bar{X}$ and $S$ are the usual sample mean and standard deviation on a suitably transformed scale, and the multiplier K is obtained as described elsewhere. One uses this UTL in the same manner as the nonparametric UTL in making the decision. Tables of $\mathrm{K}$ as a function of the number of observations are given below for 95\%-95\% UTLs; algorithms have been implemented to compute $\mathrm{K}$ for arbitrary $\beta$ and $\gamma$.

\begin{tabular}{cc|cc|cc|cc}
\hline \hline \multicolumn{8}{c}{ K values for 95\%-95\% UTLs } \\
$\mathbf{n}$ & $\mathbf{K}$ & $\mathbf{N}$ & $\mathbf{K}$ & $\mathbf{n}$ & $\mathbf{K}$ & $\mathbf{n}$ & $\mathbf{K}$ \\
\hline & & 16 & 2.5237 & 31 & 2.2080 & 46 & 2.0865 \\
2 & 26.2597 & 17 & 2.4863 & 32 & 2.1968 & 47 & 2.0808 \\
3 & 7.6559 & 18 & 2.4529 & 33 & 2.1863 & 48 & 2.0754 \\
4 & 5.1439 & 19 & 2.4230 & 34 & 2.1762 & 49 & 2.0701 \\
5 & 4.2027 & 20 & 2.3960 & 35 & 2.1667 & 50 & 2.0650 \\
6 & 3.7077 & 21 & 2.3714 & 36 & 2.1577 & 51 & 2.0601 \\
7 & 3.3995 & 22 & 2.3490 & 37 & 2.1491 & 52 & 2.0553 \\
8 & 3.1873 & 23 & 2.3283 & 38 & 2.1408 & 53 & 2.0507 \\
9 & 3.0312 & 24 & 2.3093 & 39 & 2.1330 & 54 & 2.0463 \\
10 & 2.9110 & 25 & 2.2917 & 40 & 2.1255 & 55 & 2.0419 \\
11 & 2.8150 & 26 & 2.2753 & 41 & 2.1183 & 56 & 2.0377 \\
12 & 2.7363 & 27 & 2.2600 & 42 & 2.1114 & 57 & 2.0337 \\
13 & 2.6705 & 28 & 2.2458 & 43 & 2.1048 & 58 & 2.0297 \\
14 & 2.6144 & 29 & 2.2324 & 44 & 2.0985 & 59 & 2.0259 \\
15 & 2.5660 & 30 & 2.2198 & 45 & 2.0924 & 60 & 2.0222 \\
\hline 62 & 2.0150 & 72 & 1.9843 & 82 & 1.9600 & 92 & 1.9401 \\
64 & 2.0082 & 74 & 1.9790 & 84 & 1.9557 & 94 & 1.9365 \\
66 & 2.0018 & 76 & 1.9739 & 86 & 1.9516 & 96 & 1.9331 \\
68 & 1.9957 & 78 & 1.9691 & 88 & 1.9476 & 98 & 1.9298 \\
70 & 1.9899 & 80 & 1.9644 & 90 & 1.9438 & 100 & 1.9265 \\
\hline 105 & 1.9189 & 130 & 1.8883 & 155 & 1.8658 & 180 & 1.8485 \\
110 & 1.9119 & 135 & 1.8833 & 160 & 1.8620 & 185 & 1.8455 \\
115 & 1.9054 & 140 & 1.8785 & 165 & 1.8584 & 190 & 1.8427 \\
120 & 1.8993 & 145 & 1.8741 & 170 & 1.8550 & 195 & 1.8399 \\
125 & 1.8936 & 150 & 1.8698 & 175 & 1.8517 & 200 & 1.8372 \\
\hline 300 & 1.7996 & 400 & 1.7778 & 500 & 1.7630 & 1000 & 1.7273 \\
\hline \hline
\end{tabular}




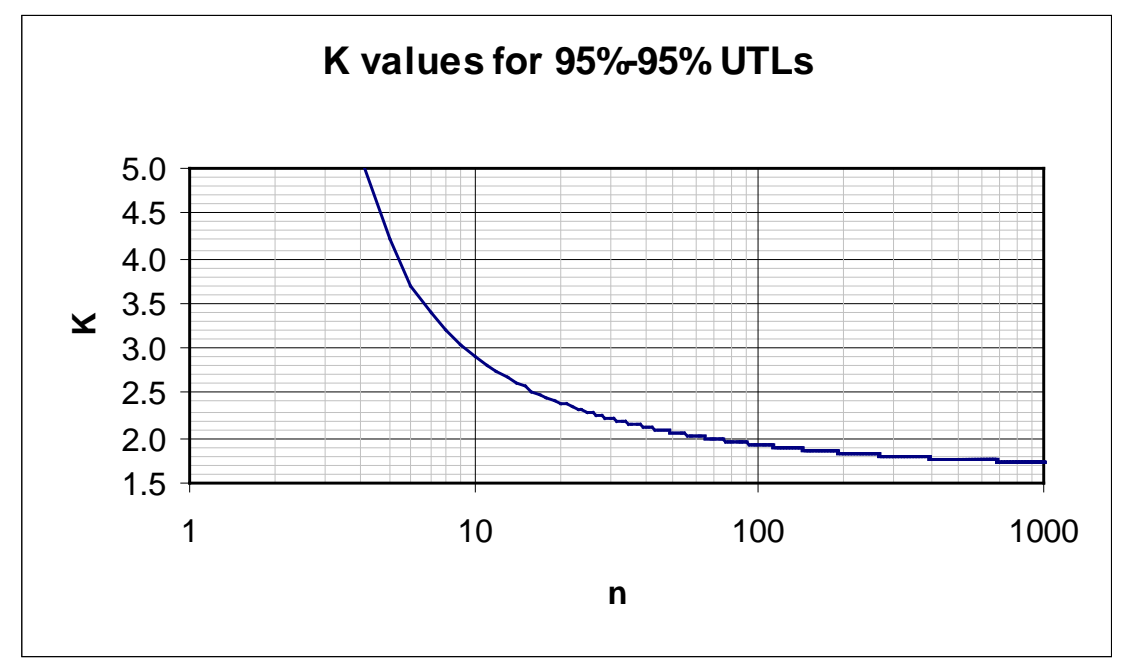

[Note: As described in Appendix 3, the methodology ultimately selected for handling the ubiquitous negative values was to add a small constant delta to all values, take logs of the $(x+$ delta), use the normal-theory UTL computations with the results, exponentiate the UTL, and then subtract delta. This methodology, in particular the selection of delta for various laboratories and worker environments, is described in detail in Appendix 3; see also Davis, Field, and Gran (2009).]

\section{The PL Approach}

The PL approach is considerably different from the UTL approach conceptually. The idea is that we have observations from some $n$ items from a population, and from these we would like to make a prediction limit that should include all of the next $r$ items with suitably high confidence. In this setting, $r=\mathrm{N}-n$, where $\mathrm{N}$ is the total number of IWAs in the facility to be surveyed and $r$ is the number of unsampled IWAs.

More explicitly, our samplers were instructed to sample locations within an IWA thought most likely to produce elevated Be values. Any sampled IWA whose largest measurement is not greater than the RC is found to be clean. In our sample of $n$, IWAs all have been found clean, so we attempt to determine based on these data that, with reasonably high confidence, all the remaining IWAs would similarly be found clean if they were sampled as well. The mechanism for doing so is PLall, a PL for the largest value to be found in the remaining unsampled IWAs.

A nonparametric PLall approach would require prohibitively large numbers of observations. The normal distribution theory computation of PLall uses the algorithm described by Davis and McNichols (1987). The present computation makes prediction limits for " 1 of 1 " future observation at each of $r$ locations, in the terminology of the title of the article.

As with the UTL approach, we compare $\mathrm{PL}=\bar{X}+\mathrm{K} S$, computed on an appropriate scale, with the RC, expressed on the same scale. Unlike the UTL approach, here the sample statistics are computed from the IWA maxima rather than from all the data. The multiplier $\mathrm{K}$ depends on both the sample size $n$ and the number of unsampled IWAs $r$; equivalently, on $n$ and the total number 
of IWAs, denoted $\mathrm{N}$, since $r=\mathrm{N}-n$. This makes tables of $\mathrm{K}$ as a function of $n$ rather cumbersome; tables are given here for ten selected values of N. Software has been developed and can be made available for computing $\mathrm{K}$ for arbitrary $n$, confidence level (95\% here), $r$, and degrees of freedom (equal to $n-1$ here). The one graph presented here shows K plotted against the proportion of IWAs sampled (i.e., $n$ / N) for each value of $\mathrm{N}$; it shows some regularity across $\mathrm{N}$ except for the highest and lowest values of $n$ allowable in each case.

\begin{tabular}{cccccc|ccc}
\hline \hline & \multicolumn{7}{c}{ K values for 95\% PLall } \\
$\mathrm{N}=$ & 5 & 10 & 15 & 20 & 30 & $\mathrm{~N}=$ & 20 & 30 \\
$\mathrm{n}$ & $\mathrm{K}$ & $\mathrm{K}$ & $\mathrm{K}$ & $\mathrm{K}$ & $\mathrm{K}$ & $\mathrm{n}$ & $\mathrm{K}$ & $\mathrm{K}$ \\
\hline 3 & 4.5722 & 6.9455 & 7.9529 & 8.5824 & 9.3875 & 17 & 2.3730 & 3.0757 \\
4 & 2.6312 & 4.6866 & 5.3915 & 5.8160 & 6.3492 & 18 & 2.1534 & 3.0137 \\
5 & & 3.7880 & 4.4179 & 4.7783 & 5.2200 & 19 & 1.7791 & 2.9524 \\
6 & & 3.2587 & 3.8906 & 4.2279 & 4.6290 & 20 & & 2.8906 \\
7 & & 2.8569 & 3.5452 & 3.8788 & 4.2611 & 21 & & 2.8272 \\
8 & 2.4711 & 3.2883 & 3.6310 & 4.0069 & 22 & & 2.7606 \\
9 & & 1.9601 & 3.0769 & 3.4404 & 3.8179 & 23 & & 2.6890 \\
10 & & & 2.8868 & 3.2841 & 3.6699 & 24 & & 2.6098 \\
11 & & & 2.6998 & 3.1490 & 3.5491 & 25 & & 2.5193 \\
12 & & 2.4961 & 3.0262 & 3.4470 & 26 & & 2.4111 \\
13 & & 2.2416 & 2.9095 & 3.3583 & 27 & & 2.2732 \\
14 & & 1.8331 & 2.7932 & 3.2792 & 28 & & 2.0778 \\
15 & & & 2.6713 & 3.2070 & 29 & & 1.7303 \\
16 & & & 2.5357 & 3.1397 & & & \\
\hline \hline
\end{tabular}




\begin{tabular}{|c|c|c|c|c|c|c|c|c|c|c|c|c|}
\hline \multicolumn{13}{|c|}{ K values for 95\% PLall } \\
\hline $\begin{array}{l}\mathrm{N}= \\
\mathrm{n}\end{array}$ & $\begin{array}{l}50 \\
\mathrm{~K}\end{array}$ & $\begin{array}{l}75 \\
\mathrm{~K}\end{array}$ & $\begin{array}{c}100 \\
\mathrm{~K}\end{array}$ & $\begin{array}{l}\mathrm{N}= \\
\mathrm{n}\end{array}$ & $\begin{array}{l}50 \\
\mathrm{~K}\end{array}$ & $\begin{array}{c}75 \\
\mathrm{~K} \\
\end{array}$ & $\begin{array}{c}100 \\
\mathrm{~K}\end{array}$ & $\begin{array}{l}\mathrm{N}= \\
\mathrm{n}\end{array}$ & $\begin{array}{l}75 \\
\mathrm{~K} \\
\end{array}$ & $\begin{array}{c}100 \\
\mathrm{~K}\end{array}$ & $\begin{array}{l}\mathrm{N}= \\
\mathrm{n}\end{array}$ & $\begin{array}{c}100 \\
\mathrm{~K}\end{array}$ \\
\hline & & & & 26 & 3.1698 & 3.4492 & 3.6056 & 51 & 3.0116 & 3.2613 & 76 & 2.9597 \\
\hline & & & & 27 & 3.1406 & 3.4267 & 3.5846 & 52 & 2.9933 & 3.2506 & 77 & 2.9437 \\
\hline & & & & 28 & 3.1118 & 3.4054 & 3.5649 & 53 & 2.9744 & 3.2399 & 78 & 2.9270 \\
\hline 4 & 6.9489 & 7.3858 & 7.6798 & 29 & 3.0831 & 3.3849 & 3.5463 & 54 & 2.9550 & 3.2292 & 79 & 2.9097 \\
\hline 5 & 5.7086 & 6.0615 & 6.2984 & 30 & 3.0546 & 3.3652 & 3.5285 & 55 & 2.9348 & 3.2186 & 80 & 2.8915 \\
\hline 6 & 5.0638 & 5.3747 & 5.5825 & 31 & 3.0258 & 3.3462 & 3.5116 & 56 & 2.9138 & 3.2079 & 81 & 2.8724 \\
\hline 7 & 4.6661 & 4.9523 & 5.1426 & 32 & 2.9967 & 3.3278 & 3.4955 & 57 & 2.8919 & 3.1971 & 82 & 2.8524 \\
\hline 8 & 4.3943 & 4.6646 & 4.8435 & 33 & 2.9671 & 3.3100 & 3.4800 & 58 & 2.8690 & 3.1863 & 83 & 2.8313 \\
\hline 9 & 4.1954 & 4.4550 & 4.6259 & 34 & 2.9366 & 3.2925 & 3.4651 & 59 & 2.8449 & 3.1754 & 84 & 2.8089 \\
\hline 10 & 4.0424 & 4.2948 & 4.4599 & 35 & 2.9052 & 3.2754 & 3.4507 & 60 & 2.8194 & 3.1644 & 85 & 2.7850 \\
\hline 11 & 3.9204 & 4.1678 & 4.3285 & 36 & 2.8725 & 3.2587 & 3.4368 & 61 & 2.7922 & 3.1533 & 86 & 2.7596 \\
\hline 12 & 3.8202 & 4.0642 & 4.2217 & 37 & 2.8383 & 3.2422 & 3.4234 & 62 & 2.7632 & 3.1421 & 87 & 2.7321 \\
\hline 13 & 3.7357 & 3.9778 & 4.1329 & 38 & 2.8021 & 3.2259 & 3.4103 & 63 & 2.7319 & 3.1307 & 88 & 2.7025 \\
\hline 14 & 3.6632 & 3.9043 & 4.0577 & 39 & 2.7634 & 3.2097 & 3.3975 & 64 & 2.6980 & 3.1192 & 89 & 2.6701 \\
\hline 15 & 3.5999 & 3.8409 & 3.9930 & 40 & 2.7217 & 3.1937 & 3.3852 & 65 & 2.6609 & 3.1075 & 90 & 2.6345 \\
\hline 16 & 3.5438 & 3.7854 & 3.9366 & 41 & 2.6762 & 3.1777 & 3.3731 & 66 & 2.6198 & 3.0956 & 91 & 2.5950 \\
\hline 17 & 3.4933 & 3.7363 & 3.8870 & 42 & 2.6259 & 3.1617 & 3.3612 & 67 & 2.5737 & 3.0835 & 92 & 2.5504 \\
\hline 18 & 3.4474 & 3.6924 & 3.8428 & 43 & 2.5691 & 3.1457 & 3.3495 & 68 & 2.5212 & 3.0711 & 93 & 2.4995 \\
\hline 19 & 3.4053 & 3.6527 & 3.8032 & 44 & 2.5038 & 3.1297 & 3.3380 & 69 & 2.4601 & 3.0584 & 94 & 2.4400 \\
\hline 20 & 3.3662 & 3.6165 & 3.7673 & 45 & 2.4264 & 3.1135 & 3.3267 & 70 & 2.3870 & 3.0455 & 95 & 2.3687 \\
\hline 21 & 3.3295 & 3.5834 & 3.7347 & 46 & 2.3310 & 3.0972 & 3.3156 & 71 & 2.2960 & 3.0322 & 96 & 2.2796 \\
\hline 22 & 3.2949 & 3.5528 & 3.7047 & 47 & 2.2060 & 3.0807 & 3.3045 & 72 & 2.1759 & 3.0186 & 97 & 2.1615 \\
\hline 23 & 3.2619 & 3.5243 & 3.6772 & 48 & 2.0246 & 3.0639 & 3.2936 & 73 & 2.0000 & 3.0046 & 98 & 1.9882 \\
\hline 24 & 3.2302 & 3.4978 & 3.6516 & 49 & 1.6942 & 3.0468 & 3.2828 & 74 & 1.6772 & 2.9901 & 99 & 1.6689 \\
\hline 25 & 3.1996 & 3.4728 & 3.6278 & 50 & & 3.0294 & 3.2720 & 75 & & 2.9752 & & \\
\hline
\end{tabular}




\begin{tabular}{cc|cc|cc|cc|cc|cc}
\hline \hline & & \multicolumn{10}{c}{ K values for 95\% PLall } \\
$\mathrm{N}=$ & 150 & $\mathrm{~N}=$ & 150 & $\mathrm{~N}=$ & 150 & $\mathrm{~N}=$ & 150 & $\mathrm{~N}=$ & 150 & $\mathrm{~N}=$ & 150 \\
$\mathrm{n}$ & $\mathrm{K}$ & $\mathrm{n}$ & $\mathrm{K}$ & $\mathrm{n}$ & $\mathrm{K}$ & $\mathrm{n}$ & $\mathrm{K}$ & $\mathrm{n}$ & $\mathrm{K}$ & $\mathrm{n}$ & $\mathrm{K}$ \\
\hline & & 26 & 3.7966 & 51 & 3.4966 & 76 & 3.3325 & 101 & 3.1685 & 126 & 2.9186 \\
& & 27 & 3.7763 & 52 & 3.4891 & 77 & 3.3264 & 102 & 3.1610 & 127 & 2.9039 \\
& & 28 & 3.7572 & 53 & 3.4816 & 78 & 3.3202 & 103 & 3.1534 & 128 & 2.8885 \\
4 & 8.0761 & 29 & 3.7393 & 54 & 3.4743 & 79 & 3.3141 & 104 & 3.1456 & 129 & 2.8724 \\
5 & 6.6171 & 30 & 3.7225 & 55 & 3.4672 & 80 & 3.3080 & 105 & 3.1377 & 130 & 2.8555 \\
6 & 5.8615 & 31 & 3.7066 & 56 & 3.4601 & 81 & 3.3018 & 106 & 3.1297 & 131 & 2.8377 \\
7 & 5.3976 & 32 & 3.6916 & 57 & 3.4532 & 82 & 3.2956 & 107 & 3.1215 & 132 & 2.8189 \\
8 & 5.0824 & 33 & 3.6773 & 58 & 3.4463 & 83 & 3.2894 & 108 & 3.1131 & 133 & 2.7990 \\
9 & 4.8533 & 34 & 3.6637 & 59 & 3.4396 & 84 & 3.2831 & 109 & 3.1046 & 134 & 2.7777 \\
10 & 4.6787 & 35 & 3.6507 & 60 & 3.4329 & 85 & 3.2769 & 110 & 3.0959 & 135 & 2.7551 \\
11 & 4.5409 & 36 & 3.6383 & 61 & 3.4263 & 86 & 3.2706 & 111 & 3.0869 & 136 & 2.7307 \\
12 & 4.4290 & 37 & 3.6265 & 62 & 3.4197 & 87 & 3.2642 & 112 & 3.0778 & 137 & 2.7045 \\
13 & 4.3360 & 38 & 3.6151 & 63 & 3.4133 & 88 & 3.2578 & 113 & 3.0685 & 138 & 2.6760 \\
14 & 4.2577 & 39 & 3.6041 & 64 & 3.4069 & 89 & 3.2514 & 114 & 3.0589 & 139 & 2.6448 \\
15 & 4.1904 & 40 & 3.5936 & 65 & 3.4005 & 90 & 3.2449 & 115 & 3.0491 & 140 & 2.6104 \\
16 & 4.1321 & 41 & 3.5834 & 66 & 3.3942 & 91 & 3.2383 & 116 & 3.0390 & 141 & 2.5721 \\
17 & 4.0808 & 42 & 3.5735 & 67 & 3.3879 & 92 & 3.2317 & 117 & 3.0287 & 142 & 2.5288 \\
18 & 4.0354 & 43 & 3.5640 & 68 & 3.3817 & 93 & 3.2250 & 118 & 3.0180 & 143 & 2.4792 \\
19 & 3.9948 & 44 & 3.5547 & 69 & 3.3754 & 94 & 3.2182 & 119 & 3.0070 & 144 & 2.4211 \\
20 & 3.9583 & 45 & 3.5458 & 70 & 3.3693 & 95 & 3.2114 & 120 & 2.9957 & 145 & 2.3512 \\
21 & 3.9252 & 46 & 3.5371 & 71 & 3.3631 & 96 & 3.2045 & 121 & 2.9839 & 146 & 2.2638 \\
22 & 3.8951 & 47 & 3.5286 & 72 & 3.3570 & 97 & 3.1975 & 122 & 2.9718 & 147 & 2.1477 \\
23 & 3.8674 & 48 & 3.5203 & 73 & 3.3508 & 98 & 3.1904 & 123 & 2.9593 & 148 & 1.9767 \\
24 & 3.8420 & 49 & 3.5123 & 74 & 3.3447 & 99 & 3.1832 & 124 & 2.9462 & 149 & 1.6607 \\
25 & 3.8185 & 50 & 3.5044 & 75 & 3.3386 & 100 & 3.1759 & 125 & 2.9327 & 150 & \\
\hline \hline
\end{tabular}

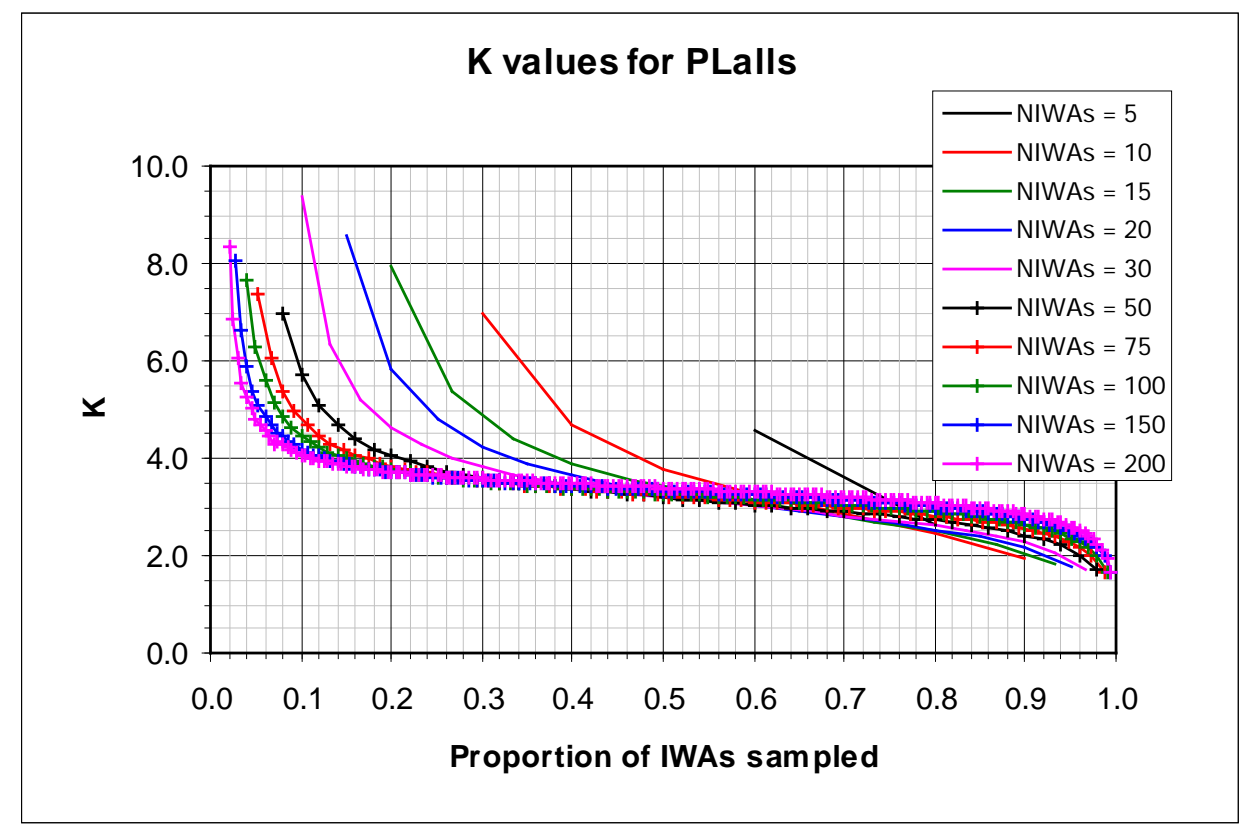

Worker Environment Beryllium Characterization Study 


\begin{tabular}{|c|c|c|c|c|c|c|c|c|c|c|c|}
\hline \multicolumn{12}{|c|}{$\mathrm{K}$ values for $95 \%$ PLall } \\
\hline $\begin{array}{l}\mathrm{N}= \\
\mathrm{n}\end{array}$ & $\begin{array}{c}200 \\
K\end{array}$ & $\begin{array}{l}\mathrm{N}= \\
\mathrm{n}\end{array}$ & $\begin{array}{c}200 \\
\mathrm{~K}\end{array}$ & $\begin{array}{l}\mathrm{N}= \\
\mathrm{n}\end{array}$ & $\begin{array}{c}200 \\
\text { K }\end{array}$ & $\begin{array}{l}\mathrm{N}= \\
\mathrm{n}\end{array}$ & $\begin{array}{c}200 \\
\mathrm{~K}\end{array}$ & $\begin{array}{l}\mathrm{N}= \\
\mathrm{n}\end{array}$ & $\begin{array}{c}200 \\
\mathrm{~K}\end{array}$ & $\begin{array}{l}\mathrm{N}= \\
\mathrm{n}\end{array}$ & $\begin{array}{c}200 \\
\mathrm{~K}\end{array}$ \\
\hline 4 & 8.3464 & 37 & 3.7506 & 70 & 3.5222 & 103 & 3.3800 & 136 & 3.2278 & 169 & 2.9863 \\
\hline 5 & 6.8343 & 38 & 3.7396 & 71 & 3.5175 & 104 & 3.3758 & 137 & 3.2224 & 170 & 2.9754 \\
\hline 6 & 6.0514 & 39 & 3.7291 & 72 & 3.5128 & 105 & 3.3716 & 138 & 3.2169 & 171 & 2.9642 \\
\hline 7 & 5.5708 & 40 & 3.7190 & 73 & 3.5081 & 106 & 3.3674 & 139 & 3.2114 & 172 & 2.9525 \\
\hline 8 & 5.2444 & 41 & 3.7093 & 74 & 3.5035 & 107 & 3.3632 & 140 & 3.2058 & 173 & 2.9404 \\
\hline 9 & 5.0072 & 42 & 3.7000 & 75 & 3.4989 & 108 & 3.3589 & 141 & 3.2001 & 174 & 2.9278 \\
\hline 10 & 4.8265 & 43 & 3.6910 & 76 & 3.4944 & 109 & 3.3547 & 142 & 3.1943 & 175 & 2.9147 \\
\hline 11 & 4.6840 & 44 & 3.6824 & 77 & 3.4899 & 110 & 3.3504 & 143 & 3.1884 & 176 & 2.9011 \\
\hline 12 & 4.5683 & 45 & 3.6740 & 78 & 3.4855 & 111 & 3.3461 & 144 & 3.1825 & 177 & 2.8868 \\
\hline 13 & 4.4723 & 46 & 3.6660 & 79 & 3.4810 & 112 & 3.3418 & 145 & 3.1764 & 178 & 2.8719 \\
\hline 14 & 4.2914 & 47 & 3.6582 & 80 & 3.4766 & 113 & 3.3375 & 146 & 3.1703 & 179 & 2.8562 \\
\hline 15 & 4.3220 & 48 & 3.6505 & 81 & 3.4723 & 114 & 3.3331 & 147 & 3.1640 & 180 & 2.8397 \\
\hline 16 & 4.2619 & 49 & 3.6432 & 82 & 3.4680 & 115 & 3.3287 & 148 & 3.1576 & 181 & 2.8224 \\
\hline 17 & 4.2092 & 50 & 3.6361 & 83 & 3.4637 & 116 & 3.3243 & 149 & 3.1512 & 182 & 2.8040 \\
\hline 18 & 4.1626 & 51 & 3.6292 & 84 & 3.4594 & 117 & 3.3199 & 150 & 3.1446 & 183 & 2.7845 \\
\hline 19 & 4.1210 & 52 & 3.6224 & 85 & 3.4551 & 118 & 3.3154 & 151 & 3.1378 & 184 & 2.7637 \\
\hline 20 & 4.0836 & 53 & 3.6158 & 86 & 3.4509 & 119 & 3.3109 & 152 & 3.1310 & 185 & 2.7415 \\
\hline 21 & 4.0498 & 54 & 3.6094 & 87 & 3.4467 & 120 & 3.3063 & 153 & 3.1240 & 186 & 2.7176 \\
\hline 22 & 4.0190 & 55 & 3.6031 & 88 & 3.4424 & 121 & 3.3018 & 154 & 3.1168 & 187 & 2.6918 \\
\hline 23 & 3.9909 & 56 & 3.5970 & 89 & 3.4382 & 122 & 3.2972 & 155 & 3.1096 & 188 & 2.6637 \\
\hline 24 & 3.9651 & 57 & 3.5910 & 90 & 3.4341 & 123 & 3.2925 & 156 & 3.1021 & 189 & 2.6330 \\
\hline 25 & 3.9413 & 58 & 3.5851 & 91 & 3.4299 & 124 & 3.2878 & 157 & 3.0945 & 190 & 2.5991 \\
\hline 26 & 3.9193 & 59 & 3.5794 & 92 & 3.4257 & 125 & 3.2831 & 158 & 3.0867 & 191 & 2.5613 \\
\hline 27 & 3.8988 & 60 & 3.5737 & 93 & 3.4216 & 126 & 3.2783 & 159 & 3.0788 & 192 & 2.5186 \\
\hline 28 & 3.8796 & 61 & 3.5682 & 94 & 3.4174 & 127 & 3.2735 & 160 & 3.0706 & 193 & 2.4695 \\
\hline 29 & 3.8618 & 62 & 3.5628 & 95 & 3.4133 & 128 & 3.2687 & 161 & 3.0622 & 194 & 2.4120 \\
\hline 30 & 3.8450 & 63 & 3.5574 & 96 & 3.4091 & 129 & 3.2637 & 162 & 3.0536 & 195 & 2.3428 \\
\hline 31 & 3.8292 & 64 & 3.5522 & 97 & 3.4050 & 130 & 3.2588 & 163 & 3.0448 & 196 & 2.2561 \\
\hline 32 & 3.8143 & 65 & 3.5470 & 98 & 3.4008 & 131 & 3.2538 & 164 & 3.0358 & 197 & 2.1409 \\
\hline 33 & 3.8002 & 66 & 3.5419 & 99 & 3.3966 & 132 & 3.2487 & 165 & 3.0265 & 198 & 1.9711 \\
\hline 34 & 3.7869 & 67 & 3.5369 & 100 & 3.3925 & 133 & 3.2436 & 166 & 3.0169 & 199 & 1.6567 \\
\hline 35 & 3.7742 & 68 & 3.5319 & 101 & 3.3883 & 134 & 3.2384 & 167 & 3.0070 & & \\
\hline 36 & 3.7621 & 69 & 3.5271 & 102 & 3.3842 & 135 & 3.2331 & 168 & 2.9968 & & \\
\hline
\end{tabular}

The UTL_cluster sampling approach [Added December 2007]

As noted previously, both the PLall and UTL approaches when used with IWA maxima are more stringent than the approach contemplated in the draft DOE Technical Standard, since the latter is based on UTLs for the distributions of all measurements (not IWA maxima) obtained from random (not biased) sampling at a facility. It turns out that these approaches are problematic with certain facilities, notably those with few IWAs and a few atypically high values in some IWAs. Some of those atypical values come from locations that might arguably not be considered part of the normal worker environment; some come from a suspect chemical analysis (see Appendix 3); some have both factors involved.

Hence there is motivation to develop a UTL approach that uses all of the data obtained from IWA facilities. That is presented in Appendix 4. The statistical model is that of traditional cluster sampling, in which (for cost and 
convenience) a sampling effort first identifies clusters within a population and then randomly samples within clusters. The clusters correspond to IWAs here. The mathematical setup for UTLs with cluster sampling is nonstandard, in that the usual cluster sampling efforts aim at estimating population means, whereas the $95^{\text {th }}$ percentile is the target here; the details are provided in Appendix 4. The statistical issue is that there may be a systematic, though random, cluster-to-cluster (here, IWA-to-IWA) variation that would make the measurements within an IWA correlated, so that the usual UTL computation would assume that more statistically independent information is available than we have.

UTL_cluster was computed only for those facilities fitting the above description; that is, both UTL and PLall based on IWA maxima are higher than the RC although all observations are at most equal to the RC. The UTL_cluster analysis is still more conservative than the UTL approach based on simple random sampling discussed in the current draft DOE Technical Standard, in that the samplers were still looking for sampling locations particularly likely to provide high Be values rather than random locations within the facility. Hence, a facility is taken as "passing" if any of PLall, UTL, or UTL_cluster is not greater than the RC. 


\section{Appendix 2 STATISTICAL ANALYSIS DETAILS}

\section{Introduction}

This appendix contains three items:

- A brief review of the statistical methodology employed,

- A concise summary table of the results by facility, and

- A detailed listing of the results for each facility.

\section{Statistical Methodology}

As discussed in Appendix 1, the statistical methodology depends on the nature of the facility in question. There are four possibilities:

- A facility consists of multiple Individual Work Areas (IWAs);

- A facility consists of one or more open Areas, such as a warehouse or shop;

- A facility has portions consisting of one or more IWAs along with open areas (Areas); and

- A facility consists of just one or a few IWAs, all of which have been sampled.

In the first case (multiple IWAs only), a random selection of IWAs was sampled. The IWA value is the largest measurement obtained in that IWA, the IWA maximum. Two sample statistics are computed initially. The first is PLall, a prediction limit designed to contain all the IWA maxima for the remaining unsampled IWAs with $95 \%$ confidence. The second is the upper tolerance limit (UTL), designed to be at least as large as the $95^{\text {th }}$ percentile of the distribution of the IWA maxima, which in turn will be at least as large as the $95^{\text {th }}$ percentile of the distribution of all possible measurements, with 95\% confidence. This is compared with the U.S. Department of Energy (DOE) release criterion (RC) of 0.2 micrograms per wipe ( $\mu \mathrm{g} /$ wipe), with a wipe having covered an area of 100 square centimeters $\left(\mathrm{cm}^{2}\right)$. If at least one of these is no greater than the RC (and no individual measurement exceeds the RC), the facility passes.

The PLall approach was that originally proposed in discussions with the U.S. Department of Energy, National Nuclear Security Administration Headquarters (NNSA/HQ) incident investigation team. The UTL approach is more nearly comparable with that favored in the draft DOE Technical Standard under discussion by the Beryllium Health \& Safety Committee (BH\&SC). The latter is a less stringent standard in most cases; the exceptions involve those facilities with only a few unsampled IWAs.

In some situations the UTL approach is still quite stringent since it uses only IWA maxima, which can be atypical observations (“outliers”). A third approach was developed for these situations, which uses all the data from each IWA. This is termed the UTL cluster analysis; see the detailed discussion in Appendix 4. This approach is still more conservative than that in the 
current draft DOE Technical Standard, however, since the sampling team was instructed to sample the dirtiest spots within IWAs rather than attempting to obtain random samples.

In the second case (Areas only), a systematic random sampling plan was followed. An UTL is computed from the data. If this is no greater than the RC (and no individual measurement exceeds the RC), the facility passes.

In the third case (both IWAs and Areas), a PLall and UTL are computed for the IWA portion and a UTL for the Area portion. The confidence levels used are 96\% and 99\%, respectively (keeping the overall significance level at the target $5 \%$ ). If both portions of the facility pass (and no individual measurement exceeds the RC), the facility passes. The individual significance levels (alpha $=4 \%$ and $1 \%$, respectively) are intentionally disproportionate, as the IWA maximum comparison is inherently a more stringent comparison than the Area UTL comparison. In one facility (35-2229), there is one IWA portion and two Area portions of different natures; in that facility three tests were conducted, using significance levels of $3 \%, 1 \%$, and $1 \%$, respectively. Where the IWA cluster analysis is used, in some cases the significance levels are set equal. In any case, the sum of the significance levels is the target $5 \%$.

In the last case, the IWA maximum (maxima) is (are) simply compared with the DOE RC. If no IWA maximum exceeds the DOE RC, the facility passes.

Appendices 1, 3, and 4 provide the technical details of computing the PLall, IWA, and IWA_cluster values.

\section{Exceptions}

At some facilities one or two isolated high measurements prevent the facility from passing using these decision rules. In these situations one or both of the following strategies were used. One was to evaluate the location which produced the high measurement; if that location is not normally "touchable" in routine work activities (and hence less likely to be cleaned as well as other locations), the statistical analysis would be repeated with the outlying observation removed. Alternatively, and particularly if the high observation(s) exceeded the DOE RC, bulk analyses were obtained in the facility and the beryllium $(\mathrm{Be})$ concentrations and metal ratios were obtained for those bulk samples as described in Appendix 4. If the January 2009 sampling produced MS_new observations above the DOE RC, metal ratios were determined for those high wipes as well. If these were generally consistent with values obtained in Nevada Test Site (NTS) soil samples, the facility is considered to pass. A summary table of the bulk sample Be results and the metal ratio results is included at the end of this appendix.

\section{Summary of Results}

Some 228 facilities were evaluated during this survey, with the following outcomes. 


\begin{tabular}{|c|c|c|}
\hline \multicolumn{3}{|c|}{ Count of Results } \\
\hline \multirow[b]{2}{*}{ Result } & \multicolumn{2}{|c|}{ Number of } \\
\hline & Whole facilities & Portions of facilities \\
\hline Pass & 195 & 9 \\
\hline Pass if outlier(s) omitted & 10 & 3 \\
\hline Pass taking bulk Be and/or metal ratios into account & 8 & 9 \\
\hline Fail & 4 & 1 \\
\hline
\end{tabular}

The following table lists the results for all facilities. There are two color-coded blocks; the left one is for the IWA portion of the facility and the right for the Area portion; a blank indicates that a facility did not have one or the other portion. Facility type is shown at the right.

Detailed results by facility follow this table for facilities for which statistical analysis was performed.

\begin{tabular}{|cl|}
\hline Type & Description \\
\hline CO & Communications facility \\
EX & Experimental facility \\
FO & Field Operations facility \\
LA & Laboratory \\
OF & Office facility \\
QU & Quarters, including cafeteria \\
SH & Shop \\
ST & Storage \\
\hline
\end{tabular}

\begin{tabular}{|c|c|c|}
\hline FACILITY & Type & Result \\
\hline 01-179491 & FO & One IWA, max = 0.076 (MS_arch) \\
\hline 01-181769 & OF & PLall = 0.019 \\
\hline 01-201959 & SH & Max=0.249 (MS_new) $>$ DOE RC; other nine $<0.13$; bulk Be and all ratios OK \\
\hline 01-202137 & FO & One IWA, max $=0.110$ (MS_arch) \\
\hline 01-202138 & FO & One IWA, $\max =0.030(\mathrm{DC})$ \\
\hline 01-202333 & FO & One IWA, $\max =0.036$ \\
\hline 01-202479 & ST & 3 of 4 MS_new > DOE RC, max = 1.184; bulk Be and metal ratios OK \\
\hline 01-202521 & FO & One IWA, max = 0.124 (MS_arch); rest $<0.1$ \\
\hline 01-202655 & FO & Area UTL $=0.041$ \\
\hline 01-202656 & FO & One IWA, max $=0.214$ (MS_arch) $>$ DOE RC (windowsill) rest $<$ 0.1; MS_new $=0.008$ \\
\hline 01-202772 & OF & UTL_cluster $=\mathbf{0 . 0 2 0}$ \\
\hline 01-408564 & FO & One IWA, max = 0.175 (MS_arch) after cleaning \\
\hline 01-U1H CO & FO & One IWA, $\max =0.009(\mathrm{DC})$ \\
\hline 01-U1H HHOS & FO & One IWA, max = 0.092 (MS_arch) \\
\hline 01-U1H LR & FO & Area UTL $=0.014$ \\
\hline 01-U1H TLS & FO & One IWA, max = 0.176 (MS_arch); rest $<0.1$ \\
\hline 01-U1H WM & SH & One IWA, max = $0.003\left(B N \_a d j\right)$ \\
\hline 03-3C36 & OF & $\begin{array}{l}\text { Two MS_new > DOE RC; max = 0.522; high values on window ledge and door top; } \\
\text { UTL_cluster = 0.075 if high values excluded; bulk Be and ratios OK; high wipe ratios } \\
\text { OK except for low Ni/Be }\end{array}$ \\
\hline 05-026143 & FO & One IWA, $\max =0.073(\mathrm{DC})$ \\
\hline 05-105 & ST & One IWA, $\max =0.016(\mathrm{DC})$ \\
\hline 05-131045 & FO & One IWA, max $=0.005(\mathrm{DC})$ \\
\hline 05-131047 & FO & One IWA, max = $0.016(\mathrm{DC})$ \\
\hline 05-202177 & SH & $\begin{array}{l}\text { One IWA, max }=0.250(\mathrm{DC})>\text { DOE RC on outlet box, one more }>0.1 \text {; bulk } \\
\text { concentration and metal ratios OK }\end{array}$ \\
\hline 05-202533 & FO & One IWA, $\max =0.099(\mathrm{DC})$ \\
\hline 05-202617 & FO & One IWA, max = 0.195 (MS_arch) on 10/16/03; rest $<0.1$ \\
\hline 05-2247841 & SH & One IWA, max = $0.074(\mathrm{DC})$ \\
\hline 05-24 & ST & $\begin{array}{l}\text { One }>\text { DOE } \mathrm{RC} ; \max =0.215(\mathrm{AT}) ; \mathrm{UTL}=0.410 ; \mathrm{TRU} \text { pad cover; bulk concentrations } \\
\text { and metal ratios OK }\end{array}$ \\
\hline 05-31 & OF & PLall $=0.096$ \\
\hline
\end{tabular}




\begin{tabular}{|c|c|c|}
\hline 05-32 & $\mathbf{E X}$ & Area UTL $=0.036$ \\
\hline 05-652b & FO & Area UTL $=0.031$ \\
\hline $05-7$ & $\mathbf{O F}$ & PLall = 0.019 \\
\hline 05-8 & $\mathbf{E X}$ & PLall = 0.181; $\max =0.014$ \\
\hline $05-83606$ & FO & One IWA, one swipe $=0.057$ (DC) \\
\hline 05-8A & $\mathbf{E X}$ & One IWA, max = 0.005 (DC) \\
\hline $05-9000203$ & SH & One IWA, max = $0.140(D C) ;$ rest $<0.1$ \\
\hline $05-9103$ & SH & One IWA, max = $0.066(\mathrm{DC})$ \\
\hline 05-9105 & SH & One IWA, max = 0.018 (DC) \\
\hline $05-9122$ & SH & One IWA, max = 0.130 $(\mathrm{DC}) ;$ rest $<0.1$ \\
\hline 05-9139 & SH & One IWA, max = 0.110 (DC); rest $<0.1$ \\
\hline $05-9140$ & SH & One IWA, max = 0.100 (DC); rest $<0.1$ \\
\hline $05-925$ & SH & One IWA, $\max =0.200(\mathrm{DC})=$ DOE RC; rest $<0.1$ \\
\hline 05-93 & SH & One IWA, max = 0.110 (DC); rest $<0.1$ \\
\hline 05-938 & SH & One IWA, max = 0.016 (DC) \\
\hline 05-94 & SH & One IWA, 2 swipes, $\max =0.048$ (DC) \\
\hline 05-AL6 & FO & One IWA, max $=0.046$ (DC) \\
\hline 05-E25175 & FO & One IWA, max = $0.200(\mathrm{DC})=$ DOE RC on window sill; rest $<0.1$ \\
\hline 05-E26303 & FO & One IWA, max = 0.096 (DC) \\
\hline 05-E92081 & FO & One IWA, 2 swipes, max = 0.074 (DC) \\
\hline 05-E92759 & FO & One IWA, $\max =0.040(\mathrm{DC})$ \\
\hline 05-F800 & FO & One IWA, max = 0.009 (BN_adj) \\
\hline $\begin{array}{l}\text { 05-HAZMAT TA } \\
\text { (machine shop) }\end{array}$ & SH & Area UTL $=0.055$ \\
\hline $\begin{array}{l}\text { 05-HAZMAT TA } \\
\text { (storage shed) }\end{array}$ & ST & One IWA, max = $0.170(D C) ;$ rest $<0.1$ \\
\hline $\begin{array}{l}\text { 05-HAZMAT TA } \\
\text { (tool shed) }\end{array}$ & SH & One IWA, max $=0.079(\mathrm{DC})$ \\
\hline 05-SGS TLR & FO & Two IWAs, both sampled, max $=0.123$ (AT) \\
\hline 05-T00114 & FO & One IWA, max = $0.088(\mathrm{DC})$ \\
\hline 05-WEF-HSG & FO & One IWA; Later sampling; max = 0.151 (DC); rest < 0.1 \\
\hline 05-WEF-OFF & FO & One IWA, max $<0.005$ (AT) \\
\hline 05-WEF-RTR & LA & Area UTL $=0.052$ \\
\hline 06-159 & SH & UTL_cluster = 0.100; Area UTL $=0.191$ \\
\hline 06-160 & SH & One IWA, max = 0.015 (BN_adj) \\
\hline 06-270 & FO & One IWA, max = 0.185 (MS_arch, on electrical conduit); rest < 0.1 \\
\hline 06-304 DAF & $\mathbf{E X}$ & One IWA, max = 0.012 (BN_adj) \\
\hline 06-305 DAF & $\mathbf{E X}$ & One IWA, max = 0.065 (MS_arch) \\
\hline 06-310 DAF & EX & One IWA, max = 0.003 (BN-adj) \\
\hline 06-343 DAF & $\mathbf{E X}$ & One IWA, max = 0.017 (MS_arch) \\
\hline 06-345 DAF & SH & One IWA, max = 0.011 (MS_arch) \\
\hline 06-353 DAF & EX & One IWA, max = 0.015 (MS_arch) \\
\hline 06-400 DAF & OF & PLall $=0.010$ \\
\hline 06-500 DAF & SH & All IWAs sampled; max = 0.061 (MS_arch) \\
\hline 06-605 EGS DAF & FO & PLall $=0.128$ \\
\hline 06-610 DAF & $\mathbf{O F}$ & One IWA, max = 0.021 (MS_arch) \\
\hline 06-611 DAF & $\mathbf{O F}$ & One IWA, max = -0.002 (BN_adj) \\
\hline $06-618$ & FO & Area UTL $=0.068$ \\
\hline 06-624 & SH & $\begin{array}{l}\text { IWA PLall = 0.046; } 3 \text { Area swipes }>\text { DOE RC, max = } 0.317 \text { (MS_new), bulk Be and } \\
\text { metal ratios OK }\end{array}$ \\
\hline 06-625 & FO & PLall = 0.174 \\
\hline 06-651 DAF & FO & One IWA, max = 0.008 (MS_arch) \\
\hline 06-652 DAF & FO & One IWA, max = 0.019 (MS_arch) \\
\hline 06-800 & SH & $\begin{array}{l}\text { Both IWAs sampled, } \max =0.088(\text { MS_arch); Area UTL }=1.843 \text { with } 15>\text { DOE RC; } \\
\text { bulks OK, but GW ratios not good for Sheldon lathe }\end{array}$ \\
\hline
\end{tabular}




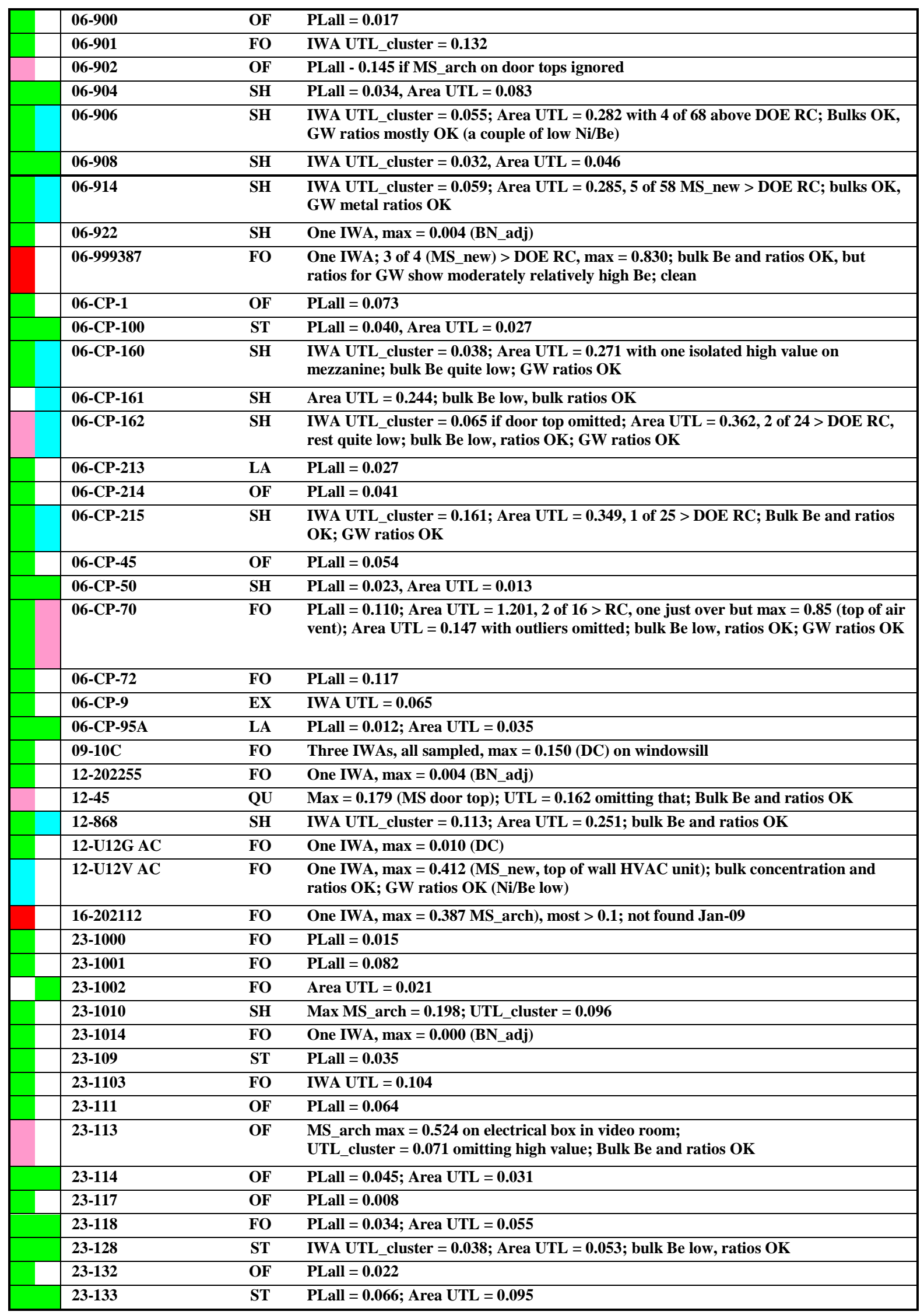




\begin{tabular}{|c|c|c|}
\hline $23-143$ & $\mathbf{O F}$ & PLall = 0.180 \\
\hline $\begin{array}{l}23-151 \& 154 \& \\
156 \& 158\end{array}$ & ST & $\begin{array}{l}\text { IWA UTL_cluster }=0.090,1 \text { of } 42>\text { DOC RC; Area UTL }=0.082,1 \text { of } 62>\text { DOE RC; } \\
\text { bulk Be and ratios OK }\end{array}$ \\
\hline 23-153 & LA & PLall = 0.042; Area UTL = 0.016 \\
\hline 23-159 & OF & All IWAs sampled; $\max =0.001$ (DC) \\
\hline 23-160 & ST & Area UTL $=0.016$ \\
\hline 23-180 & SH & PLall $=0.043$ \\
\hline $23-190$ & FO & $\begin{array}{l}\text { IWA PLall }=0.030 \text {, Area UTL }=0.038 \text { excluding rooms } 19 \text { and } 193 ; 2 \text { of } 12 \text { MS_new in } \\
\text { those rooms }>\text { DOE RC; bulk Be and ratios OK, GW ratios OK }\end{array}$ \\
\hline $23-211$ & FO & PLall = 0.012; Area UTL = 0.012 \\
\hline 23-300 & $\mathbf{Q U}$ & PLall = 0.038, Area UTL = 0.019 \\
\hline 23-302 & ST & One IWA, max = 0.011 (BN_adj) \\
\hline 23-310 & OF & PLall $=0.044$ \\
\hline 23-425 & FO & IWA UTL = 0.104 \\
\hline $\begin{array}{l}23-475 \& 476 \& 478 \\
\& 479 \& 526 \& 527 \\
\& 529\end{array}$ & $\mathbf{Q U}$ & UTL_cluster $=0.026$ \\
\hline $\begin{array}{l}23-480 \& 481 \& 482 \\
\& 483 \& 484\end{array}$ & QU & PLall = 0.075 \\
\hline $23-525$ & OF & PLall = 0.105 \\
\hline $23-528 \& 530$ & $\mathbf{Q U}$ & PLall = 0.171 \\
\hline 23-531 \& $532 \& 535$ & QU & PLall = 0.011 \\
\hline $23-550$ & OF & PLall = 0.028 \\
\hline 23-600 & EX & PLall $=0.085$, Area UTL $=0.002$ \\
\hline 23-610 & FO & $\begin{array}{l}1 \text { of } 21 \text { MS_new > DOE RC (windowsill, 0.314), rest < 0.06; without outlier, Area UTL } \\
\text { = 0.106; bulk Be low, ratios OK, GW ratios OK except Ni relatively low }\end{array}$ \\
\hline 23-614 & OF & PLall = 0.038 \\
\hline 23-620 & OF & PLall $=0.026$ \\
\hline 23-630 & OF & PLall $=0.021$ \\
\hline 23-650 & LA & PLall = 0.010 \\
\hline 23-652 & OF/LA & Office PLall = 0.019; all Lab IWAs sampled; max = 0.044 (DC) \\
\hline $\begin{array}{l}23-675 \& 676 \& 678 \\
\& 679 \& 680 \& 681 \\
\& 683 \& 684\end{array}$ & QU & IWA UTL $=0.124$ \\
\hline 23-700 & SH & PLall = 0.064; Area UTL = 0.011 \\
\hline 23-701 & FO & IWA UTL $=0.118$ \\
\hline 23-703 & ST & $\begin{array}{l}1 \text { of } 59(\mathrm{MS} \text {-new }=0.411)>\text { DOE } \mathrm{RC} \text {; Area } \mathrm{UTL}=0.088 \text {; bulk Be low, ratios OK; GW } \\
\text { ratio has Be high compared with Y }\end{array}$ \\
\hline 23-710 & SH & IWA UTL = 0.186; Area UTL = 0.024 \\
\hline $23-722003$ & ST & One IWA, max = 0.003 (BN_adj) \\
\hline 23-725 & CO & PLall = 0.017 \\
\hline 23-726 & CO & $\begin{array}{l}\text { Top of } \mathrm{RF} \text { room door }>\text { DOE RC; post; IWA UTL }=0.138 \text { even with this value; } \text { PLall } \\
=0.063 \text { without outlier }\end{array}$ \\
\hline 23-750 & SH & PLall $=0.028$, Area UTL $=0.015$ \\
\hline 23-775 \& 776 \& 777 & ST & PLall = 0.073; Area UTL $=0.028$ \\
\hline 23-B & $\mathbf{O F}$ & PLall = 0.011 \\
\hline 23-C & OF & PLall $=0.133$ \\
\hline 23-D & OF & PLall = 0.007 \\
\hline 23-E106727 & FO & $\begin{array}{l}\text { One IWA, max = } 0.698 \text { (MS_arch) top of electrical panel, rest }<0.01 \text {; not sampled Jan- } \\
09\end{array}$ \\
\hline 23-GS100 & FO & One IWA (2 swipes), max = 0.004 (BN_adj) \\
\hline 23-W1 & SH & PLall $=0.094$, Area UTL $=0.053$ \\
\hline 23-W2 & SH & PLall = 0.066, Area UTL = 0.037 \\
\hline 23-W3 & ST & 9 of 18 (MS_arch) > DOE RC; was not resampled Jan 2009 \\
\hline
\end{tabular}




\begin{tabular}{|c|c|c|}
\hline 23-W5 & ST & One IWA, $\max =0.030($ MS-arch); Area UTL $=0.033$ \\
\hline 23-W6 & SH & PLall $=0.170$ \\
\hline 24-A01 & OF & PLall $=0.107$ \\
\hline 24-A02 & OF/ST & PLall = 0.010; Area UTL $=0.005$ \\
\hline $\begin{array}{l}\text { 24-A02 readiness } \\
\text { warehouse }\end{array}$ & ST & NPUTL $=0.200$ \\
\hline 24-A03 & FO & One IWA, $\max =0.003(\mathrm{DC})$ \\
\hline 24-A04 & SH & IWA UTL_cluster = 0.046; Area UTL $=0.010$ \\
\hline 24-A05 & ST & $\begin{array}{l}\text { Max }=0.567>\text { DOE RC }(\text { MS_arch on electrical box }) ; \text { rest }<0.1 ; \\
\text { PLall }=0.095 \text { omitting high value }\end{array}$ \\
\hline 24-A07 & FO & Sampled 2 of 3 IWAs; $\max =0.009$ (DC); UTL_cluster = 0.018 \\
\hline 24-A10 & FO & One IWA, max is $0.005(\mathrm{DC})$ \\
\hline 24-A12 & SH & PLall $=0.007$ \\
\hline 24-A13 & OF & PLall $=0.027$ \\
\hline 24-A15 & SH & Area UTL $=0.026$ \\
\hline 24-B01 & OF & PLall $=0.051$ \\
\hline 24-B02 & OF & PLall $=0.020$ \\
\hline 24-B03 & OF & PLall = 0.046 \\
\hline 24-B04 & FO & Area UTL $=0.113$ \\
\hline 24-B05 & OF & Area UTL $=0.041$ \\
\hline 24-B07 & SH & PLall $=0.022$, Area UTL = 0.001 \\
\hline 24-B09 & ST & One IWA, max = 0.086 (MS_arch) \\
\hline 24-B10 & FO & One IWA, max = $0.006(\mathrm{DC})$ \\
\hline 24-C01 & OF & PLall $=0.021$ \\
\hline 24-C02 & OF/ST & PLall = 0.016, Area UTL = 0.004 \\
\hline 24-C03 & OF & PLall = 0.031 \\
\hline 24-C04 & FO & One IWA, max = 0.003 (DC) \\
\hline 24-NSF & OF & PLall $=0.032$ \\
\hline 25-026107 & FO & One IWA, max = 0.032 (BN_adj) \\
\hline 25-092728 & FO & One IWA, max = 0.082 (MS_arch) \\
\hline $25-096380$ & FO & One IWA, max = 0.034 (BN_adj) \\
\hline $25-096593$ & FO & One IWA, max = 0.030 (BN_adj) \\
\hline 25-097499 & FO & One IWA, max = 0.012 (BN_adj) \\
\hline $25-202616$ & FO & 1 of 18 (MS_new) > DOE RC, max = 0.274; Area UTL = 0.440; GW ratios OK \\
\hline 25-202674 & FO & One IWA, max = 0.072 (MS_new) \\
\hline 25-4117 & FO & PLall $=0.046$ \\
\hline $25-4919$ & FO & UTL_cluster $=0.173$ \\
\hline 25-721949 & FO & Three IWAs, all sampled, max = 0.152 (MS_arch, windowsill), rest $<0.1$ \\
\hline 25-E25169 & FO & One IWA, max = 0.359 (MS_arch) > DOE RC (windowsill), rest < 0.01 \\
\hline 25-SHAWTLR & FO & One IWA, max = 0.003 (BN_adj) \\
\hline 26-185129 & FO & One IWA, max = 0.002 (BN_adj) \\
\hline 26-202558 & FO & One IWA, max = 0.031 (MS_arch) \\
\hline 26-2204 & FO & Area UTL = 0.166; bulk Be and ratios OK \\
\hline $27-250$ & FO & One IWA, max = 0.001 (BN_adj) \\
\hline 27-5100 & $\mathbf{E X}$ & PLall $=0.042$, Area UTL $=0.040$ \\
\hline 27-5110 & ST & $\begin{array}{l}\text { One IWA, MS max }=0.312(\text { MS_arch, DC dup }=0.271)>\text { DOE RC, rest }<\text { RC; bulk } \\
\text { concentrations and ratios OK }\end{array}$ \\
\hline 27-5150 & FO & UTL_cluster $=0.050$ \\
\hline $27-5180$ & FO & Area UTL $=0.044$ \\
\hline 27-5191 & SH & Max = 0.367 (MS_arch) on doortop; omitting that, UTL = 0.051 \\
\hline $27-560$ & FO & One IWA, max = 0.005 (BN_adj) \\
\hline 27-A & FO & One IWA, max = 0.005 (BN_adj) \\
\hline 27-B & FO & UTL_cluster $=0.053$ \\
\hline $\begin{array}{l}\text { 27-SHOWER } \\
\text { TRLR }\end{array}$ & FO & One IWA, max = 0.010 (MS_arch) \\
\hline
\end{tabular}




\begin{tabular}{|c|c|c|}
\hline $35-2211$ & OF & PLall $=0.033$, Area UTL $=0.004$ \\
\hline 35-2215 & ST & Two IWAs, both sampled, max = 0.120 (DC); bulk Be and ratios OK \\
\hline $35-2216$ & ST & Area UTL $=0.040$ \\
\hline $35-2221$ & ST & $\begin{array}{l}\text { PLall }=0.089 \text { w/o outlier; IWA UTL_cluster }=0.039 \text { with all data } \\
\text { Bldg UTL = 0.079; Deployment items UTL }=0.030\end{array}$ \\
\hline $35-2222$ & $\mathbf{E X}$ & PLall = 0.159 \\
\hline $35-2229$ & SH & PLall $=0.022$ \\
\hline 37-FG3\&4 & $\mathbf{O F}$ & PLall = 0.029 \\
\hline 52-0378 \& 0379 & FO & All IWAs sampled; max = 0.062 (DC, windowsill) \\
\hline BN-LAO & LA & PLall = 0.032 \\
\hline BN-LLO & EX & IWA UTL = 0.080 \\
\hline BN-RSLA & FO & Area UTL $=0.040$ \\
\hline
\end{tabular}

\section{Detailed Results by Facility}

The smallest facilities consisting of one or a few IWAs (the fourth case above) are omitted from the following list. Otherwise, for each facility a table of summary statistics and results is presented, along with a plot of the data obtained from that facility. A detailed description is given for each novel element when it first appears.

\section{1-181769}

\begin{tabular}{|c|c|}
\hline \multirow{2}{*}{\multicolumn{2}{|c|}{ 01-181769 }} \\
\hline & \\
\hline Type & OF \\
\hline $\mathrm{N}=$ & 10 \\
\hline $\mathrm{n}=$ & 5 \\
\hline $\mathrm{BC}(\mathrm{BNadj})=$ & -0.0062 \\
\hline delta $=$ & 0.005 \\
\hline mean of $\ln (x+$ delta $)=$ & -5.411 \\
\hline $\ln \mathrm{sd}=$ & 0.445 \\
\hline PL K = & 3.788 \\
\hline PLall = & 0.019 \\
\hline UTL K = & 4.203 \\
\hline UTL = & 0.024 \\
\hline Alpha $=$ & 0.05 \\
\hline ProbPlot $\mathrm{R}=$ & 0.923 \\
\hline
\end{tabular}

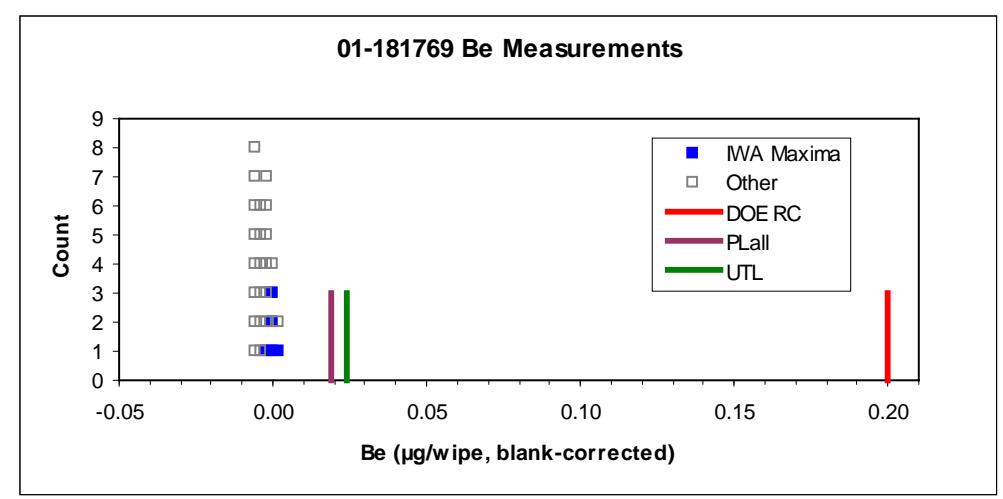

In this table $\mathrm{N}$ is the total number of IWAs (see Appendix 1) and $\mathrm{n}$ is the number of IWAs sampled. BC is the blank correction used to make the low-end distributions of data from different labs, etc., more nearly similar, and delta is the shift added to accommodate the negative values found in the data; see Appendix 4 for discussions of these elements. PLall is a prediction limit for the largest value to be found in all unsampled IWAs. Comparing PLall with the DOE RC is a more stringent test than using the UTL; the latter is recommended in the current draft DOE Technical Standard. Alpha is the significance level of the test implicitly used when comparing PLall or UTL with the DOE RC, and ProbPlot R is the normal probability plot correlation on the transformed scale.

Only the IWA maximum values are used in the computations. The plot shows these as solid squares and the other data has outline squares. Also shown are the RC, PLall, and UTL. For this facility, all data as well as PLall and UTL are far below RC; this facility is clearly "clean." 


\section{1-201959}

\begin{tabular}{|c|c|}
\hline \multicolumn{2}{|l|}{ 01-201959 } \\
\hline Area & \\
\hline Type & $\mathrm{SH}$ \\
\hline $\mathrm{n}=$ & 10 \\
\hline BC(MS_new) $=$ & 0.0001 \\
\hline delta $=$ & 0.0085 \\
\hline mean of $\ln (x+$ delta $)=$ & -2.926 \\
\hline $\ln \mathrm{sd}=$ & 0.921 \\
\hline UTL K = & 2.911 \\
\hline UTL = & 0.774 \\
\hline Alpha $=$ & 0.05 \\
\hline ProbPlot $\mathrm{R}=$ & 0.988 \\
\hline
\end{tabular}

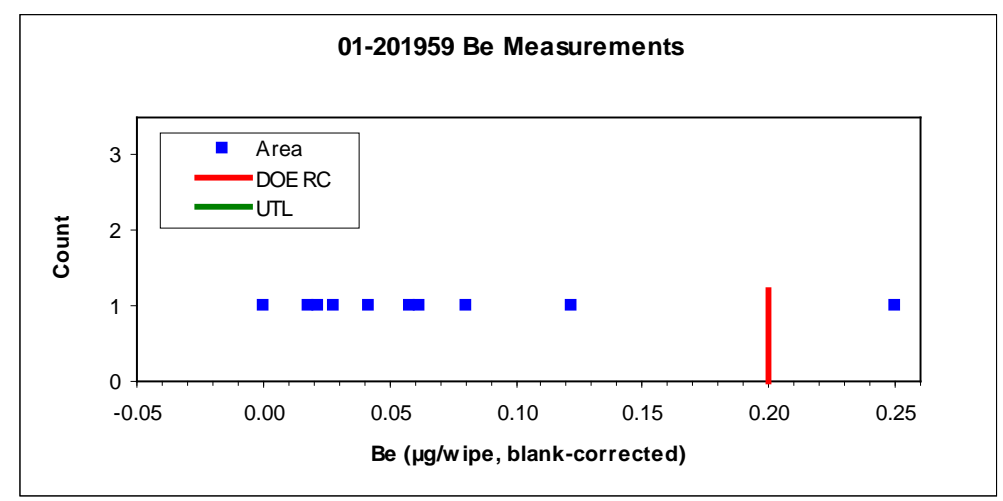

This is a small Area facility, using the UTL approach initially. In this case the largest data value itself ( 0.2495 blank-corrected) exceeds the RC and UTL $=0.774$ is well above it. This facility does not "pass" the UTL test. One bulk sample was obtained; its Be concentration is 1.007, about in the middle of the distribution of soil measurements (actually toward the lower end for samples in the north). The metal ratios in the bulk sample are not remarkable; $\mathrm{Nb} / \mathrm{Be}$ is a bit on the high side, indicating relatively low Be. In the high wipe itself, four of six metal ratios are somewhat high. This facility is given the "blue" pass, meaning that it fails the statistical test and has a sample exceeding the DOE RC, but that there is no evidence to indicate that the high value(s) are not from naturally occurring soils.

\section{1-202655}

\begin{tabular}{|c|c|}
\hline \multirow{2}{*}{\multicolumn{2}{|c|}{ 01-202655 }} \\
\hline & \\
\hline Type & FO \\
\hline $\mathrm{n}=$ & 12 \\
\hline $\mathrm{BC}(\mathrm{BNadj})=$ & -0.0062 \\
\hline $\mathrm{BC}(\mathrm{DC})=$ & 0.0013 \\
\hline delta $=$ & 0.0080 \\
\hline mean of $\ln (x+$ delta $)=$ & -5.129 \\
\hline $\ln \mathrm{sd}=$ & 0.774 \\
\hline UTL K = & 2.736 \\
\hline UTL $=$ & 0.041 \\
\hline Alpha $=$ & 0.05 \\
\hline ProbPlot $\mathrm{R}=$ & 0.954 \\
\hline
\end{tabular}

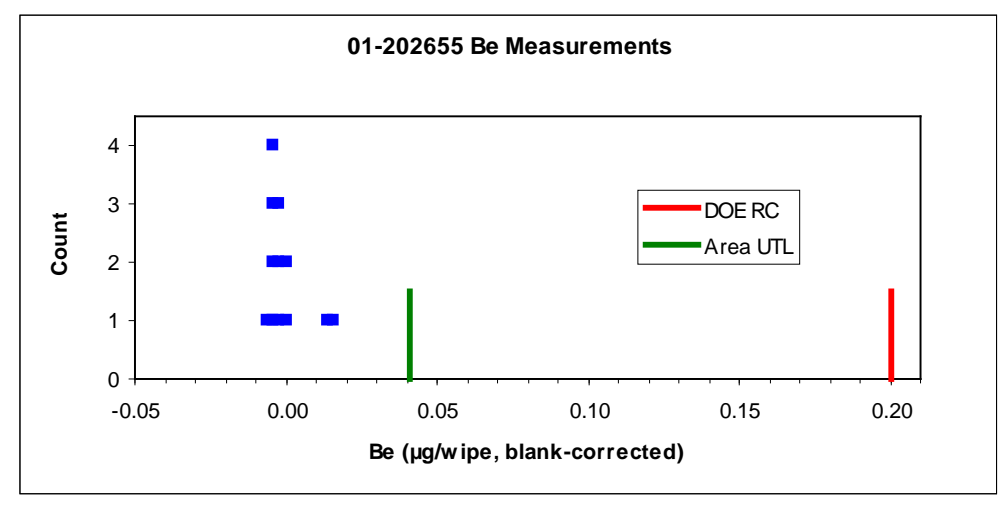

For this facility the Area UTL is not greater than the DOE RC; this facility "passes." 


\section{1-202772}

\begin{tabular}{|c|c|}
\hline \multirow{2}{*}{\multicolumn{2}{|c|}{ 01-202772 }} \\
\hline & \\
\hline Type & $\mathrm{OF}$ \\
\hline $\mathrm{N}=$ & 9 \\
\hline $\mathrm{n}=$ & 5 \\
\hline $\mathrm{BC}(\mathrm{BNadj})=$ & -0.0062 \\
\hline BC(MS_arch) $=$ & 0.0004 \\
\hline delta $=$ & 0.0040 \\
\hline mean of $\ln (x+$ delta $)=$ & -4.743 \\
\hline $\ln \mathrm{sd}=$ & 1.194 \\
\hline $\mathrm{PL} \mathrm{K}=$ & 3.583 \\
\hline PLall $=$ & 0.625 \\
\hline UTL K = & 4.203 \\
\hline UTL = & 1.313 \\
\hline Alpha $=$ & 0.05 \\
\hline ProbPlot $\mathrm{R}=$ & 0.945 \\
\hline \multicolumn{2}{|c|}{ UTL cluster sampling analysis } \\
\hline $\mathrm{F}=$ & 0.37 \\
\hline delta $=$ & 0.0085 \\
\hline mean of $\ln (x+$ delta $)=$ & -5.094 \\
\hline $\ln \mathrm{sd}=$ & 0.681 \\
\hline UTL K = & 2.232 \\
\hline UTL $=$ & 0.020 \\
\hline ProbPlot $\mathrm{R}=$ & 0.974 \\
\hline
\end{tabular}

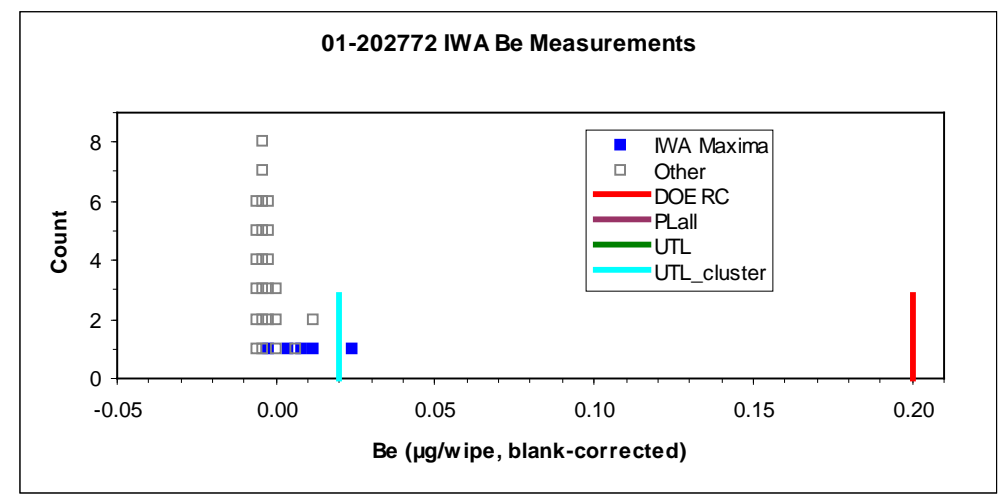

This facility contains only 9 IWAs, of which 5 were sampled. With so few IWAs sampled both PLall and UTL exceed the $\mathrm{RC}$, even though all the data values are far below. The UTL cluster analysis uses all the observations, not only the IWA maxima; UTL_cluster $=0.020$, far less than the RC. The F is from the analysis of variance; with $\mathrm{F}$ this low there is no evidence for positive correlation among measurements obtained in the same IWAs.

\section{1-U1H LR}

\begin{tabular}{|c|c|}
\hline \multicolumn{2}{|l|}{ 01-U1H LR } \\
\hline Area & \\
\hline Type & FO \\
\hline $\mathrm{n}=$ & 10 \\
\hline BCt $($ BNadj $)=$ & -0.0062 \\
\hline $\mathrm{BC}(\mathrm{DC})=$ & 0.0013 \\
\hline delta $=$ & 0.008 \\
\hline mean of $\ln (x+$ delta $)=$ & -5.092 \\
\hline $\ln \mathrm{sd}=$ & 0.439 \\
\hline UTL K = & 2.911 \\
\hline UTL $=$ & 0.014 \\
\hline Alpha $=$ & 0.05 \\
\hline ProbPlot $\mathrm{R}=$ & 0.964 \\
\hline
\end{tabular}

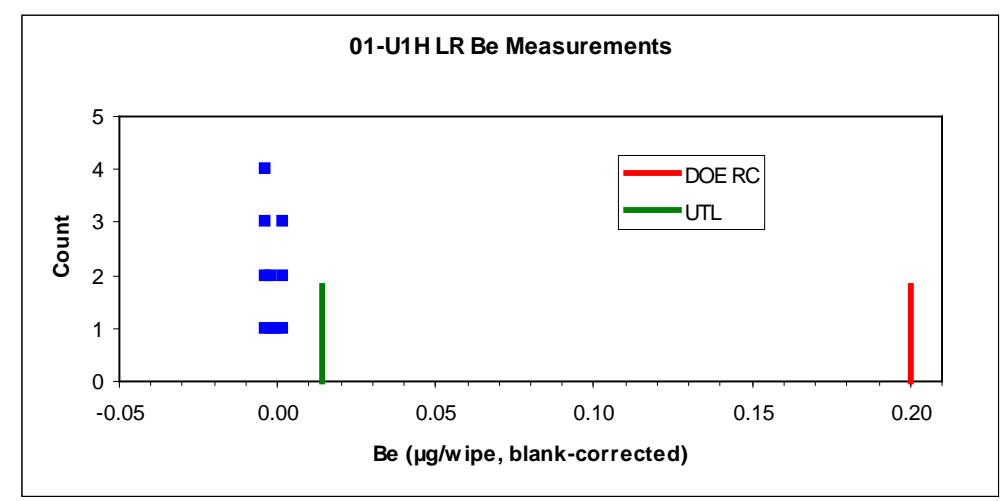




\section{3-3C36}

\begin{tabular}{|c|c|}
\hline \multicolumn{2}{|l|}{ 03-3С 36} \\
\hline \multicolumn{2}{|l|}{ IWAs } \\
\hline Type & OF \\
\hline $\mathrm{N}=$ & 10 \\
\hline $\mathrm{n}=$ & 6 \\
\hline $\mathrm{BC}(\mathrm{BNadj})=$ & -0.0062 \\
\hline BC(MS_new) $=$ & 0.0001 \\
\hline $\mathrm{BC}(\mathrm{DC})=$ & 0.0013 \\
\hline delta $=$ & 0.0040 \\
\hline mean of $\ln (x+$ delta $)=$ & -3.492 \\
\hline $\ln \mathrm{sd}=$ & 2.460 \\
\hline PL K = & 3.259 \\
\hline PLall = & 92.137 \\
\hline UTL K = & 3.708 \\
\hline UTL = & 278.039 \\
\hline Alpha $=$ & 0.05 \\
\hline ProbPlot $\mathrm{R}=$ & 0.950 \\
\hline \multicolumn{2}{|c|}{ omitting high values } \\
\hline mean of $\ln (x+$ delta $)=$ & -4.616 \\
\hline $\ln \mathrm{sd}=$ & 1.498 \\
\hline PLall $=$ & 1.298 \\
\hline UTL $=$ & 2.547 \\
\hline ProbPlot $\mathrm{R}=$ & 0.970 \\
\hline \multicolumn{2}{|c|}{$\begin{array}{l}\text { UTL cluster sampling analysis } \\
\text { omitting high values }\end{array}$} \\
\hline $\mathrm{F}=$ & 0.92 \\
\hline delta $=$ & 0.0085 \\
\hline mean of $\ln (\mathrm{x}+$ delta $)=$ & -4.759 \\
\hline $\ln \mathrm{sd}=$ & 1.011 \\
\hline UTL K = & 2.246 \\
\hline UTL $=$ & 0.075 \\
\hline ProbPlot $\mathrm{R}=$ & 0.851 \\
\hline
\end{tabular}

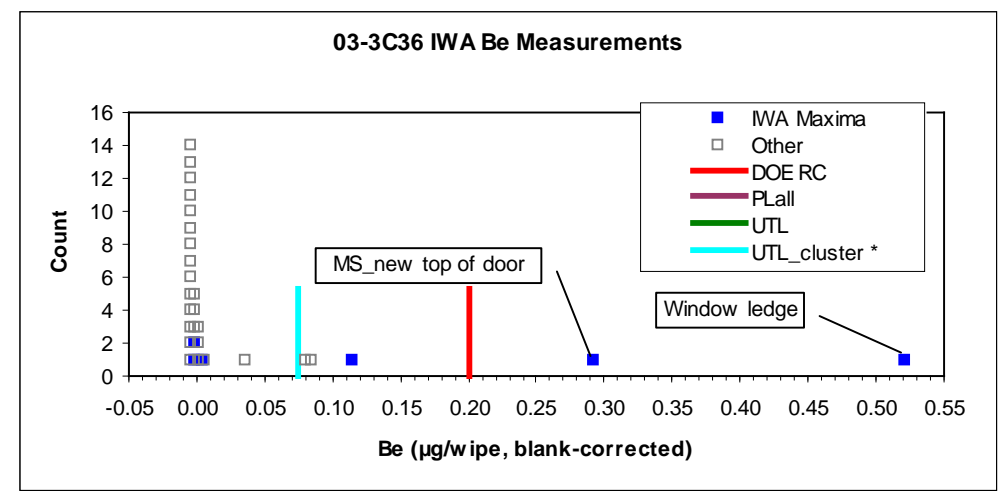

In this facility there are two isolated high values, both MS_new, that drive PLall and UTL up. Even with those omitted values there are three right-tail outliers. This is a facility in a forward area which is likely to be dusty.

If one omits the two highest values and performs the UTL cluster sampling analysis, the result is a pass.

The Be concentration in the bulk sample obtained in this facility is consistent with background soils in the north of the NTS, as are the metal ratios in the bulk sample. The metal ratios in the highest two wipes are OK except for very low ratios of Ni/Be [nickel/beryllium].

The ProbPlot $\mathrm{R}$ is low due to the three remaining right-tail outliers. 


\section{5-24}

\begin{tabular}{|c|c|c|}
\hline \multirow{2}{*}{\multicolumn{3}{|c|}{$05-24$}} \\
\hline & & \\
\hline & Type & ST \\
\hline & $\mathrm{n}=$ & 21 \\
\hline & $\mathrm{BC}(\mathrm{AT})=$ & 0 \\
\hline & delta $=$ & 0 \\
\hline & mean of $\ln (x+$ delta $)=$ & -2.926 \\
\hline & $\ln \mathrm{sd}=$ & 0.858 \\
\hline & UTL K = & 2.371 \\
\hline & UTL = & 0.410 \\
\hline & Alpha = & 0.05 \\
\hline & ProbPlot $\mathrm{R}=$ & 0.982 \\
\hline
\end{tabular}

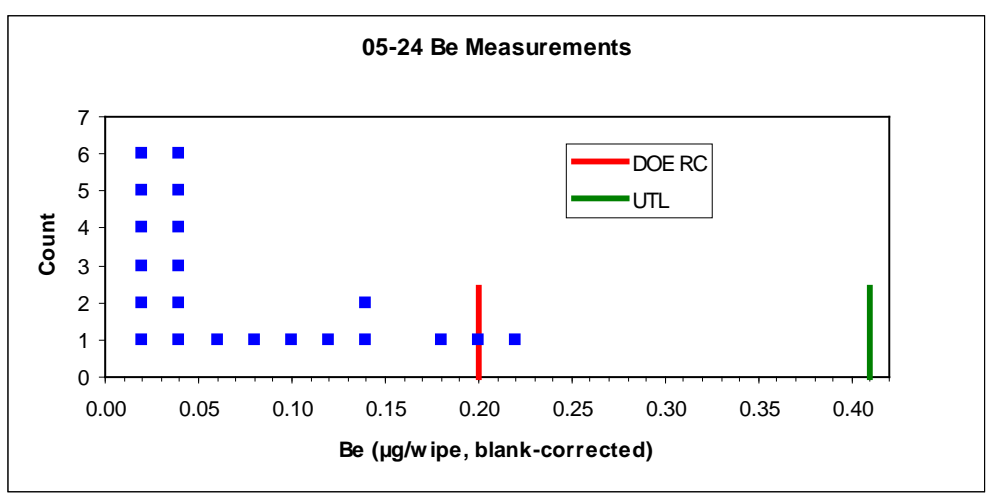

This is a storage facility for transuranic (TRU) waste in Area 5, and is inherently dusty. The high Be values were located around the periphery, as shown in the adjacent plot.

Two bulk samples were obtained inside the facility during the January 2009 sampling. Be concentrations and metal ratios for these are consistent with those of NTS soils.

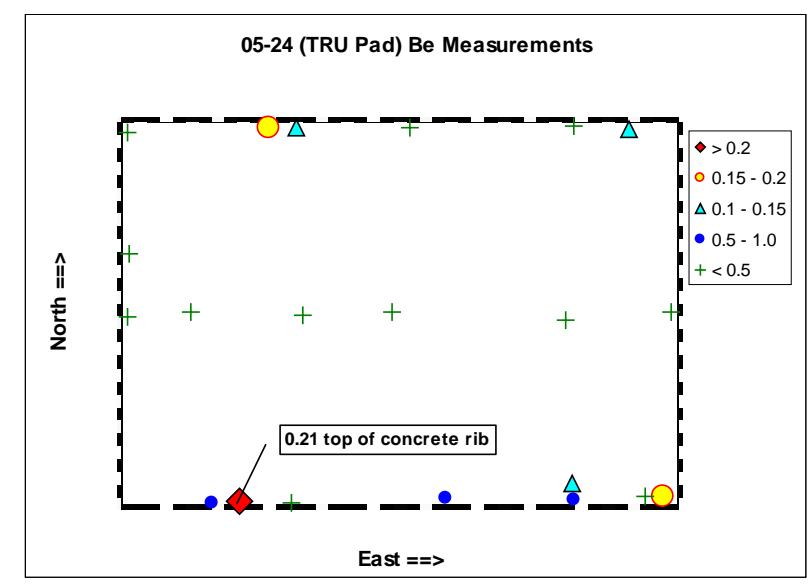

05-31

\begin{tabular}{|c|c|c|}
\hline \multicolumn{3}{|l|}{ 05-31 } \\
\hline & IWAs & \\
\hline & Type & OF \\
\hline & $\mathrm{N}=$ & 15 \\
\hline & $\mathrm{n}=$ & 8 \\
\hline & $\mathrm{BC}(\mathrm{BNadj})=$ & -0.0062 \\
\hline & $\mathrm{BC}(\mathrm{DC})=$ & 0.0013 \\
\hline & delta $=$ & 0.005 \\
\hline & mean of $\ln (\mathrm{x}+$ delta $)=$ & -4.611 \\
\hline & $\ln \mathrm{sd}=$ & 0.704 \\
\hline & PL K = & 3.288 \\
\hline & PLall = & 0.096 \\
\hline & UTL K = & 3.187 \\
\hline & UTL = & 0.089 \\
\hline & Alpha $=$ & 0.05 \\
\hline & ProbPlot R = & 0.978 \\
\hline
\end{tabular}

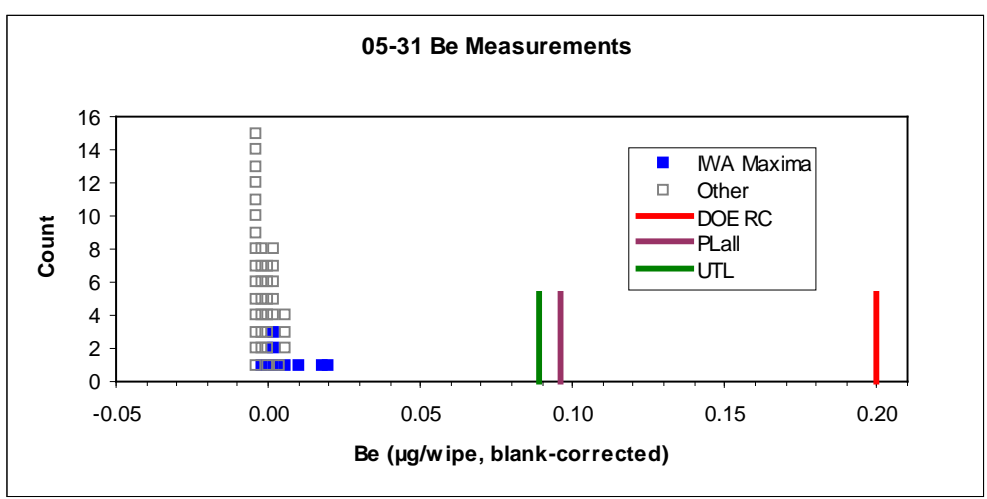


05-32

\begin{tabular}{|c|c|c|}
\hline \multirow{2}{*}{\multicolumn{3}{|c|}{ 05-32 }} \\
\hline & & \\
\hline & Type & EX \\
\hline & $\mathrm{n}=$ & 41 \\
\hline & $R L=$ & 0.005 \\
\hline & mean of $\ln (\mathrm{x})=$ & -5.188 \\
\hline & $\ln \mathrm{sd}=$ & 0.832 \\
\hline & UTL K = & 2.229 \\
\hline & UTL = & 0.036 \\
\hline & Alpha $=$ & 0.05 \\
\hline & ProbPlot $\mathrm{R}=$ & 0.939 \\
\hline
\end{tabular}

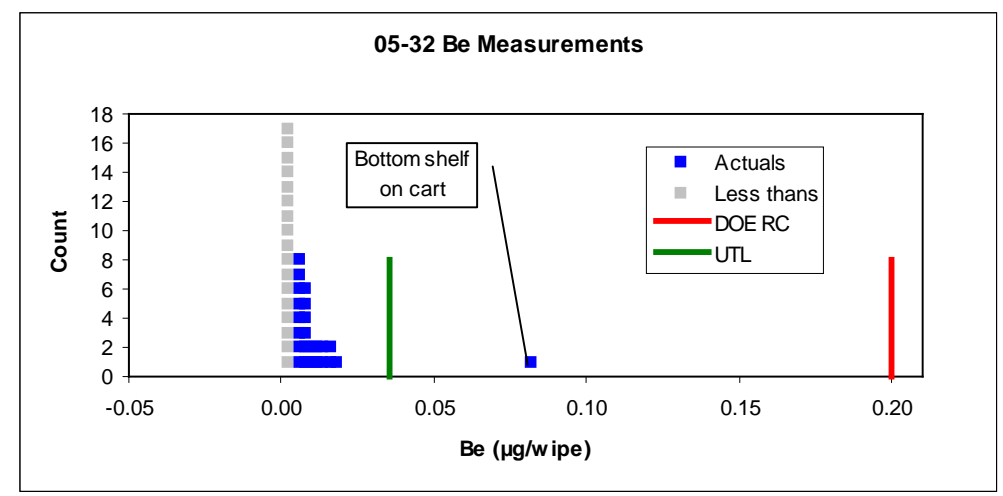

In a few cases with Assay Technology (AT) data or early DataChem data, there are some censored values given as " $<\mathrm{RL}$," where $\mathrm{RL}$ is a reporting limit, or as " $<0$," where the actual negative values were not located. Censored data maximum likelihood estimation is used for these; see "Parametric 95\%-95\% Upper Tolerance Limits for Censored Lognormal Data" (C. B. Davis, presented at the Joint Statistical Meetings, Seattle, 2006). A calibration factor (CF) for the UTL K multipliers was presented in that paper; its function is to accommodate the loss of information resulting from the censoring. The same CF was used for the PLall K multipliers. Censored values are represented in the plot by hollow blue squares at a value half their RL, where RL $>0$, or -0.002 , if “ $<0$.”

\section{5-652B}

\begin{tabular}{|c|c|}
\hline \multicolumn{2}{|l|}{ 05-652B } \\
\hline Area & \\
\hline Type & FO \\
\hline $\mathrm{n}=$ & 14 \\
\hline $\mathrm{BC}(\mathrm{DC})=$ & 0.0003 \\
\hline delta $=$ & 0.0055 \\
\hline mean of $\ln (x+$ delta $)=$ & -4.577 \\
\hline $\ln \mathrm{sd}=$ & 0.486 \\
\hline UTL K = & 2.614 \\
\hline UTL = & 0.031 \\
\hline Alpha $=$ & 0.05 \\
\hline ProbPlot $\mathrm{R}=$ & 0.985 \\
\hline
\end{tabular}

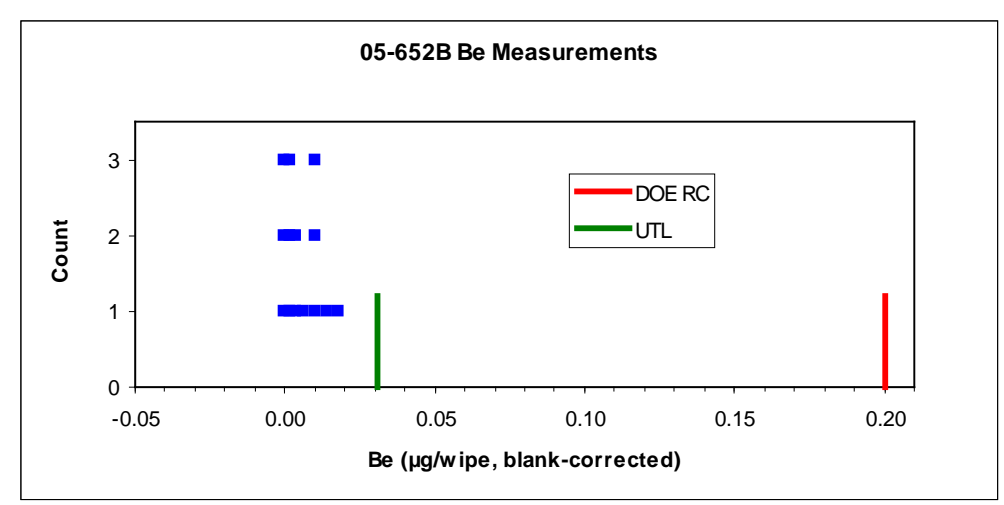


05-7

0

\begin{tabular}{rc|} 
IWAs & \\
Type & OF \\
$\mathrm{N}=$ & 25 \\
$\mathrm{n}=$ & 13 \\
BC(BNadj) $=$ & -0.0062 \\
BC $($ DC $)=$ & 0.0013 \\
delta $=$ & 0.0050 \\
mean of $\ln (\mathrm{x}+$ delta $)=$ & -4.710 \\
$\ln \mathrm{sd}=$ & 0.306 \\
$\mathrm{PL} \mathrm{K}=$ & 3.141 \\
PLall $=$ & $\mathbf{0 . 0 1 9}$ \\
UTL K $=$ & 2.671 \\
UTL $=$ & $\mathbf{0 . 0 1 5}$ \\
Alpha $=$ & 0.05 \\
ProbPlot $\mathrm{R}=$ & 0.993
\end{tabular}

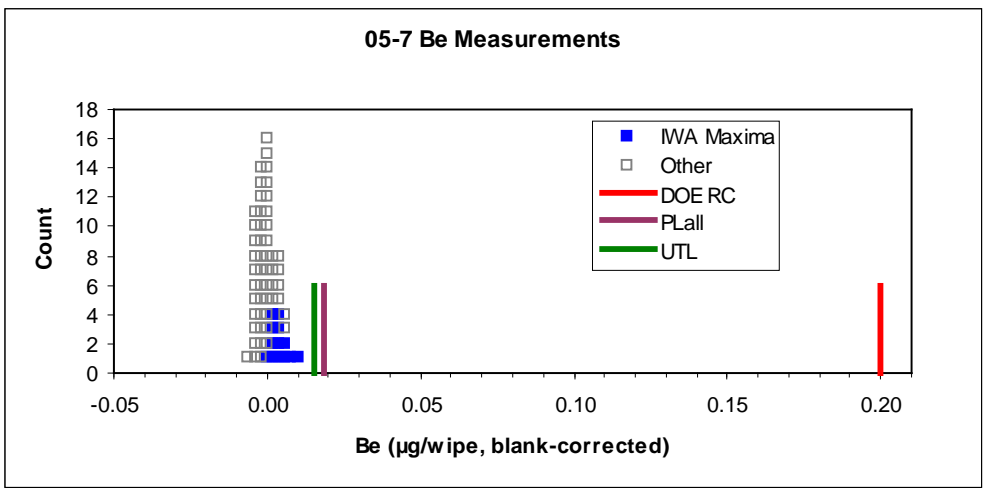

05-8

\begin{tabular}{|c|c|c|}
\hline \multirow[t]{15}{*}{ 05-8 } & & \\
\hline & IWAs & \\
\hline & Type & EX \\
\hline & $\mathrm{N}=$ & 19 \\
\hline & $\mathrm{n}=$ & 6 \\
\hline & $\mathrm{BC}(\mathrm{DC})=$ & 0.0003 \\
\hline & delta $=$ & 0.0020 \\
\hline & mean of $\ln (x+$ delta $)=$ & -4.881 \\
\hline & $\ln \mathrm{sd}=$ & 0.762 \\
\hline & PL K = & 4.172 \\
\hline & PLall = & 0.181 \\
\hline & UTL K = & 3.708 \\
\hline & UTL $=$ & 0.126 \\
\hline & Alpha = & 0.05 \\
\hline & ProbPlot $\mathrm{R}=$ & 0.944 \\
\hline
\end{tabular}

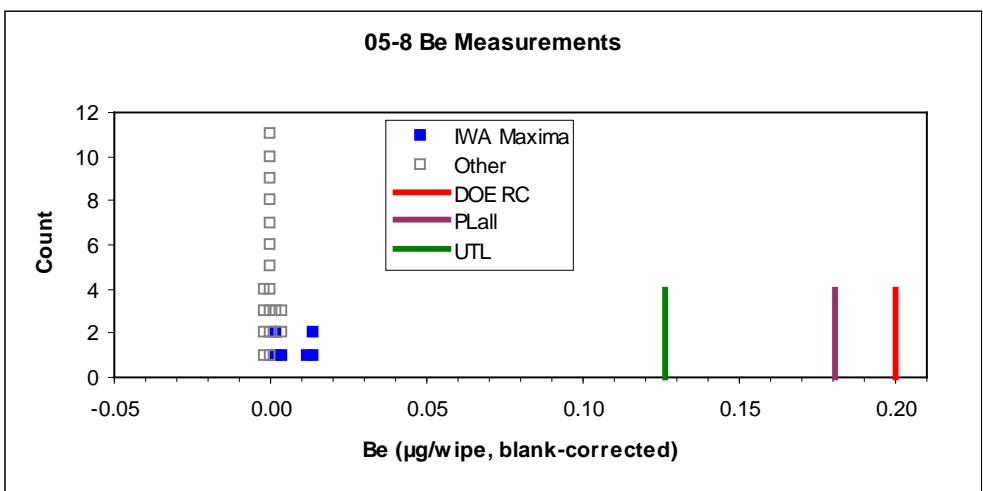

PLall and UTL are rather far above the data values due to the fairly small number of IWAs sampled. Both are less than the DOE RC nonetheless. 


\section{5-HAZMAT Machine Shop}

\begin{tabular}{|c|c|}
\hline \multicolumn{2}{|l|}{ 05-HZMT-Machine Shop } \\
\hline Area & \\
\hline Type & SH \\
\hline $\mathrm{n}=$ & 11 \\
\hline $\mathrm{BC}(\mathrm{DC})=$ & 0.0003 \\
\hline delta $=$ & 0.0055 \\
\hline mean of $\ln (\mathrm{x}+$ delta $)=$ & -4.271 \\
\hline $\ln \mathrm{sd}=$ & 0.521 \\
\hline UTL K = & 2.815 \\
\hline UTL = & 0.055 \\
\hline Alpha $=$ & 0.05 \\
\hline ProbPlot $\mathrm{R}=$ & 0.888 \\
\hline
\end{tabular}

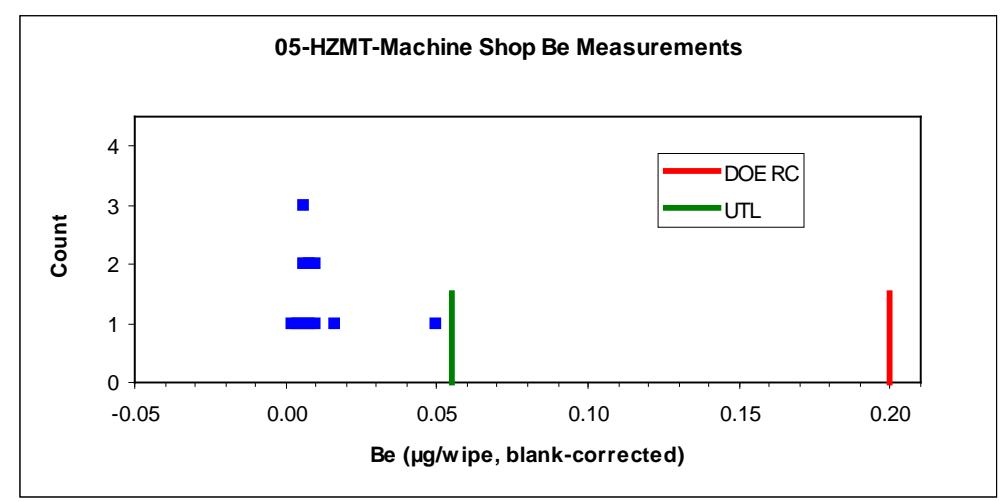

The somewhat low ProbPlot $\mathrm{R}$ is due to the one relatively high value in an otherwise rather clean facility.

\section{5-WEF RTR}

\begin{tabular}{|c|c|}
\hline \multirow{2}{*}{\multicolumn{2}{|c|}{ 05-WEF-RTR }} \\
\hline Area & \\
\hline Type & LA \\
\hline $\mathrm{n}=$ & 11 \\
\hline $\mathrm{BC}(\mathrm{AT})=$ & 0 \\
\hline delta $=$ & 0 \\
\hline mean of $\ln (x+$ delta $)=$ & -4.427 \\
\hline $\ln \mathrm{sd}=$ & 0.521 \\
\hline UTL K = & 2.815 \\
\hline UTL = & 0.052 \\
\hline Alpha $=$ & 0.05 \\
\hline ProbPlot $\mathrm{R}=$ & 0.943 \\
\hline
\end{tabular}

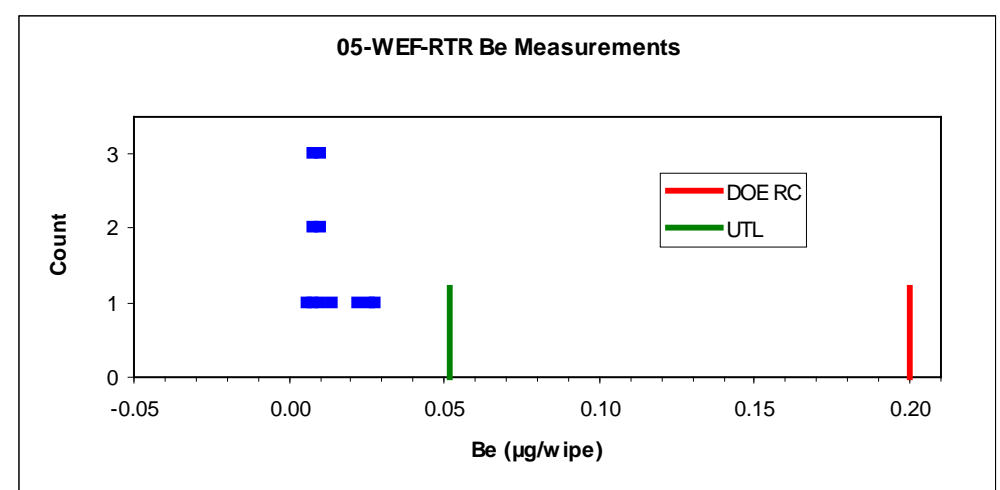




\section{6-159}

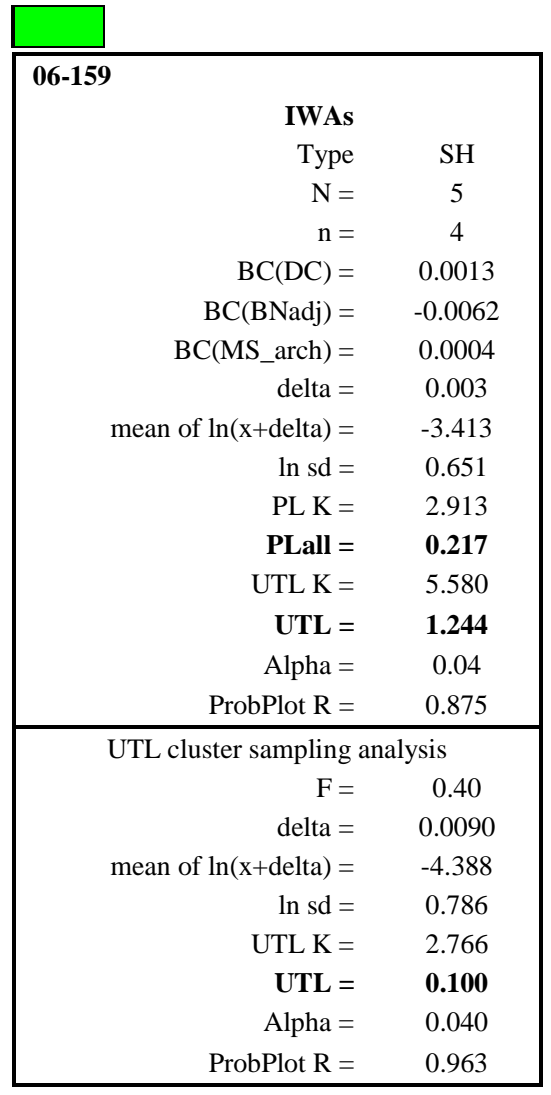

\begin{tabular}{|c|c|}
\hline \multicolumn{2}{|l|}{ 06-159 } \\
\hline Area & \\
\hline Type & SH \\
\hline $\mathrm{n}=$ & 44 \\
\hline $\mathrm{BC}(\mathrm{DC})=$ & 0.0013 \\
\hline $\mathrm{BC}(\mathrm{BNadj})=$ & -0.0062 \\
\hline BC(MS_arch) = & 0.0004 \\
\hline BC(MS_new) = & 0.0001 \\
\hline delta $=$ & 0.0085 \\
\hline mean of $\ln (x+$ delta $)=$ & -3.587 \\
\hline $\ln \mathrm{sd}=$ & 0.926 \\
\hline UTL K = & 2.321 \\
\hline UTL $=$ & 0.191 \\
\hline Alpha $=$ & 0.01 \\
\hline ProbPlot $\mathrm{R}=$ & 0.991 \\
\hline
\end{tabular}

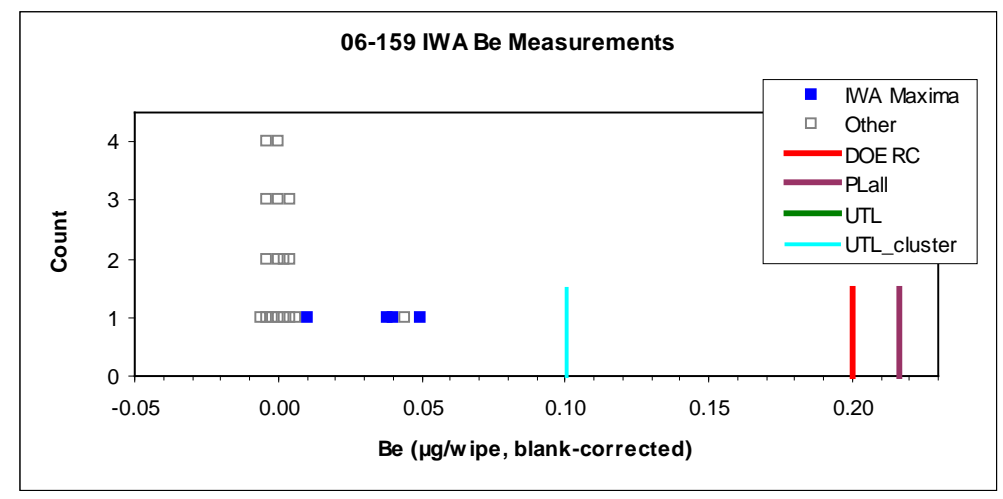

This facility consists of both IWA and Area portions. These are handled separately. The significance levels for the two statistical tests (alpha values) add up to 0.05.

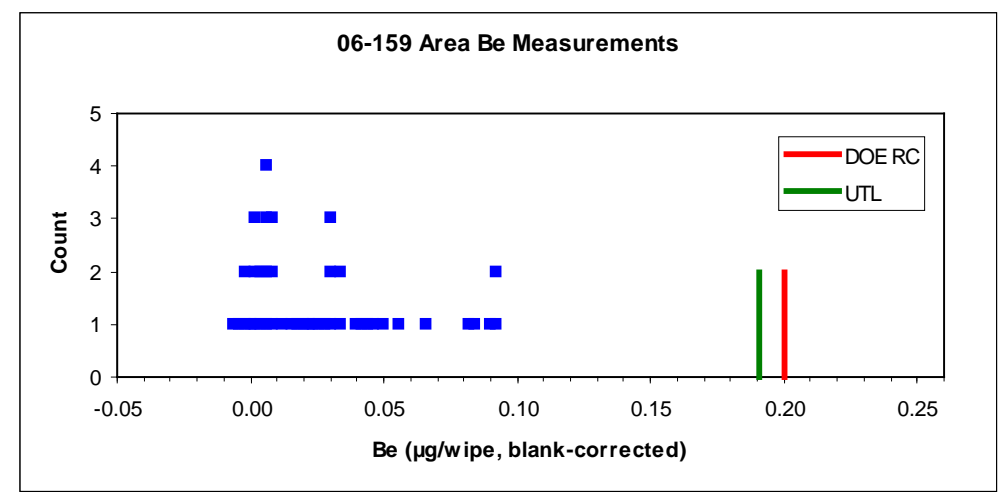




\section{6-400 DAF}

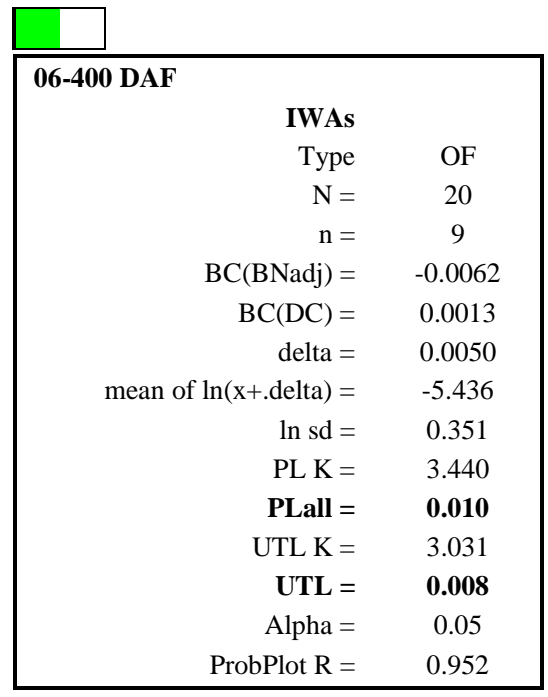

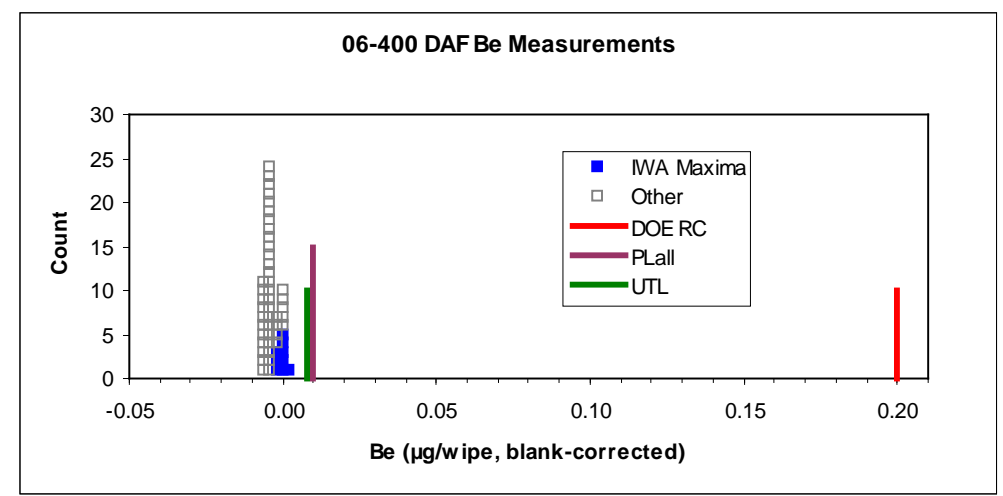

\section{6-605 EGS DAF}

\begin{tabular}{|c|c|}
\hline \multicolumn{2}{|l|}{ 06-605 EGS DAF } \\
\hline IWAs & \\
\hline Tyре & FO \\
\hline $\mathrm{N}=$ & 5 \\
\hline $\mathrm{n}=$ & 4 \\
\hline BC(BNadj $)=$ & -0.0062 \\
\hline BC(MS_arch $)=$ & 0.0004 \\
\hline delta $=$ & 0.0030 \\
\hline mean of $\ln (\mathrm{x}+$ delta $)=$ & -4.211 \\
\hline $\ln \mathrm{sd}=$ & 0.827 \\
\hline PL K = & 2.631 \\
\hline PLall = & 0.128 \\
\hline UTL K = & 5.144 \\
\hline UTL = & 1.041 \\
\hline Alpha $=$ & 0.05 \\
\hline ProbPlot $\mathrm{R}=$ & 0.811 \\
\hline
\end{tabular}

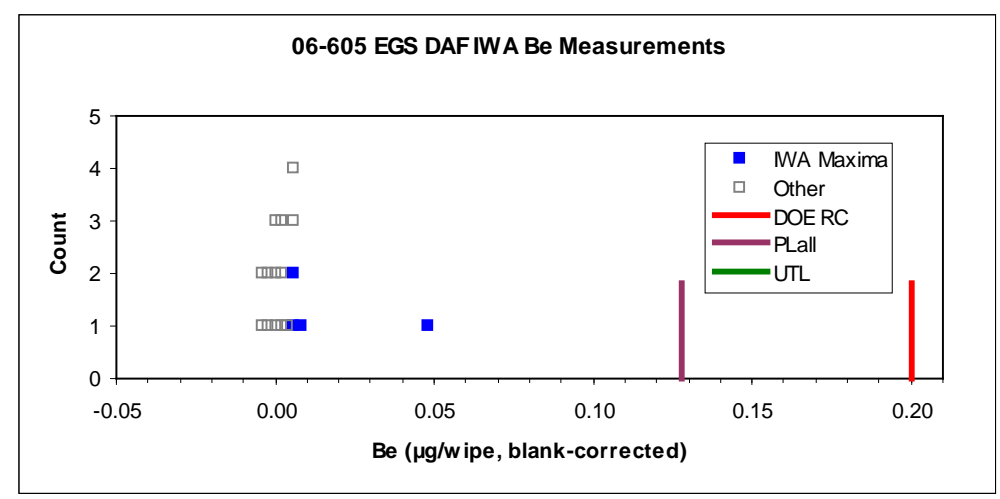

The low ProbPlot $\mathrm{R}$ is due to one of four IWA maxima being higher than the rest, even though all are far below the DOE RC. 
06-618

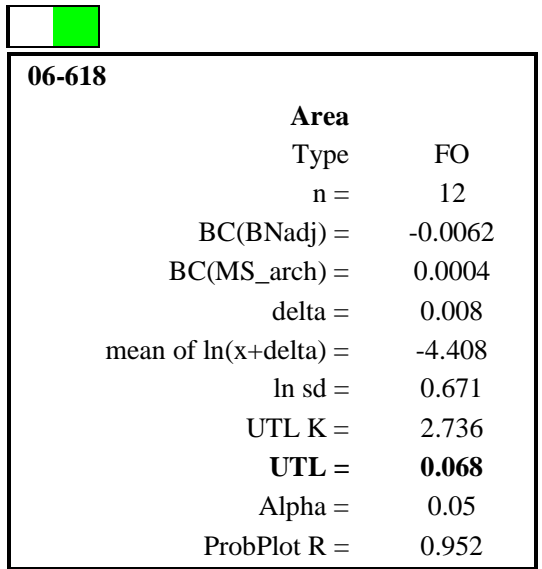

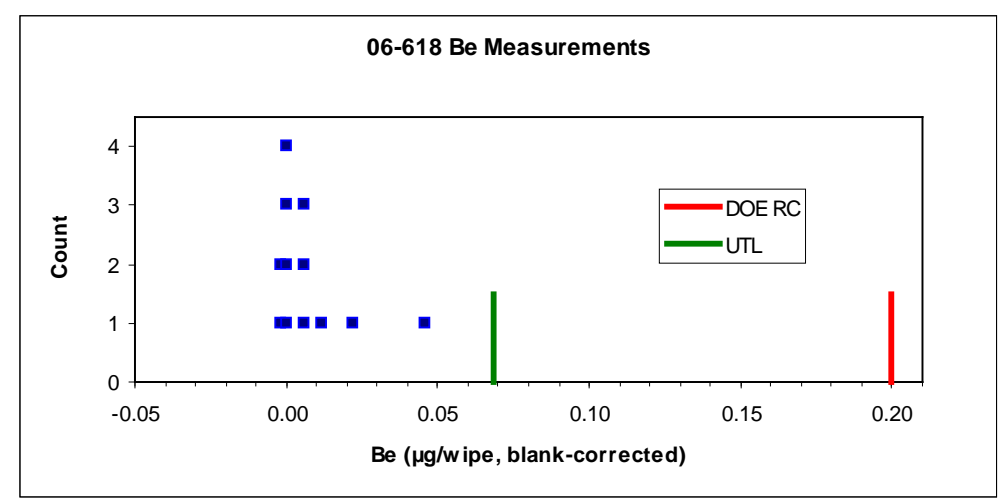

\section{6-624}

\section{$\square$}

\begin{tabular}{|c|c|}
\hline \multicolumn{2}{|l|}{$06-624$} \\
\hline \multicolumn{2}{|l|}{ IWAs } \\
\hline Type & SH \\
\hline $\mathrm{N}=$ & 7 \\
\hline $\mathrm{n}=$ & 4 \\
\hline $\mathrm{BC}(\mathrm{BNadj})=$ & -0.0062 \\
\hline $\mathrm{BC}(\mathrm{DC})=$ & 0.0013 \\
\hline delta $=$ & 0.003 \\
\hline mean of $\ln (x+$ delta $)=$ & -4.963 \\
\hline $\ln \mathrm{sd}=$ & 0.386 \\
\hline $\mathrm{PL} \mathrm{K}=$ & 5.068 \\
\hline PLall $=$ & 0.046 \\
\hline UTL K = & 6.602 \\
\hline UTL = & 0.086 \\
\hline Alpha $=$ & 0.025 \\
\hline ProbPlot $\mathrm{R}=$ & 0.933 \\
\hline \multicolumn{2}{|l|}{ 06-624 } \\
\hline Area & \\
\hline Type & $\mathrm{SH}$ \\
\hline $\mathrm{n}=$ & 46 \\
\hline $\mathrm{BC}(\mathrm{BNadj})=$ & -0.0062 \\
\hline $\mathrm{BC}(\mathrm{DC})=$ & 0.0013 \\
\hline BC(MS_new $)=$ & 0.0001 \\
\hline delta $=$ & 0.0085 \\
\hline mean of $\ln (x+$ delta $)=$ & -3.281 \\
\hline $\ln \mathrm{sd}=$ & 0.963 \\
\hline UTL K = & 2.183 \\
\hline UTL = & 0.305 \\
\hline Alpha $=$ & 0.025 \\
\hline ProbPlot $\mathrm{R}=$ & 0.986 \\
\hline
\end{tabular}
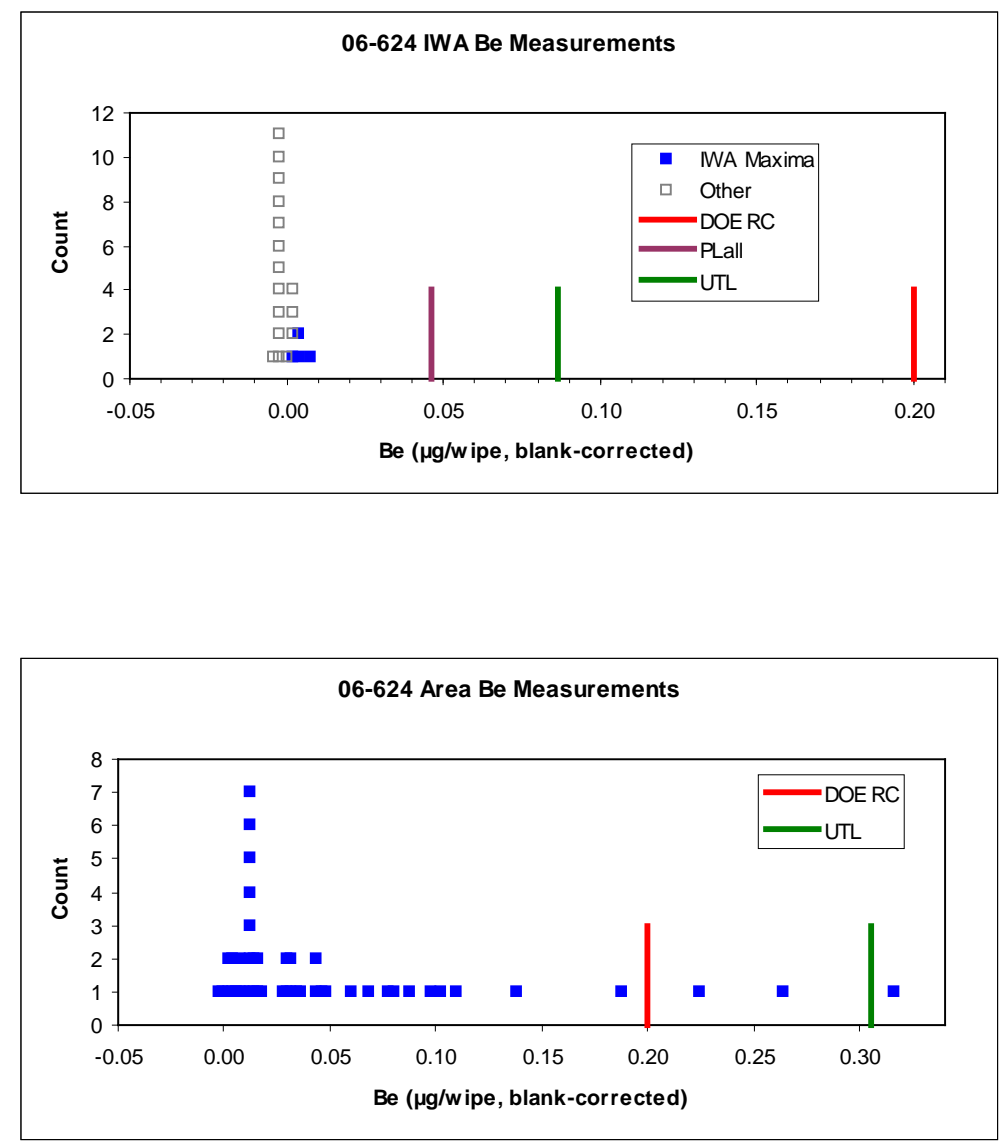


\section{6-625}

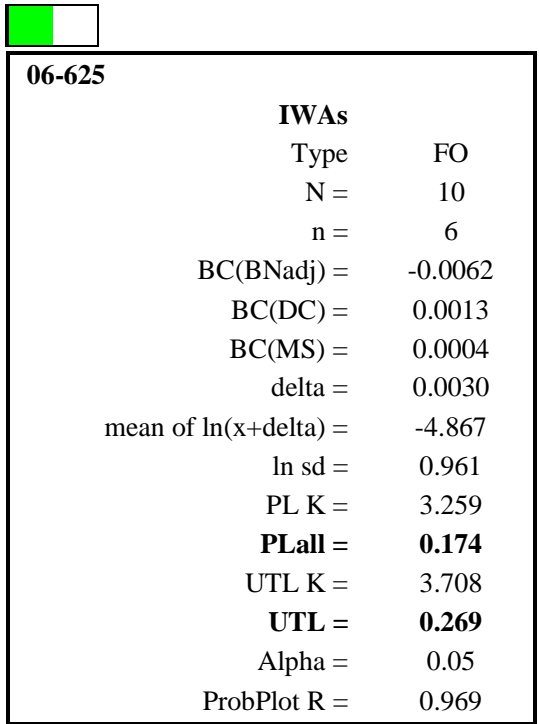

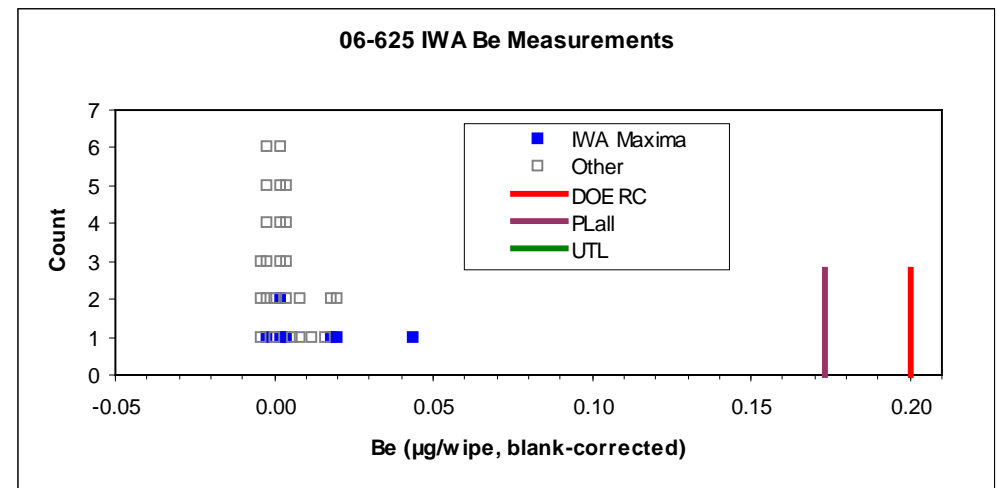

\section{6-800}

Both IWAs were sampled; the maximum value was 0.088 .

\begin{tabular}{|c|c|}
\hline \multicolumn{2}{|l|}{ 06-800 } \\
\hline Area & \\
\hline Type & SH \\
\hline $\mathrm{n}=$ & 42 \\
\hline BC $($ BNadj $)=$ & -0.0062 \\
\hline $\mathrm{BC}(\mathrm{DC})=$ & 0.0013 \\
\hline $\mathrm{BC}(\mathrm{MS})=$ & 0.0004 \\
\hline delta $=$ & 0.0085 \\
\hline mean of $\ln (x+$ delta $)=$ & -2.299 \\
\hline $\ln \mathrm{sd}=$ & 1.380 \\
\hline UTL K = & 2.111 \\
\hline UTL = & 1.843 \\
\hline Alpha $=$ & 0.05 \\
\hline ProbPlot $\mathrm{R}=$ & 0.959 \\
\hline
\end{tabular}

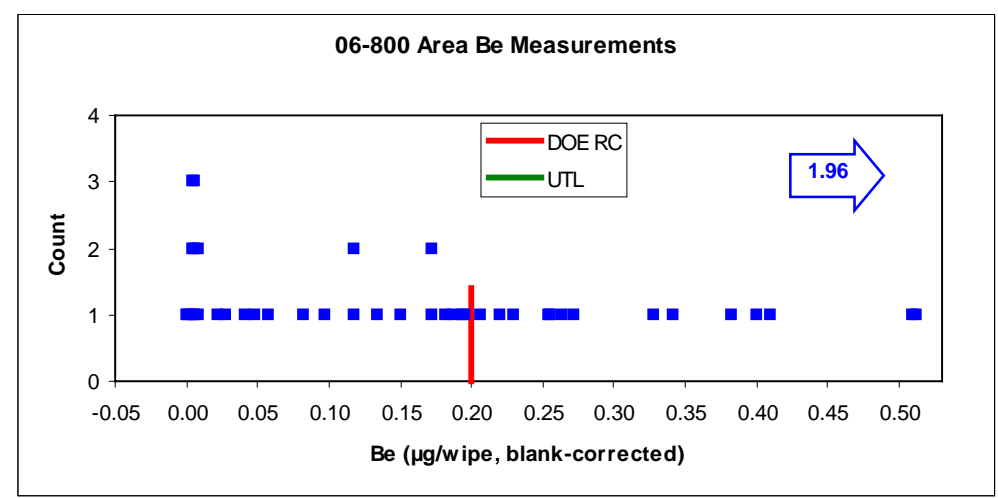

Numerous MS_new measurements exceed the DOE RC; the maximum is $1.96 \mu \mathrm{g} /$ wipe. The Be concentrations and metal ratios in the bulk samples obtained inside the facility are consistent with exterior soil. Most of the metal ratios obtained from the wipe samples with Be $>$ DOE RC are also consistent with exterior soil ratios; the exception is a wipe sample obtained on a piece of equipment, which had Be relatively high compared with $\mathrm{Y}, \mathrm{Nb}$, and $\mathrm{U}$. 


\section{6-900}

\begin{tabular}{rcc|}
\hline & & \\
IWAs & \\
Type & OF \\
$\mathrm{N}=$ & 137 \\
$\mathrm{n}=$ & 29 \\
BC(DC) $=$ & 0.0003 \\
delta $=$ & 0.005 \\
mean of $\ln (\mathrm{x}+$ delta $)$ & $=$ & -4.653 \\
$\ln \mathrm{sd}=$ & 0.225 \\
PL K $=$ & 3.699 \\
PLall $=$ & $\mathbf{0 . 0 1 7}$ \\
UTL K $=$ & 2.232 \\
UTL $=$ & $\mathbf{0 . 0 1 1}$ \\
Alpha $=$ & 0.05 \\
ProbPlot $\mathrm{R}=$ & 0.989 \\
\hline
\end{tabular}

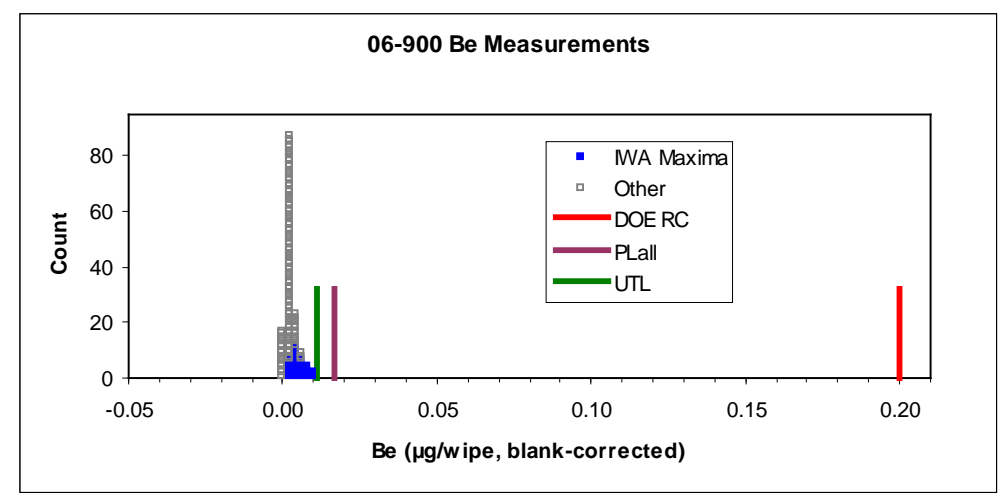

\section{6-901}

\begin{tabular}{|c|c|c|}
\hline \multirow{2}{*}{\multicolumn{3}{|c|}{ 06-901 }} \\
\hline & & \\
\hline & Type & FO \\
\hline & $\mathrm{N}=$ & 10 \\
\hline & $\mathrm{n}=$ & 5 \\
\hline & $\mathrm{BC}(\mathrm{BNadj})=$ & -0.0062 \\
\hline & BC(MS_arch) = & 0.0004 \\
\hline & delta $=$ & 0.0030 \\
\hline & mean of $\ln (x+$ delta $)=$ & -3.513 \\
\hline & $\ln \mathrm{sd}=$ & 1.291 \\
\hline & PL K = & 2.857 \\
\hline & PLall = & 1.187 \\
\hline & UTL K = & 3.400 \\
\hline & $\mathbf{U T L}=$ & 2.395 \\
\hline & Alpha = & 0.05 \\
\hline & ProbPlot R = & 0.930 \\
\hline \multicolumn{3}{|c|}{ UTL cluster sampling analysis } \\
\hline & $\mathrm{F}=$ & 0.98 \\
\hline & delta $=$ & 0.0090 \\
\hline & mean of $\ln (x+$ delta $)=$ & -4.288 \\
\hline & $\ln s d=$ & 1.044 \\
\hline & UTL K = & 2.232 \\
\hline & UTL $=$ & 0.132 \\
\hline & ProbPlot $\mathrm{R}=$ & 0.954 \\
\hline
\end{tabular}

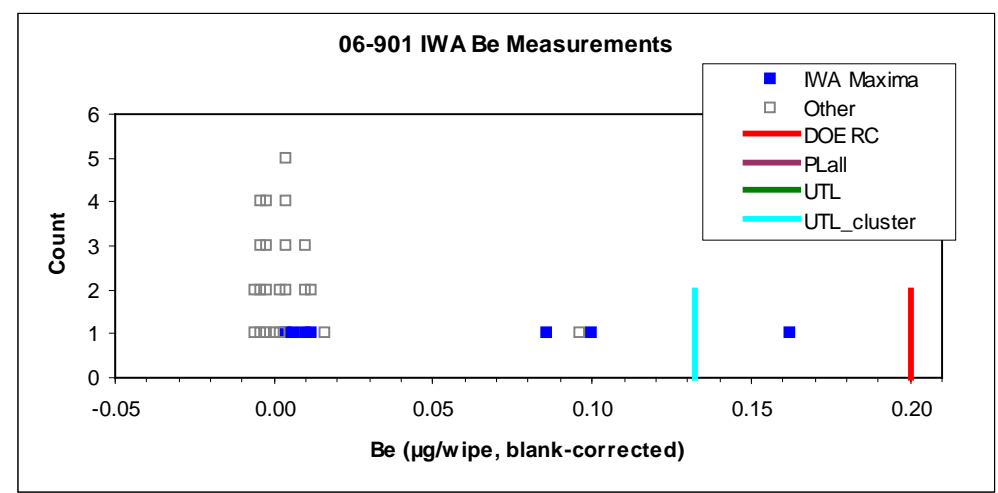




\section{6-902}

\begin{tabular}{|c|c|c|}
\hline \multirow{2}{*}{\multicolumn{3}{|c|}{ 06-902 }} \\
\hline & & \\
\hline & Type & OF \\
\hline & $\mathrm{N}=$ & 39 \\
\hline & $\mathrm{n}=$ & 10 \\
\hline & $\mathrm{BC}(\mathrm{BNadj})=$ & -0.0062 \\
\hline & $\mathrm{BC}(\mathrm{DC})=$ & 0.0013 \\
\hline & BC(MS_arch) $=$ & 0.0004 \\
\hline & BC(MS_new) = & 0.0001 \\
\hline & delta $=$ & 0.0030 \\
\hline & mean of $\ln (x+$ delta $)=$ & -4.191 \\
\hline & $\ln \mathrm{sd}=$ & 1.218 \\
\hline & $\mathrm{PL} K=$ & 3.871 \\
\hline & PLall = & 1.684 \\
\hline & UTL K = & 2.911 \\
\hline & UTL = & 0.521 \\
\hline & Alpha = & 0.05 \\
\hline & ProbPlot $\mathrm{R}=$ & 0.931 \\
\hline \multicolumn{3}{|c|}{ omitting door tops } \\
\hline & mean of $\ln (x+$ delta $)=$ & -4.754 \\
\hline & $\ln \mathrm{sd}=$ & 0.735 \\
\hline & PLall = & 0.145 \\
\hline & UTL $=$ & 0.070 \\
\hline & ProbPlot $\mathrm{R}=$ & 0.897 \\
\hline
\end{tabular}

\section{6-904}

\begin{tabular}{|c|c|}
\hline \multirow{2}{*}{\multicolumn{2}{|c|}{ 06-904 }} \\
\hline & \\
\hline Type & $\mathrm{SH}$ \\
\hline $\mathrm{N}=$ & 34 \\
\hline $\mathrm{n}=$ & 17 \\
\hline $\mathrm{BC}(\mathrm{BNadj})=$ & -0.0062 \\
\hline $\mathrm{BC}(\mathrm{DC})=$ & 0.0013 \\
\hline BC(MS_arch) = & 0.0004 \\
\hline delta $=$ & 0.0030 \\
\hline mean of $\ln (x+$ delta $)=$ & -5.059 \\
\hline $\ln \mathrm{sd}=$ & 0.532 \\
\hline PL K = & 3.314 \\
\hline PLall $=$ & 0.034 \\
\hline UTL K = & 2.553 \\
\hline UTL = & 0.022 \\
\hline Alpha $=$ & 0.04 \\
\hline ProbPlot $\mathrm{R}=$ & 0.988 \\
\hline
\end{tabular}

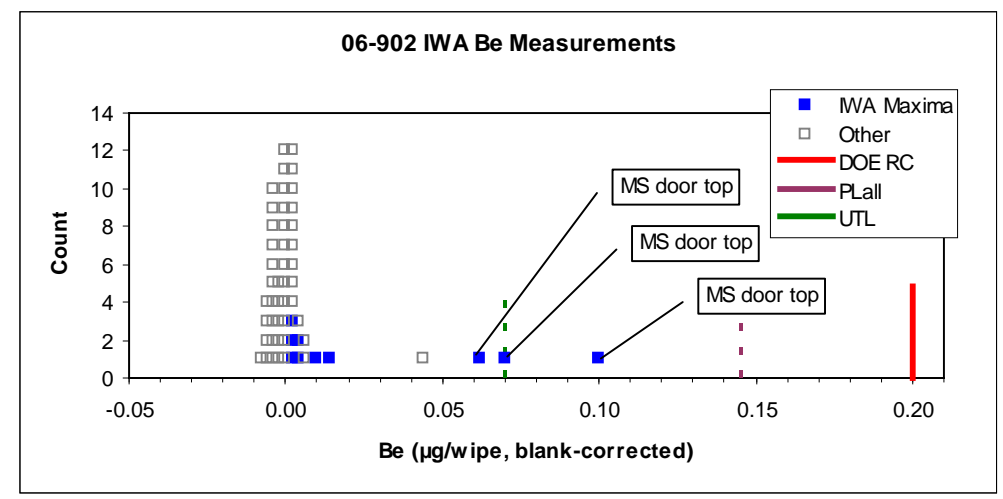

There are three outliers in the MS_arch data obtained from door tops. All are less than the DOE RC, but push both PLall and UTL over it. Both PLall and UTL are less than RC if those locations are omitted.

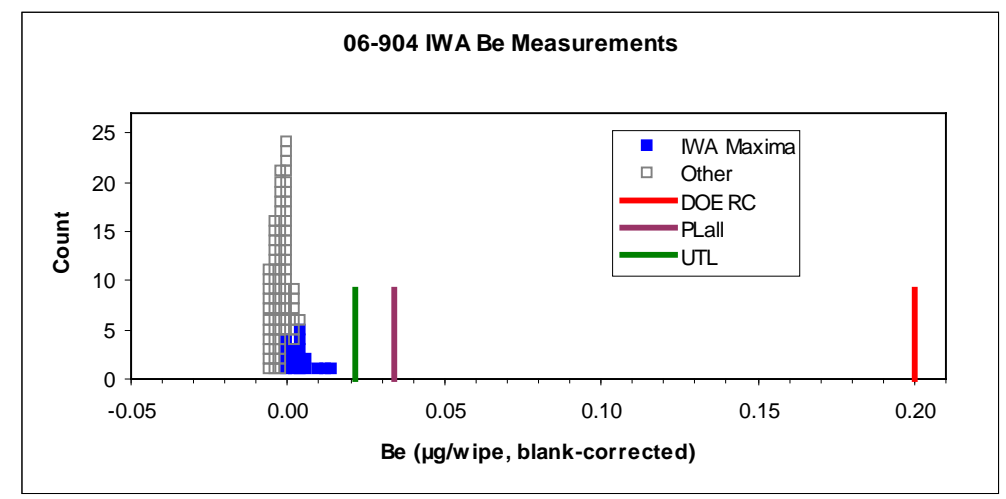




\begin{tabular}{|c|c|}
\hline \multicolumn{2}{|l|}{ 06-904 } \\
\hline Area & \\
\hline Type & SH \\
\hline $\mathrm{n}=$ & 62 \\
\hline $\mathrm{BC}(\mathrm{BNadj})=$ & -0.0062 \\
\hline $\mathrm{BC}(\mathrm{DC})=$ & 0.0013 \\
\hline BC(MS_arch $)=$ & 0.0004 \\
\hline delta $=$ & 0.0080 \\
\hline mean of $\ln (x+$ delta $)=$ & -4.483 \\
\hline $\ln \mathrm{sd}=$ & 0.952 \\
\hline UTL K = & 2.191 \\
\hline UTL $=$ & 0.083 \\
\hline Alpha $=$ & 0.01 \\
\hline ProbPlot $\mathrm{R}=$ & 0.979 \\
\hline
\end{tabular}

\section{6-906}

\begin{tabular}{|c|c|}
\hline \multicolumn{2}{|l|}{$06-906$} \\
\hline \multicolumn{2}{|l|}{ IWAs } \\
\hline Type & $\mathrm{SH}$ \\
\hline $\mathrm{N}=$ & 11 \\
\hline $\mathrm{n}=$ & 6 \\
\hline $\mathrm{BC}(\mathrm{BNadj})=$ & -0.0062 \\
\hline $\mathrm{BC}(\mathrm{DC})=$ & 0.0013 \\
\hline BC(MS_arch) $=$ & 0.0004 \\
\hline delta $=$ & 0.0030 \\
\hline mean of $\ln (x+$ delta $)=$ & -3.827 \\
\hline $\ln \mathrm{sd}=$ & 1.426 \\
\hline PL K = & 3.659 \\
\hline PLall $=$ & 4.020 \\
\hline UTL K = & 3.918 \\
\hline UTL $=$ & 5.821 \\
\hline Alpha $=$ & 0.04 \\
\hline ProbPlot $\mathrm{R}=$ & 0.975 \\
\hline \multicolumn{2}{|l|}{ omitting door tops } \\
\hline mean of $\ln (x+$ delta $)=$ & -4.853 \\
\hline $\ln \mathrm{sd}=$ & 0.959 \\
\hline PLall $=$ & 0.257 \\
\hline $\mathbf{U T L}=$ & 0.331 \\
\hline ProbPlot $\mathrm{R}=$ & 0.965 \\
\hline \multicolumn{2}{|c|}{ UTL cluster analysis, all data } \\
\hline $\mathrm{F}=$ & 0.65 \\
\hline delta $=$ & 0.0090 \\
\hline mean of $\ln (x+$ delta $)=$ & -4.436 \\
\hline $\ln \mathrm{sd}=$ & 0.683 \\
\hline UTL K = & 2.336 \\
\hline UTL_cluster = & 0.049 \\
\hline Alpha $=$ & 0.025 \\
\hline ProbPlot $\mathrm{R}=$ & 0.814 \\
\hline
\end{tabular}
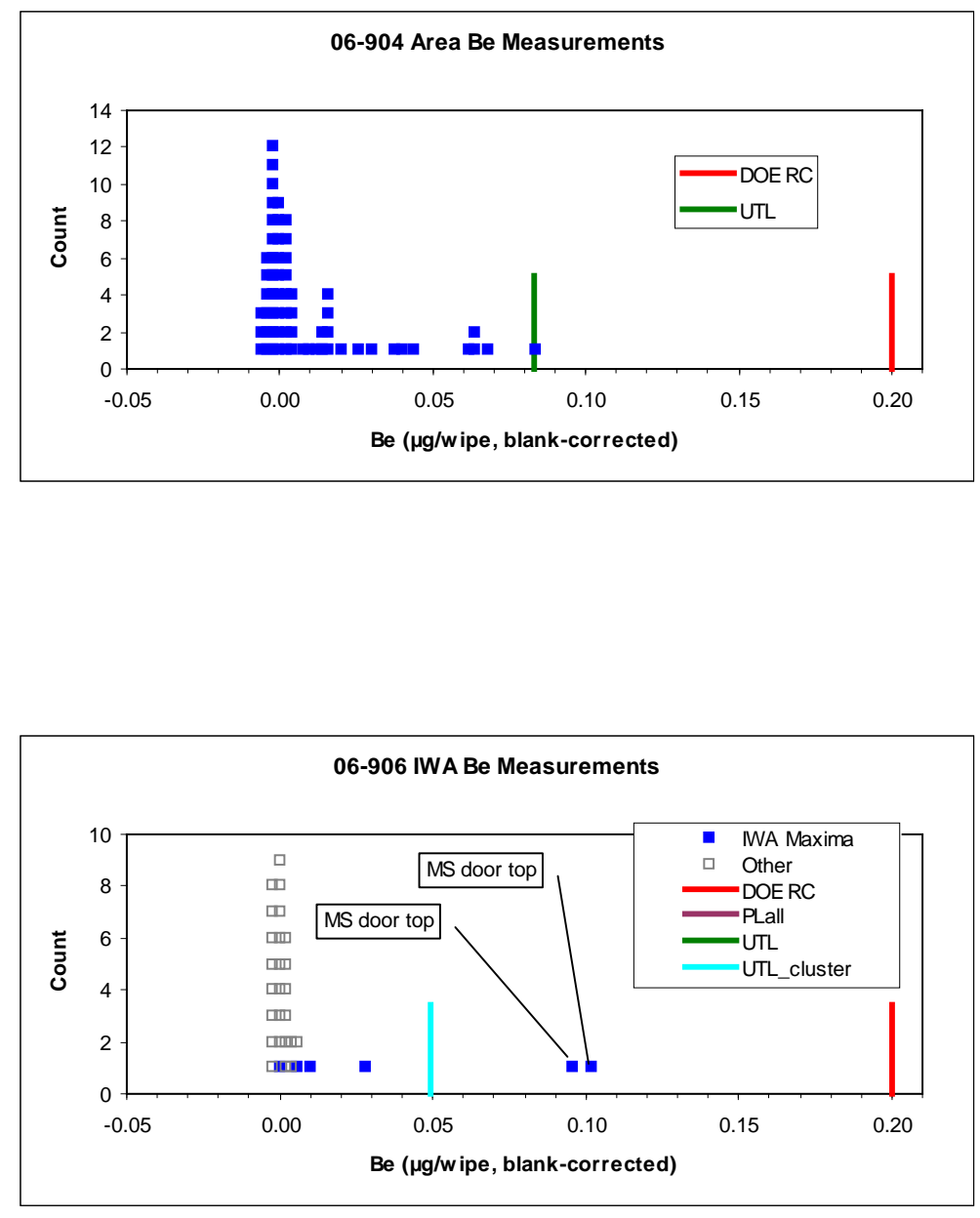

The two high values (still considerably less than the DOE RC) are MS_arch analyses from door tops. Omitting these locations does not pull PLall or UTL down quite far enough due the small number of IWAs involved. UTL_cluster is adequately low; the low ProbPlot $\mathrm{R}$ is due to those outliers. 

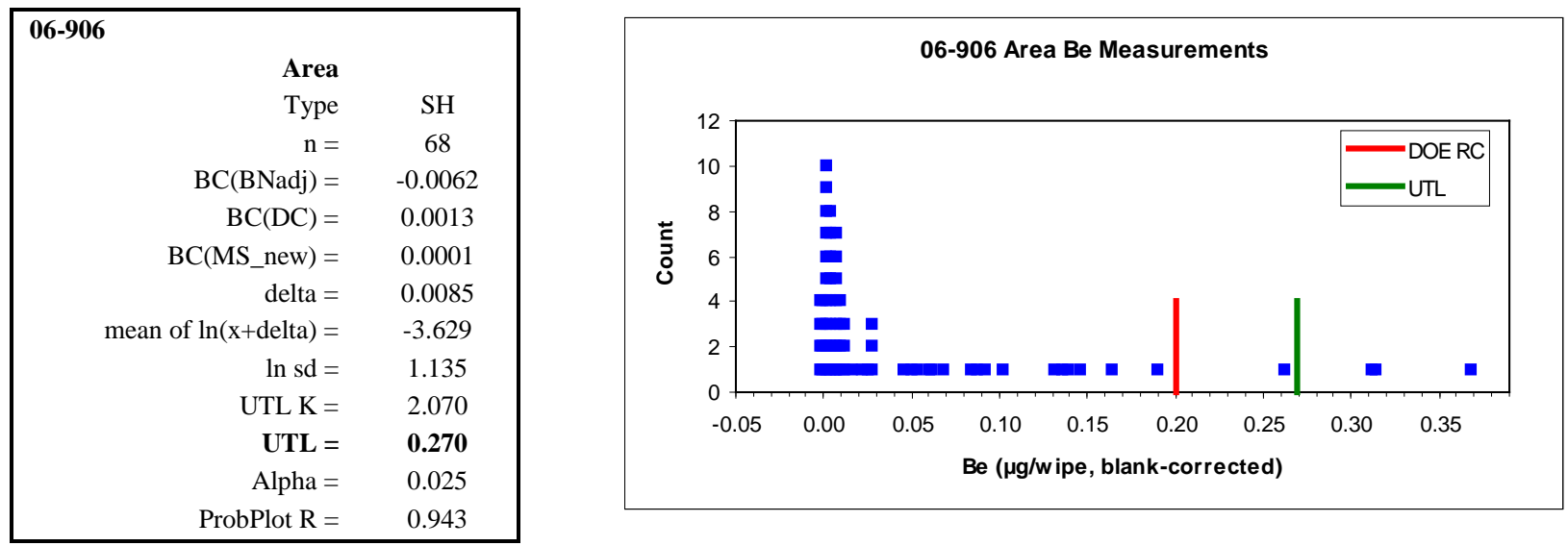

In the Area portion of the facility there are four MS_new results above the DOE RC; UTL also exceeds the DOE RC. The Be concentrations and metal ratios in the four bulk samples obtained inside the facility are consistent with exterior soil samples at the NTS. Most of the metal ratios obtained from the four high wipe samples are likewise consistent with the exterior soils; the exceptions are to $\mathrm{Ni} / \mathrm{Be}$ ratios. Recall, though, that $\mathrm{Ni} / \mathrm{Be}$ is the least reliable of the six metal ratios for these comparisons with exterior soils.

\section{6-908}

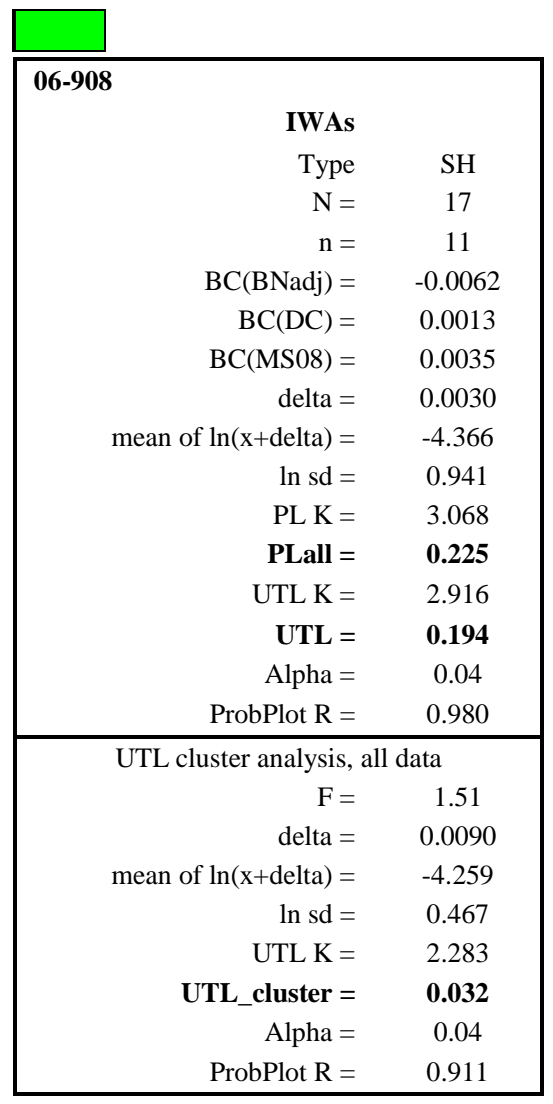

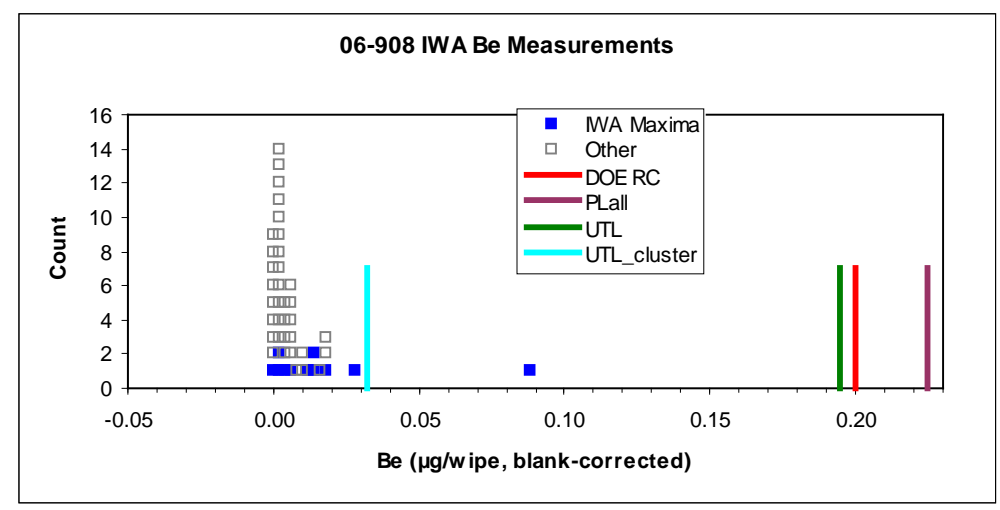

For this facility UTL is slightly less than the DOE RC; it is inflated by the one relatively high value, which came from the top of a milling machine. The UTL_cluster, which takes into account all the data rather than just the IWA maxima, is much lower. 


\begin{tabular}{|c|c|}
\hline \multicolumn{2}{|l|}{ 06-908 } \\
\hline Area & \\
\hline Type & SH \\
\hline $\mathrm{n}=$ & 62 \\
\hline $\mathrm{BC}(\mathrm{BNadj})=$ & -0.0062 \\
\hline $\mathrm{BC}(\mathrm{DC})=$ & 0.0013 \\
\hline $\mathrm{BC}(\mathrm{MS})=$ & 0.0004 \\
\hline delta $=$ & 0.0085 \\
\hline mean of $\ln (x+$ delta $)=$ & -4.070 \\
\hline $\ln \mathrm{sd}=$ & 0.527 \\
\hline UTL K = & 2.191 \\
\hline $\mathbf{U T L}=$ & 0.046 \\
\hline Alpha $=$ & 0.01 \\
\hline ProbPlot $\mathrm{R}=$ & 0.991 \\
\hline
\end{tabular}

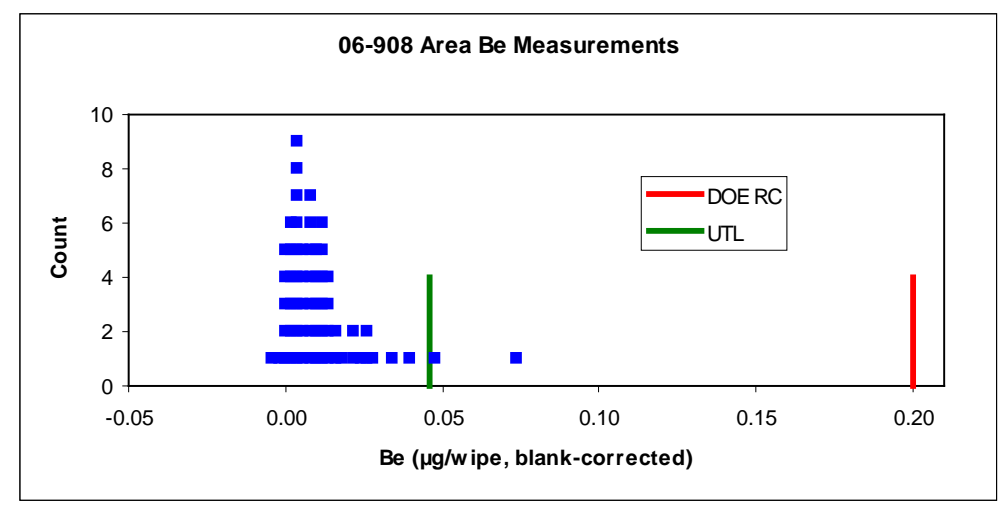

\section{6-914}

\section{$\square$}

06-914

\begin{tabular}{rc|} 
IWAs & \\
Type & $\mathrm{SH}$ \\
$\mathrm{N}=$ & 11 \\
$\mathrm{n}=$ & 6 \\
$\mathrm{BC}(\mathrm{BNadj})=$ & -0.0062 \\
$\mathrm{BC}(\mathrm{DC})=$ & 0.0013 \\
$\mathrm{BC}(\mathrm{MS}$ arch $)=$ & 0.0004 \\
delta $=$ & 0.0030 \\
mean of $\ln (\mathrm{x}+$ delta $)=$ & -3.819 \\
$\ln \mathrm{sd}=$ & 1.140 \\
$\mathrm{PL} \mathrm{K}=$ & 3.659 \\
PLall $=$ & $\mathbf{1 . 4 2 1}$ \\
UTL K $=$ & 3.918 \\
UTL $=$ & $\mathbf{1 . 9 1 1}$ \\
Alpha $=$ & 0.04 \\
ProbPlot $\mathrm{R}=$ & 0.925 \\
\hline
\end{tabular}

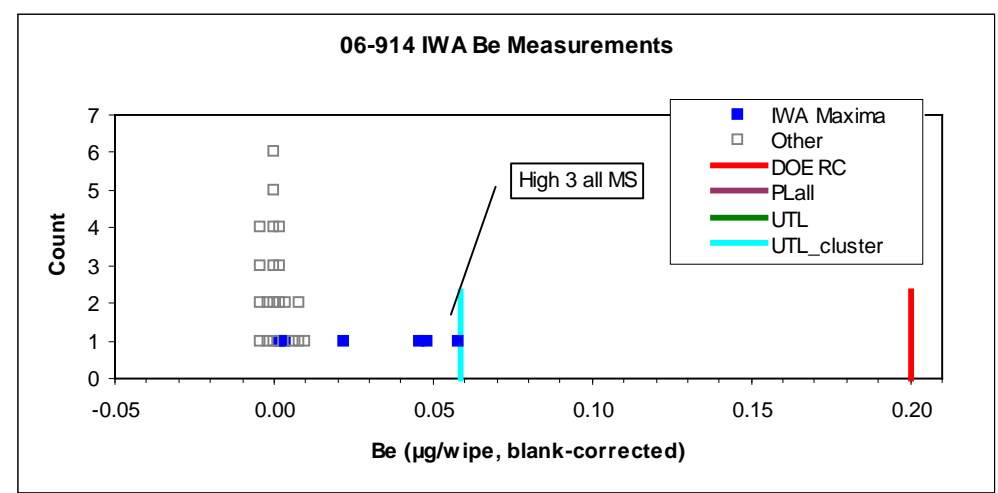

The three relatively high values are all MS_arch.

UTL cluster sampling analysis

$\mathrm{F}=\quad 0.84$

delta $=\quad 0.0090$

mean of $\ln (\mathrm{x}+$ delta $)=\quad-4.371$

$\ln \mathrm{sd}=\quad 0.694$

UTL K $=\quad 2.421$

UTL $=\quad 0.059$

Alpha $=\quad 0.025$

ProbPlot $\mathrm{R}=\quad 0.926$ 


\begin{tabular}{|c|c|}
\hline \multirow{2}{*}{\multicolumn{2}{|c|}{ 06-914 }} \\
\hline & \\
\hline Туре & SH \\
\hline $\mathrm{n}=$ & 58 \\
\hline $\mathrm{BC}(\mathrm{BNadj})=$ & -0.0062 \\
\hline $\mathrm{BC}(\mathrm{DC})=$ & 0.0013 \\
\hline BC(MS_new) $=$ & 0.0001 \\
\hline delta $=$ & 0.0085 \\
\hline mean of $\ln (x+$ delta $)=$ & -3.603 \\
\hline $\ln \mathrm{sd}=$ & 1.125 \\
\hline UTL K = & 2.113 \\
\hline UTL = & 0.285 \\
\hline Alpha = & 0.025 \\
\hline ProbPlot $\mathrm{R}=$ & 0.962 \\
\hline
\end{tabular}

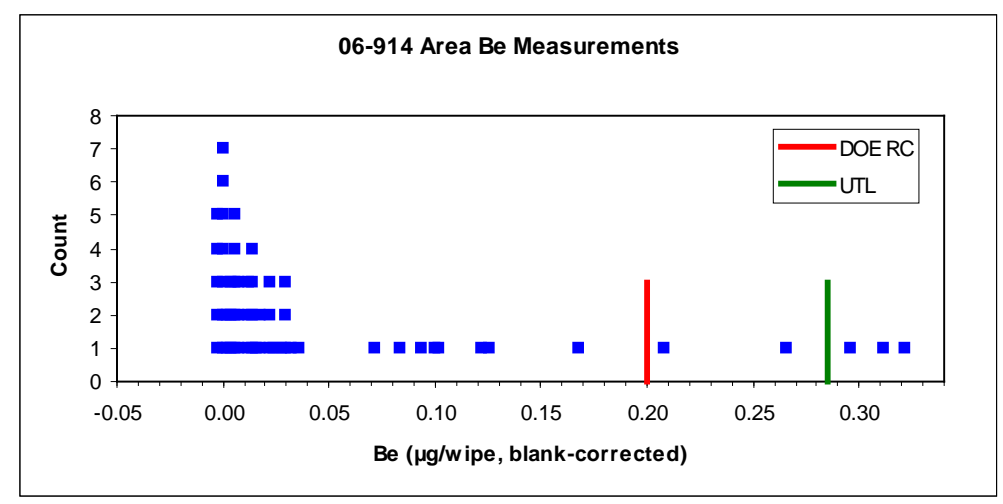

The Area UTL and five MS_new measurements exceed the DOE RC in the Area portion of this facility. The Be concentrations and metal ratios in both bulk samples are consistent with exterior soils at the NTS. The metal ratios of the high wipes are either consistent with exterior soils or reflect somewhat relatively low Be concentrations compared with the other metals.

\section{6-CP1}

\begin{tabular}{|rc|}
\hline & \\
\hline 06-CP1 & \\
IWAs & \\
Type & OF \\
$\mathrm{N}=$ & 107 \\
$\mathrm{n}=$ & 19 \\
BC(DC) $=$ & 0.0003 \\
delta $=$ & 0.0050 \\
ln sd $=$ & 0.517 \\
PL K $=$ & 3.837 \\
PLall $=$ & $\mathbf{0 . 0 7 3}$ \\
UTL K $=$ & 2.423 \\
UTL $=$ & $\mathbf{0 . 0 3 3}$ \\
Alpha $=$ & 0.05 \\
ProbPlot R $=$ & 0.966 \\
\hline
\end{tabular}

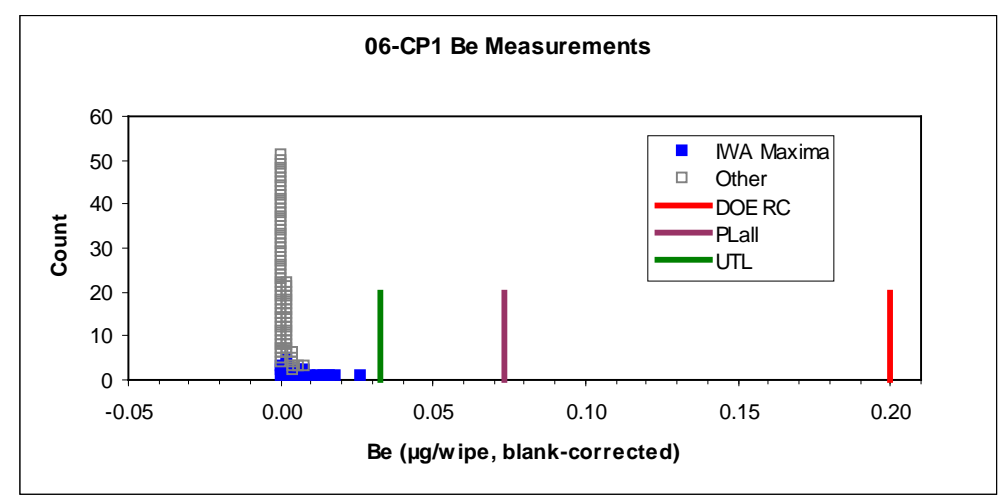




\section{6-CP100}

\section{$\square$}

06-CP100

$\begin{array}{rc}\text { IWAs } & \\ \text { Type } & \mathrm{ST} \\ \mathrm{N}= & 11 \\ \mathrm{n}= & 6 \\ \mathrm{BC}(\mathrm{BNadj})= & -0.0062 \\ \mathrm{BC}(\mathrm{DC})= & 0.0013 \\ \text { delta }= & 0.0030 \\ \text { mean of } \ln (\mathrm{x}+\text { delta })= & -5.161 \\ \ln \mathrm{sd}= & 0.551 \\ \text { PL K }= & 3.659 \\ \text { PLall }= & \mathbf{0 . 0 4 0} \\ \mathrm{UTL} \mathrm{K}= & 3.918 \\ \text { UTL }= & \mathbf{0 . 0 4 7} \\ \text { Alpha }= & 0.04 \\ \text { ProbPlot } \mathrm{R}= & 0.791\end{array}$

\begin{tabular}{|c|c|}
\hline \multirow{2}{*}{\multicolumn{2}{|c|}{ 06-CP100 }} \\
\hline & \\
\hline Type & ST \\
\hline $\mathrm{n}=$ & 33 \\
\hline $\mathrm{BC}(\mathrm{BNadj})=$ & -0.0062 \\
\hline $\mathrm{BC}(\mathrm{DC})=$ & 0.0013 \\
\hline $\mathrm{BC}(\mathrm{MS})=$ & 0.0004 \\
\hline delta $=$ & 0.0080 \\
\hline mean of $\ln (x+$ delta $)=$ & -4.795 \\
\hline $\ln \mathrm{sd}=$ & 0.587 \\
\hline UTL K = & 2.461 \\
\hline UTL = & 0.027 \\
\hline Alpha $=$ & 0.01 \\
\hline ProbPlot $\mathrm{R}=$ & 0.967 \\
\hline
\end{tabular}

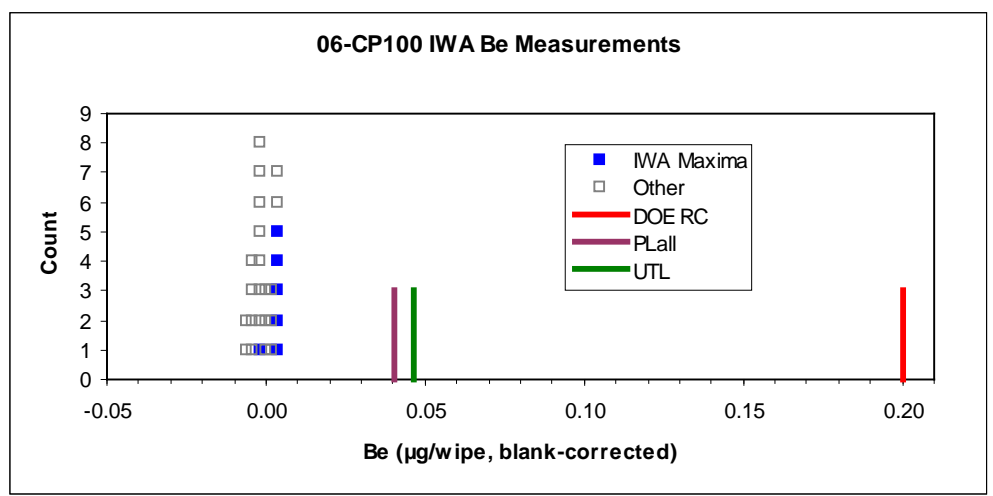

One of the IWA maxima is atypically low, hence the low ProbPlot R.

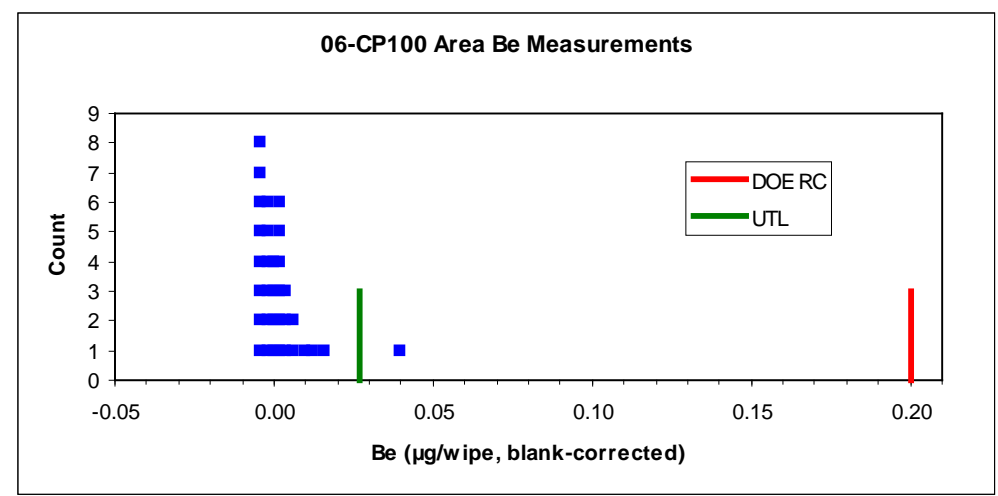




\section{6-CP160}

\section{$\square$}

\begin{tabular}{|c|c|}
\hline \multicolumn{2}{|l|}{ 06-CP160 } \\
\hline Type & $\mathrm{SH}$ \\
\hline $\mathrm{N}=$ & 14 \\
\hline $\mathrm{n}=$ & 6 \\
\hline $\mathrm{BC}(\mathrm{BNadj})=$ & -0.0062 \\
\hline $\mathrm{BC}(\mathrm{DC})=$ & 0.0013 \\
\hline BC(MS_arch $)=$ & 0.0004 \\
\hline delta $=$ & 0.003 \\
\hline mean of $\ln (x+$ delta $)=$ & -3.899 \\
\hline $\ln s d=$ & 0.806 \\
\hline PL K = & 4.039 \\
\hline PLall = & 0.523 \\
\hline UTL K = & 3.918 \\
\hline UTL = & 0.474 \\
\hline Alpha = & 0.04 \\
\hline ProbPlot $\mathrm{R}=$ & 0.926 \\
\hline \multicolumn{2}{|c|}{ UTL cluster sampling analysis } \\
\hline $\mathrm{F}=$ & 1.33 \\
\hline delta $=$ & 0.0090 \\
\hline mean of $\ln (x+$ delta $)=$ & -4.363 \\
\hline $\operatorname{tau}^{\wedge} 2=$ & 0.019 \\
\hline $\operatorname{sig} \wedge 2=$ & 0.293 \\
\hline $\ln s d=$ & 0.558 \\
\hline UTL K = & 2.351 \\
\hline UTL = & 0.038 \\
\hline Alpha $=$ & 0.025 \\
\hline ProbPlot $\mathrm{R}=$ & 0.921 \\
\hline
\end{tabular}

\begin{tabular}{|rc|}
\hline 06-CP160 & \\
Area & \\
Type & SH \\
$\mathrm{n}=$ & 25 \\
& -0.0062 \\
BC(BNadj $)=$ & 0.0013 \\
BC(DC) $=$ & 0.0001 \\
BC(MS_new $)=$ & 0.0085 \\
delta $=$ & -3.729 \\
& 1.005 \\
& 2.442 \\
UTL K $=$ & $\mathbf{0 . 2 7 1}$ \\
UTL $=$ & 0.025 \\
Alpha $=$ & 0.977 \\
ProbPlot R $=$ &
\end{tabular}
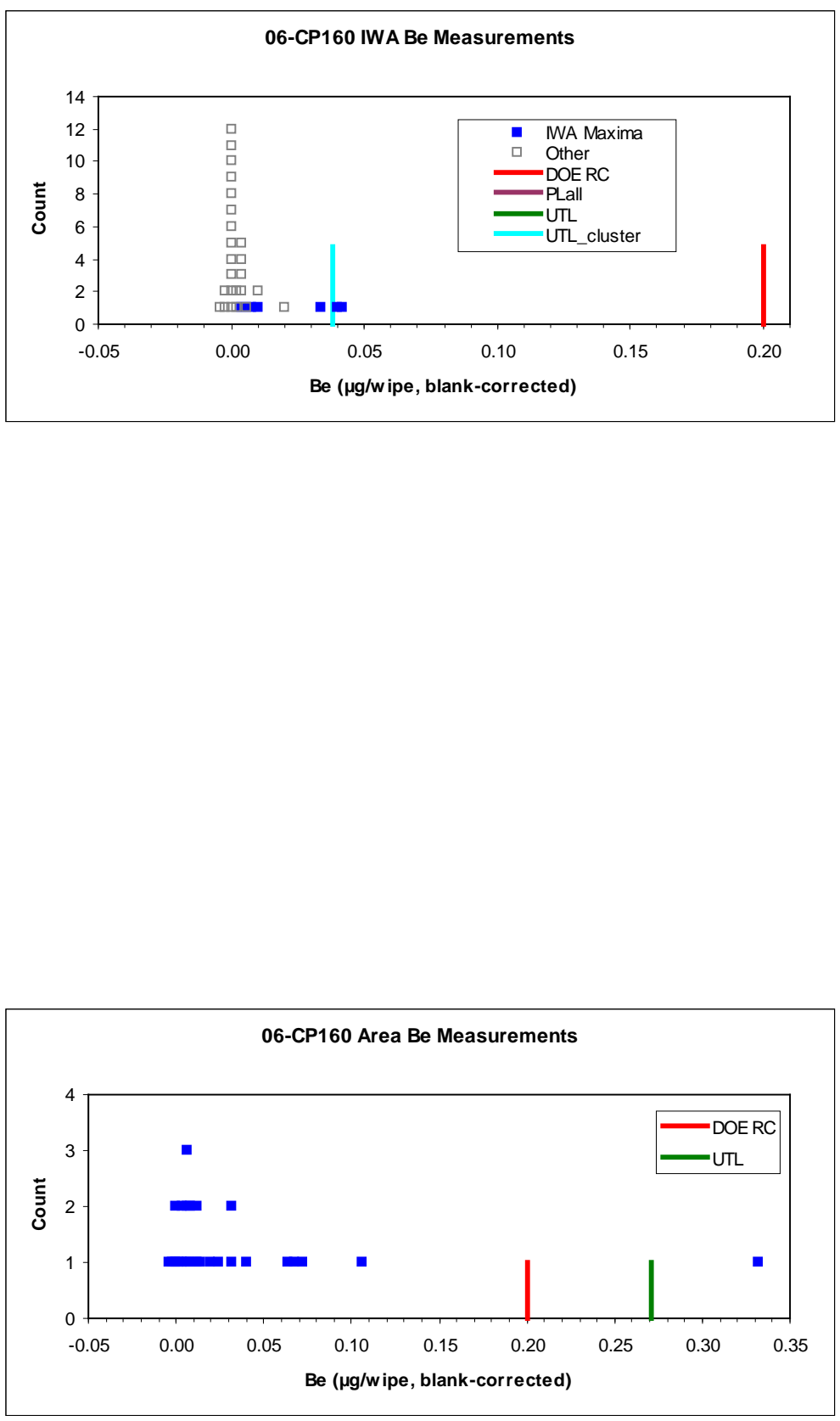

There is an isolated MS_new value above the DOE RC in the Area portion of this facility; it was obtained on the floor of the mezzanine. The Be concentrations on the two bulk samples are rather low. The metal ratios in the high wipe sample are consistent with those in NTS exterior soils. 


\section{6-CP161}

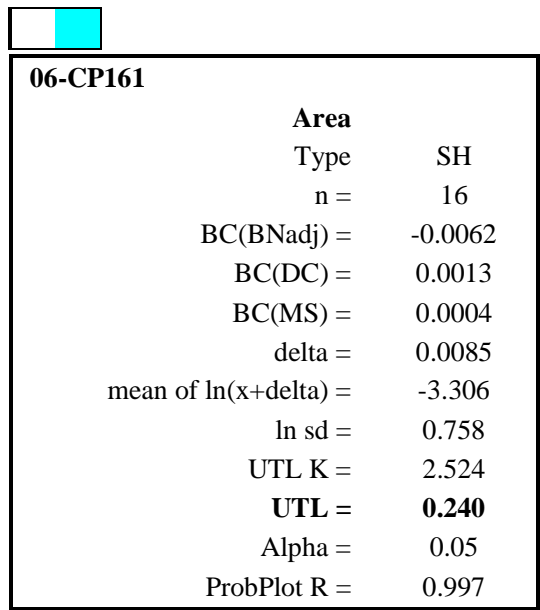

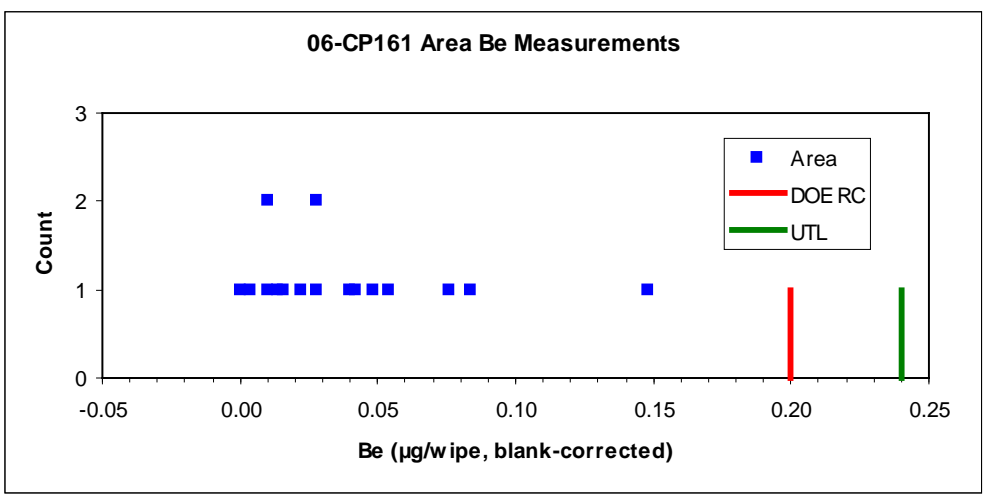

The Be concentration in the bulk sample obtained inside this facility is on the low side; the metal ratios are consistent with NTS exterior soils.

\section{6-CP162}

\begin{tabular}{|c|c|}
\hline \multicolumn{2}{|l|}{ 06-CP162 } \\
\hline \multicolumn{2}{|l|}{ IWAs } \\
\hline Туре & $\mathrm{SH}$ \\
\hline $\mathrm{N}=$ & 8 \\
\hline $\mathrm{n}=$ & 4 \\
\hline $\mathrm{BC}(\mathrm{BNadj})=$ & -0.0062 \\
\hline $\mathrm{BC}(\mathrm{DC})=$ & 0.0013 \\
\hline BC(MS_arch $)=$ & 0.0004 \\
\hline delta $=$ & 0.0030 \\
\hline mean of $\ln (x+$ delta $)=$ & -3.432 \\
\hline $\ln \mathrm{sd}=$ & 1.599 \\
\hline PL K = & 4.593 \\
\hline PLall = & 49.958 \\
\hline UTL K = & 5.580 \\
\hline UTL $=$ & 242.282 \\
\hline Alpha $=$ & 0.04 \\
\hline ProbPlot $\mathrm{R}=$ & 0.997 \\
\hline \multicolumn{2}{|l|}{ omitting door top } \\
\hline mean of $\ln (x+$ delta $)=$ & -3.990 \\
\hline $\ln \mathrm{sd}=$ & 0.945 \\
\hline PLall $=$ & 1.416 \\
\hline UTL $=$ & 3.604 \\
\hline ProbPlot $\mathrm{R}=$ & 0.993 \\
\hline \multicolumn{2}{|l|}{$\begin{array}{l}\text { UTL cluster analysis } \\
\text { omitting door top }\end{array}$} \\
\hline $\mathrm{F}=$ & 0.77 \\
\hline delta $=$ & 0.0090 \\
\hline mean of $\ln (x+$ delta $)=$ & -4.474 \\
\hline $\ln \mathrm{sd}=$ & 0.693 \\
\hline UTL K = & 2.693 \\
\hline UTL = & 0.065 \\
\hline Alpha $=$ & 0.025 \\
\hline ProbPlot $\mathrm{R}=$ & 0.980 \\
\hline
\end{tabular}

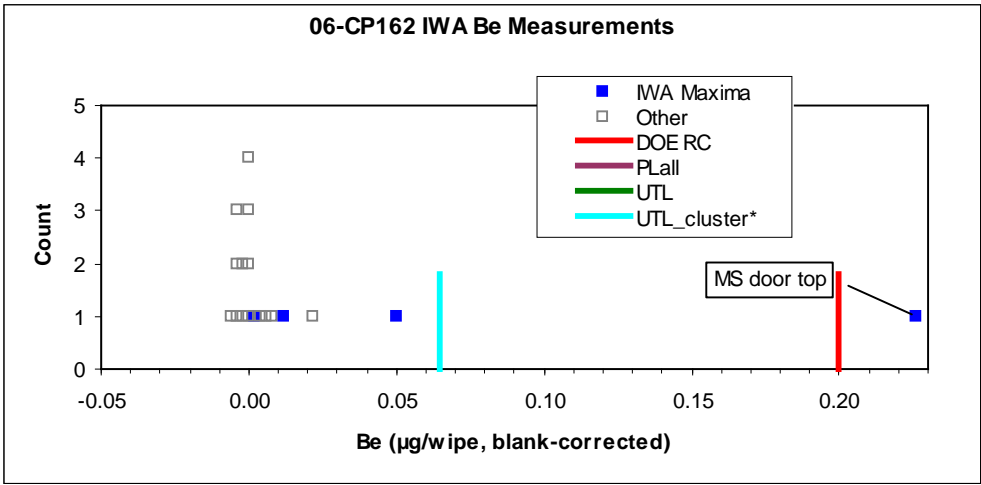

There is one high MS_arch measurement obtained on a door top; otherwise there are few IWAs involved. Omitting the door top sample gives UTL_cluster less than the DOE RC. 


\begin{tabular}{|c|c|}
\hline \multirow{2}{*}{\multicolumn{2}{|c|}{ 06-CР162 }} \\
\hline & \\
\hline Type & SH \\
\hline $\mathrm{n}=$ & 24 \\
\hline $\mathrm{BC}(\mathrm{BNadj})=$ & -0.0062 \\
\hline $\mathrm{BC}(\mathrm{DC})=$ & 0.0013 \\
\hline BC(MS_new) $=$ & 0.0001 \\
\hline delta $=$ & 0.0085 \\
\hline mean of $\ln (x+$ delta $)=$ & -3.621 \\
\hline $\ln \mathrm{sd}=$ & 1.066 \\
\hline UTL K = & 2.465 \\
\hline UTL = & 0.362 \\
\hline Alpha $=$ & 0.025 \\
\hline ProbPlot $\mathrm{R}=$ & 0.922 \\
\hline
\end{tabular}

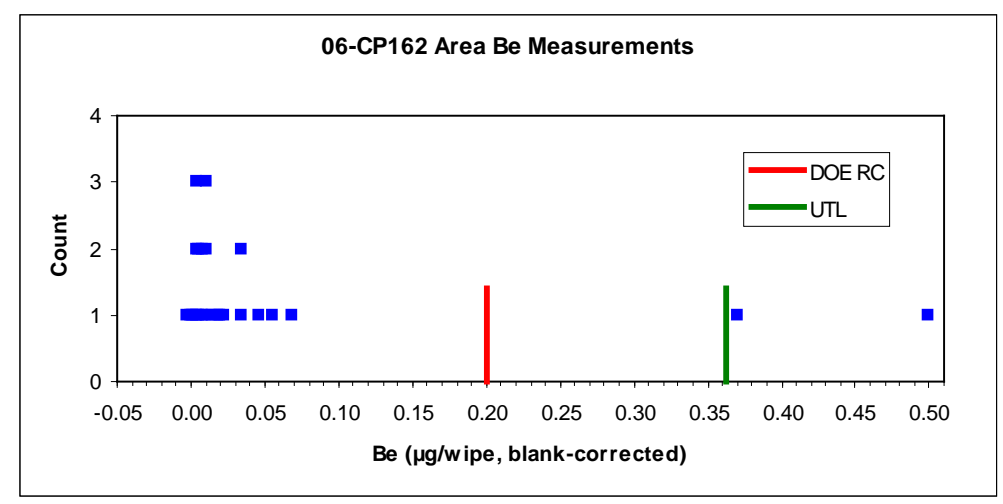

There are two isolated high MS_new values, both obtained near a shop door. The Be concentration and metal ratios from the bulk sample obtained inside the facility are consistent with NTS exterior soils, as are the metal ratios in the high wipes.

\section{6-CP213}

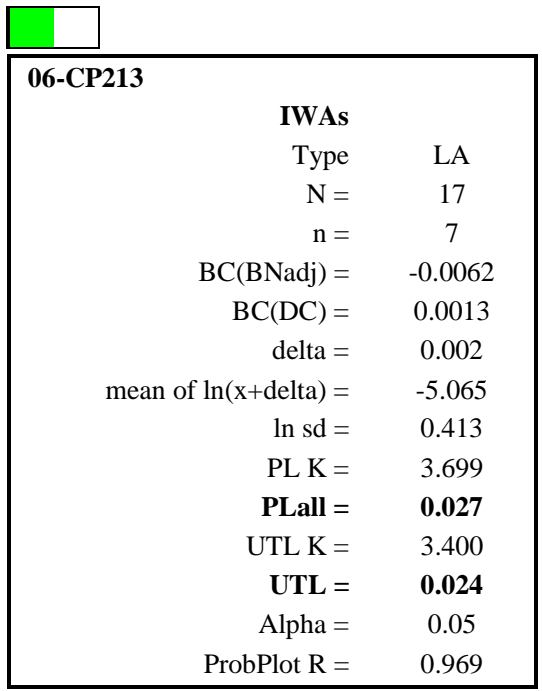

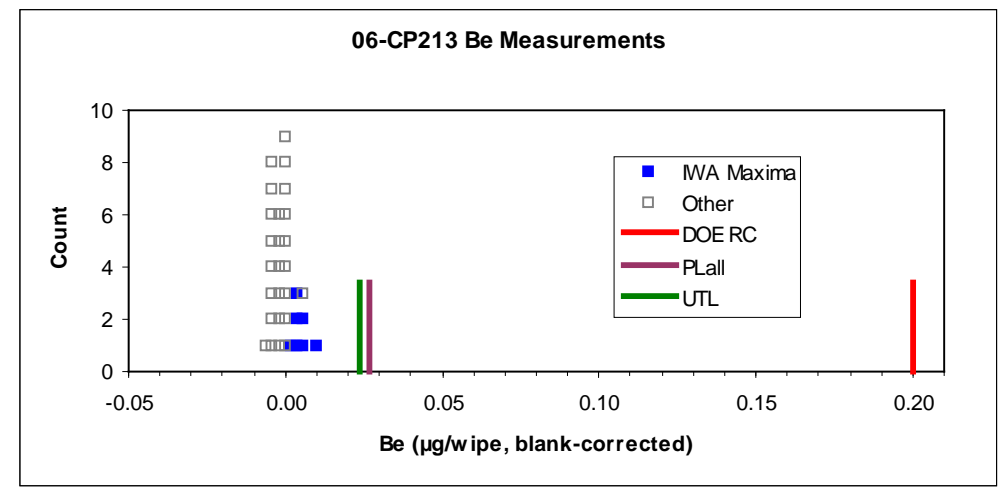




\section{6-CP214}

\begin{tabular}{|c|c|}
\hline \multicolumn{2}{|l|}{ 06-CР214 } \\
\hline IWAs & \\
\hline Type & OF \\
\hline $\mathrm{N}=$ & 13 \\
\hline $\mathrm{n}=$ & 7 \\
\hline $\mathrm{BC}(\mathrm{BNadj})=$ & -0.0062 \\
\hline $\mathrm{BC}(\mathrm{DC})=$ & 0.0013 \\
\hline delta $=$ & 0.0050 \\
\hline mean of $\ln (x+$ delta $)=$ & -4.574 \\
\hline $\ln \mathrm{sd}=$ & 0.450 \\
\hline PL K = & 3.345 \\
\hline PLall $=$ & 0.041 \\
\hline UTL K = & 3.400 \\
\hline UTL = & 0.043 \\
\hline Alpha $=$ & 0.05 \\
\hline ProbPlot $\mathrm{R}=$ & 0.949 \\
\hline
\end{tabular}

\section{6-CP215}

\begin{tabular}{|c|c|}
\hline \multicolumn{2}{|l|}{ 06-СР215 } \\
\hline IWAs & \\
\hline Type & $\mathrm{SH}$ \\
\hline $\mathrm{N}=$ & 11 \\
\hline $\mathrm{n}=$ & 7 \\
\hline $\mathrm{BC}(\mathrm{BNadj})=$ & -0.0062 \\
\hline $\mathrm{BC}(\mathrm{DC})=$ & 0.0013 \\
\hline BC(MS_arch) $=$ & 0.0004 \\
\hline delta $=$ & 0.003 \\
\hline mean of $\ln (x+$ delta $)=$ & -4.432 \\
\hline $\ln \mathrm{sd}=$ & 1.446 \\
\hline PL K = & 3.250 \\
\hline PLall = & 1.300 \\
\hline UTL K = & 3.569 \\
\hline UTL = & 2.066 \\
\hline Alpha $=$ & 0.04 \\
\hline ProbPlot $\mathrm{R}=$ & 0.931 \\
\hline \multicolumn{2}{|c|}{ UTL cluster sampling analysis } \\
\hline $\mathrm{F}=$ & 5.71 \\
\hline delta $=$ & 0.0090 \\
\hline mean of $\ln (x+$ delta $)=$ & -4.749 \\
\hline $\operatorname{tau}^{\wedge} 2=$ & 0.501 \\
\hline $\operatorname{sig} \wedge 2=$ & 0.425 \\
\hline $\ln \mathrm{sd}=$ & 0.962 \\
\hline UTL K = & 3.096 \\
\hline UTL = & 0.161 \\
\hline Alpha $=$ & 0.025 \\
\hline ProbPlot $\mathrm{R}=$ & 0.944 \\
\hline
\end{tabular}
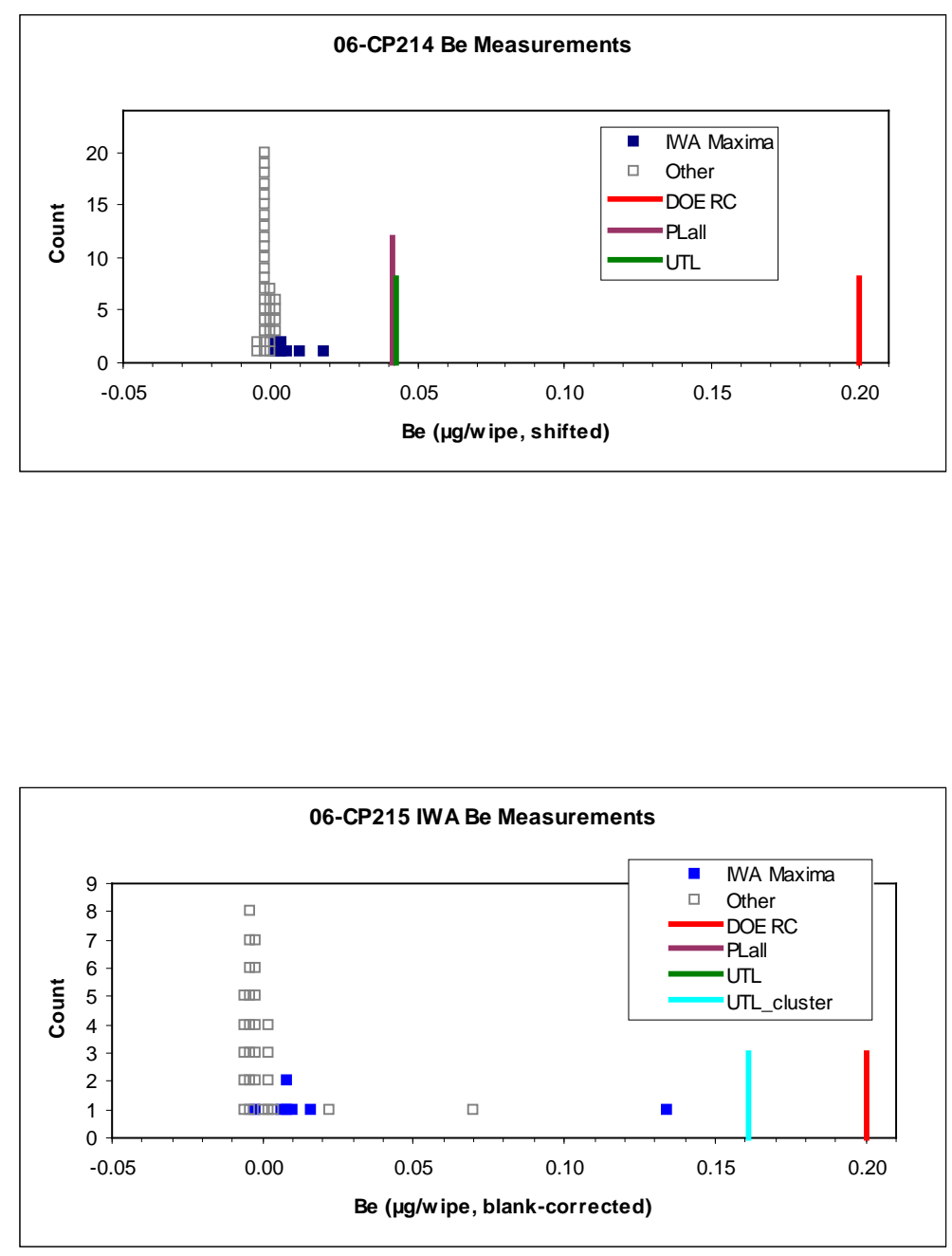

The two high values are from Room 107; the highest is MS_arch. In this UTL cluster sampling analysis there is a distinct difference in levels among the IWAs sampled, as reflected in the elevated $\mathrm{F}$ statistic. 


\begin{tabular}{|c|c|}
\hline \multicolumn{2}{|l|}{ 06-CP215 } \\
\hline Type & $\mathrm{SH}$ \\
\hline $\mathrm{n}=$ & 25 \\
\hline $\mathrm{BC}(\mathrm{BNadj})=$ & -0.0062 \\
\hline $\mathrm{BC}(\mathrm{DC})=$ & 0.0013 \\
\hline BC(MS_new) = & 0.0001 \\
\hline delta $=$ & 0.0085 \\
\hline mean of $\ln (x+$ delta $)=$ & -3.577 \\
\hline $\ln \mathrm{sd}=$ & 1.044 \\
\hline UTL K = & 2.442 \\
\hline UTL = & 0.349 \\
\hline Alpha = & 0.025 \\
\hline ProbPlot $\mathrm{R}=$ & 0.922 \\
\hline
\end{tabular}

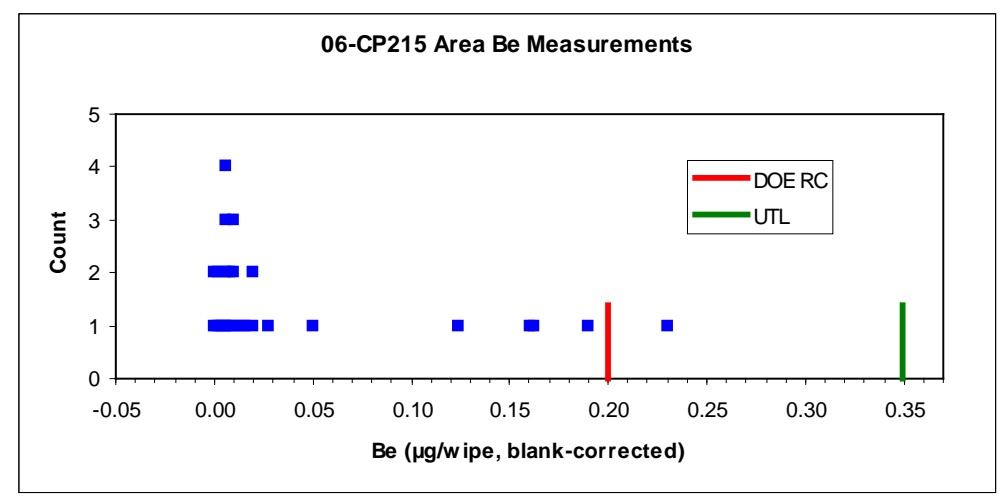

Several of the MS_new measurements in the Area portion of the facility are elevated, with one exceeding the DOE RC. The Be concentration and metal ratios from the bulk sample obtained within the facility are consistent with NTS exterior soils, as are the metal ratios from the wipe sample with the highest Be concentration.

\section{6-CP45}

\begin{tabular}{|c|c|}
\hline \multicolumn{2}{|l|}{ 06-СР45 } \\
\hline IWAs & \\
\hline Type & OF \\
\hline $\mathrm{N}=$ & 67 \\
\hline $\mathrm{n}=$ & 21 \\
\hline $\mathrm{BC}(\mathrm{DC})=$ & 0.0003 \\
\hline delta $=$ & 0.0050 \\
\hline mean of $\ln (x+$ delta $)=$ & -4.856 \\
\hline $\ln \mathrm{sd}=$ & 0.575 \\
\hline $\mathrm{PL} \mathrm{K}=$ & 3.519 \\
\hline PLall = & 0.054 \\
\hline UTL K = & 2.371 \\
\hline UTL = & 0.025 \\
\hline Alpha $=$ & 0.05 \\
\hline ProbPlot $\mathrm{R}=$ & 0.945 \\
\hline
\end{tabular}

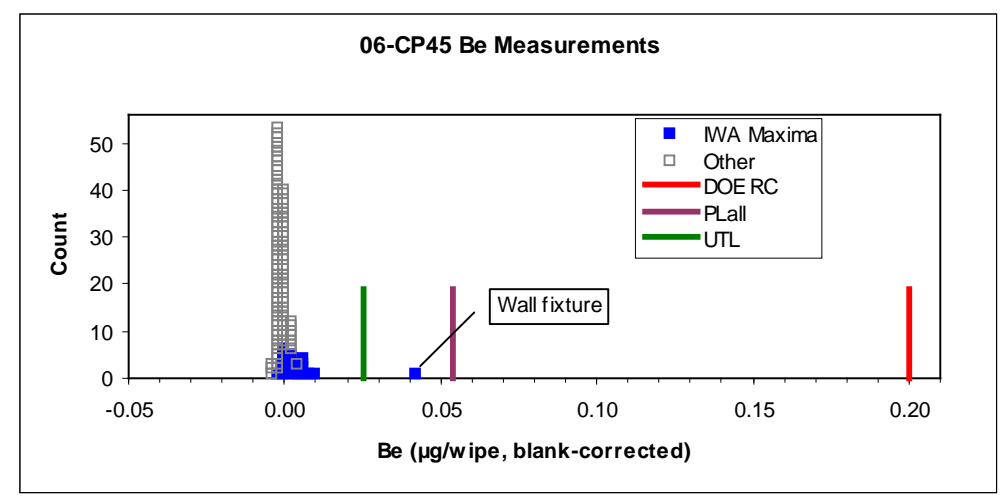

Occasionally there is an anomaly, or "outlier," in the data that begs being pointed out. In this case the high value was obtained on a wall fixture; one might argue whether this is actually part of the normally "touchable" work environment. For this facility the outlier was small enough and there were enough other IWAs that the facility passed anyway. 


\section{6-CP50}

\begin{tabular}{|c|c|}
\hline \multirow{2}{*}{\multicolumn{2}{|c|}{ 06-СР50 }} \\
\hline & \\
\hline Type & $\mathrm{SH}$ \\
\hline $\mathrm{N}=$ & 15 \\
\hline $\mathrm{n}=$ & 6 \\
\hline $\mathrm{BC}(\mathrm{DC})=$ & 0.0003 \\
\hline delta $=$ & 0.0030 \\
\hline mean of $\ln (\mathrm{x}+$ delta $)=$ & -5.261 \\
\hline $\ln s d=$ & 0.394 \\
\hline PL K = & 4.133 \\
\hline PLall $=$ & 0.023 \\
\hline UTL K = & 3.918 \\
\hline UTL = & 0.021 \\
\hline Alpha $=$ & 0.04 \\
\hline ProbPlot $\mathrm{R}=$ & 0.972 \\
\hline
\end{tabular}

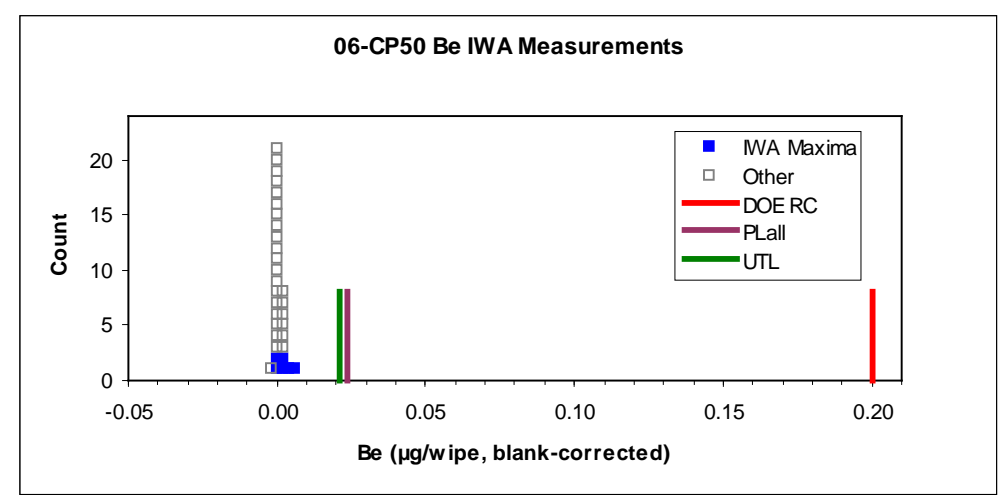

\begin{tabular}{|lcl|}
\hline 06-CP50 & Area & \\
Type & SH \\
$\mathrm{n}=$ & 47 \\
BC(DC) $=$ & 0.0003 \\
delta $=$ & 0.0055 \\
mean of $\ln (\mathrm{x}+$ delta $)=$ & -4.947 \\
$\ln \mathrm{sd}=$ & 0.426 \\
UTL K $=$ & 2.294 \\
UTL $=$ & $\mathbf{0 . 0 1 3}$ \\
Alpha $=$ & 0.01 \\
ProbPlot $\mathrm{R}=$ & 0.891 \\
\hline
\end{tabular}

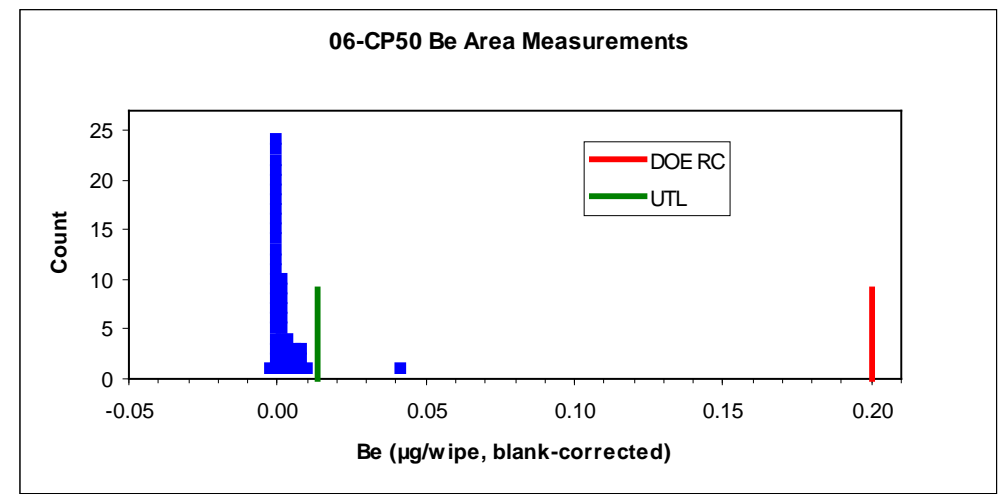

The Area ProbPlot $\mathrm{R}$ value is somewhat lower than one would like to see, due to a relatively high outlier that is nonetheless well below the DOE RC. This should not be considered to be a handicap in this case, since the data values are all very close to zero, and the distribution is likely affected mostly by the rounding of the data in that region. 


\section{6-CP70}

\begin{tabular}{|c|c|}
\hline \multicolumn{2}{|l|}{ 06-СР70 } \\
\hline IWAs & \\
\hline Type & FO \\
\hline $\mathrm{N}=$ & 17 \\
\hline $\mathrm{n}=$ & 9 \\
\hline $\mathrm{BC}(\mathrm{BNadj})=$ & -0.0062 \\
\hline $\mathrm{BC}(\mathrm{DC})=$ & 0.0013 \\
\hline BC(MS_arch) $=$ & 0.0004 \\
\hline delta $=$ & 0.0030 \\
\hline mean of $\ln (x+$ delta $)=$ & -4.516 \\
\hline $\ln \mathrm{sd}=$ & 0.685 \\
\hline $\mathrm{PL} \mathrm{K}=$ & 3.414 \\
\hline PLall $=$ & 0.110 \\
\hline UTL K = & 3.156 \\
\hline UTL = & 0.092 \\
\hline Alpha $=$ & 0.04 \\
\hline ProbPlot $\mathrm{R}=$ & 0.815 \\
\hline
\end{tabular}

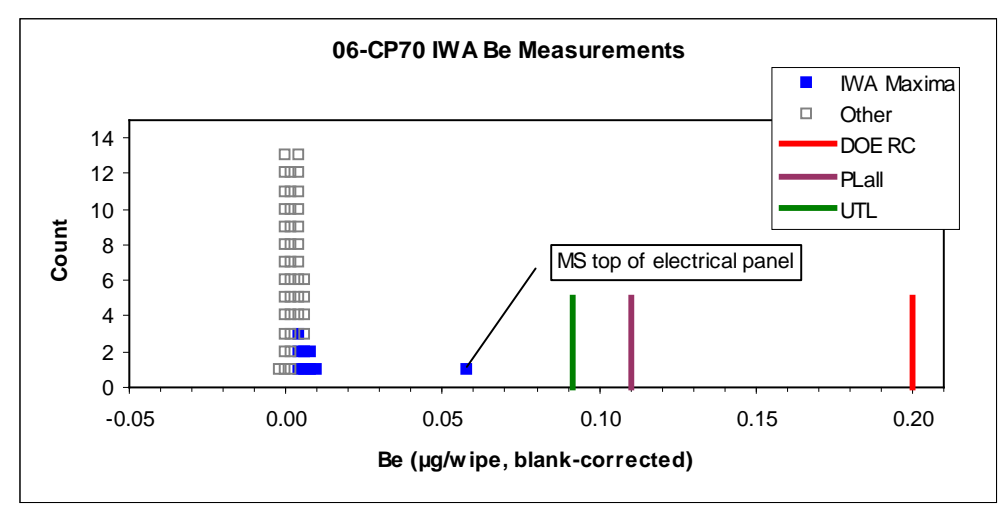

The one relatively high value (MS_arch) was obtained on top of an electrical panel, a location that should perhaps not be considered as part of the normal "touchable" work environment. It was not omitted in this case since PLall is less that the DOE RC even with it included.

\begin{tabular}{|c|c|}
\hline \multicolumn{2}{|l|}{ 06-CP70 } \\
\hline Area & \\
\hline Type & FO \\
\hline $\mathrm{n}=$ & 16 \\
\hline $\mathrm{BC}(\mathrm{BNadj})=$ & -0.0062 \\
\hline $\mathrm{BC}(\mathrm{DC})=$ & 0.0013 \\
\hline BC(MS_new) = & 0.0001 \\
\hline delta $=$ & 0.0085 \\
\hline mean of $\ln (x+$ delta $)=$ & -3.514 \\
\hline $\ln \mathrm{sd}=$ & 1.224 \\
\hline UTL K = & 3.028 \\
\hline UTL $=$ & 1.201 \\
\hline Alpha $=$ & 0.01 \\
\hline ProbPlot $\mathrm{R}=$ & 0.904 \\
\hline
\end{tabular}

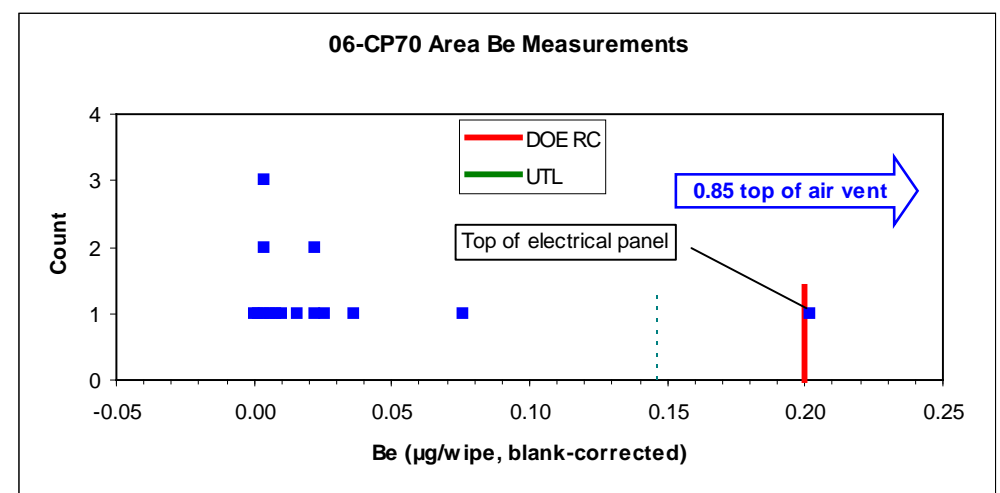

In the equipment bays there were two high values in locations not normally "touchable," one considerably higher than the DOE RC and one just above it, both MS_new. With these values included, the UTL is quite high; with those values omitted, UTL $=0.147$. The Be concentration in the bulk sample obtained in the facility is low, and the metal ratios generally indicate relatively low Be. The metal ratios in the wipe samples with high Be concentrations are consistent with NTS exterior soils. 


\section{6-CP72}

$\square$

06-CP72

$\begin{array}{rc}\text { IWAs } & \\ \text { Type } & \text { FO } \\ \mathrm{N}= & 29 \\ \mathrm{n}= & 8 \\ \mathrm{BC}(\mathrm{DC})= & 0.0003 \\ \text { delta }= & 0.0030 \\ \text { mean of } \ln (\mathrm{x}+\text { delta })= & -4.498 \\ \ln \mathrm{sd}= & 0.597 \\ \mathrm{PL} \mathrm{K}= & 3.978 \\ \text { PLall }= & \mathbf{0 . 1 1 7} \\ \mathrm{UTL} \mathrm{K}= & 3.187 \\ \text { UTL }= & \mathbf{0 . 0 7 2} \\ \text { Alpha }= & 0.05 \\ \text { ProbPlot } \mathrm{R}= & 0.952\end{array}$

\section{6-CP9}

\begin{tabular}{|c|c|}
\hline \multicolumn{2}{|l|}{ 06-CP9 } \\
\hline IWAs & \\
\hline Type & EX \\
\hline $\mathrm{N}=$ & 58 \\
\hline $\mathrm{n}=$ & 18 \\
\hline $\mathrm{BC}(\mathrm{BNadj})=$ & -0.0062 \\
\hline $\mathrm{BC}(\mathrm{DC})=$ & 0.0013 \\
\hline BC(MS_arch $)=$ & 0.0004 \\
\hline delta $=$ & 0.0040 \\
\hline mean of $\ln (x+$ delta $)=$ & -4.716 \\
\hline $\ln \mathrm{sd}=$ & 0.934 \\
\hline PL K = & 3.543 \\
\hline PLall = & 0.241 \\
\hline UTL K = & 2.453 \\
\hline UTL = & 0.085 \\
\hline Alpha $=$ & 0.05 \\
\hline ProbPlot $\mathrm{R}=$ & 0.971 \\
\hline
\end{tabular}
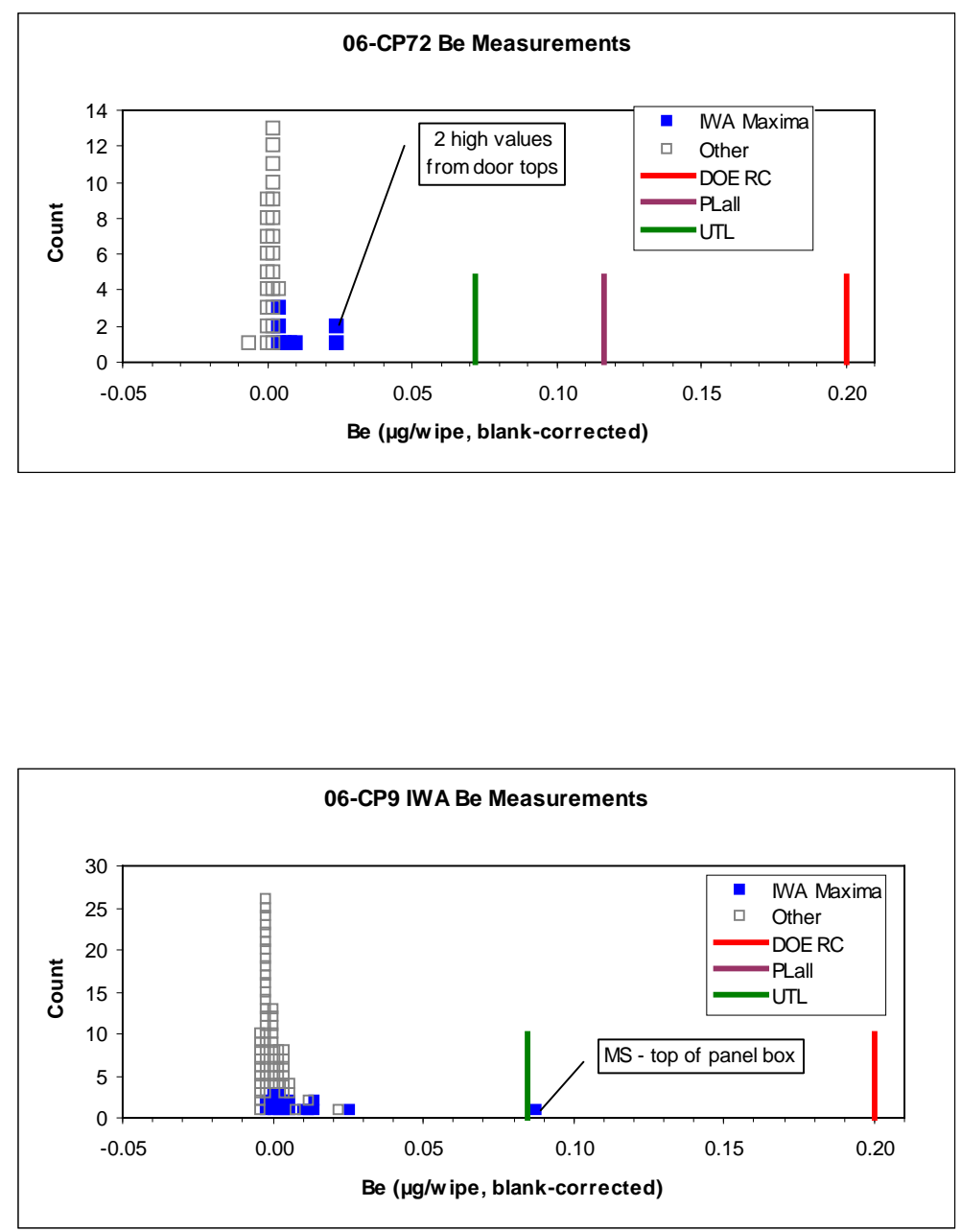


\section{6-СР95А}

\section{$\square$}

06-CP95A

$\begin{array}{rcc}\text { IWAs } & \\ \text { Type } & \text { LA } \\ \mathrm{N}= & 11 \\ \mathrm{n}= & 6 \\ \text { BC(BNadj })= & -0.0062 \\ \text { BC }(\text { DC })= & 0.0013 \\ \text { BC(MS_arch })= & 0.0004 \\ \text { delta }= & 0.0040 \\ \text { Mean of } \ln (\mathrm{x}+\text { delta })= & -4.737 \\ \text { ln sd }= & 0.162 \\ \text { PL K }= & 3.659 \\ \text { PLall }= & \mathbf{0 . 0 1 2} \\ \text { UTL K }= & 3.918 \\ \text { UTL }= & \mathbf{0 . 0 1 3} \\ \text { Alpha }= & 0.04 \\ \text { ProbPlot } \mathrm{R}= & 0.985\end{array}$

\begin{tabular}{|c|c|}
\hline \multicolumn{2}{|l|}{ 06-СР95А } \\
\hline Area & \\
\hline Type & LA \\
\hline $\mathrm{n}=$ & 24 \\
\hline $\mathrm{BC}(\mathrm{BNadj})=$ & -0.0062 \\
\hline $\mathrm{BC}(\mathrm{DC})=$ & 0.0013 \\
\hline BC(MS_arch $)=$ & 0.0004 \\
\hline delta $=$ & 0.0070 \\
\hline mean of $\ln (x+$ delta $)=$ & -5.108 \\
\hline $\ln \mathrm{sd}=$ & 0.728 \\
\hline UTL K = & 2.662 \\
\hline UTL = & 0.035 \\
\hline Alpha $=$ & 0.01 \\
\hline ProbPlot $\mathrm{R}=$ & 0.981 \\
\hline
\end{tabular}
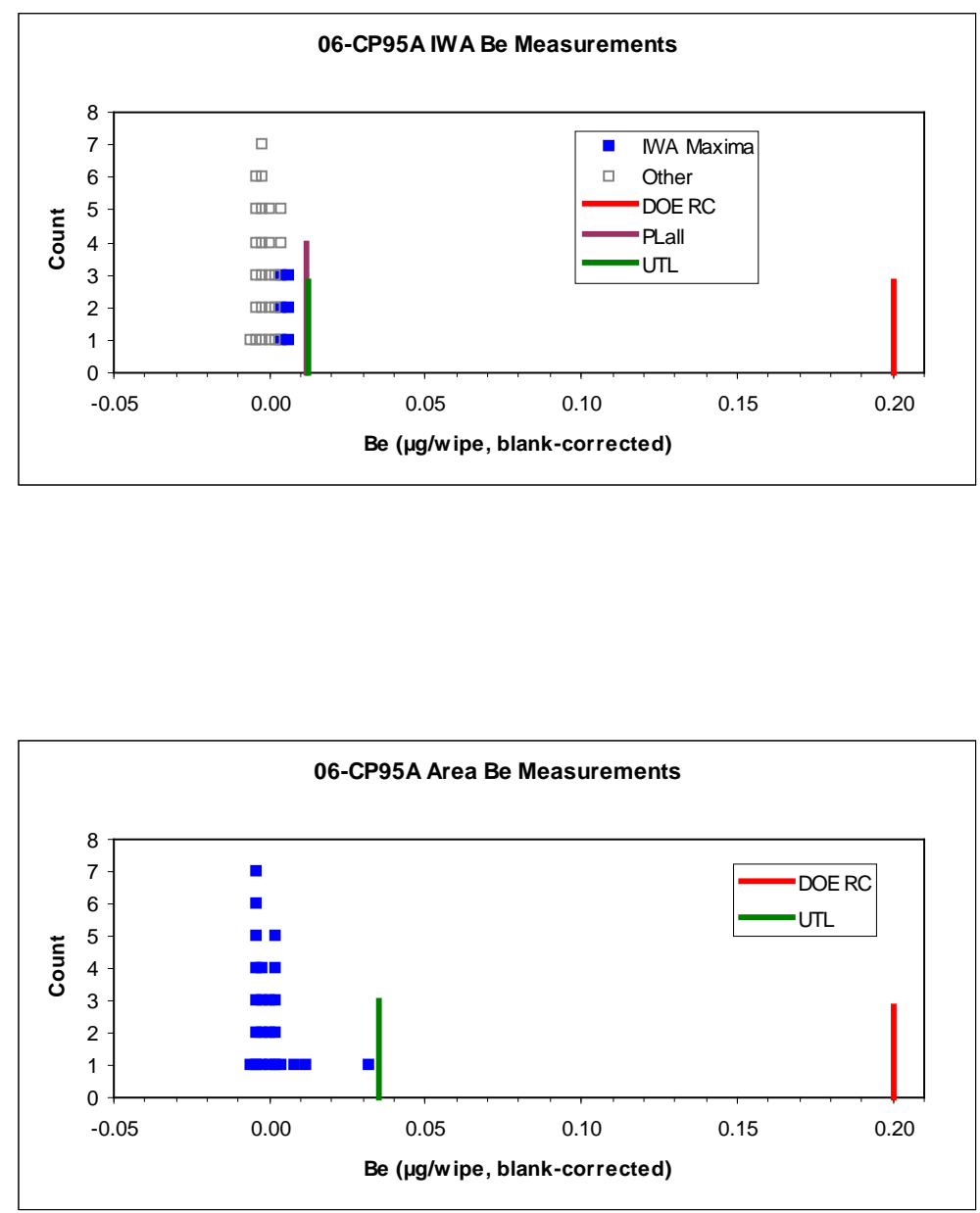


\section{2-45}

\begin{tabular}{|c|c|c|}
\hline \multirow{2}{*}{\multicolumn{3}{|c|}{$12-45$}} \\
\hline & & \\
\hline & Type & QU \\
\hline & $\mathrm{N}=$ & 28 \\
\hline & $\mathrm{n}=$ & 7 \\
\hline & $\mathrm{BC}(\mathrm{BNadj})=$ & -0.0062 \\
\hline & $\mathrm{BC}(\mathrm{DC})=$ & 0.0013 \\
\hline & BC(MS_arch) = & 0.0004 \\
\hline & delta $=$ & 0.0040 \\
\hline & mean of $\ln (x+$ delta $)=$ & -4.030 \\
\hline & $\ln \mathrm{sd}=$ & 1.212 \\
\hline & PL K = & 4.201 \\
\hline & PLall $=$ & 2.882 \\
\hline & UTL K = & 3.400 \\
\hline & UTL = & 1.089 \\
\hline & Alpha = & 0.05 \\
\hline & ProbPlot $\mathrm{R}=$ & 0.930 \\
\hline \multicolumn{3}{|c|}{ omitting door top } \\
\hline & mean of $\ln (x+$ delta $)=$ & -4.285 \\
\hline & $\ln \mathrm{sd}=$ & 0.732 \\
\hline & PL K = & 4.201 \\
\hline & PLall = & 0.295 \\
\hline & UTL K = & 3.400 \\
\hline & UTL $=$ & 0.162 \\
\hline & ProbPlot $\mathrm{R}=$ & 0.975 \\
\hline
\end{tabular}

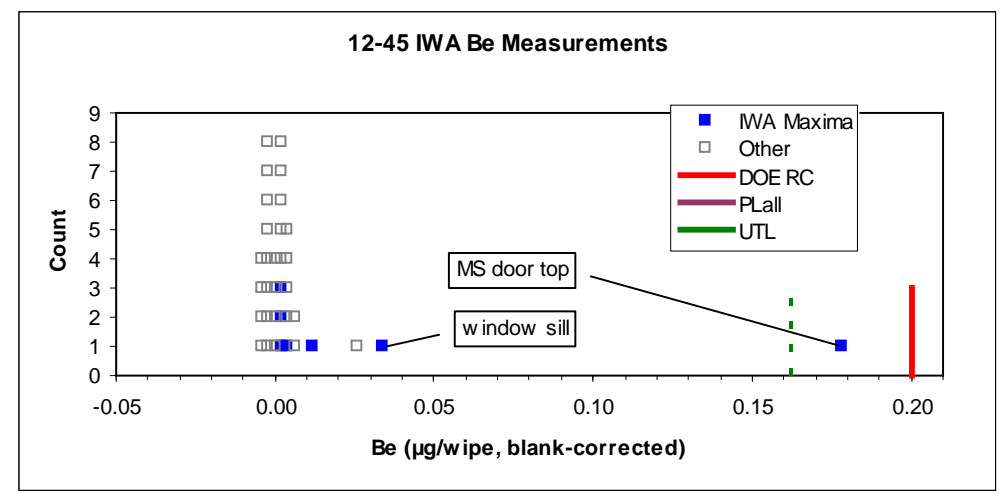

One MS_arch value obtained on a door top is slightly less than the DOE RC; omitting that value, UTL $=0.162$. In addition, the Be concentration and metal ratios in the bulk sample obtained inside the facility are consistent with those in NTS soils. 


\section{2-868}

\begin{tabular}{|c|c|c|}
\hline \multicolumn{3}{|l|}{$12-868$} \\
\hline \multicolumn{3}{|c|}{ IWAs } \\
\hline & Type & SH \\
\hline & $\mathrm{N}=$ & 9 \\
\hline & $\mathrm{n}=$ & 5 \\
\hline & $\mathrm{BC}(\mathrm{BNadj})=$ & -0.0062 \\
\hline & $\mathrm{BC}(\mathrm{DC})=$ & 0.0013 \\
\hline & BC(MS_arch) = & 0.0004 \\
\hline & delta $=$ & 0.0030 \\
\hline & mean of $\ln (x+$ delta $)=$ & -3.361 \\
\hline & $\ln \mathrm{sd}=$ & 0.578 \\
\hline & PL K = & 3.855 \\
\hline & PLall $=$ & 0.319 \\
\hline & UTL K = & 4.485 \\
\hline & UTL $=$ & 0.461 \\
\hline & Alpha $=$ & 0.04 \\
\hline & ProbPlot $\mathrm{R}=$ & 0.972 \\
\hline \multicolumn{3}{|c|}{ UTL cluster sampling analysis } \\
\hline & $\mathrm{F}=$ & 0.61 \\
\hline & delta $=$ & 0.0090 \\
\hline & mean of $\ln (x+$ delta $)=$ & -4.208 \\
\hline & $\ln \mathrm{sd}=$ & 0.854 \\
\hline & UTL K = & 2.465 \\
\hline & UTL $=$ & 0.113 \\
\hline & Alpha $=$ & 0.025 \\
\hline & ProbPlot $\mathrm{R}=$ & 0.957 \\
\hline
\end{tabular}

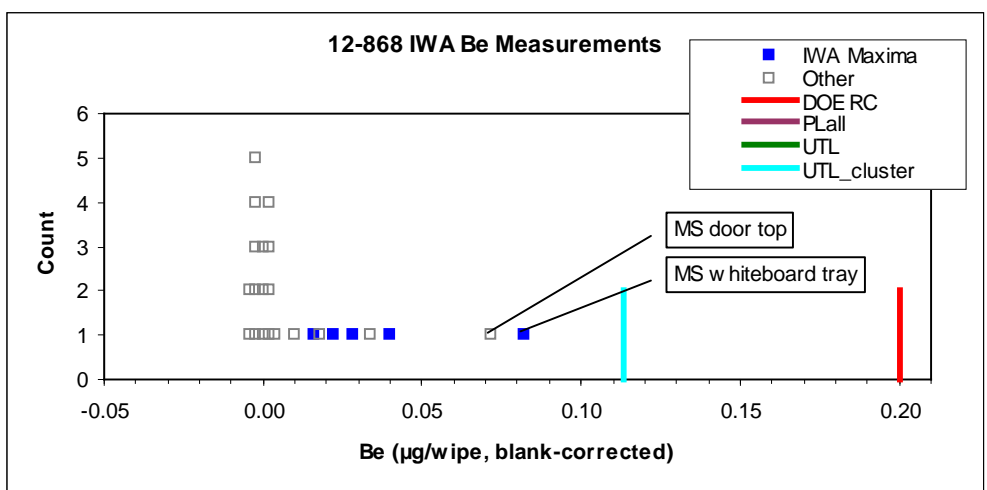

\begin{tabular}{|rc|}
\hline 12-868 Area & \\
Type & SH \\
$\mathrm{n}=$ & 27 \\
BC(BNadj) $=$ & -0.0062 \\
BC(DC) $=$ & 0.0013 \\
BC(MS_arch $)=$ & 0.0004 \\
BC(MS_new $)=$ & 0.0001 \\
delta $=$ & 0.0085 \\
mean of $\ln (\mathrm{x}+$ delta $)=$ & -3.031 \\
$\ln$ sd $=$ & 0.697 \\
UTL K $=$ & 2.402 \\
UTL $=$ & $\mathbf{0 . 2 4 9}$ \\
Alpha $=$ & 0.025 \\
ProbPlot R $=$ & 0.981
\end{tabular}

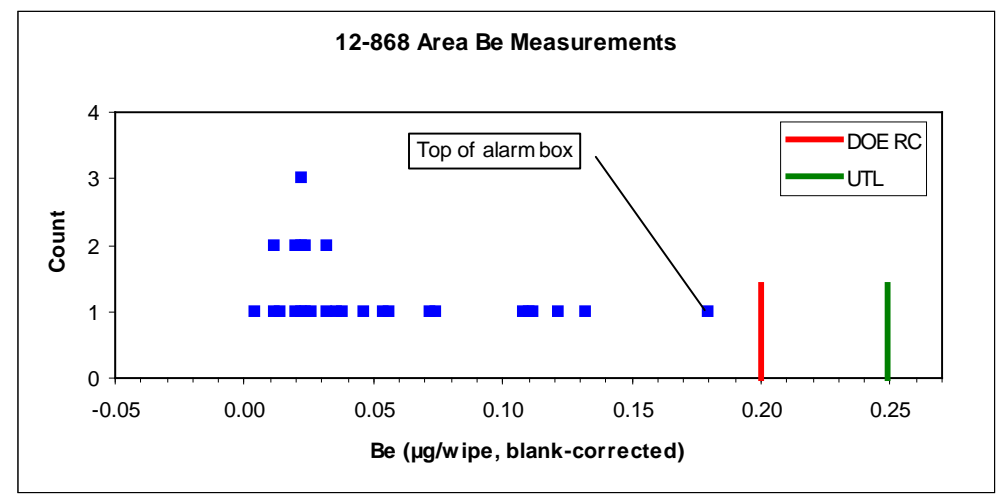

The Area data are rather spread out, so that even if the highest value (at a normally "untouchable location”) is omitted, UTL remains slightly above the DOE RC. Metal ratios in the bulk sample obtained inside the building are consistent with those in NTS soils. 


\section{3-1000}

\begin{tabular}{|c|c|}
\hline \multicolumn{2}{|l|}{$23-1000$} \\
\hline IWAs* & \\
\hline Type & FO \\
\hline $\mathrm{N}=$ & 54 \\
\hline $\mathrm{n}=$ & 14 \\
\hline $\mathrm{BC}(\mathrm{DC})=$ & 0.0003 \\
\hline delta $=$ & 0.0030 \\
\hline mean of $\ln (x+$ delta $)=$ & -5.853 \\
\hline $\ln \mathrm{sd}=$ & 0.495 \\
\hline $\mathrm{PL} \mathrm{K}=$ & 3.712 \\
\hline PLall $=$ & 0.015 \\
\hline UTL K = & 2.614 \\
\hline $\mathbf{U T L}=$ & 0.007 \\
\hline Alpha $=$ & 0.05 \\
\hline ProbPlot $\mathrm{R}=$ & 0.959 \\
\hline
\end{tabular}

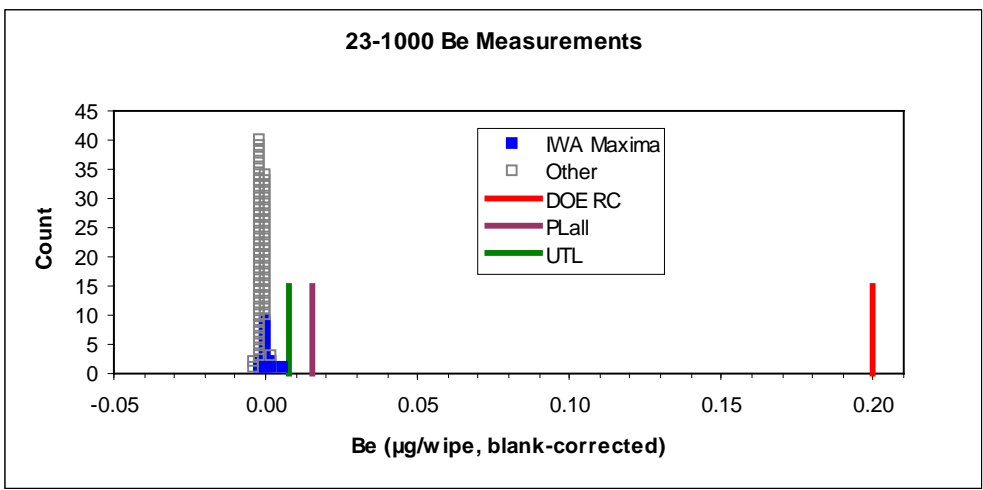

In some cases there is a small Area-like portion of a facility, such as a storage area, conference room, or classroom, which for convenience is simply divided into IWA-size portions and included with the IWAs.

\section{3-1001}

\begin{tabular}{|c|c|}
\hline \multicolumn{2}{|l|}{ 23-1001 } \\
\hline IWAs & \\
\hline Type & FO \\
\hline $\mathrm{N}=$ & 32 \\
\hline $\mathrm{n}=$ & 11 \\
\hline $\mathrm{BC}(\mathrm{DC})=$ & 0.0003 \\
\hline delta $=$ & 0.003 \\
\hline mean of $\ln (x+$ delta $)=$ & -5.246 \\
\hline $\ln \mathrm{sd}=$ & 0.772 \\
\hline $\mathrm{PL} \mathrm{K}=$ & 3.602 \\
\hline PLall = & 0.082 \\
\hline UTL K = & 2.815 \\
\hline UTL $=$ & 0.043 \\
\hline Alpha $=$ & 0.05 \\
\hline ProbPlot $\mathrm{R}=$ & 0.963 \\
\hline
\end{tabular}

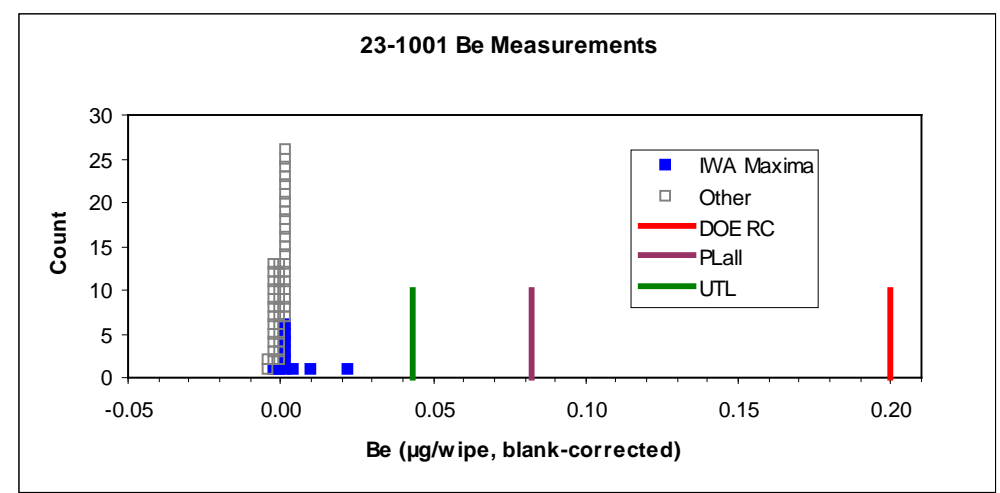




\section{3-1002}

\begin{tabular}{|c|c|}
\hline \multicolumn{2}{|l|}{ 23-1002 } \\
\hline Area & \\
\hline Type & FO \\
\hline $\mathrm{n}=$ & 10 \\
\hline $\mathrm{BC}(\mathrm{BNadj})=$ & -0.0062 \\
\hline delta $=$ & 0.0080 \\
\hline mean of $\ln (x+$ delta $)=$ & -4.806 \\
\hline $\ln \mathrm{sd}=$ & 0.414 \\
\hline UTL K = & 3.031 \\
\hline UTL = & 0.021 \\
\hline Alpha $=$ & 0.05 \\
\hline ProbPlot $\mathrm{R}=$ & 0.974 \\
\hline
\end{tabular}

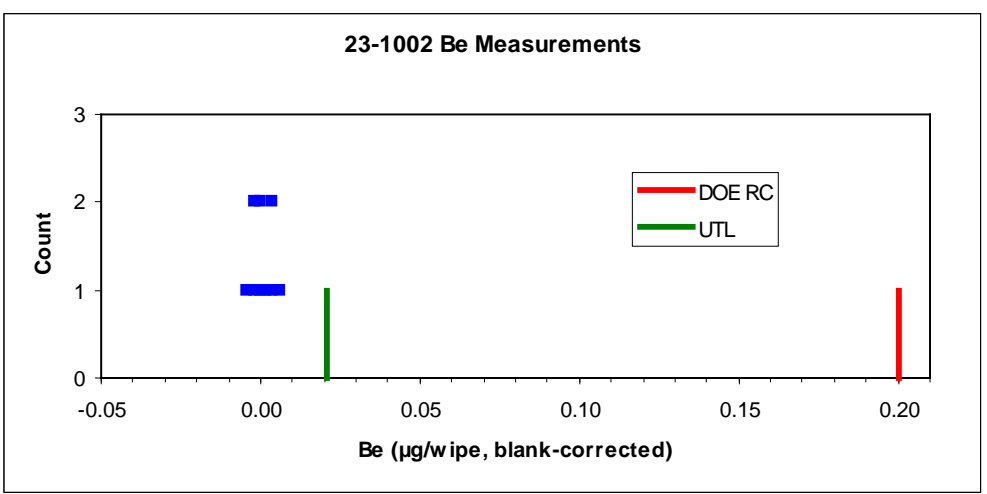

\section{3-1010}

\begin{tabular}{|c|c|}
\hline \multicolumn{2}{|l|}{ 23-1010 } \\
\hline IWAs & \\
\hline Type & $\mathrm{SH}$ \\
\hline $\mathrm{N}=$ & 16 \\
\hline $\mathrm{n}=$ & 8 \\
\hline BC $($ BNadj $)=$ & -0.0062 \\
\hline BC(MS_arch $)=$ & 0.0004 \\
\hline delta $=$ & 0.0030 \\
\hline mean of $\ln (x+$ delta $)=$ & -4.070 \\
\hline $\ln \mathrm{sd}=$ & 1.487 \\
\hline $\mathrm{PL} \mathrm{K}=$ & 3.374 \\
\hline PLall $=$ & 2.578 \\
\hline UTL K = & 3.187 \\
\hline UTL $=$ & 1.953 \\
\hline Alpha $=$ & 0.05 \\
\hline ProbPlot $\mathrm{R}=$ & 0.899 \\
\hline \multicolumn{2}{|c|}{ UTL cluster sampling analysis } \\
\hline $\mathrm{F}=$ & 2.79 \\
\hline delta $=$ & 0.0090 \\
\hline mean of $\ln (x+$ delta $)=$ & -4.542 \\
\hline $\operatorname{tau}^{\wedge} 2=$ & 0.259 \\
\hline $\operatorname{sig} \wedge 2=$ & 0.578 \\
\hline $\ln s d=$ & 0.915 \\
\hline UTL K = & 2.506 \\
\hline UTL $=$ & 0.096 \\
\hline Alpha $=$ & 0.05 \\
\hline ProbPlot $\mathrm{R}=$ & 0.912 \\
\hline
\end{tabular}

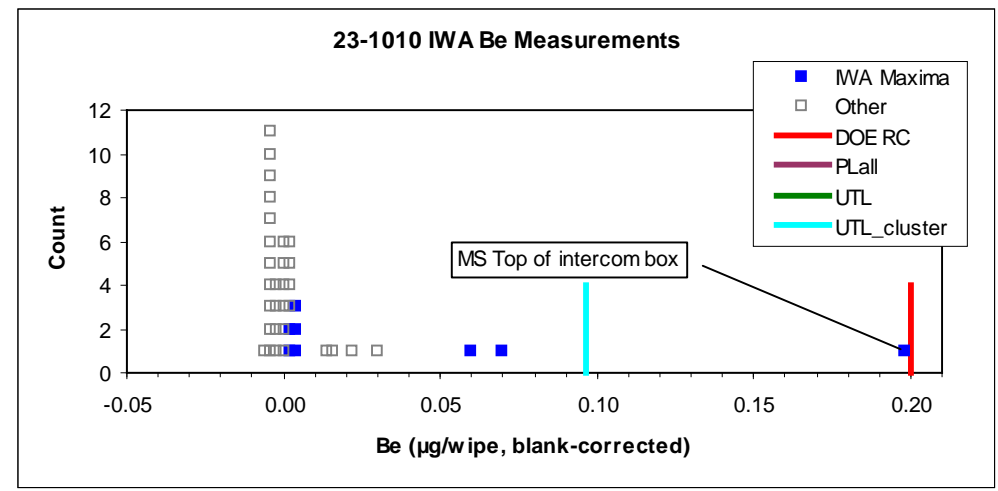


23-109

\begin{tabular}{|c|c|}
\hline \multicolumn{2}{|l|}{ 23-109 } \\
\hline IWAs & \\
\hline Type & ST \\
\hline $\mathrm{N}=$ & 32 \\
\hline $\mathrm{n}=$ & 9 \\
\hline $\mathrm{BC}(\mathrm{DC})=$ & 0.0003 \\
\hline delta $=$ & 0.0030 \\
\hline mean of $\ln (x+$ delta $)=$ & -5.144 \\
\hline $\ln \mathrm{sd}=$ & 0.486 \\
\hline $\mathrm{PL} \mathrm{K}=$ & 3.870 \\
\hline PLall = & 0.035 \\
\hline UTL K = & 3.031 \\
\hline UTL = & 0.022 \\
\hline Alpha & 0.05 \\
\hline ProbPlot $\mathrm{R}=$ & 0.975 \\
\hline
\end{tabular}

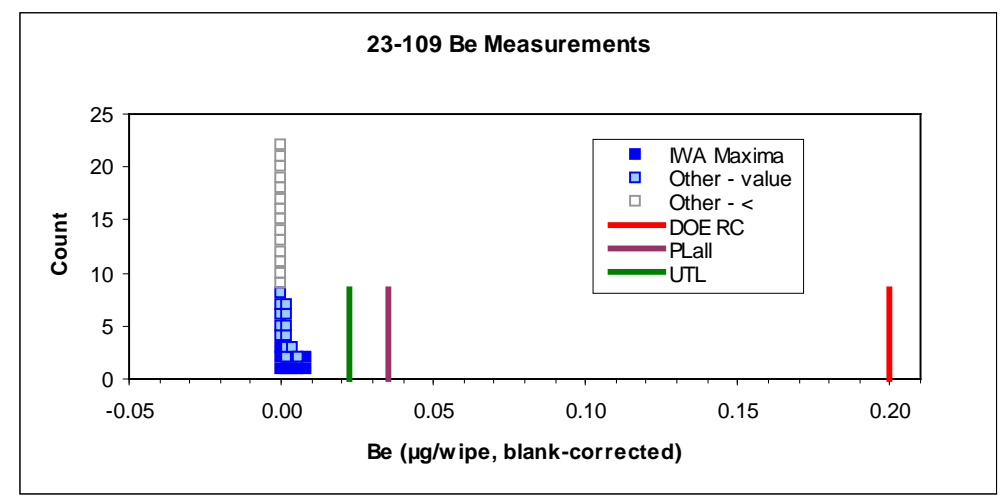

\section{3-1103}

\begin{tabular}{|c|c|}
\hline \multirow{2}{*}{\multicolumn{2}{|c|}{ 23-1103 }} \\
\hline & \\
\hline Tyре & FO \\
\hline $\mathrm{N}=$ & 28 \\
\hline $\mathrm{n}=$ & 5 \\
\hline $\mathrm{BC}(\mathrm{BNadj})=$ & -0.0062 \\
\hline BC(MS_arch $)=$ & 0.0004 \\
\hline delta $=$ & 0.0030 \\
\hline mean of $\ln (x+$ delta $)=$ & -4.930 \\
\hline $\ln \mathrm{sd}=$ & 0.889 \\
\hline PL K = & 3.760 \\
\hline PLall $=$ & 0.201 \\
\hline UTL K = & 3.031 \\
\hline UTL $=$ & 0.104 \\
\hline Alpha $=$ & 0.05 \\
\hline ProbPlot $\mathrm{R}=$ & 0.883 \\
\hline
\end{tabular}

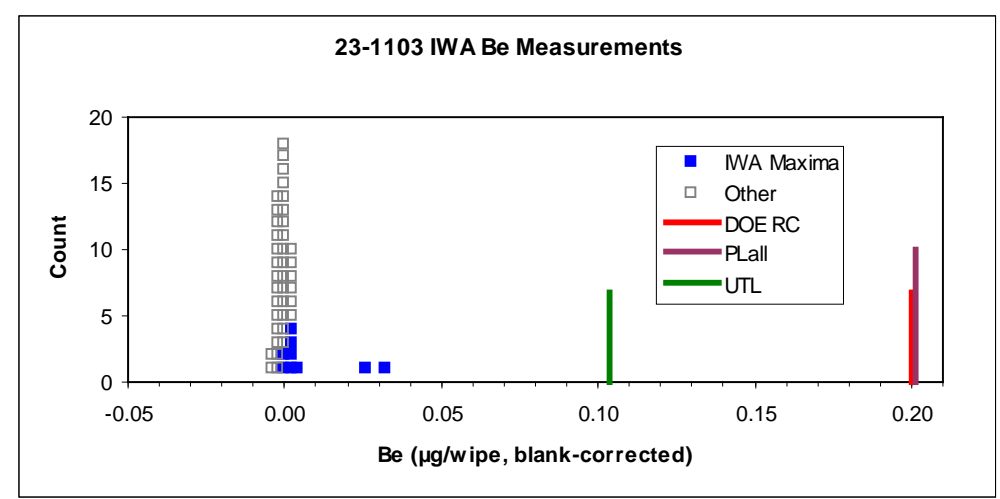

The low ProbPlot $\mathrm{R}$ is due to the two relatively isolated values. 
23-111

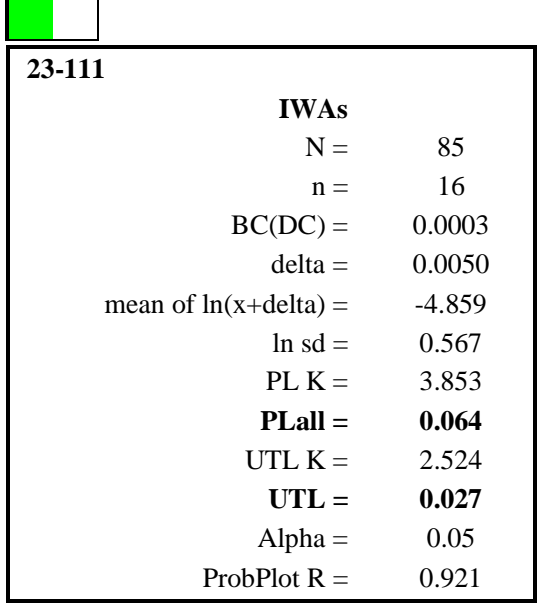

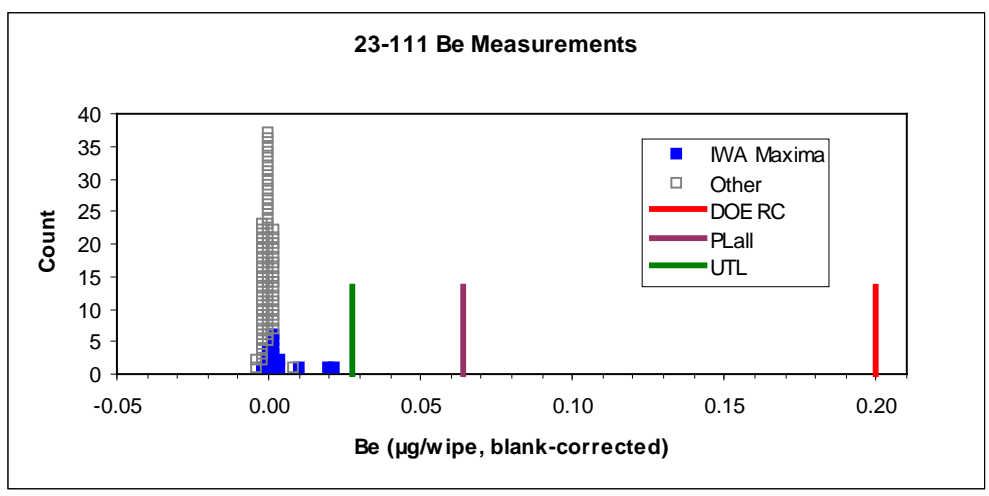

\section{3-113}

\begin{tabular}{|c|c|}
\hline \multicolumn{2}{|l|}{ 23-113 } \\
\hline \multicolumn{2}{|l|}{ IWAs } \\
\hline Type & OF \\
\hline $\mathrm{N}=$ & 23 \\
\hline $\mathrm{n}=$ & 7 \\
\hline $\mathrm{BC}(\mathrm{BNadj})=$ & -0.0062 \\
\hline BC(MS_arch) = & 0.0004 \\
\hline delta $=$ & 0.005 \\
\hline mean of $\ln (x+$ delta $)=$ & -3.453 \\
\hline $\ln \mathrm{sd}=$ & 1.755 \\
\hline PL K = & 3.811 \\
\hline PLall $=$ & 36.665 \\
\hline UTL K = & 3.400 \\
\hline $\mathbf{U T L}=$ & 12.352 \\
\hline Alpha $=$ & 0.05 \\
\hline ProbPlot $\mathrm{R}=$ & 0.957 \\
\hline \multirow{2}{*}{\multicolumn{2}{|c|}{$\begin{array}{l}\text { UTL cluster sampling } \\
\text { omitting video room high value }\end{array}$}} \\
\hline & \\
\hline $\mathrm{F}=$ & 0.97 \\
\hline delta $=$ & 0.0085 \\
\hline mean of $\ln (x+$ delta $)=$ & -4.946 \\
\hline $\ln s d=$ & 1.079 \\
\hline UTL K = & 2.232 \\
\hline $\mathbf{U T L}=$ & 0.071 \\
\hline ProbPlot $\mathrm{R}=$ & 0.945 \\
\hline
\end{tabular}

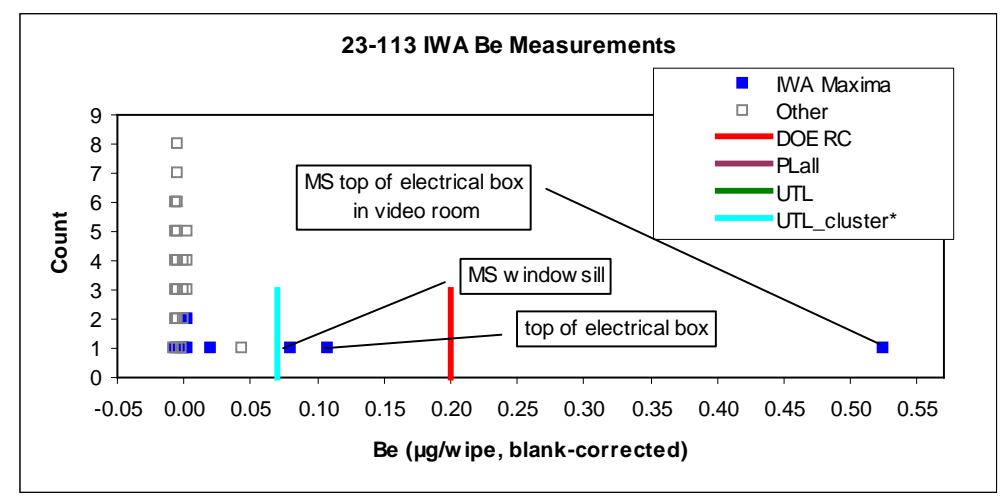

There is a very high value (MS_arch) on the top of an electrical power box in a video room that is rarely entered. Omitting that value, UTL_cluster $=0.071$.

Be concentrations and metal ratios in two bulk samples obtained in that video room show relatively low Be concentrations compared with NTS soils. 


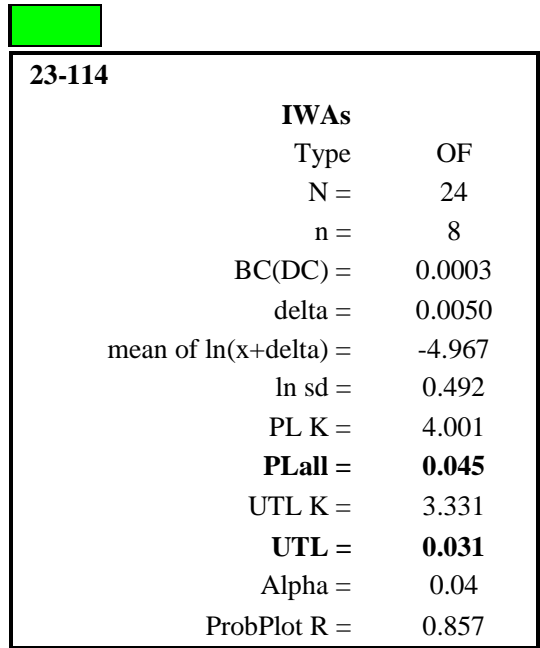

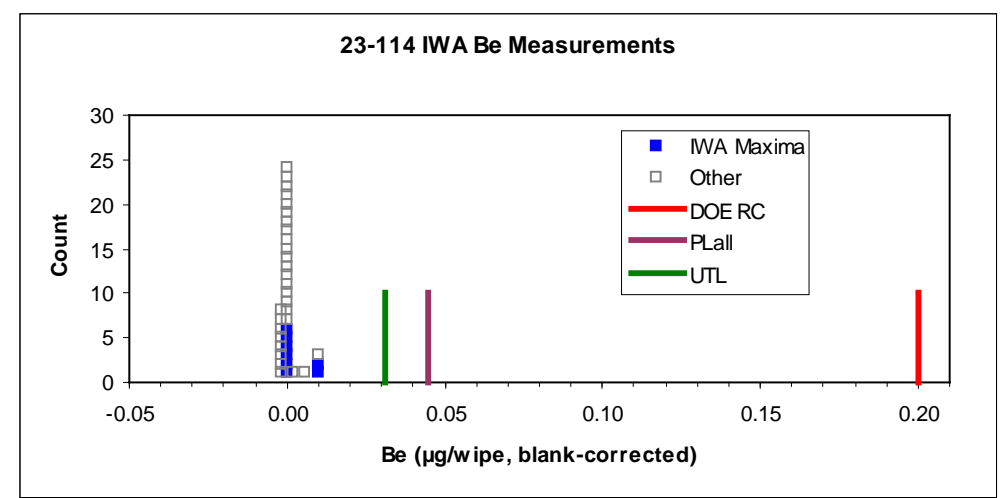

The relatively low ProbPlot R for IWAs reflects the curious data configuration of the IWA maxima. The values are all quite low, though, so there is no concern.

\begin{tabular}{|rc|}
\hline 23-114 & \\
Typea & \\
$\mathrm{n}=$ & OF \\
shift $=$ & 0.0003 \\
delta $=$ & 0.0020 \\
mean of $\ln (\mathrm{x}+$ delta $)=$ & -6.235 \\
UTL $=$ & 0.958 \\
UTL $=$ & 3.028 \\
Alpha $=$ & $\mathbf{0 . 0 3 4}$ \\
ProbPlot $\mathrm{R}=$ & 0.01 \\
& 0.970 \\
\hline
\end{tabular}

\section{3-117}

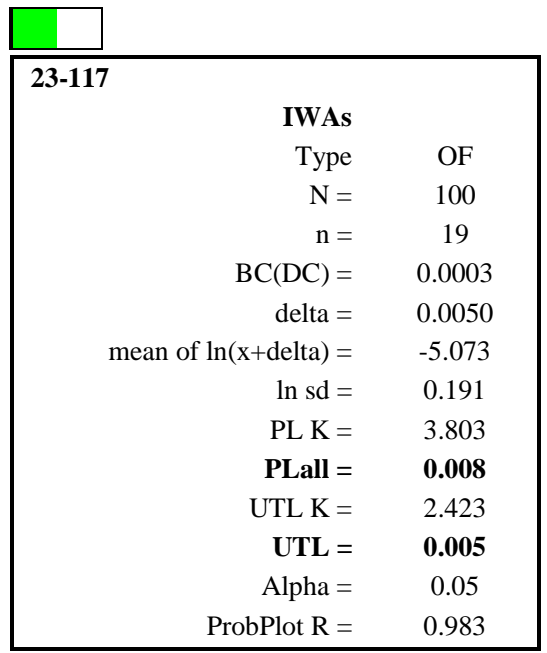
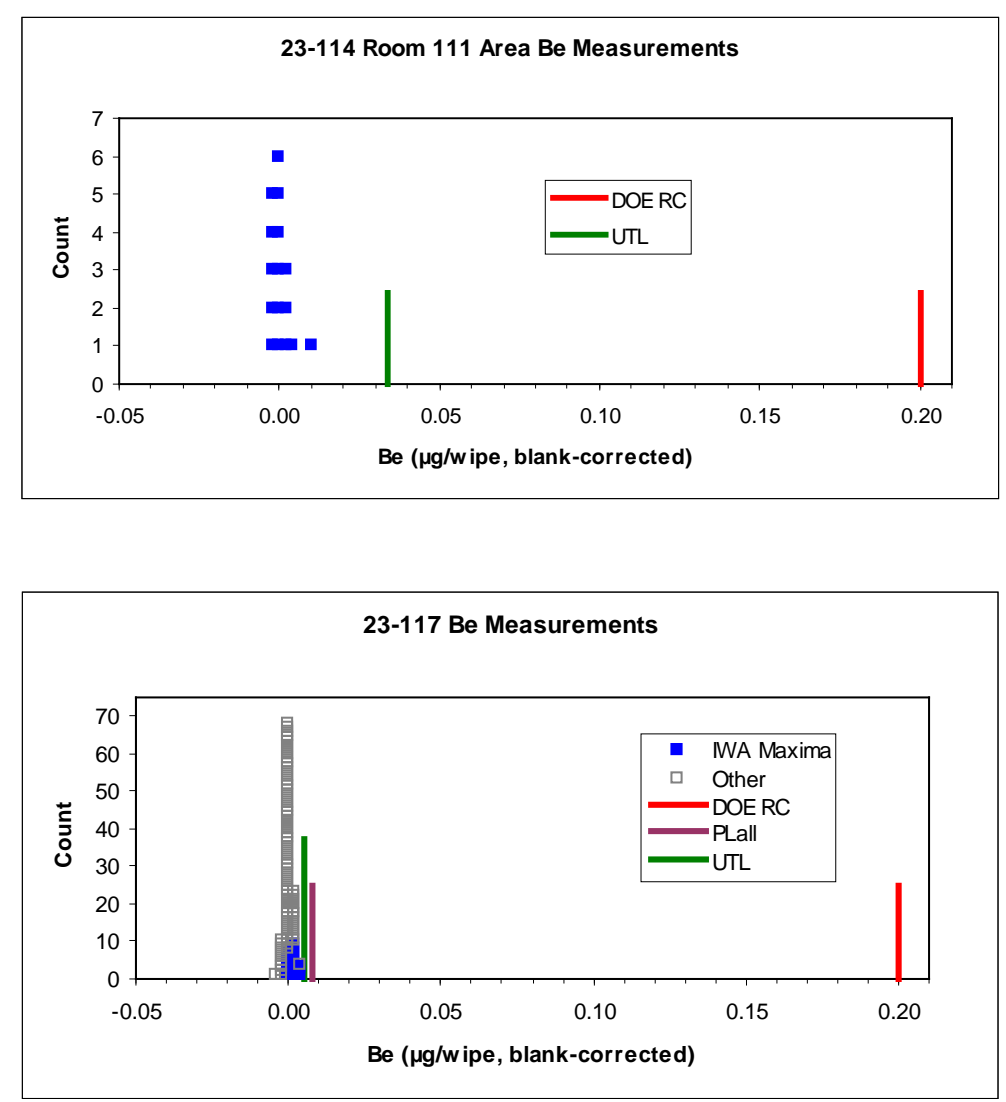
23-118

\begin{tabular}{|c|c|}
\hline \multicolumn{2}{|l|}{$23-118$} \\
\hline IWAs & \\
\hline Type & FO \\
\hline $\mathrm{N}=$ & 26 \\
\hline $\mathrm{n}=$ & 9 \\
\hline BC $($ BNadj $)=$ & -0.0062 \\
\hline BC(MS_arch $)=$ & 0.0004 \\
\hline delta $=$ & 0.0030 \\
\hline mean of $\ln (x+$ delta $)=$ & -4.739 \\
\hline $\ln \mathrm{sd}=$ & 0.370 \\
\hline $\mathrm{PL} \mathrm{K}=$ & 3.884 \\
\hline PLall $=$ & 0.034 \\
\hline UTL K = & 3.331 \\
\hline UTL = & 0.027 \\
\hline Alpha $=$ & 0.04 \\
\hline ProbPlot $\mathrm{R}=$ & 0.949 \\
\hline
\end{tabular}

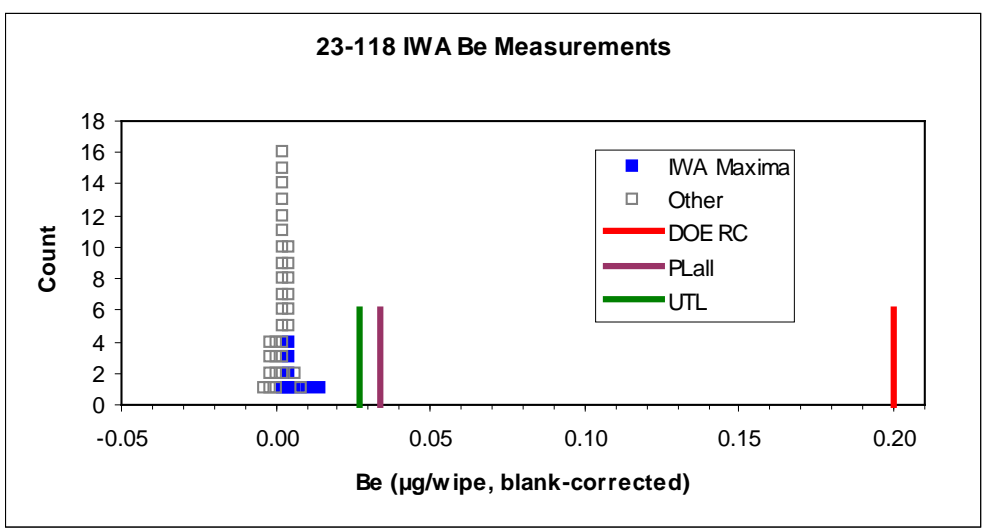

\begin{tabular}{|rc|}
\hline 23-118 & \\
Typea & \\
$\mathrm{n}=$ & 26 \\
BC(BNadj $)=$ & -0.0062 \\
BC(MS_arch $)=$ & 0.0004 \\
delta $=$ & 0.0080 \\
mean of $\ln (\mathrm{x}+$ delta $)=$ & -4.336 \\
UTL $\mathrm{K}=$ & 2.606 \\
UTL $=$ & $\mathbf{0 . 0 5 5}$ \\
Alpha $=$ & 0.01 \\
ProbPlot $\mathrm{R}=$ & 0.981 \\
\hline
\end{tabular}

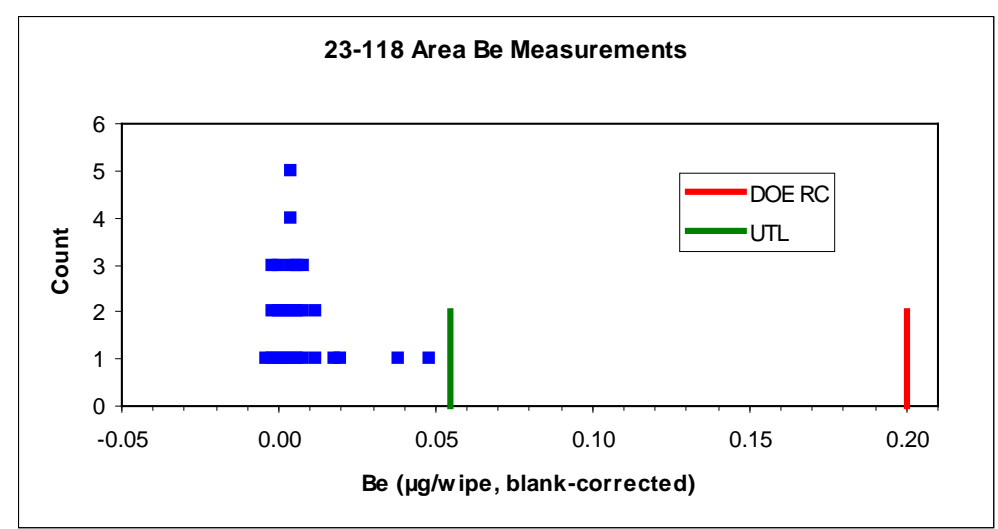




\section{3-128}

\begin{tabular}{|c|c|c|}
\hline \multirow{2}{*}{\multicolumn{3}{|c|}{ 23-128 }} \\
\hline & & \\
\hline & Type & ST \\
\hline & $\mathrm{N}=$ & 13 \\
\hline & $\mathrm{n}=$ & 8 \\
\hline & $\mathrm{BC}(\mathrm{BNadj})=$ & -0.0062 \\
\hline & $\mathrm{BC}(\mathrm{DC})=$ & 0.0013 \\
\hline & BC(MS_arch) $=$ & 0.0004 \\
\hline & delta $=$ & 0.0030 \\
\hline & mean of $\ln (x+$ delta $)=$ & -4.268 \\
\hline & $\ln \mathrm{sd}=$ & 0.871 \\
\hline & PL K = & 3.244 \\
\hline & PLall = & 0.233 \\
\hline & UTL K = & 3.331 \\
\hline & UTL = & 0.252 \\
\hline & Alpha $=$ & 0.04 \\
\hline & ProbPlot $\mathrm{R}=$ & 0.958 \\
\hline \multicolumn{3}{|c|}{ UTL cluster sampling analysis } \\
\hline & $\mathrm{F}=$ & 2.39 \\
\hline & delta $=$ & 0.0090 \\
\hline & mean of $\ln (x+$ delta $)=$ & -4.421 \\
\hline & $\operatorname{tau} \wedge 2=$ & 0.055 \\
\hline & $\operatorname{sig} \wedge 2=$ & 0.199 \\
\hline & $\ln \mathrm{sd}=$ & 0.504 \\
\hline & UTL K = & 2.687 \\
\hline & UTL = & 0.038 \\
\hline & Alpha = & 0.025 \\
\hline & ProbPlot $\mathrm{R}=$ & 0.929 \\
\hline
\end{tabular}

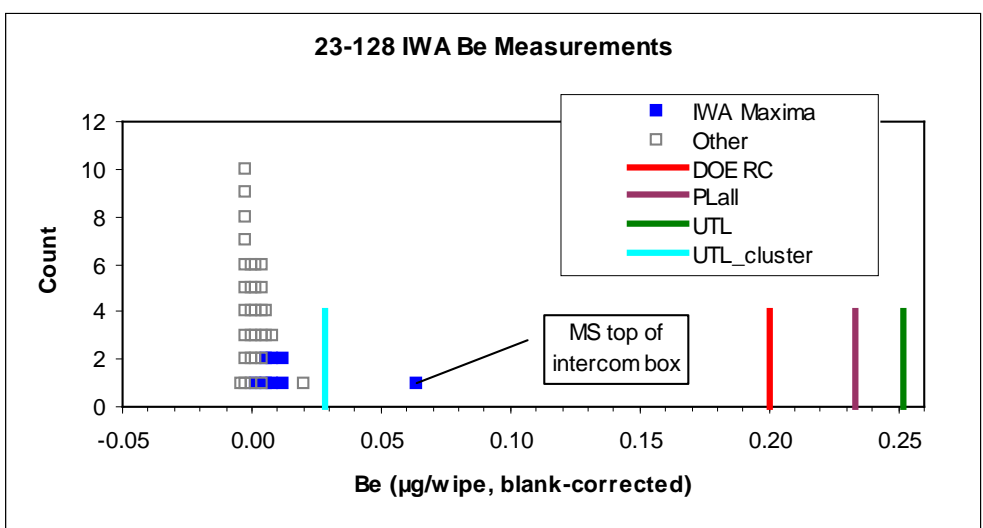

The relatively high value (MS_arch) is in a difficult-to-reach location. This data value would be a candidate for omission; however, UTL_cluster which takes into account all data values, not just the IWA maxima, is less than the DOE RC.

\begin{tabular}{|c|c|}
\hline \multirow{2}{*}{\multicolumn{2}{|c|}{ 23-128 }} \\
\hline & \\
\hline Type & $\mathrm{ST}$ \\
\hline $\mathrm{n}=$ & 40 \\
\hline $\mathrm{BC}(\mathrm{BNadj})=$ & -0.0062 \\
\hline $\mathrm{BC}(\mathrm{DC})=$ & 0.0013 \\
\hline BC(MS_new $)=$ & 0.0001 \\
\hline delta $=$ & 0.0085 \\
\hline mean of $\ln (x+$ delta $)=$ & -4.283 \\
\hline $\ln \mathrm{sd}=$ & 0.700 \\
\hline UTL K = & 2.128 \\
\hline UTL = & 0.053 \\
\hline Alpha $=$ & 0.025 \\
\hline ProbPlot $\mathrm{R}=$ & 0.988 \\
\hline
\end{tabular}

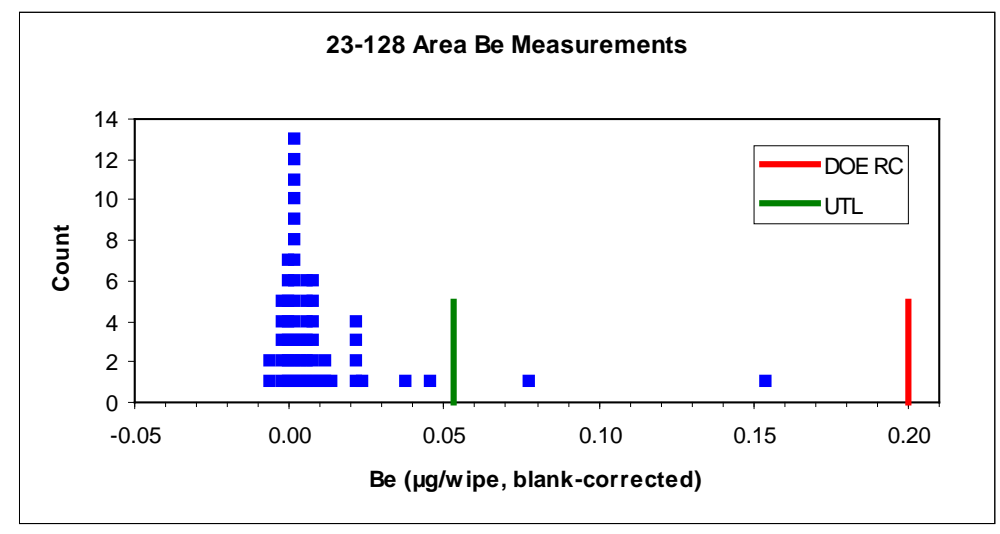

Also, four bulk samples were obtained inside this facility; the Be concentrations and metal ratios were consistent with NTS soils for all of these samples. 
23-132

$\square$

\begin{tabular}{rc|}
\hline 23-132 & \\
IWAs & \\
Type & OF \\
$\mathrm{N}=$ & 38 \\
$\mathrm{n}=$ & 10 \\
BC(DC) $=$ & 0.0003 \\
delta $=$ & 0.0050 \\
mean of $\ln (\mathrm{x}+$ delta $)=$ & -4.782 \\
$\ln \mathrm{sd}=$ & 0.307 \\
$\mathrm{PL} \mathrm{K}=$ & 3.853 \\
PLall $=$ & $\mathbf{0 . 0 2 2}$ \\
UTL K $=$ & 2.911 \\
UTL $=$ & $\mathbf{0 . 0 1 6}$ \\
Alpha $=$ & 0.05 \\
ProbPlot $\mathrm{R}=$ & 0.962 \\
\hline
\end{tabular}

\section{3-133}

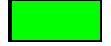

\begin{tabular}{|c|c|}
\hline \multicolumn{2}{|l|}{ 23-133 } \\
\hline IWAs & \\
\hline Type & ST \\
\hline $\mathrm{N}=$ & 4 \\
\hline $\mathrm{n}=$ & 3 \\
\hline $\mathrm{BC}(\mathrm{BNadj})=$ & -0.0062 \\
\hline $\mathrm{BC}(\mathrm{MS})=$ & 0.0004 \\
\hline delta $=$ & 0.003 \\
\hline mean of $\ln (x+$ delta $)=$ & -4.639 \\
\hline $\ln \mathrm{sd}=$ & 0.514 \\
\hline PL K = & 3.833 \\
\hline PLall = & 0.066 \\
\hline UTL K = & 8.591 \\
\hline UTL = & 0.795 \\
\hline Alpha $=$ & 0.04 \\
\hline ProbPlot $\mathrm{R}=$ & 0.987 \\
\hline
\end{tabular}

\begin{tabular}{|c|c|}
\hline \multicolumn{2}{|l|}{ 23-133 } \\
\hline Type & ST \\
\hline $\mathrm{n}=$ & 28 \\
\hline $\mathrm{BC}(\mathrm{BNadj})=$ & -0.0062 \\
\hline $\mathrm{BC}(\mathrm{MS})=$ & 0.0004 \\
\hline delta $=$ & 0.008 \\
\hline mean of $\ln (x+$ delta $)=$ & -4.186 \\
\hline $\ln \mathrm{sd}=$ & 0.750 \\
\hline UTL K = & 2.558 \\
\hline UTL = & 0.095 \\
\hline Alpha $=$ & 0.01 \\
\hline ProbPlot $\mathrm{R}=$ & 0.962 \\
\hline
\end{tabular}
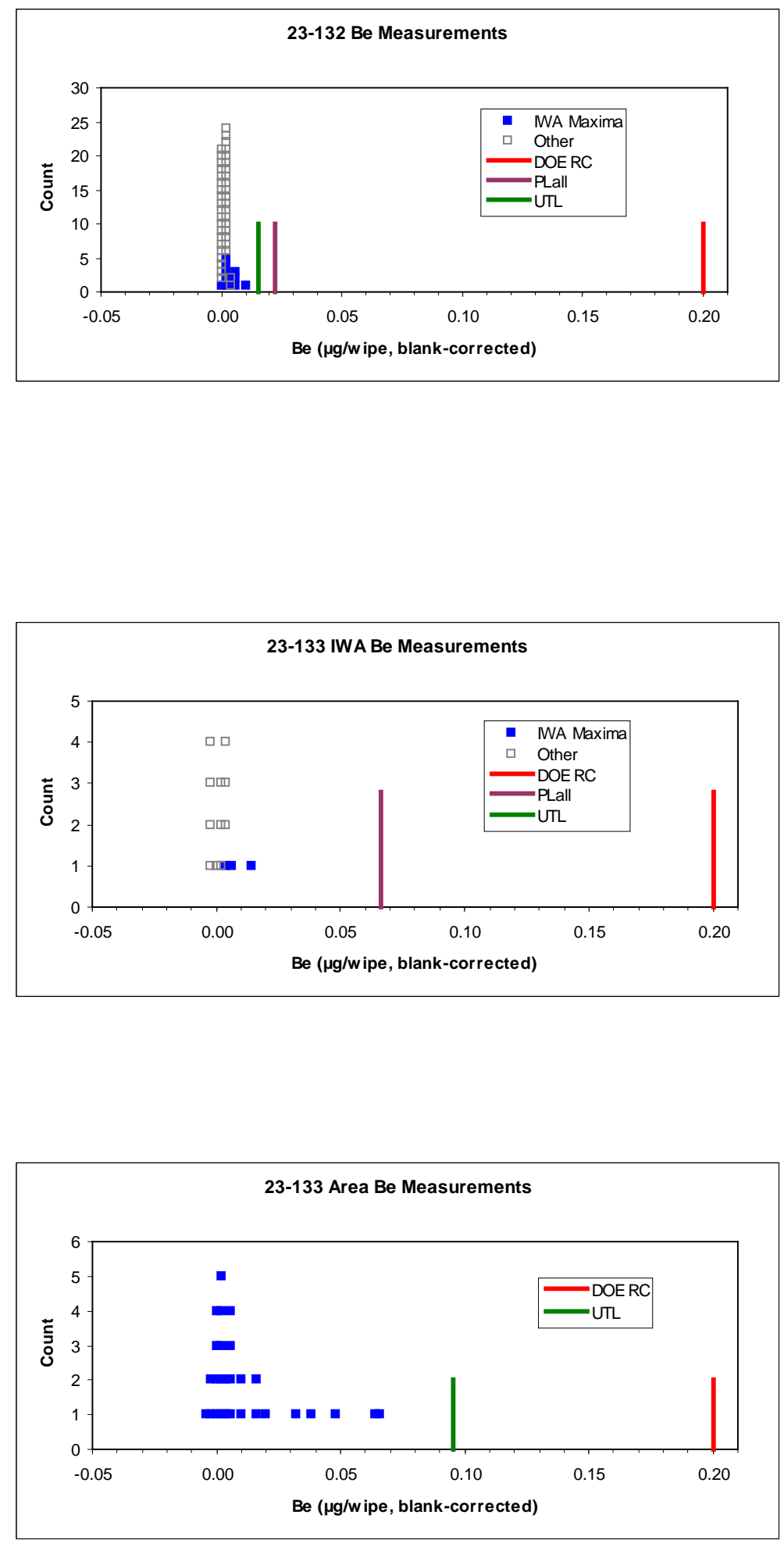
23-143

\begin{tabular}{|c|c|c|}
\hline \multirow{2}{*}{\multicolumn{3}{|c|}{$23-143$}} \\
\hline & & \\
\hline & Type & OF \\
\hline & $\mathrm{N}=$ & 31 \\
\hline & $\mathrm{n}=$ & 9 \\
\hline & $\mathrm{BC}(\mathrm{BNadj})=$ & -0.0062 \\
\hline & delta $=$ & 0.0050 \\
\hline & mean of $\ln (x+$ delta $)=$ & -4.603 \\
\hline & $\ln \mathrm{sd}=$ & 0.759 \\
\hline & $\mathrm{PL} \mathrm{K}=$ & 3.845 \\
\hline & PLall = & 0.180 \\
\hline & UTL K = & 3.031 \\
\hline & UTL $=$ & 0.095 \\
\hline & Alpha = & 0.05 \\
\hline & ProbPlot $\mathrm{R}=$ & 0.886 \\
\hline
\end{tabular}

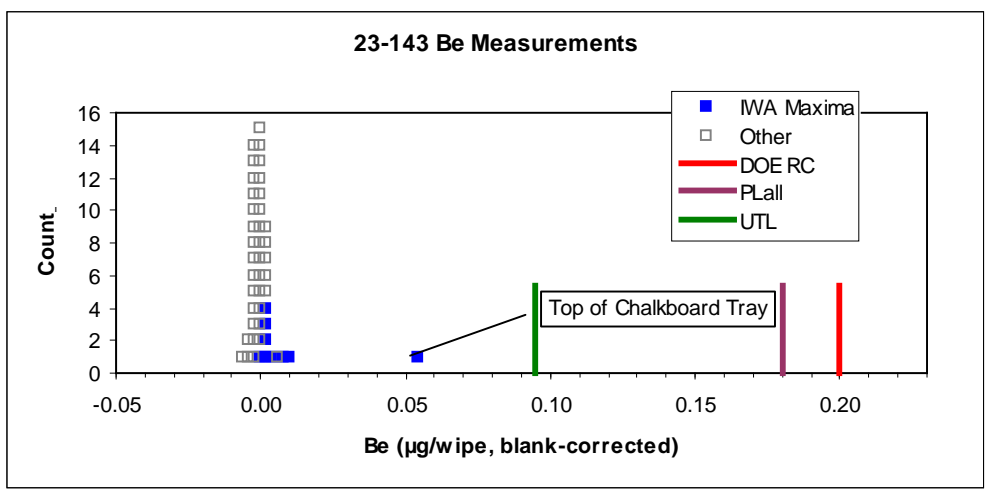

\section{3-151 \& $154 \& 156 \& 158$}

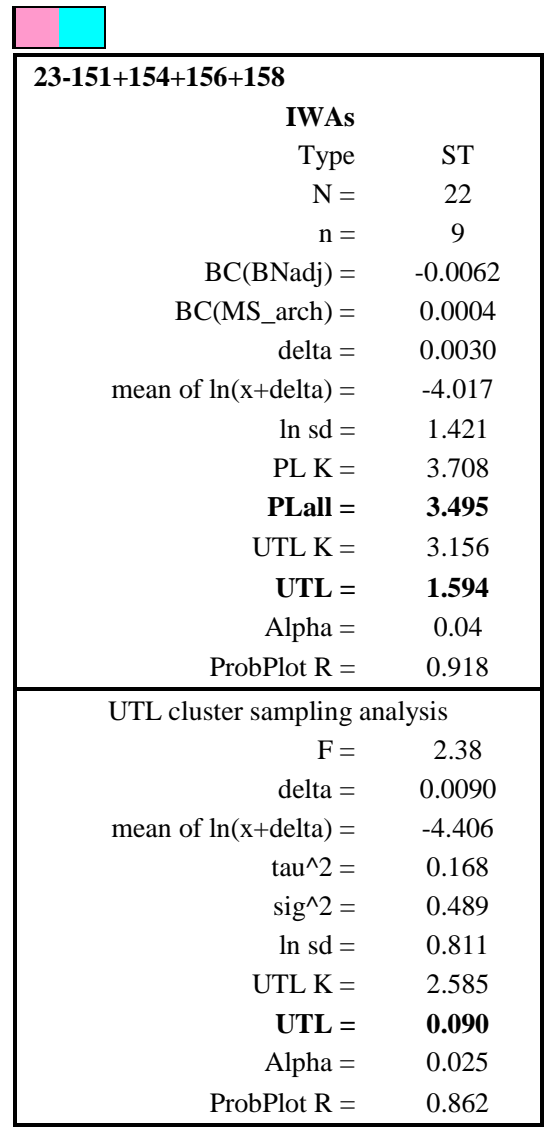

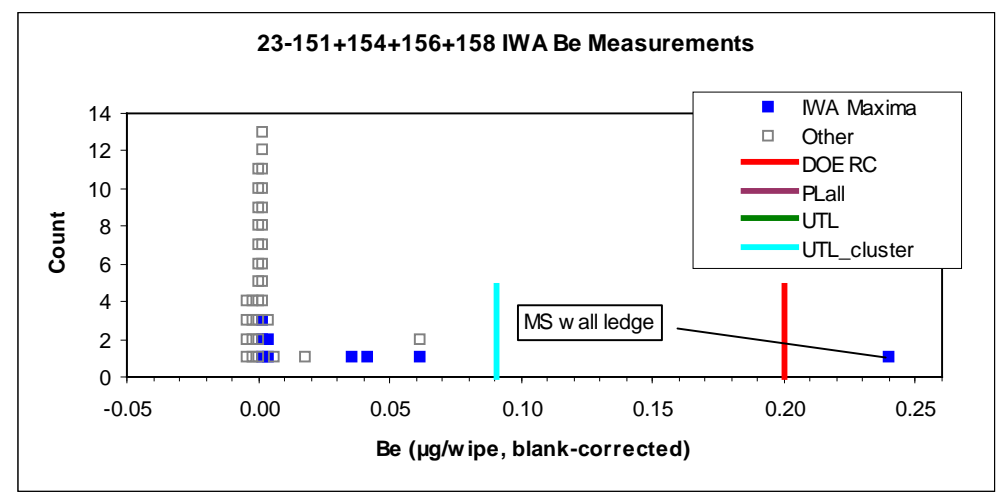

This facility is a group of connected buildings which serve as core storage at the NTS, treated as one facility for this survey.

There is one isolated MS_arch value on a wall ledge. Even including this value, UTL_cluster is well below the DOE RC. 


\begin{tabular}{|c|c|}
\hline \multicolumn{2}{|l|}{$23-151+154+156+158$} \\
\hline Area & \\
\hline Type & ST \\
\hline $\mathrm{n}=$ & 62 \\
\hline $\mathrm{BC}(\mathrm{BNadj})=$ & -0.0062 \\
\hline $\mathrm{BC}\left(\mathrm{MS} \_\right.$new $)=$ & 0.0001 \\
\hline delta $=$ & 0.0085 \\
\hline mean of $\ln (x+$ delta $)=$ & -4.158 \\
\hline $\ln \mathrm{sd}=$ & 0.840 \\
\hline UTL K = & 2.094 \\
\hline UTL = & 0.082 \\
\hline Alpha $=$ & 0.025 \\
\hline ProbPlot $\mathrm{R}=$ & 0.922 \\
\hline
\end{tabular}

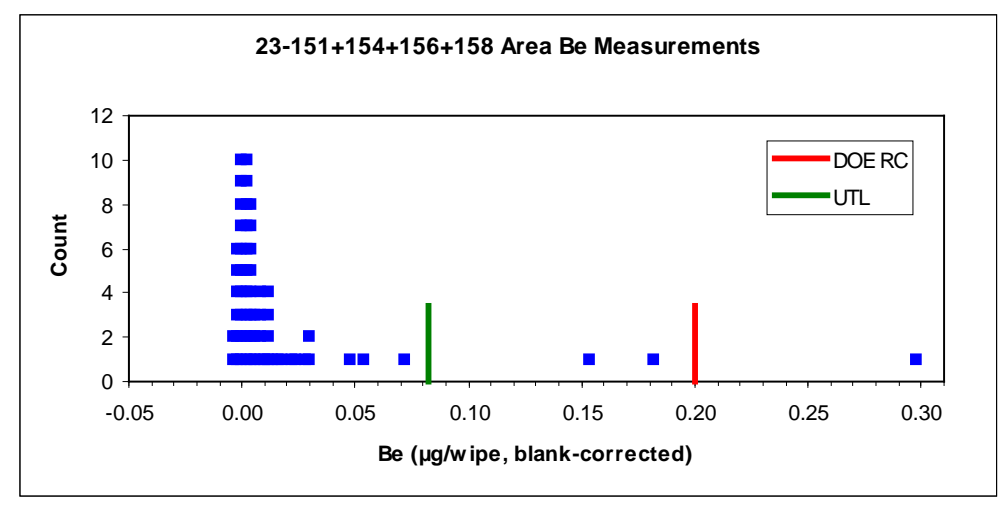

There is one MS_new value above the DOE RC. However, Be concentrations and metal ratios in six bulk samples obtained inside the facility are consistent with NTS soils, as are the metal ratios in the MS_new analysis of the wipe with high Be.

\section{3-153}

\begin{tabular}{|c|c|}
\hline \multicolumn{2}{|l|}{ 23-153 } \\
\hline Type & LA \\
\hline $\mathrm{N}=$ & 6 \\
\hline $\mathrm{n}=$ & 5 \\
\hline $\mathrm{BC}(\mathrm{BNadj})=$ & 0.0003 \\
\hline $\mathrm{BC}(\mathrm{DC})=$ & -0.0062 \\
\hline delta $=$ & 0.0020 \\
\hline mean of $\ln (x+$ delta $)=$ & -4.809 \\
\hline $\ln \mathrm{sd}=$ & 0.655 \\
\hline PL K = & 2.556 \\
\hline PLall $=$ & 0.042 \\
\hline UTL K = & 4.485 \\
\hline UTL = & 0.152 \\
\hline Alpha $=$ & 0.04 \\
\hline ProbPlot $\mathrm{R}=$ & 0.967 \\
\hline
\end{tabular}

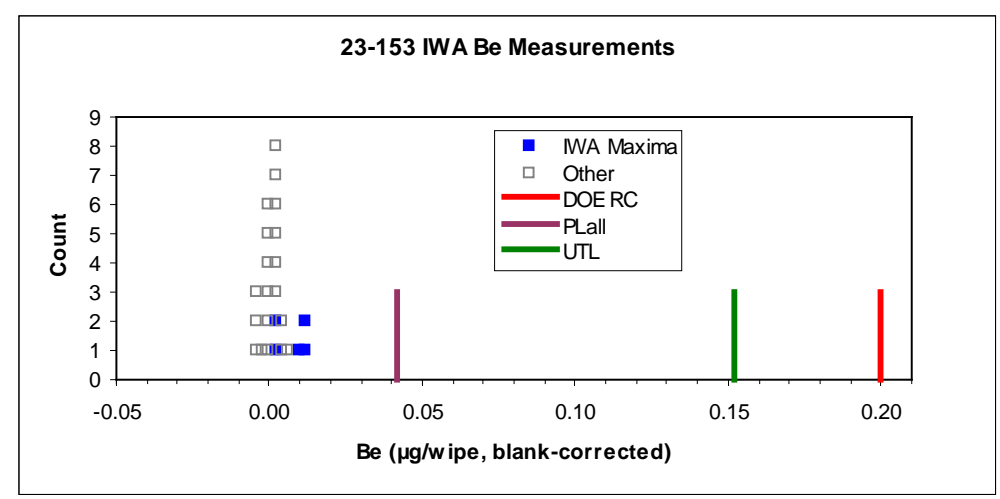

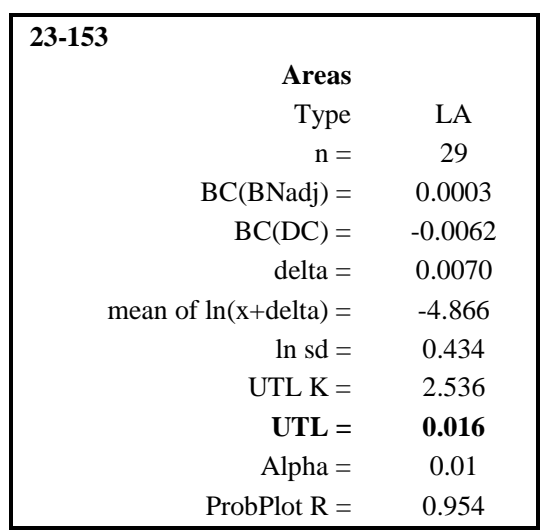

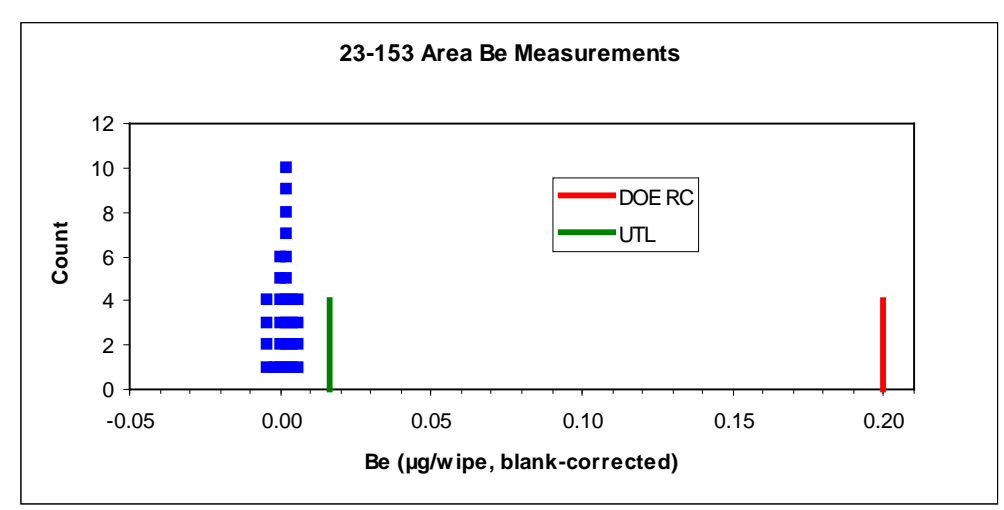




\begin{tabular}{|c|c|c|c|}
\hline $23-160$ & & & \\
\hline Worker Environment & & All Samples & \\
\hline Type & ST & & \\
\hline $\mathrm{n}=$ & 171 & $\mathrm{n}=$ & 225 \\
\hline $\mathrm{BC}(\mathrm{DC})=$ & 0.0003 & $\mathrm{BC}(\mathrm{DC})=$ & 0.0003 \\
\hline delta $=$ & 0.0055 & delta $=$ & 0.0055 \\
\hline mean of $\ln (x+$ delta $)=$ & -4.697 & mean of $\ln (x+$ delta $)=$ & -4.692 \\
\hline $\ln s d=$ & 0.452 & $\ln \mathrm{sd}=$ & 0.406 \\
\hline UTL K = & 1.848 & UTL K = & 1.821 \\
\hline UTL $=$ & 0.016 & UTL $=$ & 0.014 \\
\hline Alpha = & 0.05 & Alpha $=$ & 0.05 \\
\hline ProbPlot $\mathrm{R}=$ & 0.986 & ProbPlot $\mathrm{R}=$ & 0.980 \\
\hline
\end{tabular}

This is the surplus property warehouse in Mercury. Systematic sampling was conducted of the worker environment; in addition, samples were taken from specific designated surplus property streams for another purpose. Analyses of the worker environment data or of the combined data come to the same conclusion.

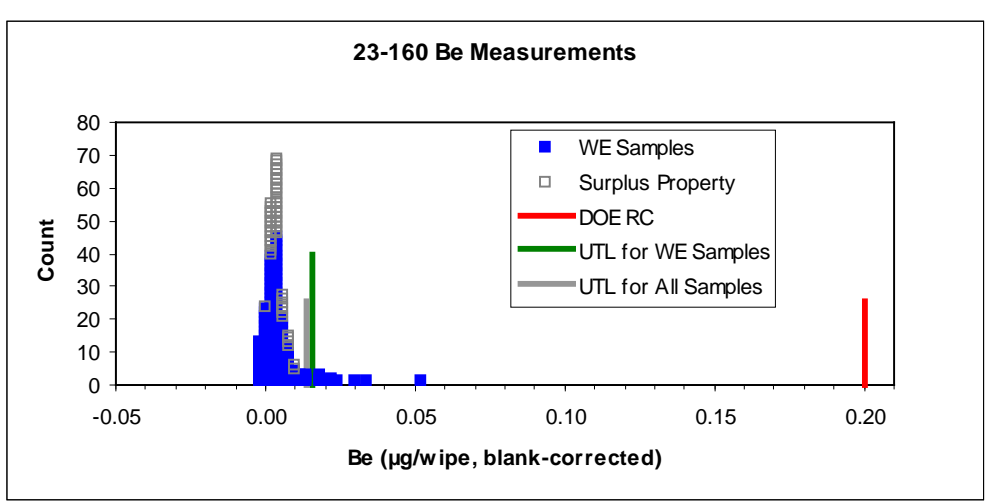

\section{3-180}

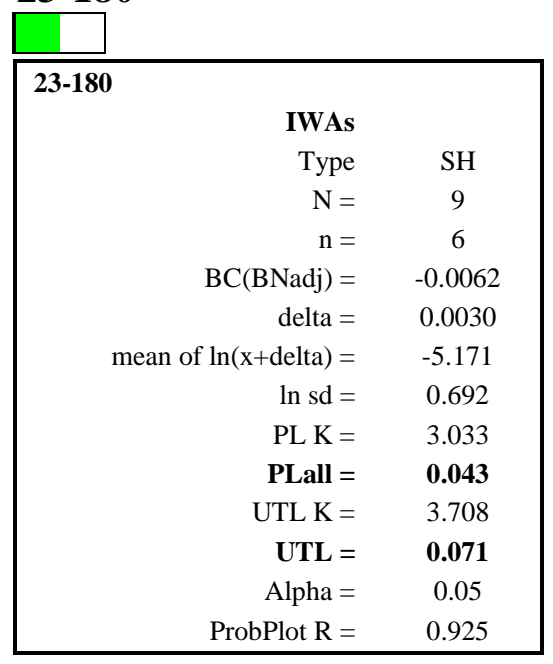

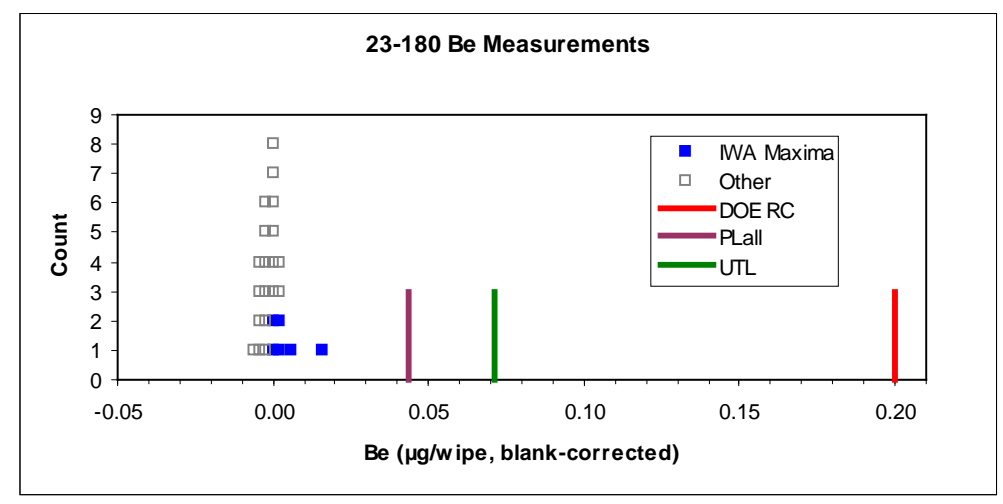




\section{3-190}

\begin{tabular}{|c|c|c|}
\hline \multirow{2}{*}{\multicolumn{3}{|c|}{ 23-190 }} \\
\hline & & \\
\hline & Type & $\mathrm{FO}$ \\
\hline & $\mathrm{N}=$ & 19 \\
\hline & $\mathrm{n}=$ & 9 \\
\hline & $\mathrm{BC}(\mathrm{DC})=$ & 0.0003 \\
\hline & delta $=$ & 0.0030 \\
\hline & mean of $\ln (x+$ delta $)=$ & -4.364 \\
\hline & $\ln \mathrm{sd}=$ & 0.267 \\
\hline & $\mathrm{PL} \mathrm{K}=$ & 3.549 \\
\hline & PLall = & 0.030 \\
\hline & UTL K = & 3.156 \\
\hline & UTL = & 0.027 \\
\hline & Alpha = & 0.04 \\
\hline & ProbPlot $\mathrm{R}=$ & 0.961 \\
\hline
\end{tabular}

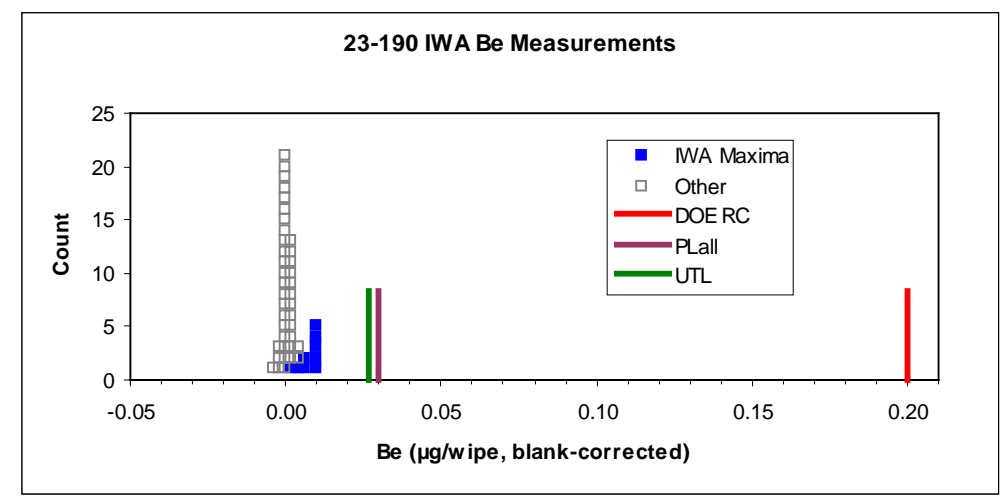

\begin{tabular}{|c|c|}
\hline \multicolumn{2}{|l|}{$23-190$} \\
\hline Type & FO \\
\hline $\mathrm{n}=$ & 93 \\
\hline NPUTL $=$ & 0.210 \\
\hline \multicolumn{2}{|c|}{ Excluding Rooms 19, 193} \\
\hline $\mathrm{n}=$ & 82 \\
\hline $\mathrm{BC}(\mathrm{DC})=$ & 0.0003 \\
\hline delta $=$ & 0.0055 \\
\hline mean of $\ln (x+$ delta $)=$ & -4.775 \\
\hline $\ln \mathrm{sd}=$ & 0.776 \\
\hline UTL $\mathrm{K}=$ & 2.107 \\
\hline UTL = & 0.038 \\
\hline Alpha $=$ & 0.01 \\
\hline ProbPlot $\mathrm{R}=$ & 0.942 \\
\hline
\end{tabular}

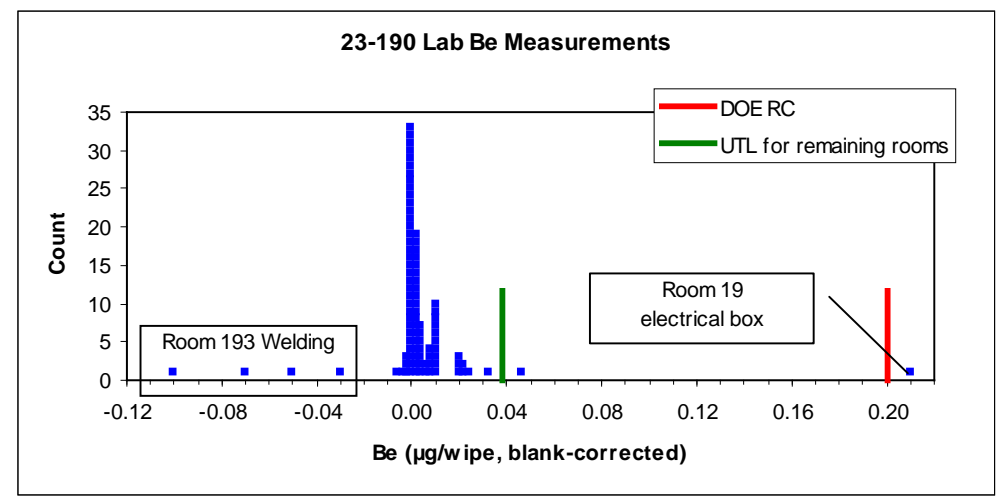

This facility contains offices and various geotechnical laboratories. Lab Room 19, used for cutting soil cores, has a value (DC) above the DOE RC. Room 193 is a welding shop. Four of five measurements obtained there were extremely negative. These two rooms are omitted from the statistical analysis presented above. They were resampled during January 2009, with 2 of 12 wipe values exceeding the DOE RC (one in each room). However, the Be concentrations and metal ratios in all four bulk samples were consistent with those of NTS soils, as were the metal ratios in the two high wipe samples. 


\section{3-211}

\begin{tabular}{|c|c|}
\hline \multirow{2}{*}{\multicolumn{2}{|c|}{ 23-211 }} \\
\hline & \\
\hline Type & FO \\
\hline $\mathrm{N}=$ & 14 \\
\hline $\mathrm{n}=$ & 6 \\
\hline $\mathrm{BC}(\mathrm{BNadj})=$ & -0.0062 \\
\hline $\mathrm{BC}(\mathrm{MS})=$ & 0.0004 \\
\hline delta $=$ & 0.0030 \\
\hline mean of $\ln (x+$ delta $)=$ & -5.051 \\
\hline $\ln \mathrm{sd}=$ & 0.217 \\
\hline PL K = & 4.039 \\
\hline PLall $=$ & 0.012 \\
\hline UTL K = & 3.918 \\
\hline UTL = & 0.012 \\
\hline Alpha $=$ & 0.04 \\
\hline ProbPlot $\mathrm{R}=$ & 0.977 \\
\hline
\end{tabular}

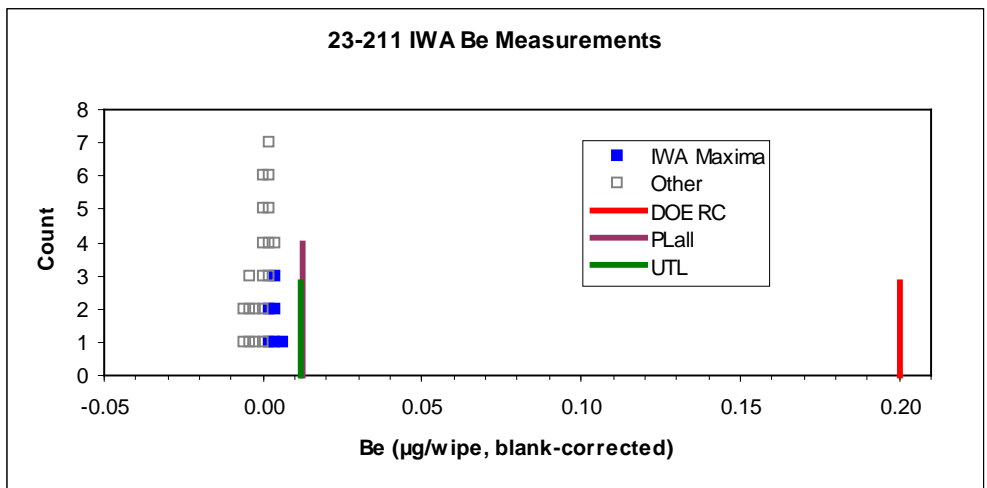

\begin{tabular}{rc|} 
23-211 & \\
Type & FO \\
$\mathrm{n}=$ & 46 \\
BC(BNadj $)=$ & -0.0062 \\
BC $(\mathrm{MS})=$ & 0.0004 \\
delta $=$ & 0.0080 \\
mean of $\ln (\mathrm{x}+$ delta $)=$ & -4.937 \\
$\ln \mathrm{sd}=$ & 0.440 \\
$\mathrm{UTL} \mathrm{K}=$ & 2.303 \\
$\mathrm{UTL}=$ & $\mathbf{0 . 0 1 2}$ \\
Alpha $=$ & 0.01 \\
ProbPlot $\mathrm{R}=$ & 0.970 \\
\hline
\end{tabular}

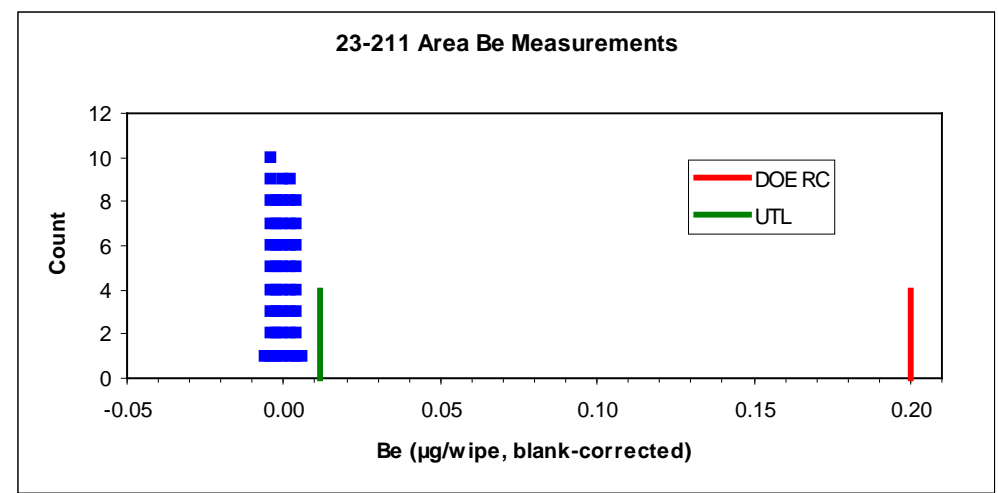

\section{3-300}

\begin{tabular}{|c|c|}
\hline \multirow{2}{*}{\multicolumn{2}{|c|}{ 23-300 }} \\
\hline & \\
\hline Type & QU \\
\hline $\mathrm{N}=$ & 22 \\
\hline $\mathrm{n}=$ & 8 \\
\hline $\mathrm{BC}(\mathrm{DC})=$ & 0.0003 \\
\hline delta $=$ & 0.0050 \\
\hline mean of $\ln (x+$ delta $)=$ & -4.846 \\
\hline $\ln \mathrm{sd}=$ & 0.436 \\
\hline PL K = & 3.916 \\
\hline PLall = & 0.038 \\
\hline UTL K = & 3.331 \\
\hline UTL $=$ & 0.029 \\
\hline Alpha $=$ & 0.04 \\
\hline ProbPlot $\mathrm{R}=$ & 0.934 \\
\hline
\end{tabular}

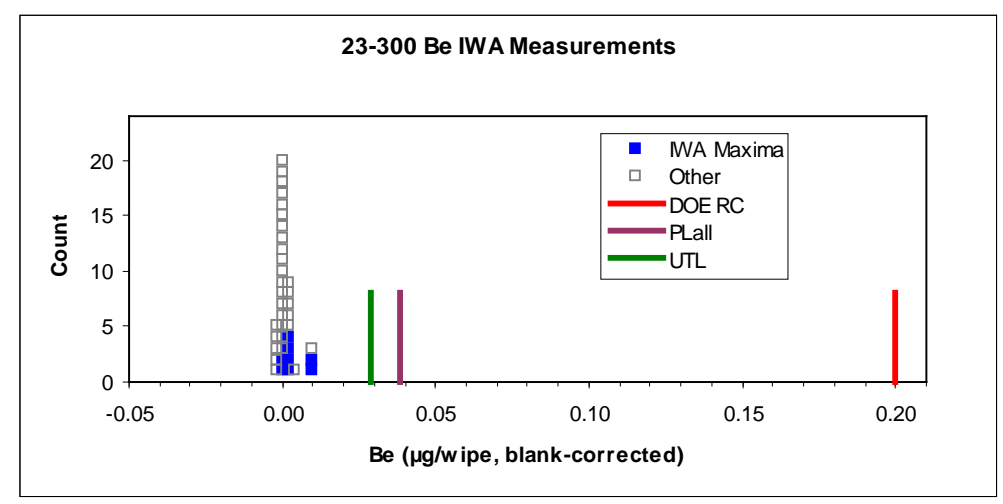




\begin{tabular}{|c|c|}
\hline \multicolumn{2}{|l|}{ 23-300 } \\
\hline Areas & \\
\hline Type & QU \\
\hline $\mathrm{n}=$ & 112 \\
\hline shift $=$ & 0.0003 \\
\hline delta $=$ & 0.0020 \\
\hline mean of $\ln (x+$ delta $)=$ & -5.433 \\
\hline $\ln \mathrm{sd}=$ & 0.765 \\
\hline UTL $\mathrm{K}=$ & 2.030 \\
\hline UTL = & 0.019 \\
\hline Alpha $=$ & 0.01 \\
\hline ProbPlot $\mathrm{R}=$ & 0.944 \\
\hline
\end{tabular}

\section{3-310}

\begin{tabular}{|c|c|}
\hline \multicolumn{2}{|l|}{ 23-310 } \\
\hline IWAs & \\
\hline Туре & OF \\
\hline $\mathrm{N}=$ & 33 \\
\hline $\mathrm{n}=$ & 9 \\
\hline $\mathrm{BC}(\mathrm{BNadj})=$ & -0.0062 \\
\hline BC(MS_arch $)=$ & 0.0004 \\
\hline delta $=$ & 0.0040 \\
\hline mean of $\ln (x+$ delta $)=$ & -5.169 \\
\hline $\ln \mathrm{sd}=$ & 0.546 \\
\hline PL K = & 3.894 \\
\hline PLall $=$ & 0.044 \\
\hline UTL K = & 3.031 \\
\hline UTL $=$ & 0.026 \\
\hline Alpha $=$ & 0.05 \\
\hline ProbPlot $\mathrm{R}=$ & 0.833 \\
\hline
\end{tabular}

\section{3-425}

$\square$

\begin{tabular}{|c|c|}
\hline \multicolumn{2}{|l|}{$23-425$} \\
\hline IWAs & \\
\hline Type & FO \\
\hline $\mathrm{N}=$ & 50 \\
\hline $\mathrm{n}=$ & 30 \\
\hline $\mathrm{BC}(\mathrm{BNadj})=$ & -0.0062 \\
\hline $\mathrm{BC}(\mathrm{DC})=$ & 0.0013 \\
\hline BC(MS_arch) $=$ & 0.0004 \\
\hline delta $=$ & 0.0030 \\
\hline mean of $\ln (x+$ delta $)=$ & -4.600 \\
\hline $\ln \mathrm{sd}=$ & 1.067 \\
\hline PL $K=$ & 3.055 \\
\hline PLall $=$ & 0.259 \\
\hline UTL K = & 2.220 \\
\hline $\mathbf{U T L}=$ & 0.104 \\
\hline Alpha $=$ & 0.05 \\
\hline ProbPlot $\mathrm{R}=$ & 0.978 \\
\hline
\end{tabular}
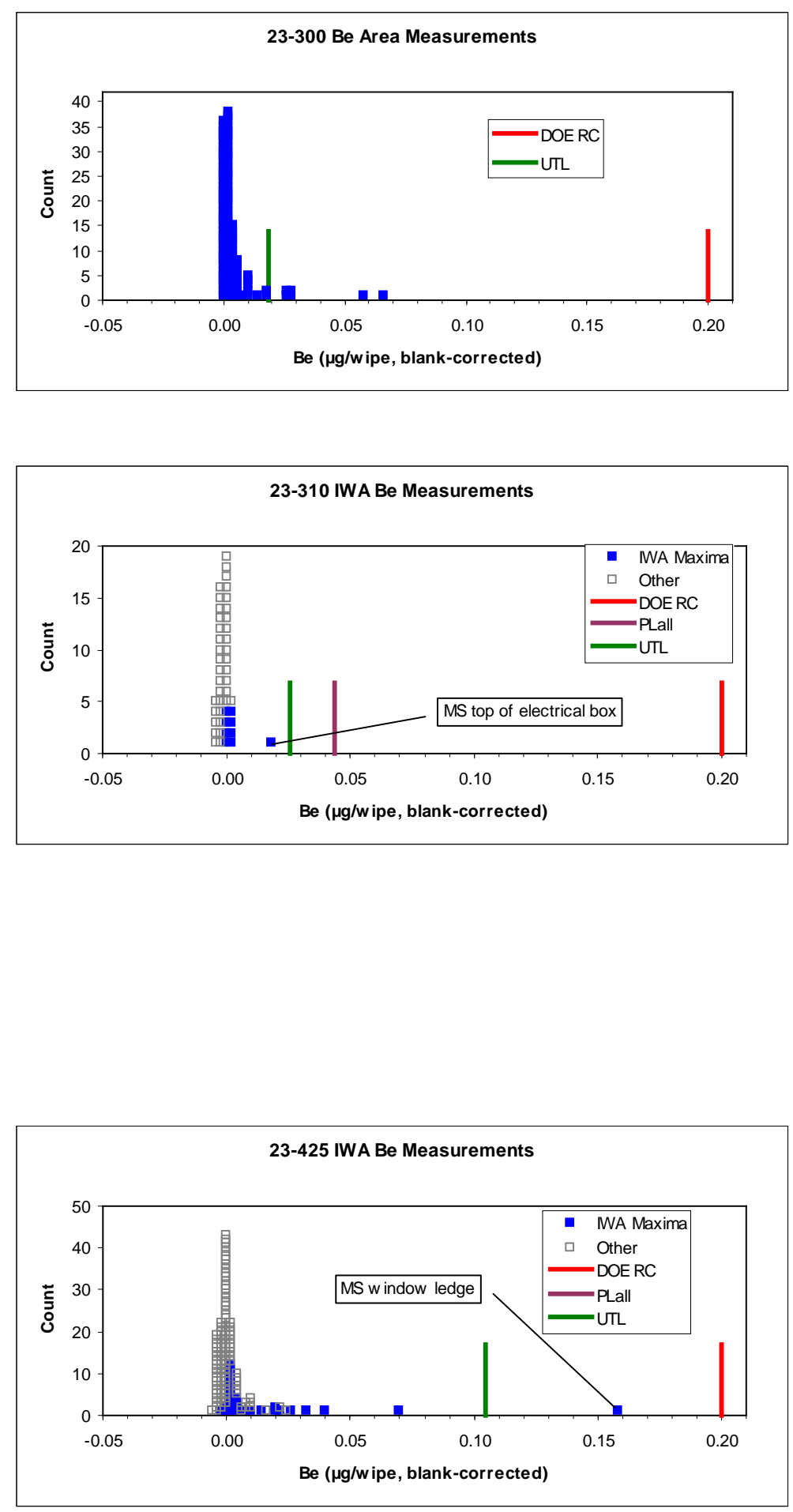


\begin{tabular}{|c|c|}
\hline \multirow{2}{*}{\multicolumn{2}{|c|}{ 23-475 etc. }} \\
\hline & \\
\hline Туре & QU \\
\hline $\mathrm{N}=$ & 87 \\
\hline $\mathrm{n}=$ & 14 \\
\hline $\mathrm{BC}(\mathrm{BNadj})=$ & -0.0062 \\
\hline BC(MS_arch $)=$ & 0.0004 \\
\hline delta $=$ & 0.004 \\
\hline mean of $\ln (x+$ delta $)=$ & -4.200 \\
\hline $\ln \mathrm{sd}=$ & 1.069 \\
\hline PL K = & 3.985 \\
\hline PLall $=$ & 1.057 \\
\hline UTL K = & 2.614 \\
\hline UTL = & 0.241 \\
\hline Alpha $=$ & 0.05 \\
\hline ProbPlot $\mathrm{R}=$ & 0.931 \\
\hline \multicolumn{2}{|c|}{ omitting door tops } \\
\hline mean of $\ln (x+$ delta $)=$ & -4.845 \\
\hline $\ln \mathrm{sd}=$ & 0.409 \\
\hline PLall $=$ & 0.036 \\
\hline UTL $=$ & 0.019 \\
\hline ProbPlot $\mathrm{R}=$ & 0.930 \\
\hline \multicolumn{2}{|c|}{ UTL cluster sampling analysis } \\
\hline $\mathrm{F}=$ & 0.87 \\
\hline delta $=$ & 0.0085 \\
\hline mean of $\ln (x+$ delta $)=$ & -4.612 \\
\hline $\ln \mathrm{sd}=$ & 0.631 \\
\hline UTL K = & 1.984 \\
\hline UTL = & 0.026 \\
\hline ProbPlot $\mathrm{R}=$ & 0.916 \\
\hline
\end{tabular}

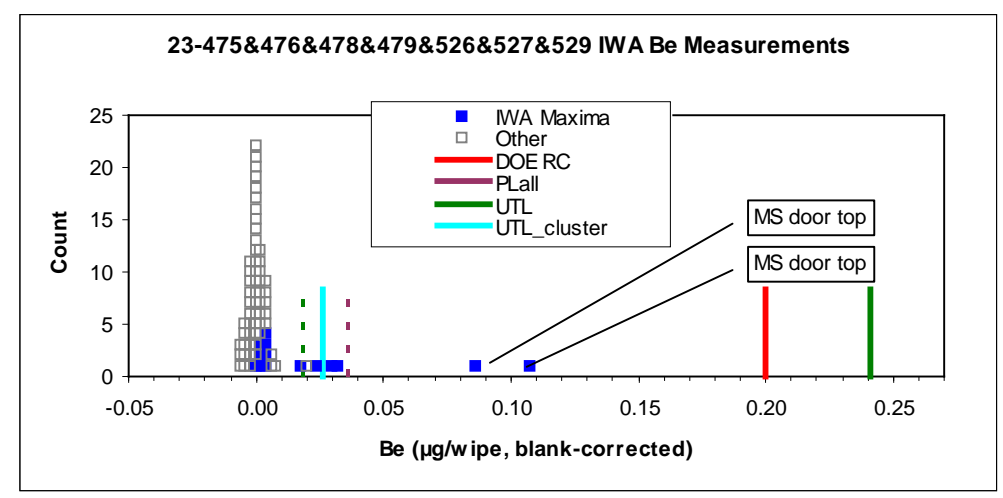

This facility is a group of adjacent dormitories with very similar attributes, treated as one unit for this survey. The two somewhat elevated MS_arch door top values push PLall and UTL over the DOE RC. When these are omitted, or when the UTL cluster analysis is performed, which uses all data rather than just the IWA maxima, the facility "passes."

\section{$23-480 \& 481 \& 482 \& 483 \& 484$}

\begin{tabular}{|c|c|}
\hline \multirow{2}{*}{\multicolumn{2}{|c|}{ 23-480 through 484}} \\
\hline & \\
\hline Type & QU \\
\hline $\mathrm{N}=$ & 41 \\
\hline $\mathrm{n}=$ & 9 \\
\hline $\mathrm{BC}(\mathrm{DC})=$ & 0.0013 \\
\hline delta $=$ & 0.005 \\
\hline mean of $\ln (x+$ delta $)=$ & -3.961 \\
\hline $\ln \mathrm{sd}=$ & 0.354 \\
\hline PL K = & 4.057 \\
\hline PLall = & 0.075 \\
\hline UTL K = & 3.031 \\
\hline UTL $=$ & 0.051 \\
\hline Alpha $=$ & 0.05 \\
\hline ProbPlot $\mathrm{R}=$ & 0.961 \\
\hline
\end{tabular}

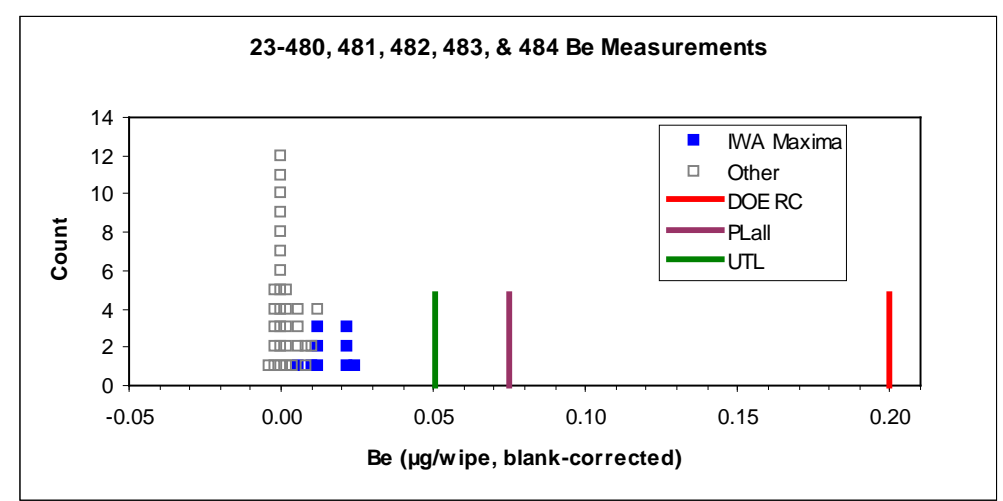

This facility consists of four contiguous dormitories and their associated dayroom. 
23-525

$\begin{array}{rc}\text { IWAs } & \\ \text { Type } & \text { OF } \\ \mathrm{N}= & 27 \\ \mathrm{n}= & 8 \\ \mathrm{BC}(\mathrm{BNadj})= & -0.0062 \\ \mathrm{BC}(\mathrm{MS})= & 0.0004 \\ \text { delta }= & 0.0040 \\ \text { mean of } \ln (\mathrm{x}+\text { delta })= & -4.476 \\ \ln \mathrm{sd}= & 0.577 \\ \mathrm{PL} \mathrm{K}= & 3.917 \\ \text { PLall }= & \mathbf{0 . 1 0 5} \\ \text { UTL K }= & 3.187 \\ \text { UTL }= & \mathbf{0 . 0 6 7} \\ \text { Alpha }= & 0.05 \\ \text { ProbPlot } \mathrm{R}= & 0.931\end{array}$

\section{$23-528 \& 530$}

\begin{tabular}{|c|c|}
\hline \multicolumn{2}{|l|}{$23-528 \& 530$} \\
\hline IWAs & \\
\hline Type & QU \\
\hline $\mathrm{N}=$ & 86 \\
\hline $\mathrm{n}=$ & 10 \\
\hline $\mathrm{BC}(\mathrm{DC})=$ & 0.0013 \\
\hline delta $=$ & 0.0050 \\
\hline mean of $\ln (x+$ delta $)=$ & -3.002 \\
\hline $\ln \mathrm{sd}=$ & 0.289 \\
\hline $\mathrm{PL} \mathrm{K}=$ & 4.375 \\
\hline PLall $=$ & 0.171 \\
\hline UTL K = & 2.911 \\
\hline UTL = & 0.110 \\
\hline Alpha $=$ & 0.05 \\
\hline ProbPlot $\mathrm{R}=$ & 0.988 \\
\hline
\end{tabular}

\section{3-531 \& $532 \& 535$}

\begin{tabular}{|c|c|}
\hline \multicolumn{2}{|l|}{$23-531 \& 532 \& 535$} \\
\hline IWAs & \\
\hline Туре & QU \\
\hline $\mathrm{N}=$ & 159 \\
\hline $\mathrm{n}=$ & 15 \\
\hline $\mathrm{BC}(\mathrm{BNadj})=$ & -0.0062 \\
\hline delta $=$ & 0.0050 \\
\hline mean of $\ln (x+$ delta $)=$ & -4.965 \\
\hline $\ln \mathrm{sd}=$ & 0.192 \\
\hline PL K = & 4.218 \\
\hline PLall $=$ & 0.011 \\
\hline UTL K = & 2.566 \\
\hline UTL $=$ & 0.006 \\
\hline Alpha $=$ & 0.05 \\
\hline ProbPlot $\mathrm{R}=$ & 0.949 \\
\hline
\end{tabular}

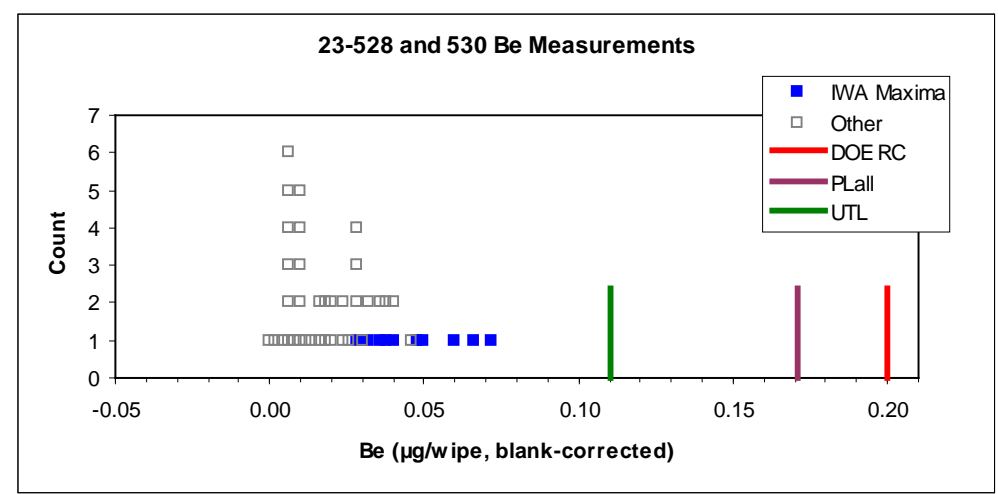

This facility consists of two adjacent similar dormitories.

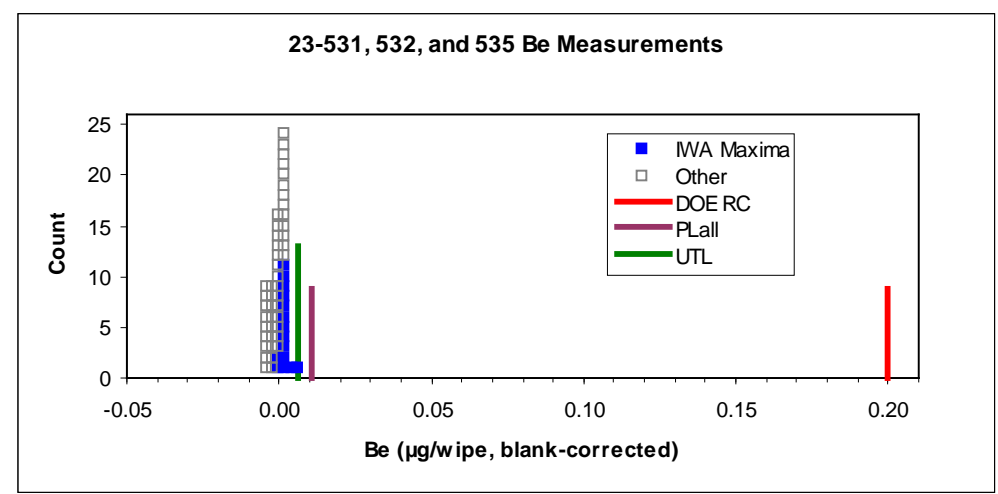

This facility consists of three adjacent similar dormitories.

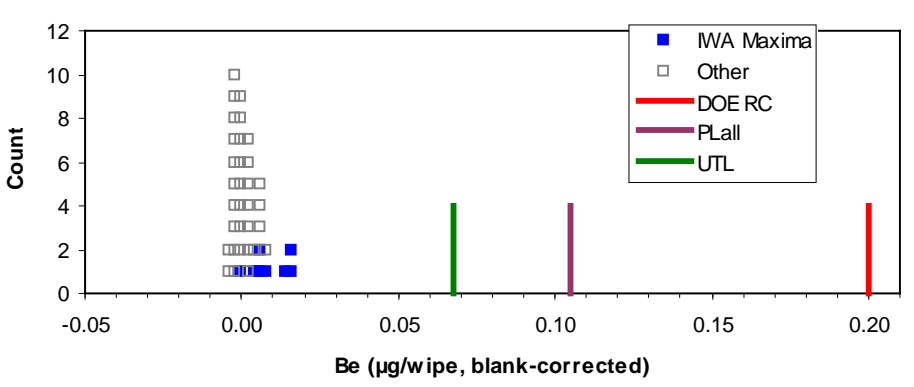


23-550

$\square$

23-550

$\begin{array}{rc}\text { IWAs } & \\ \text { Type } & \text { OF } \\ \mathrm{N}= & 23 \\ \mathrm{n}= & 8 \\ \mathrm{BC}(\mathrm{DC})= & 0.0003 \\ \text { delta }= & 0.0050 \\ \text { mean of } \ln (\mathrm{x}+\text { delta })= & -4.675 \\ \ln \mathrm{sd}= & 0.336 \\ \mathrm{PL} \mathrm{K}= & 3.771 \\ \text { PLall }= & \mathbf{0 . 0 2 8} \\ \text { UTL } \mathrm{K}= & 3.187 \\ \text { UTL }= & \mathbf{0 . 0 2 2} \\ \text { Alpha }= & 0.05 \\ \text { ProbPlot } \mathrm{R}= & 0.967\end{array}$

\section{3-600}

\begin{tabular}{|c|c|c|}
\hline \multirow{2}{*}{\multicolumn{3}{|c|}{$23-600$}} \\
\hline & & \\
\hline & Tyре & EX \\
\hline & $\mathrm{N}=$ & 142 \\
\hline & $\mathrm{n}=$ & 27 \\
\hline & $\mathrm{BC}(\mathrm{DC})=$ & 0.0003 \\
\hline & delta $=$ & 0.0020 \\
\hline & mean of $\ln (x+$ delta $)=$ & -5.449 \\
\hline & $\ln \mathrm{sd}=$ & 0.782 \\
\hline & PL K = & 3.847 \\
\hline & PLall $=$ & 0.085 \\
\hline & UTL K = & 2.307 \\
\hline & UTL $=$ & 0.024 \\
\hline & Alpha $=$ & 0.04 \\
\hline & ProbPlot $\mathrm{R}=$ & 0.968 \\
\hline
\end{tabular}

\begin{tabular}{|c|c|}
\hline \multicolumn{2}{|l|}{ 23-600 } \\
\hline Areas & \\
\hline Туре & EX \\
\hline $\mathrm{n}=$ & 47 \\
\hline shift $=$ & 0.0003 \\
\hline delta $=$ & 0.0070 \\
\hline mean of $\ln (x+$ delta $)=$ & -5.035 \\
\hline $\ln \mathrm{sd}=$ & 0.122 \\
\hline UTL K = & 2.294 \\
\hline UTL $=$ & 0.002 \\
\hline Alpha $=$ & 0.01 \\
\hline ProbPlot $\mathrm{R}=$ & 0.945 \\
\hline
\end{tabular}
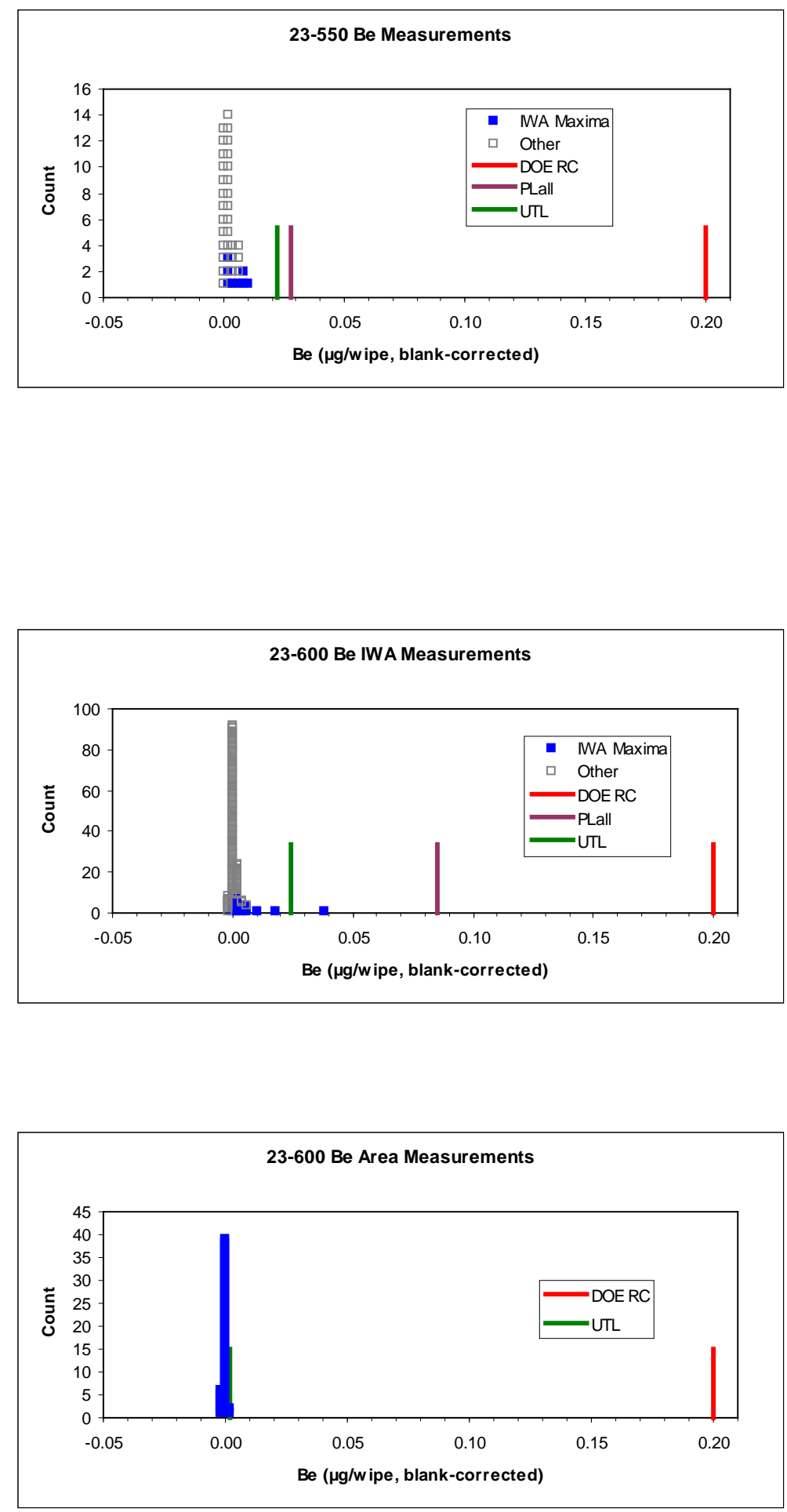
23-610

\begin{tabular}{|c|c|}
\hline \multicolumn{2}{|l|}{ 23-610 } \\
\hline \multicolumn{2}{|l|}{ Area } \\
\hline Type & FO \\
\hline $\mathrm{n}=$ & 21 \\
\hline BC $($ BNadj $)=$ & -0.0062 \\
\hline BC(MS_new) = & 0.0001 \\
\hline delta $=$ & 0.0085 \\
\hline mean of $\ln (x+$ delta $)=$ & -4.409 \\
\hline $\ln \mathrm{sd}=$ & 1.212 \\
\hline UTL K = & 2.371 \\
\hline UTL $=$ & 0.214 \\
\hline Alpha $=$ & 0.05 \\
\hline ProbPlot R = & 0.962 \\
\hline \multicolumn{2}{|l|}{ omitting windowsill } \\
\hline $\mathrm{n}=$ & 20 \\
\hline delta $=$ & 0.0085 \\
\hline mean of $\ln (x+$ delta $)=$ & -4.573 \\
\hline $\ln \mathrm{sd}=$ & 0.976 \\
\hline UTL K = & 2.396 \\
\hline UTL $=$ & 0.106 \\
\hline Alpha = & 0.05 \\
\hline ProbPlot R = & 0.976 \\
\hline
\end{tabular}

\section{3-614}

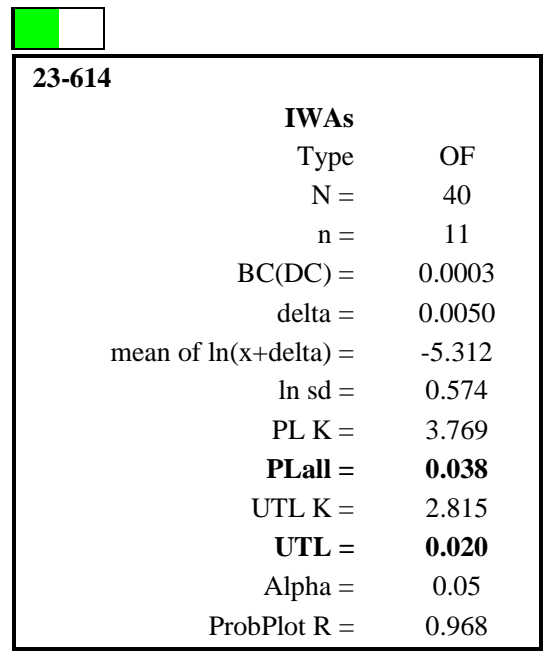

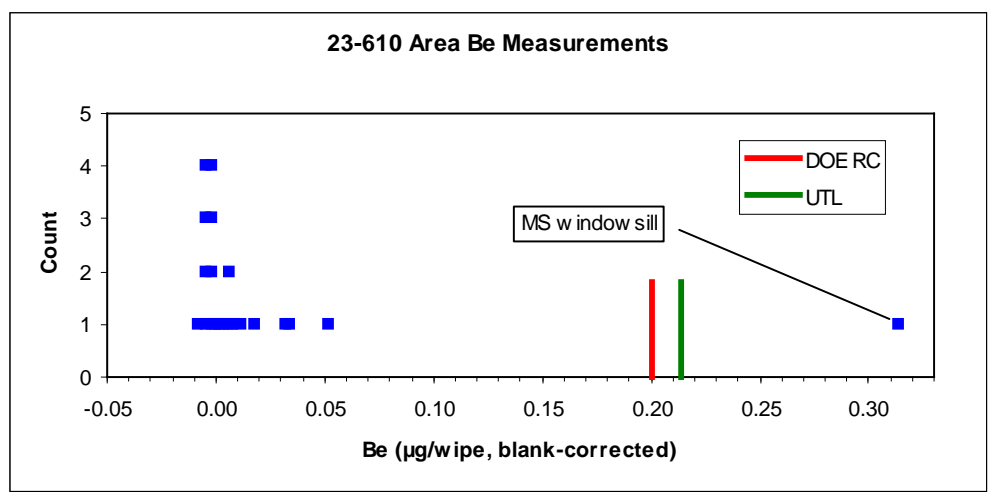

There is one isolated MS_new value on a windowsill that is above the DOE RC; omitting that, UTL is less than the RC. The bulk sample Be is low and the metal ratios are consistent with NTS soils. The metal ratios for the high wipe are consistent with NTS soils except that Ni is reported as zero.

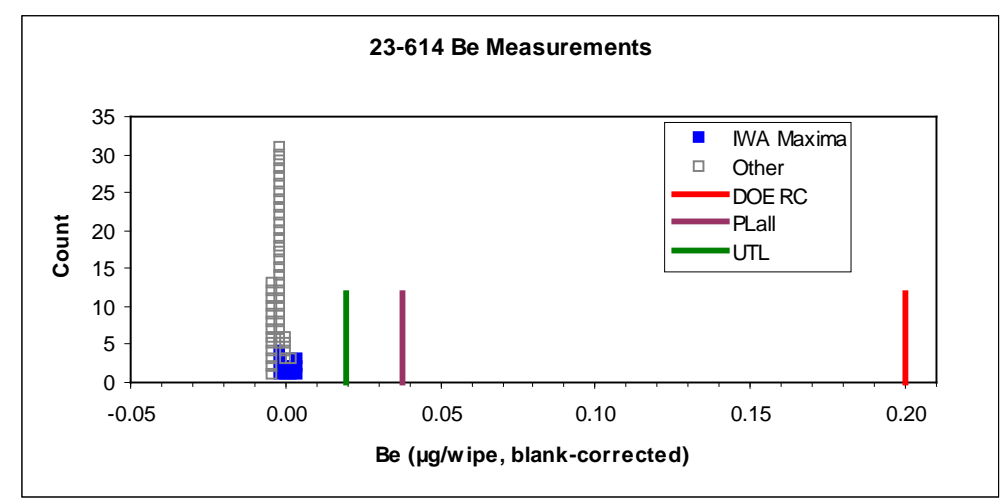




\section{3-620}

\begin{tabular}{|c|c|c|}
\hline \multicolumn{3}{|l|}{$23-620$} \\
\hline & IWAs & \\
\hline & Type & OF \\
\hline & $\mathrm{N}=$ & 12 \\
\hline & $\mathrm{n}=$ & 6 \\
\hline & $\mathrm{BC}(\mathrm{DC})=$ & 0.0003 \\
\hline & delta $=$ & 0.0050 \\
\hline & mean of $\ln (x+$ delta $)=$ & -4.552 \\
\hline & $\ln \mathrm{sd}=$ & 0.299 \\
\hline & PL K = & 3.576 \\
\hline & PLall = & 0.026 \\
\hline & UTL K = & 3.708 \\
\hline & UTL = & 0.027 \\
\hline & Alpha $=$ & 0.05 \\
\hline & ProbPlot $\mathrm{R}=$ & 0.978 \\
\hline
\end{tabular}

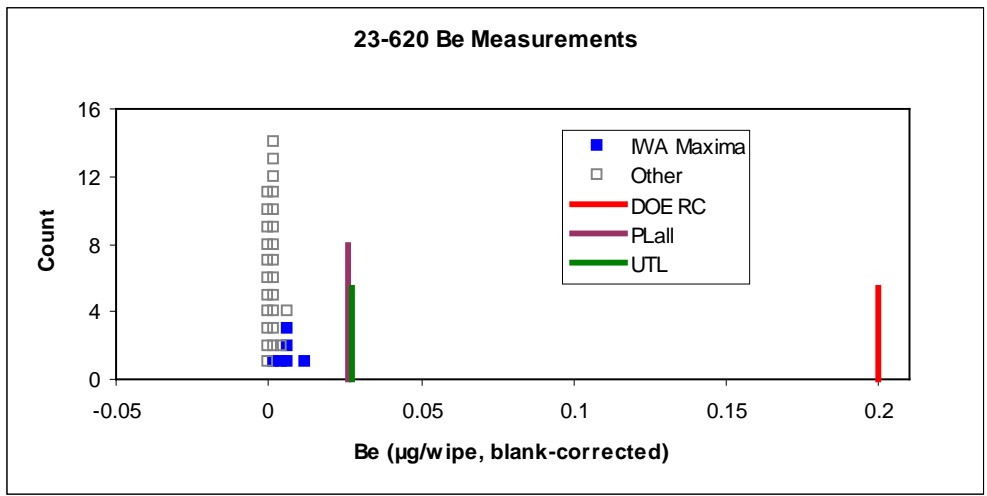

\section{3-630}

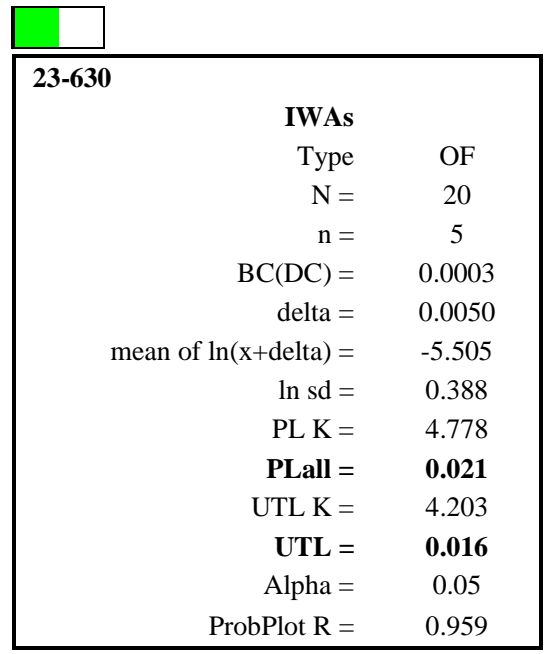

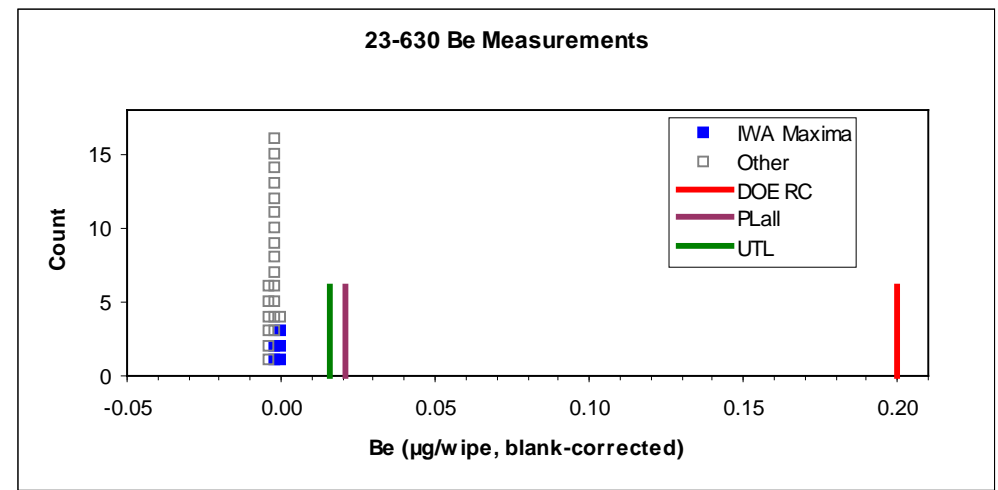

\section{3-650}

\begin{tabular}{|c|c|c|}
\hline \multicolumn{3}{|l|}{$23-650$} \\
\hline & IWAs & \\
\hline & Type & LA \\
\hline & $\mathrm{N}=$ & 139 \\
\hline & $\mathrm{n}=$ & 23 \\
\hline & $\mathrm{BC}(\mathrm{DC})=$ & 0.0003 \\
\hline & delta $=$ & 0.002 \\
\hline & mean of $\ln (x+$ delta $)=$ & -5.546 \\
\hline & $\ln \mathrm{sd}=$ & 0.286 \\
\hline & PL K = & 3.833 \\
\hline & PLall = & 0.010 \\
\hline & UTL K = & 2.328 \\
\hline & UTL = & 0.006 \\
\hline & Alpha $=$ & 0.05 \\
\hline & ProbPlot $\mathrm{R}=$ & 0.959 \\
\hline
\end{tabular}

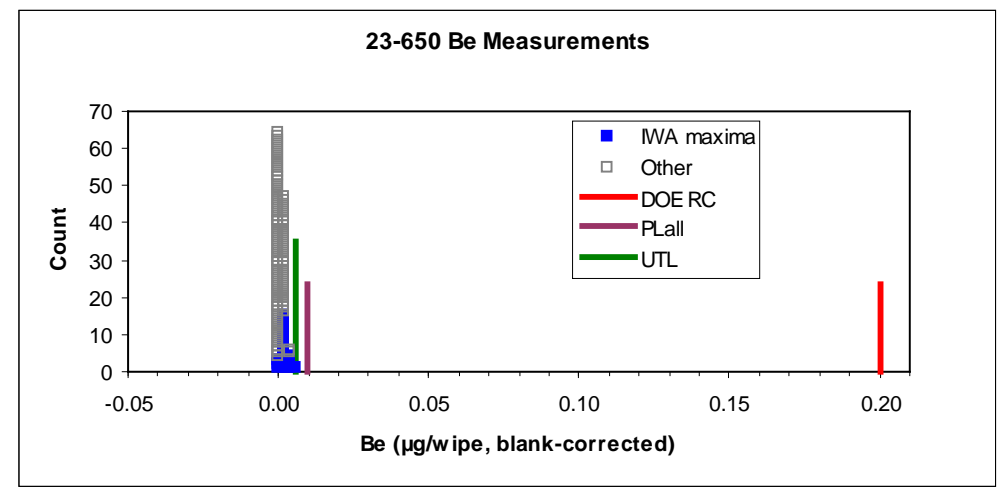




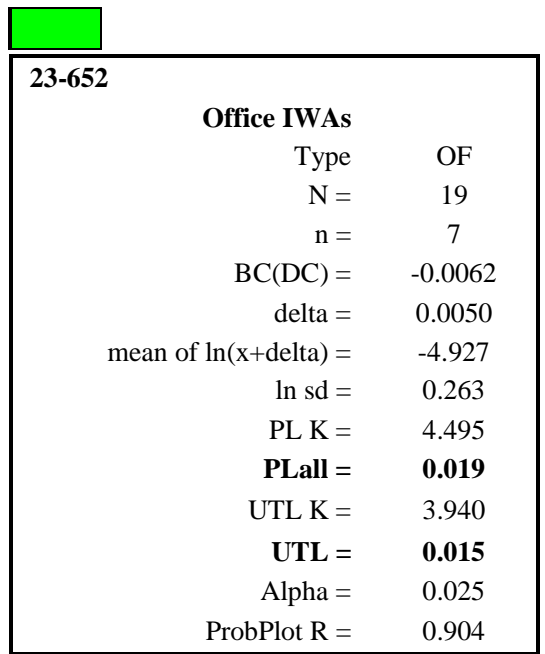

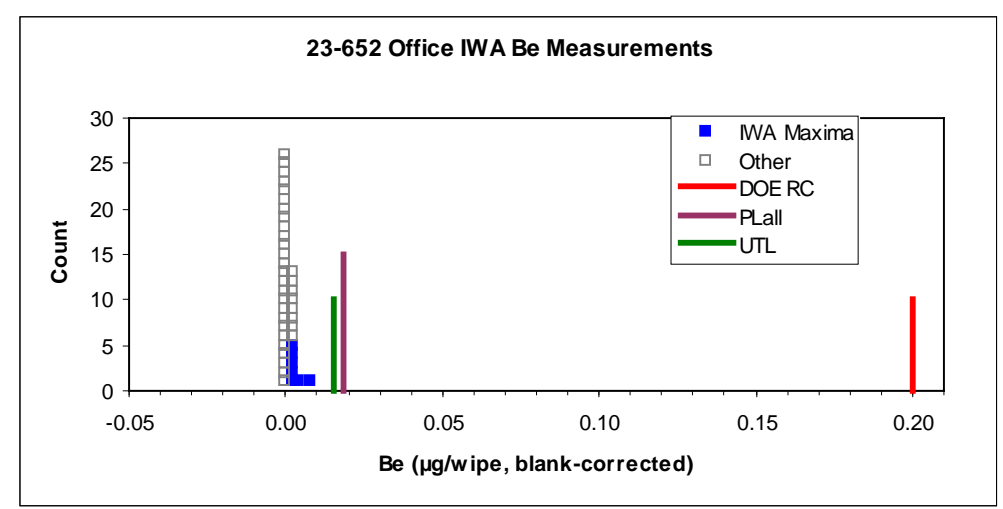

This facility consists of two distinct areas: offices and laboratories. With the initial sampling, the offices were fine, but the laboratory areas had a different and somewhat higher distribution of Be measurements. The lab IWAs were resampled so that wipes were taken from the dustiest corners in each designated lab IWA. The highest wipe obtained in the laboratories was $0.044 \mu \mathrm{g} / \mathrm{wipe}$ (DC).

\section{$23-675 \& 676 \& 678 \& 679 \& 680 \& 681 \& 683 \& 684$}

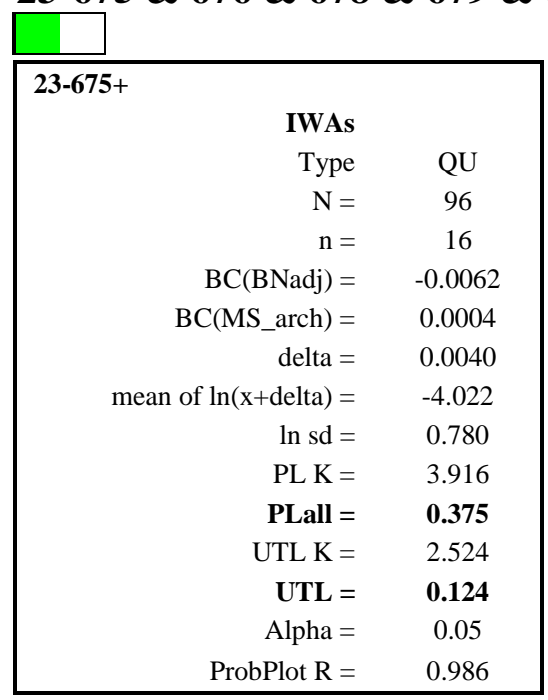

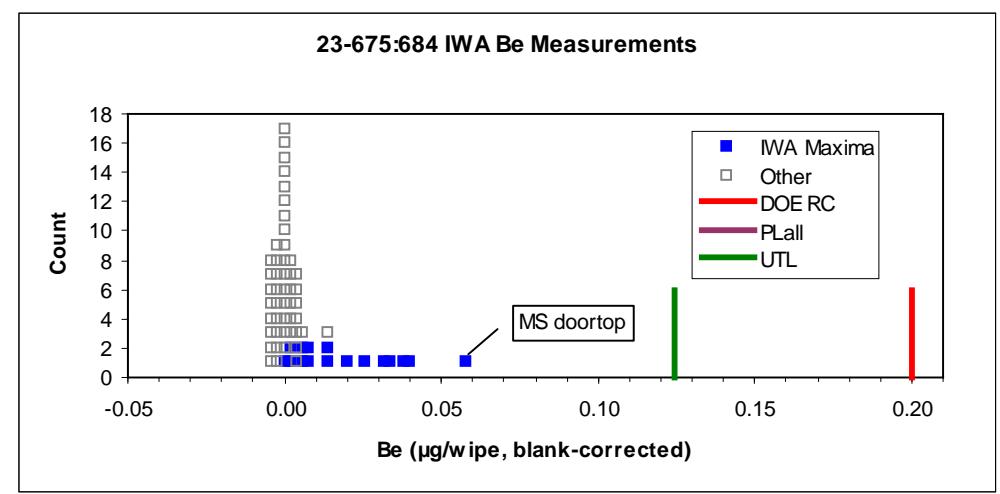

This facility consists of adjacent dormitories. 
23-700

\begin{tabular}{|c|c|}
\hline \multicolumn{2}{|l|}{$23-700$} \\
\hline IWAs & \\
\hline Type & $\mathrm{SH}$ \\
\hline $\mathrm{N}=$ & 9 \\
\hline $\mathrm{n}=$ & 4 \\
\hline $\mathrm{BC}(\mathrm{DC})=$ & 0.0003 \\
\hline delta $=$ & 0.0030 \\
\hline mean of $\ln (x+$ delta $)=$ & -5.101 \\
\hline $\ln s d=$ & 0.536 \\
\hline $\mathrm{PL} \mathrm{K}=$ & 4.472 \\
\hline PLall = & 0.064 \\
\hline UTL K = & 5.580 \\
\hline UTL = & 0.118 \\
\hline Alpha $=$ & 0.04 \\
\hline ProbPlot $\mathrm{R}=$ & 0.960 \\
\hline
\end{tabular}

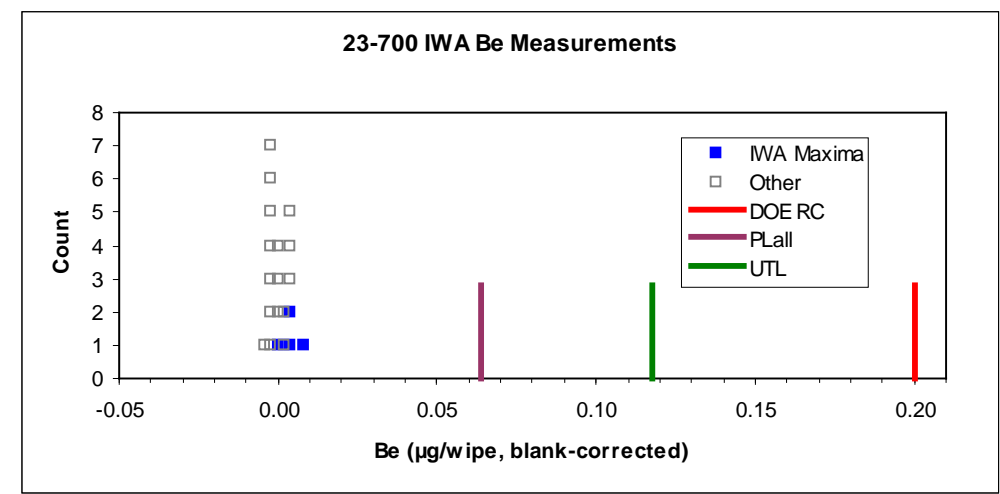

\begin{tabular}{|c|c|}
\hline \multicolumn{2}{|l|}{ 23-700 } \\
\hline Area & \\
\hline Туре & $\mathrm{SH}$ \\
\hline $\mathrm{n}=$ & 66 \\
\hline $\mathrm{BC}(\mathrm{BNadj})=$ & -0.0062 \\
\hline $\mathrm{BC}(\mathrm{DC})=$ & 0.0013 \\
\hline BC(MS_arch $)=$ & 0.0004 \\
\hline delta $=$ & 0.0080 \\
\hline mean of $\ln (x+$ delta $)=$ & -4.488 \\
\hline $\ln s d=$ & 0.685 \\
\hline UTL K = & 2.171 \\
\hline $\mathbf{U T L}=$ & 0.011 \\
\hline Alpha $=$ & 0.01 \\
\hline ProbPlot $\mathrm{R}=$ & 0.964 \\
\hline
\end{tabular}

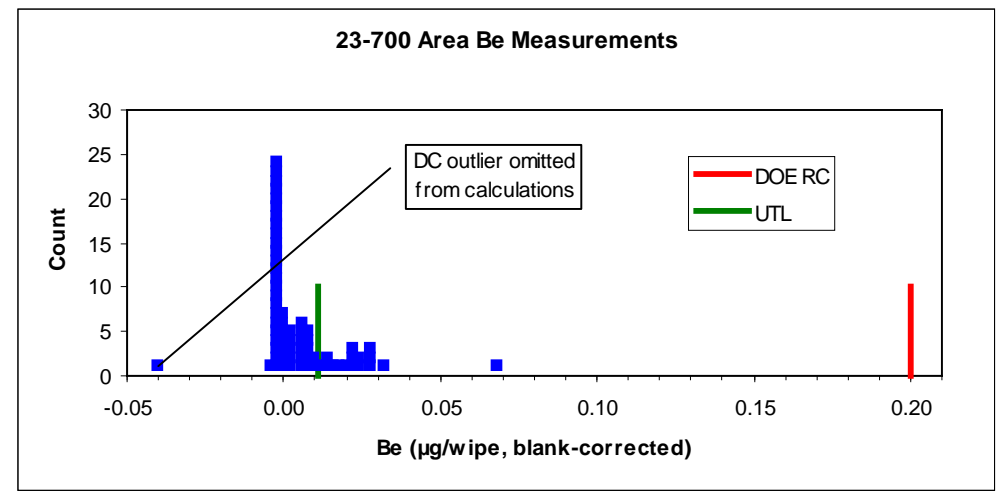

The extreme negative outlier in the shop Area was omitted from the UTL calculation.

\section{3-701}

\begin{tabular}{|rc|}
\hline 23-701 & \\
IWAs & \\
Type & FO \\
$\mathrm{N}=$ & 36 \\
$\mathrm{n}=$ & 10 \\
BC(DC) $=$ & 0.0003 \\
delta $=$ & 0.0030 \\
In sd $=$ & 0.893 \\
PL K $=$ & 3.813 \\
PLall $=$ & $\mathbf{0 . 2 6 7}$ \\
UTL K $=$ & 2.911 \\
UTL $=$ & $\mathbf{0 . 1 1 8}$ \\
Alpha $=$ & 0.05 \\
ProbPlot $\mathrm{R}=$ & 0.959 \\
&
\end{tabular}

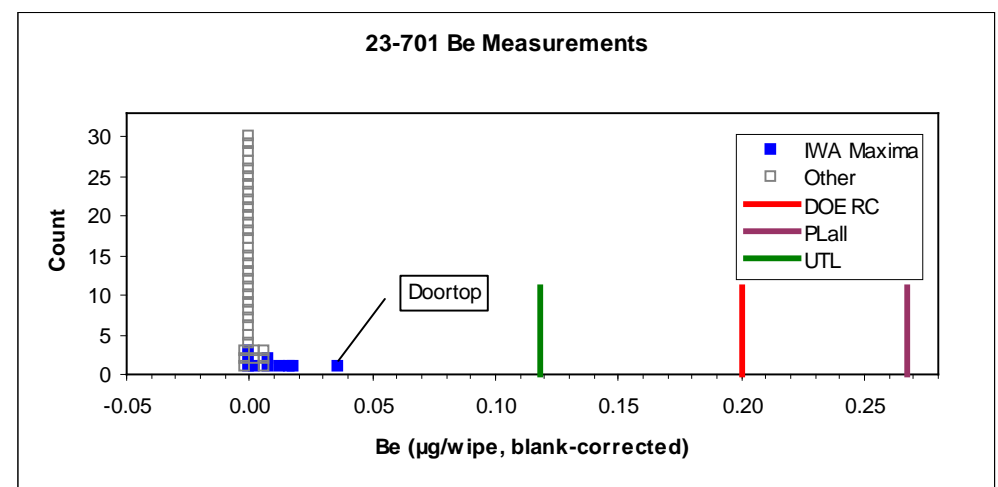




\begin{tabular}{|c|c|}
\hline \multicolumn{2}{|l|}{ 23-703 } \\
\hline Area & \\
\hline Type & ST \\
\hline $\mathrm{n}=$ & 59 \\
\hline $\mathrm{BC}(\mathrm{BNadj})=$ & -0.0062 \\
\hline BC(MS_new $)=$ & 0.0001 \\
\hline delta $=$ & 0.0085 \\
\hline mean of $\ln (x+$ delta $)=$ & -4.380 \\
\hline $\ln \mathrm{sd}=$ & 1.008 \\
\hline UTL K = & 2.026 \\
\hline UTL $=$ & 0.088 \\
\hline Alpha $=$ & 0.05 \\
\hline ProbPlot $\mathrm{R}=$ & 0.959 \\
\hline
\end{tabular}

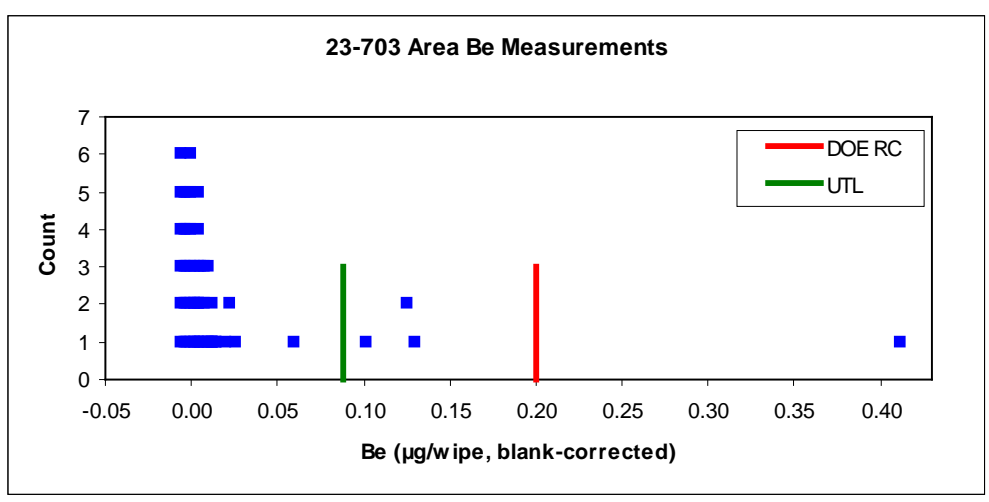

There is one wipe (MS_new) with Be greater than the DOE RC. The Be concentrations in three bulk samples obtained within the facility are low and their metal ratios are consistent with NTS soils. Be is somewhat elevated with respect to $\mathrm{Y}$ in the high wipe sample obtained on the floor, however.

\section{3-710}

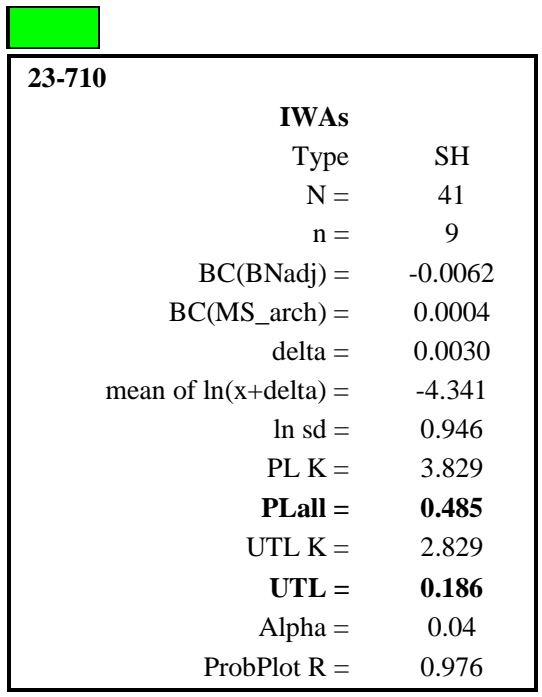

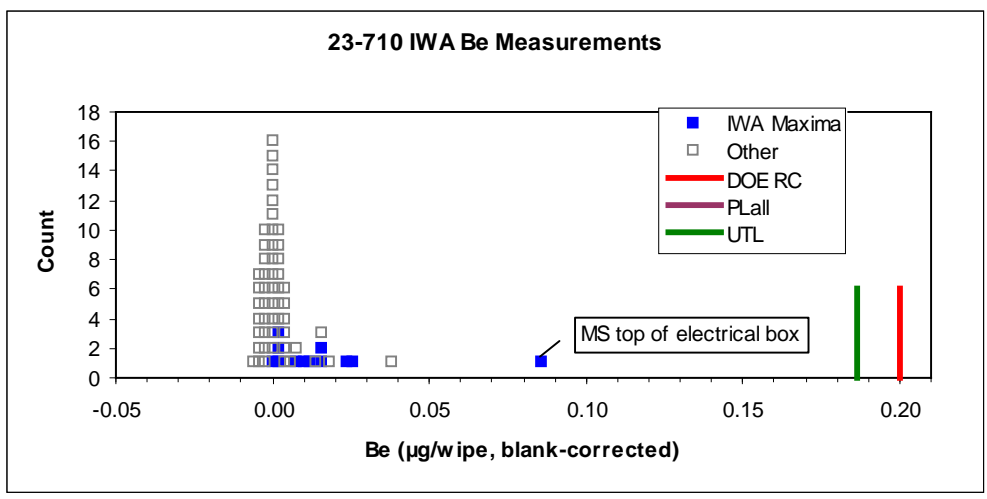

\begin{tabular}{|rc|}
\hline 23-710 & \\
Area & \\
Type & SH \\
$\mathrm{n}=$ & 104 \\
BC(BNadj $)=$ & -0.0062 \\
BC(MS_arch $)=$ & 0.0004 \\
delta $=$ & 0.0080 \\
mean of $\ln (\mathrm{x}+$ delta $)=$ & -4.562 \\
$\ln$ sd $=$ & 0.549 \\
UTL K $=$ & 2.047 \\
UTL $=$ & $\mathbf{0 . 0 2 4}$ \\
Alpha $=$ & 0.01 \\
ProbPlot R $=$ & 0.995 \\
&
\end{tabular}

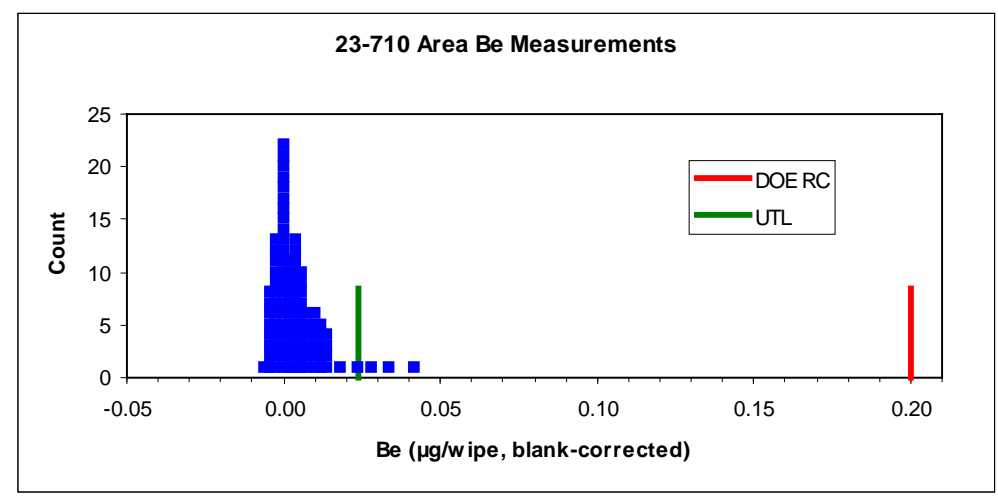




\begin{tabular}{|c|c|}
\hline \multicolumn{2}{|l|}{$23-725$} \\
\hline IWAs & \\
\hline Type & $\mathrm{CO}$ \\
\hline $\mathrm{N}=$ & 53 \\
\hline $\mathrm{n}=$ & 15 \\
\hline $\mathrm{BC}(\mathrm{DC})=$ & 0.0003 \\
\hline delta $=$ & 0.0020 \\
\hline mean of $\ln (x+$ delta $)=$ & -5.476 \\
\hline $\ln \mathrm{sd}=$ & 0.412 \\
\hline PL K = & 3.637 \\
\hline PLall = & 0.017 \\
\hline UTL $\mathrm{K}=$ & 2.566 \\
\hline $\mathbf{U T L}=$ & 0.010 \\
\hline Alpha $=$ & 0.05 \\
\hline ProbPlot $\mathrm{R}=$ & 0.862 \\
\hline
\end{tabular}

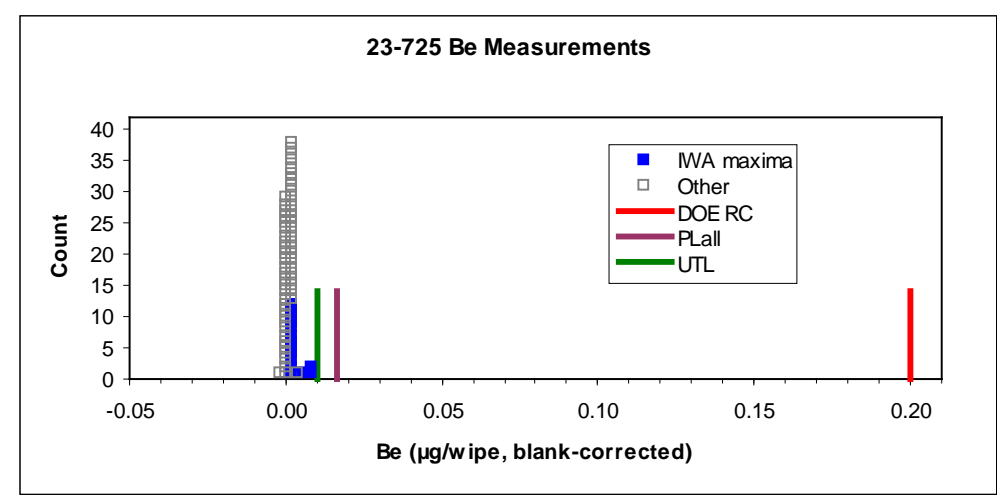

23-726

\begin{tabular}{|c|c|c|}
\hline \\
\hline \multicolumn{3}{|l|}{$23-726$} \\
\hline \multicolumn{3}{|c|}{ IWA } \\
\hline & Type & $\mathrm{CO}$ \\
\hline & $\mathrm{N}=$ & 72 \\
\hline & $\mathrm{n}=$ & 22 \\
\hline & $\mathrm{BC}(\mathrm{DC})=$ & 0.0003 \\
\hline & delta $=$ & 0.0020 \\
\hline & mean of $\ln (x+$ delta $)=$ & -5.064 \\
\hline & $\ln s d=$ & 1.320 \\
\hline & $\mathrm{PL} \mathrm{K}=$ & 3.530 \\
\hline & PLall = & 0.665 \\
\hline & UTL K = & 2.349 \\
\hline & UTL = & 0.138 \\
\hline & Alpha $=$ & 0.05 \\
\hline & ProbPlot $\mathrm{R}=$ & 0.861 \\
\hline \multicolumn{3}{|c|}{ omitting RF-screened room door top } \\
\hline & mean of $\ln (x+$ delta $)=$ & -5.305 \\
\hline & $\ln s d=$ & 0.729 \\
\hline & PLall $=$ & 0.063 \\
\hline & UTL $=$ & 0.026 \\
\hline & Alpha $=$ & 0.05 \\
\hline & ProbPlot $\mathrm{R}=$ & 0.988 \\
\hline
\end{tabular}

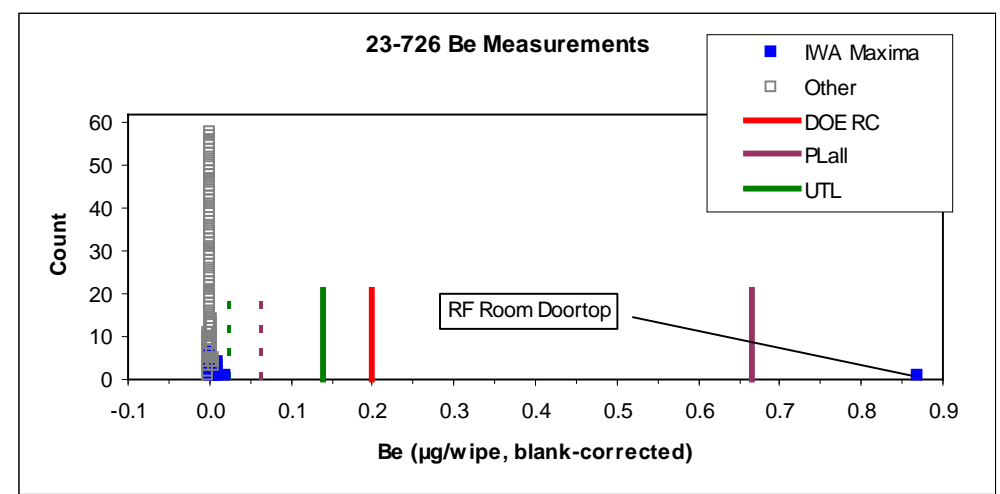

There is one anomalous value, obtained from a door top in the RF-screened room. This Be likely comes from a Be-Cu alloy used in the screening and, hence probably cannot be cleaned to the DOE RC reliably. It is suggested that the specific location be posted and potentially affected parties notified. Even with that value included, the UTL is below the DOE RC. With that value omitted, both the UTL and PLall are less than the RC. 


\begin{tabular}{|rc|}
\hline 23-750 & \\
IWAs & \\
Type & SH \\
$\mathrm{N}=$ & 16 \\
$\mathrm{n}=$ & 6 \\
BC(DC) $=$ & 0.0003 \\
delta $=$ & 0.0030 \\
mean of $\ln (\mathrm{x}+$ delta $)=$ & -5.451 \\
$\ln \mathrm{sd}=$ & 0.472 \\
$\mathrm{PL} \mathrm{K}=$ & 4.217 \\
PLall $=$ & $\mathbf{0 . 0 2 8}$ \\
UTL K $=$ & 3.918 \\
UTL $=$ & $\mathbf{0 . 0 2 4}$ \\
Alpha $=$ & 0.04 \\
ProbPlot $\mathrm{R}=$ & 0.979 \\
\hline
\end{tabular}

\begin{tabular}{|rc|}
\hline 23-750 & \\
Equipment bays etc & \\
Type & SH \\
$\mathrm{n}=$ & 48 \\
$\mathrm{BC}(\mathrm{DC})=$ & 0.0003 \\
delta $=$ & 0.0055 \\
mean of $\ln (\mathrm{x}+$ delta $)=$ & -4.988 \\
$\ln \mathrm{sd}=$ & 0.485 \\
$\mathrm{UTL} \mathrm{K}=$ & 2.285 \\
$\mathrm{UTL}=$ & $\mathbf{0 . 0 1 5}$ \\
Alpha $=$ & 0.01 \\
ProbPlot $\mathrm{R}=$ & 0.926 \\
\hline
\end{tabular}

\section{3-775 \& $776 \& 777$}

\begin{tabular}{|c|c|}
\hline \multicolumn{2}{|l|}{ 23-775 \& 776 \& 777} \\
\hline IWAs & \\
\hline Type & ST \\
\hline $\mathrm{N}=$ & 11 \\
\hline $\mathrm{n}=$ & 4 \\
\hline $\mathrm{BC}(\mathrm{DC})=$ & 0.0003 \\
\hline delta $=$ & 0.0030 \\
\hline mean of $\ln (x+$ delta $)=$ & -5.088 \\
\hline $\ln \mathrm{sd}=$ & 0.473 \\
\hline PL K = & 5.301 \\
\hline PLall $=$ & 0.073 \\
\hline UTL K = & 5.580 \\
\hline UTL = & 0.083 \\
\hline Alpha $=$ & 0.04 \\
\hline ProbPlot $\mathrm{R}=$ & 0.928 \\
\hline
\end{tabular}
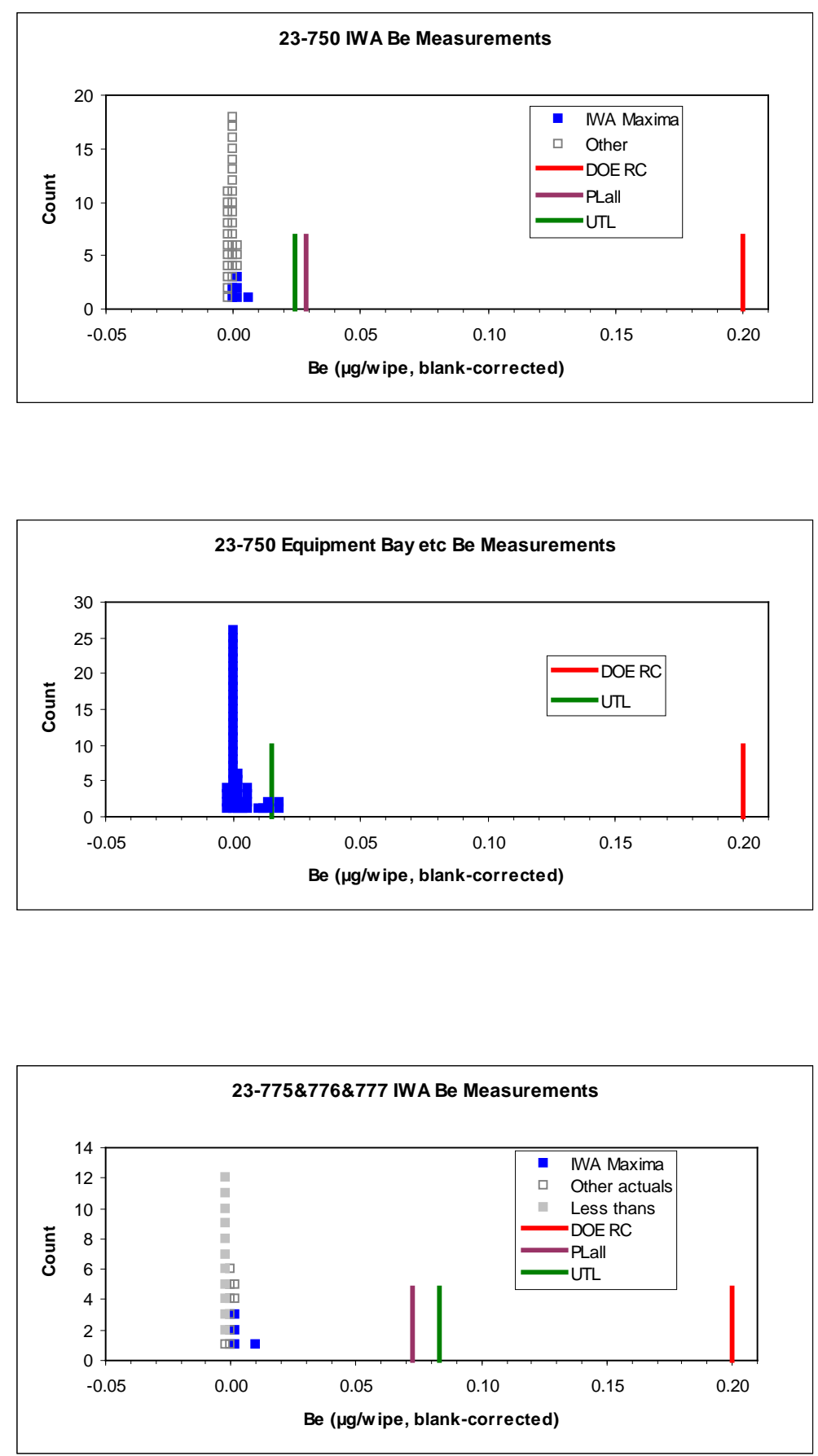

Some of the negative values in these early DC data were recorded only as " $<0$ "; hence censored-data UTL procedures were used. 


\begin{tabular}{|rc|}
\hline 23-775 \& 776 \& 777 & \\
Warehouse areas & \\
$\mathrm{n}=$ & 63 \\
shift $=$ & 0.0003 \\
delta $=$ & 0.0055 \\
ln sd $=$ & 0.587 \\
UTL K $=$ & 2.261 \\
UTL $=$ & $\mathbf{0 . 0 2 8}$ \\
Alpha $=$ & 0.01 \\
ProbPlot $\mathrm{R}=$ & 0.946
\end{tabular}

\section{3-B}

\begin{tabular}{|c|c|c|}
\hline \multirow[t]{15}{*}{ 23-B } & & \\
\hline & IWAs & \\
\hline & Type & OF \\
\hline & $\mathrm{N}=$ & 23 \\
\hline & $\mathrm{n}=$ & 8 \\
\hline & $\mathrm{BC}(\mathrm{DC})=$ & 0.0003 \\
\hline & delta $=$ & 0.0050 \\
\hline & mean of $\ln (x+$ delta $)=$ & -5.110 \\
\hline & $\ln \mathrm{sd}=$ & 0.254 \\
\hline & PL K = & 3.771 \\
\hline & PLall $=$ & 0.011 \\
\hline & UTL K = & 3.187 \\
\hline & UTL $=$ & 0.009 \\
\hline & Alpha = & 0.05 \\
\hline & ProbPlot $\mathrm{R}=$ & 0.968 \\
\hline
\end{tabular}
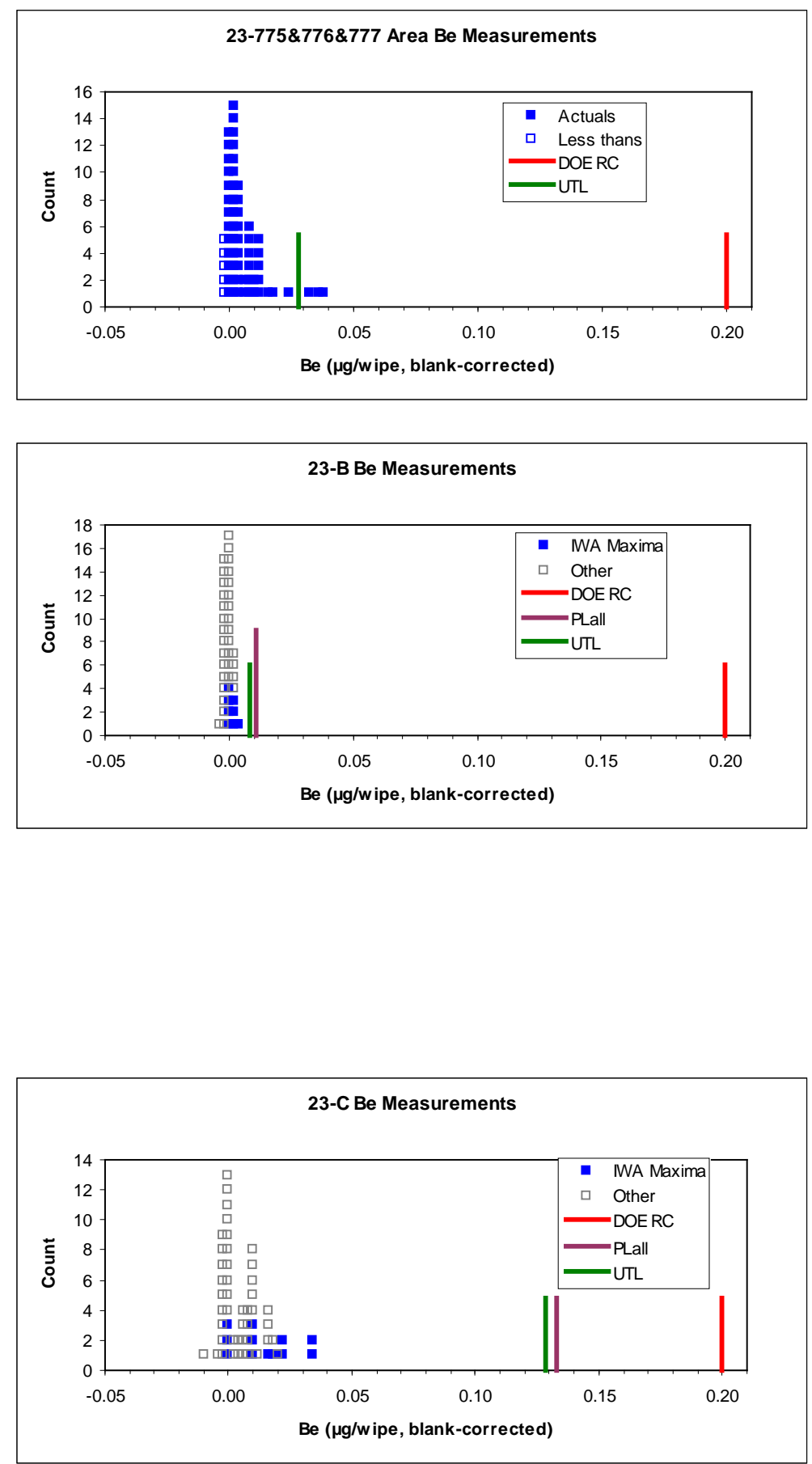

This facility did not pass using the PLall test with only $\mathrm{n}=8$ IWAs sampled, although none of the individual measurements exceeded the DOE RC. Four additional IWAs were subsequently sampled. 
23-D

\begin{tabular}{|c|c|c|}
\hline \multirow{2}{*}{\multicolumn{3}{|c|}{ 23-D }} \\
\hline & & \\
\hline & $\mathrm{N}=$ & 23 \\
\hline & $\mathrm{n}=$ & 8 \\
\hline & $\mathrm{BC}(\mathrm{BNadj})=$ & -0.0062 \\
\hline & delta $=$ & 0.0050 \\
\hline & mean of $\ln (x+$ delta $)=$ & -5.068 \\
\hline & $\ln \mathrm{sd}=$ & 0.166 \\
\hline & $\mathrm{PL} \mathrm{K}=$ & 3.771 \\
\hline & PLall = & 0.007 \\
\hline & UTL K = & 3.187 \\
\hline & UTL = & 0.006 \\
\hline & Alpha $=$ & 0.05 \\
\hline & ProbPlot $\mathrm{R}=$ & 0.955 \\
\hline
\end{tabular}

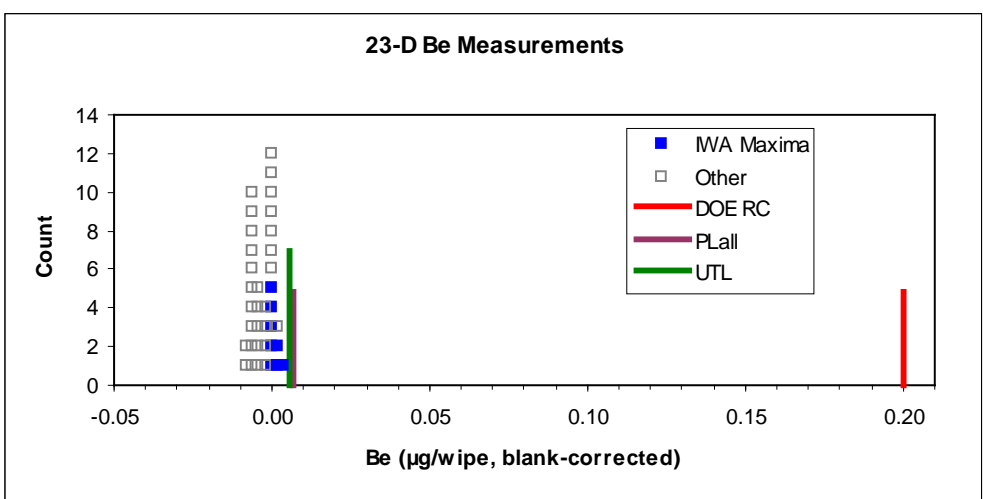

\section{3-W1}

\begin{tabular}{|c|c|}
\hline \multirow{2}{*}{\multicolumn{2}{|c|}{ 23-W1 }} \\
\hline & \\
\hline Туре & SH \\
\hline $\mathrm{N}=$ & 6 \\
\hline $\mathrm{n}=$ & 4 \\
\hline $\mathrm{BC}(\mathrm{DC})=$ & 0.0003 \\
\hline delta $=$ & 0.0030 \\
\hline mean of $\ln (x+$ delta $)=$ & -4.444 \\
\hline $\ln \mathrm{sd}=$ & 0.567 \\
\hline PL K = & 3.730 \\
\hline PLall $=$ & 0.094 \\
\hline UTL K = & 5.580 \\
\hline UTL = & 0.274 \\
\hline Alpha $=$ & 0.04 \\
\hline ProbPlot $\mathrm{R}=$ & 0.967 \\
\hline
\end{tabular}

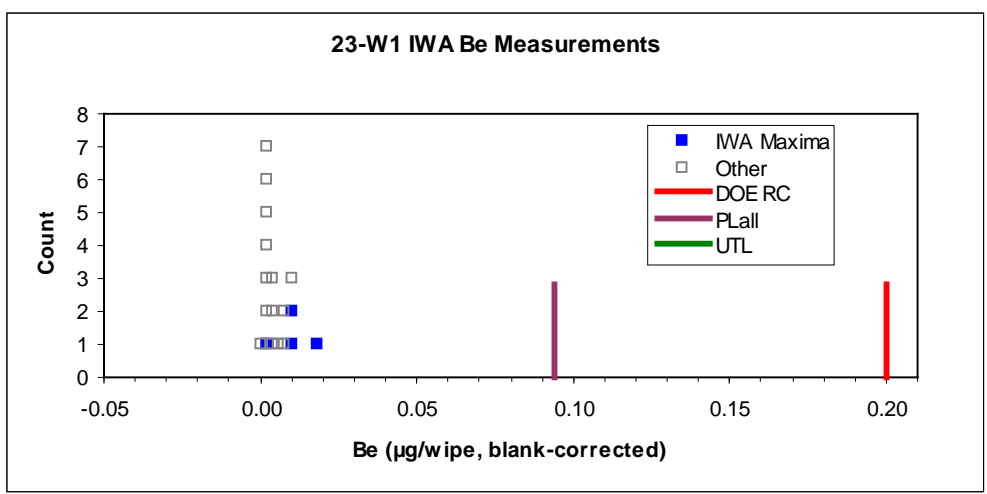

\begin{tabular}{|c|c|}
\hline 23-W1 & \\
\hline Shop areas & \\
\hline Туре & SH \\
\hline $\mathrm{n}=$ & 31 \\
\hline $\mathrm{BC}(\mathrm{DC})=$ & 0.0003 \\
\hline delta $=$ & 0.0055 \\
\hline mean of $\ln (\mathrm{x}+$ delta $)=$ & -4.588 \\
\hline $\ln \mathrm{sd}=$ & 0.704 \\
\hline UTL K = & 2.496 \\
\hline $\mathbf{U T L}=$ & 0.053 \\
\hline Alpha $=$ & 0.01 \\
\hline ProbPlot $\mathrm{R}=$ & 0.934 \\
\hline
\end{tabular}

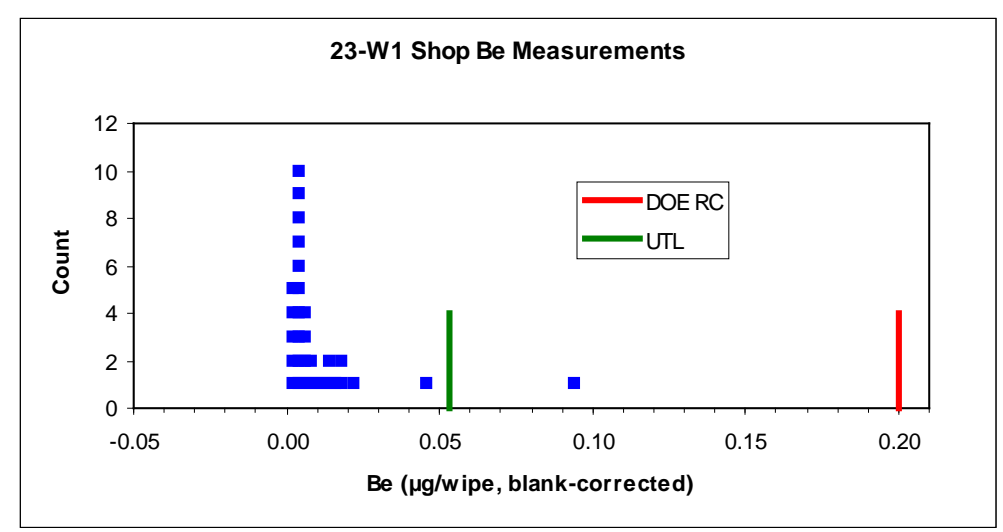


23-W2

\begin{tabular}{|c|c|}
\hline \multirow{2}{*}{\multicolumn{2}{|c|}{ 23-W2 }} \\
\hline & \\
\hline Tyре & SH \\
\hline $\mathrm{N}=$ & 10 \\
\hline $\mathrm{n}=$ & 7 \\
\hline $\mathrm{BC}(\mathrm{DC})=$ & 0.0003 \\
\hline delta $=$ & 0.0030 \\
\hline mean of $\ln (\mathrm{x}+$ delta $)=$ & -4.317 \\
\hline $\ln \mathrm{sd}=$ & 0.542 \\
\hline PL K = & 3.042 \\
\hline PLall $=$ & 0.066 \\
\hline UTL K = & 3.569 \\
\hline UTL = & 0.089 \\
\hline Alpha $=$ & 0.04 \\
\hline ProbPlot $\mathrm{R}=$ & 0.976 \\
\hline
\end{tabular}

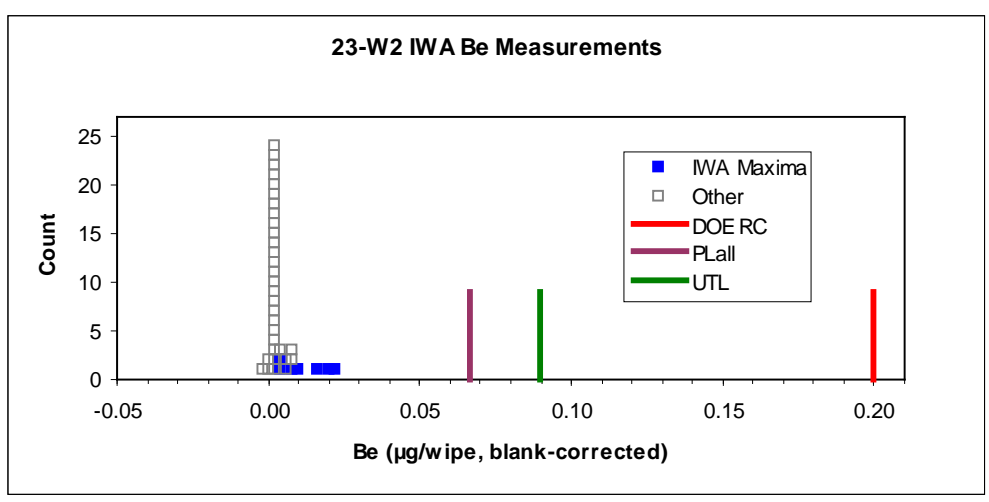

\begin{tabular}{|rc|}
\hline 23-W2 & \\
Shop areas & \\
Type & SH \\
$\mathrm{n}=$ & 24 \\
BC(DC) $=$ & 0.0003 \\
delta $=$ & 0.0055 \\
mean of $\ln (\mathrm{x}+$ delta $)=$ & -4.664 \\
$\ln \mathrm{sd}=$ & 0.565 \\
UTL $\mathrm{K}=$ & 2.662 \\
UTL $=$ & $\mathbf{0 . 0 3 7}$ \\
Alpha $=$ & 0.01 \\
ProbPlot $\mathrm{R}=$ & 0.808 \\
\hline
\end{tabular}

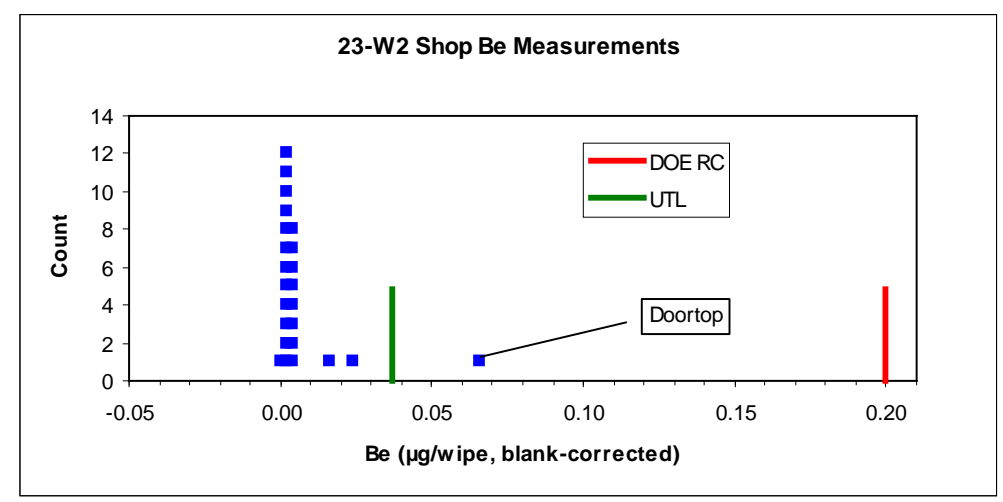

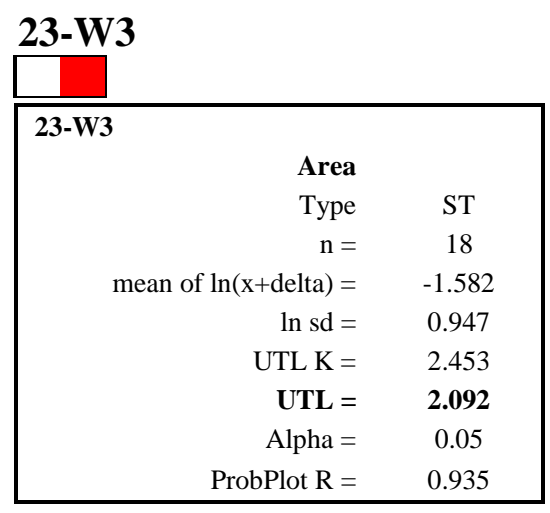

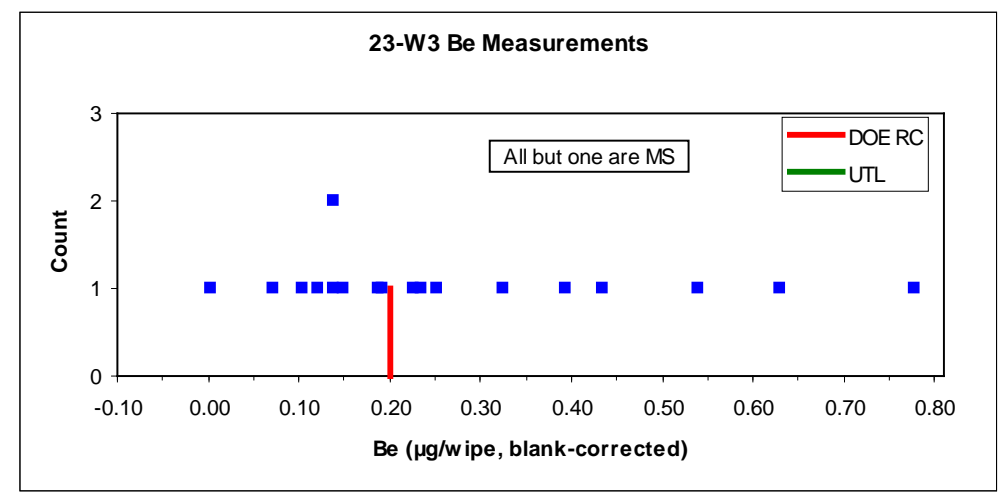

Nine of eighteen MS_arch data are above the DOE RC. This facility has been demolished. 


\section{3-W5}

The one IWA was sampled; the maximum value was $0.030 \mu \mathrm{g} /$ wipe.

\begin{tabular}{|rc|}
\hline 23-W5 & \\
Area & \\
Type & ST \\
$\mathrm{n}=$ & 26 \\
BC(BNadj $)=$ & -0.0062 \\
BC(MS_arch $)=$ & 0.0004 \\
delta $=$ & 0.0080 \\
mean of $\ln (\mathrm{x}+$ delta $)=$ & -4.459 \\
$\ln$ sd $=$ & 0.558 \\
UTL $\mathrm{K}=$ & 2.275 \\
UTL $=$ & $\mathbf{0 . 0 3 3}$ \\
Alpha $=$ & 0.05 \\
ProbPlot $\mathrm{R}=$ & 0.964 \\
\hline
\end{tabular}

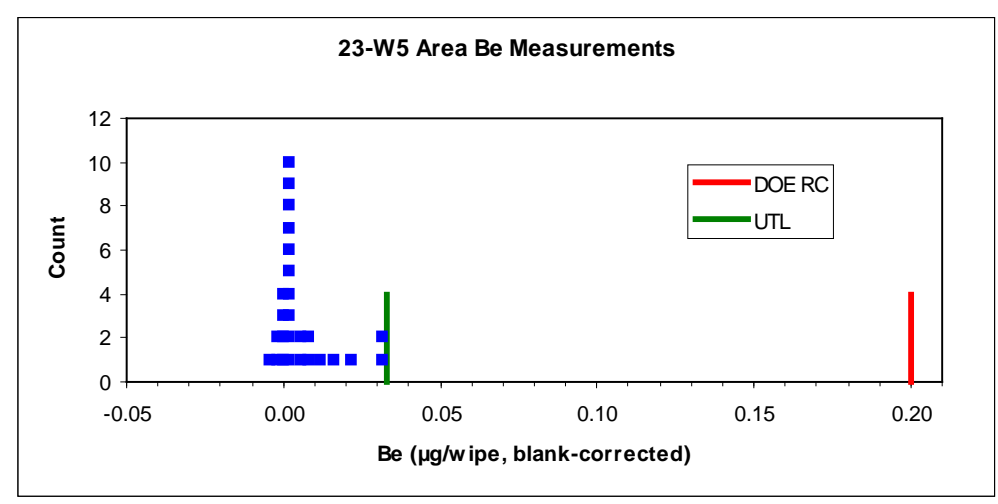

\section{3-W6}

\begin{tabular}{|c|c|}
\hline \multirow{2}{*}{\multicolumn{2}{|c|}{ 23-W6 }} \\
\hline & \\
\hline Type & $\mathrm{SH}$ \\
\hline $\mathrm{N}=$ & 12 \\
\hline $\mathrm{n}=$ & 8 \\
\hline $\mathrm{BC}(\mathrm{BNadj})=$ & -0.0062 \\
\hline BC(MS_arch $)=$ & 0.0004 \\
\hline delta $=$ & 0.0030 \\
\hline mean of $\ln (x+$ delta $)=$ & -3.343 \\
\hline $\ln \mathrm{sd}=$ & 0.542 \\
\hline $\mathrm{PL} \mathrm{K}=$ & 2.926 \\
\hline PLall $=$ & 0.170 \\
\hline UTL K = & 3.187 \\
\hline UTL = & 0.196 \\
\hline Alpha $=$ & 0.05 \\
\hline ProbPlot $\mathrm{R}=$ & 0.980 \\
\hline
\end{tabular}

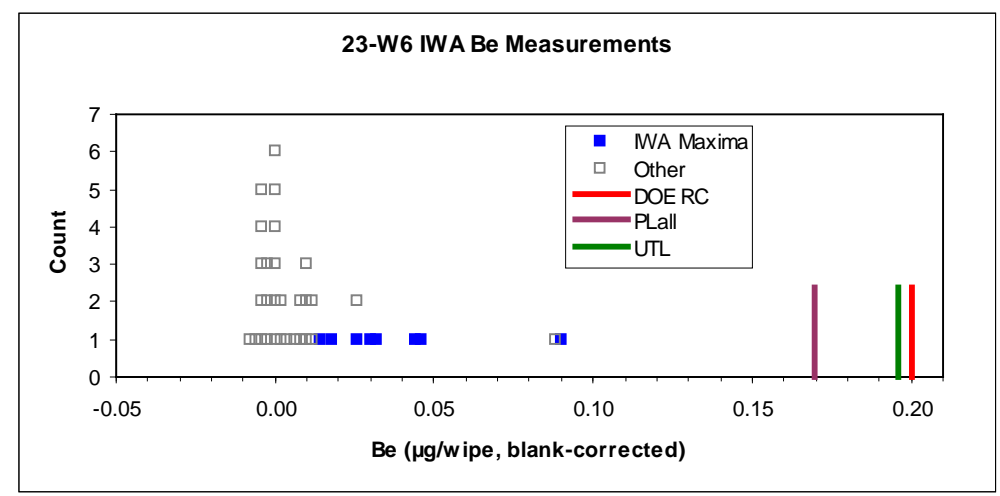




\begin{tabular}{|c|c|c|}
\hline \multicolumn{3}{|c|}{ 24-A01 } \\
\hline \multicolumn{3}{|c|}{ 24-A01 } \\
\hline & IWAs & \\
\hline & Type & $\mathrm{OF}$ \\
\hline \multicolumn{3}{|c|}{ Conservative } \\
\hline & $\mathrm{N}=$ & 140 \\
\hline & $\mathrm{n}=$ & 59 \\
\hline & mean of $\ln (x+$ delta $)=$ & -5.305 \\
\hline & $\ln \mathrm{sd}=$ & 0.872 \\
\hline & PL K = & 3.526 \\
\hline & PLall $=$ & 0.107 \\
\hline & UTL K = & 2.100 \\
\hline & UTL $=$ & 0.003 \\
\hline & NPUTL $=$ & 0.072 \\
\hline & Alpha $=$ & 0.05 \\
\hline \multicolumn{3}{|c|}{ Alternate } \\
\hline & mean of $\ln (x+$ delta $)=$ & -5.296 \\
\hline & $\ln s d=$ & 0.783 \\
\hline & PLall $=$ & 0.079 \\
\hline & $\mathbf{U T L}=$ & 0.003 \\
\hline & NPUTL $=$ & 0.072 \\
\hline
\end{tabular}

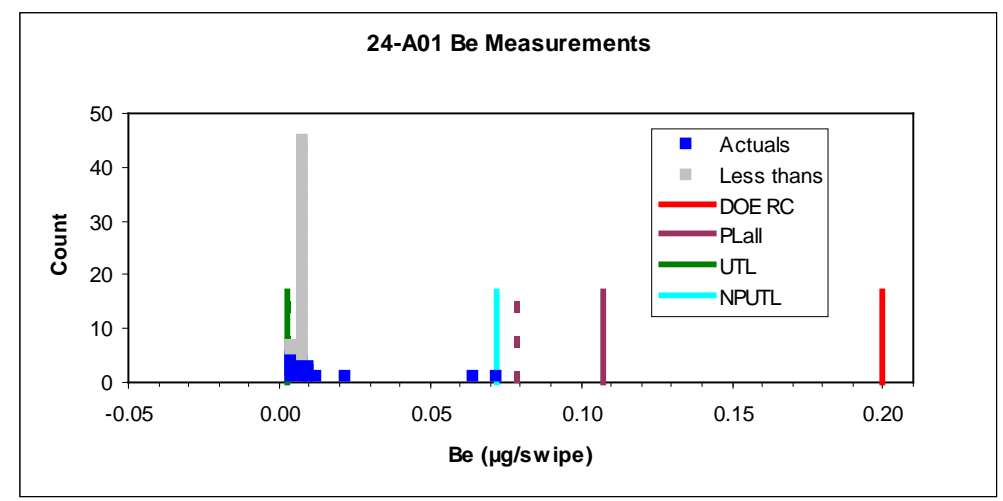

This facility had been abandoned before the Worker Environment Survey commenced. A sampling plan was developed following the principles set out in Appendix 1, and then old data were found to fit that plan as nearly as possible. These data came from variety of samplings and laboratories. Determining the maximum value for a given IWA was somewhat problematic because of differing reporting limits and the general unavailability of uncensored data. Two approaches were used. The conservative approach uses the highest value for any IWA, whether an actual value or a reporting limit. The alternate approach uses the highest actual value found, if any, and otherwise the highest reporting limit. Censored data maximum likelihood estimates are used as appropriate; this approach allows a variety of different reporting limits (see "Parametric 95\%-95\% Upper Tolerance Limits for Censored Lognormal Data,” C. B. Davis, presented at the Joint Statistical Meetings, Seattle, WA, 2006).

The NPUTL (nonparametric upper tolerance limit) is the largest of 59 observations. It is considerably above the UTL value, reflecting the fact that the two highest values are outliers. Only the IWA maxima are shown; the solid squares are actual values and the empty square are "less than" values, plotted at half their respective reporting limits. The UTL values are buried in the data in either case. 


\section{4-A02}

Room 5082, the Readiness Warehouse located at the east end, is not accessible from the rest of the building and is only incidentally occupied. It is treated as a separate facility.

\begin{tabular}{|rc|}
\hline 24-A02 & \\
IWAs & \\
Type & OF \\
$\mathrm{N}=$ & 95 \\
$\mathrm{n}=$ & 27 \\
BC(DC) $=$ & 0.0003 \\
delta $=$ & 0.005 \\
mean of $\ln (\mathrm{x}+$ delta $)=$ & -5.136 \\
$\ln$ sd $=$ & 0.259 \\
PL $\mathrm{K}=$ & 3.654 \\
PLall $=$ & $\mathbf{0 . 0 1 0}$ \\
UTL K $=$ & 2.307 \\
UTL $=$ & $\mathbf{0 . 0 0 6}$ \\
Alpha $=$ & 0.04 \\
ProbPlot $\mathrm{R}=$ & 0.974 \\
\end{tabular}

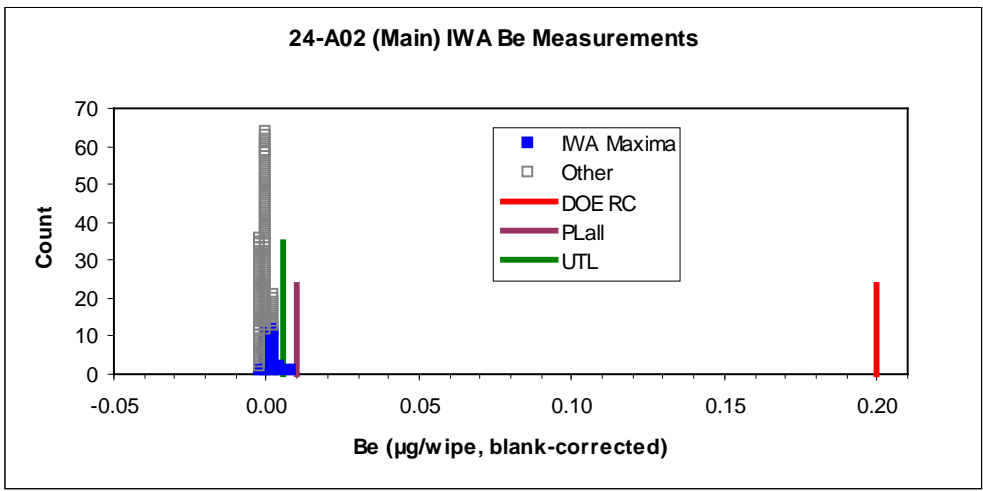

\begin{tabular}{|rc|}
\hline 24-A02 & \\
5001 Area & \\
Type & ST \\
$\mathrm{n}=$ & 15 \\
BC(DC) $=$ & 0.0003 \\
delta $=$ & 0.0055 \\
mean of $\ln (\mathrm{x}+$ delta $)=$ & -5.416 \\
$\ln \mathrm{sd}=$ & 0.279 \\
UTL $\mathrm{K}=$ & 3.102 \\
UTL $=$ & $\mathbf{0 . 0 0 5}$ \\
Alpha $=$ & 0.01 \\
ProbPlot $\mathrm{R}=$ & 0.956 \\
\hline
\end{tabular}

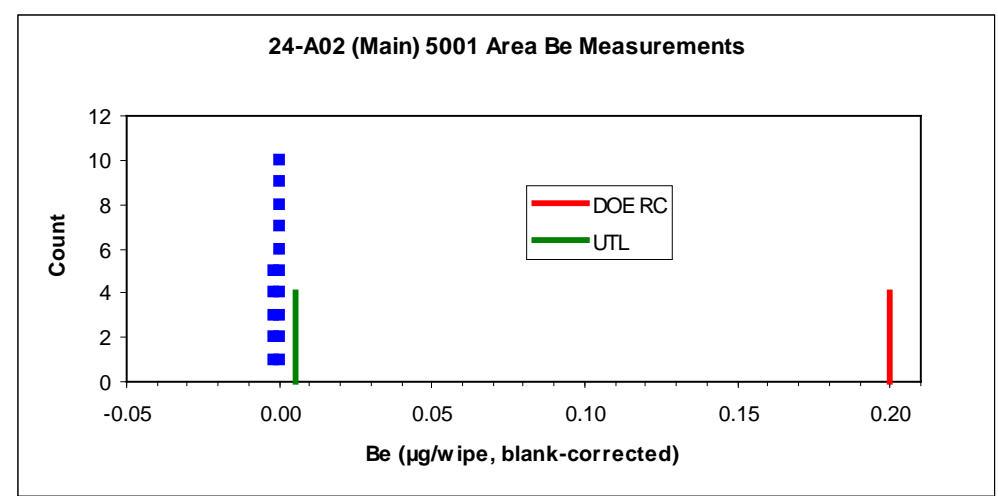

24-A02 Readiness Warehouse (Room 5082)

\begin{tabular}{|c|c|c|}
\hline \multicolumn{3}{|c|}{ 24-A02 } \\
\hline & 5082 & \\
\hline & Type & ST \\
\hline & $\mathrm{n}=$ & 62 \\
\hline & $\mathrm{BC}(\mathrm{DC})=$ & 0.0003 \\
\hline & NPUTL = & 0.200 \\
\hline & Alpha = & 0.042 \\
\hline
\end{tabular}

The nonparametric UTL (NPUTL)

is the largest observation with a total of 59 to 92 observations. 


\section{4-A04}

\begin{tabular}{|c|c|}
\hline \multirow{2}{*}{\multicolumn{2}{|c|}{ 24-A04 }} \\
\hline & \\
\hline Туре & SH \\
\hline $\mathrm{N}=$ & 57 \\
\hline $\mathrm{n}=$ & 24 \\
\hline $\mathrm{BC}(\mathrm{BNadj})=$ & -0.0062 \\
\hline BC(DC,Ghost $)=$ & 0.0013 \\
\hline BC $($ DC, Whatmann $)=$ & 0.0003 \\
\hline BC(MS_arch) = & 0.0004 \\
\hline delta $=$ & 0.0030 \\
\hline mean of $\ln (x+$ delta $)=$ & -4.238 \\
\hline $\ln \mathrm{sd}=$ & 1.166 \\
\hline PL K = & 3.426 \\
\hline PLall $=$ & 0.782 \\
\hline UTL K = & 2.360 \\
\hline UTL = & 0.223 \\
\hline Alpha $=$ & 0.04 \\
\hline ProbPlot $\mathrm{R}=$ & 0.977 \\
\hline \multicolumn{2}{|c|}{ omitting 2 door tops } \\
\hline mean of $\ln (x+$ delta $)=$ & -4.365 \\
\hline $\ln s d=$ & 0.991 \\
\hline PLall $=$ & 0.377 \\
\hline $\mathbf{U T L}=$ & 0.129 \\
\hline ProbPlot $\mathrm{R}=$ & 0.974 \\
\hline \multicolumn{2}{|c|}{ UTL cluster analysis } \\
\hline $\mathrm{F}=$ & 5.12 \\
\hline delta $=$ & 0.0090 \\
\hline mean of $\ln (x+$ delta $)=$ & -4.248 \\
\hline $\operatorname{tau}^{\wedge} 2=$ & 0.209 \\
\hline $\operatorname{sig} \wedge 2=$ & 0.203 \\
\hline $\ln s d=$ & 0.641 \\
\hline UTL K = & 2.086 \\
\hline UTL $=$ & 0.046 \\
\hline ProbPlot $\mathrm{R}=$ & 0.928 \\
\hline
\end{tabular}

\begin{tabular}{|c|c|}
\hline \multicolumn{2}{|l|}{ 24-A04 } \\
\hline Area & \\
\hline Type & $\mathrm{SH}$ \\
\hline $\mathrm{n}=$ & 36 \\
\hline $\mathrm{BC}(\mathrm{DC}$, Whatmann $)=$ & 0.0003 \\
\hline delta $=$ & 0.0055 \\
\hline mean of $\ln (x+$ delta $)=$ & -5.117 \\
\hline $\ln s d=$ & 0.396 \\
\hline UTL K = & 2.415 \\
\hline UTL $=$ & 0.010 \\
\hline Alpha $=$ & 0.01 \\
\hline ProbPlot $\mathrm{R}=$ & 0.914 \\
\hline
\end{tabular}

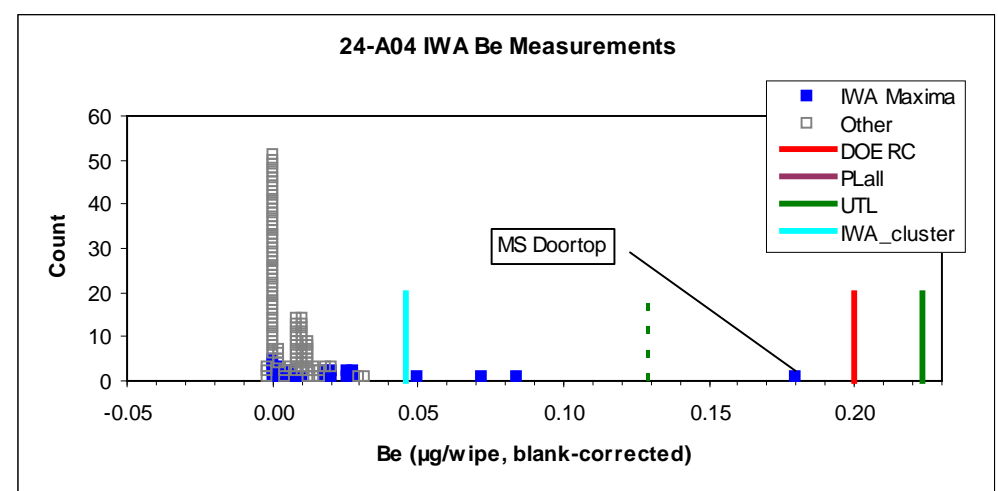

None of the data values exceed the DOE RC; there are a couple of high MS_arch values which push PLall and UTL up.

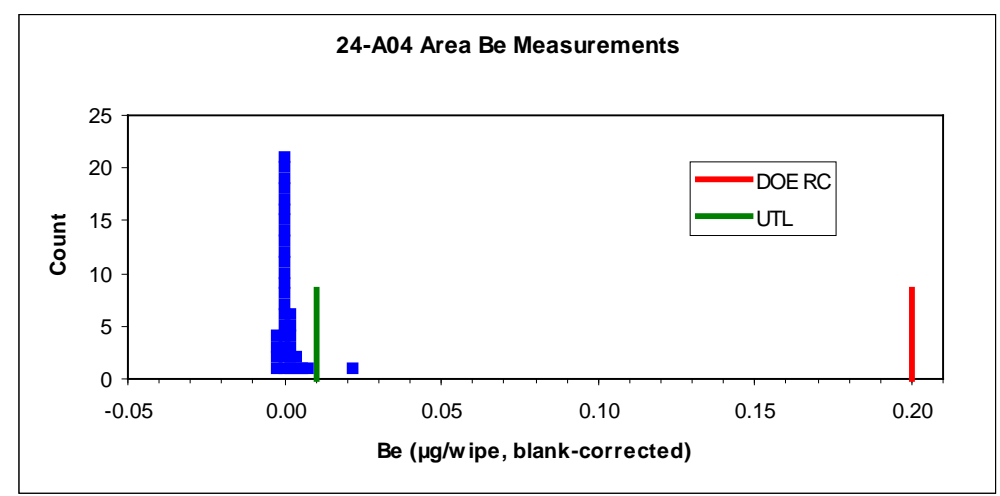




\section{4-A05}

\begin{tabular}{|c|c|}
\hline \multicolumn{2}{|l|}{ 24-A05 } \\
\hline IWAs & \\
\hline Type & ST \\
\hline $\mathrm{N}=$ & 5 \\
\hline $\mathrm{n}=$ & 4 \\
\hline $\mathrm{BC}(\mathrm{BNadj})=$ & -0.0062 \\
\hline $\mathrm{BC}(\mathrm{DC})=$ & 0.0003 \\
\hline BC(MS_arch) $=$ & 0.0004 \\
\hline delta $=$ & 0.0030 \\
\hline mean of $\ln (x+$ delta $)=$ & -2.445 \\
\hline $\ln \mathrm{sd}=$ & 1.266 \\
\hline $\mathrm{PL} \mathrm{K}=$ & 2.631 \\
\hline PLall = & 2.420 \\
\hline UTL K = & 5.144 \\
\hline UTL = & 58.284 \\
\hline Alpha $=$ & 0.05 \\
\hline ProbPlot $\mathrm{R}=$ & 0.865 \\
\hline \multicolumn{2}{|c|}{ omitting MS_arch on electrical box } \\
\hline mean of $\ln (x+$ delta $)=$ & -2.977 \\
\hline $\ln \mathrm{sd}=$ & 0.250 \\
\hline PLall $=$ & 0.095 \\
\hline UTL $=$ & 0.181 \\
\hline ProbPlot $\mathrm{R}=$ & 0.994 \\
\hline
\end{tabular}

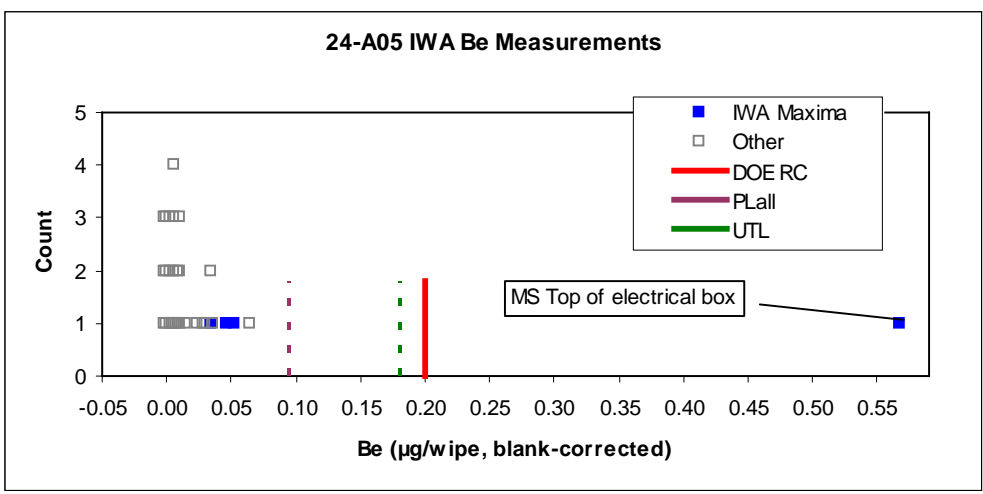

The high value (MS_arch) is in a location not typically touched in normal work practice. The Be concentrations in the other wipes are far below the DOE RC.

\section{4-A07}

\begin{tabular}{|c|c|}
\hline \multicolumn{2}{|l|}{ 24-A07 } \\
\hline IWAs & \\
\hline Type & FO \\
\hline $\mathrm{N}=$ & 3 \\
\hline $\mathrm{n}=$ & 2 \\
\hline $\mathrm{BC}(\mathrm{DC})=$ & 0.0003 \\
\hline delta $=$ & 0.0030 \\
\hline mean of $\ln (x+$ delta $)=$ & -4.733 \\
\hline $\ln s d=$ & 0.473 \\
\hline PL K = & 7.733 \\
\hline PLall $=$ & 0.342 \\
\hline Alpha $=$ & 0.05 \\
\hline \multicolumn{2}{|c|}{ UTL cluster sampling analysis } \\
\hline $\mathrm{F}=$ & 0.45 \\
\hline delta $=$ & 0.0055 \\
\hline mean of $\ln (x+$ delta $)=$ & -5.016 \\
\hline $\ln \mathrm{sd}=$ & 0.335 \\
\hline UTL K = & 2.911 \\
\hline UTL = & 0.018 \\
\hline ProbPlot $\mathrm{R}=$ & 0.886 \\
\hline
\end{tabular}

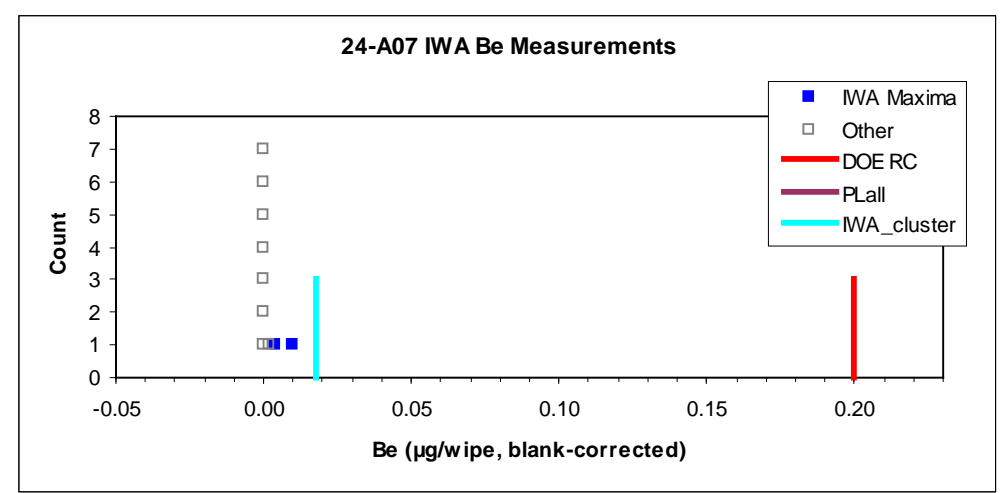

The PLall and UTL multipliers are quite high with very few IWAs sampled. 


\section{4-A12}

\begin{tabular}{|c|c|}
\hline \multicolumn{2}{|l|}{ 24-A12 } \\
\hline IWAs & \\
\hline Type & $\mathrm{SH}$ \\
\hline $\mathrm{N}=$ & 38 \\
\hline $\mathrm{n}=$ & 10 \\
\hline $\mathrm{BC}(\mathrm{DC})=$ & 0.0003 \\
\hline delta $=$ & 0.0030 \\
\hline mean of $\ln (x+$ delta $)=$ & -5.425 \\
\hline $\ln \mathrm{sd}=$ & 0.209 \\
\hline PL K = & 3.853 \\
\hline PLall = & 0.007 \\
\hline UTL K = & 2.911 \\
\hline UTL = & 0.005 \\
\hline Alpha $=$ & 0.05 \\
\hline ProbPlot $\mathrm{R}=$ & 0.995 \\
\hline
\end{tabular}

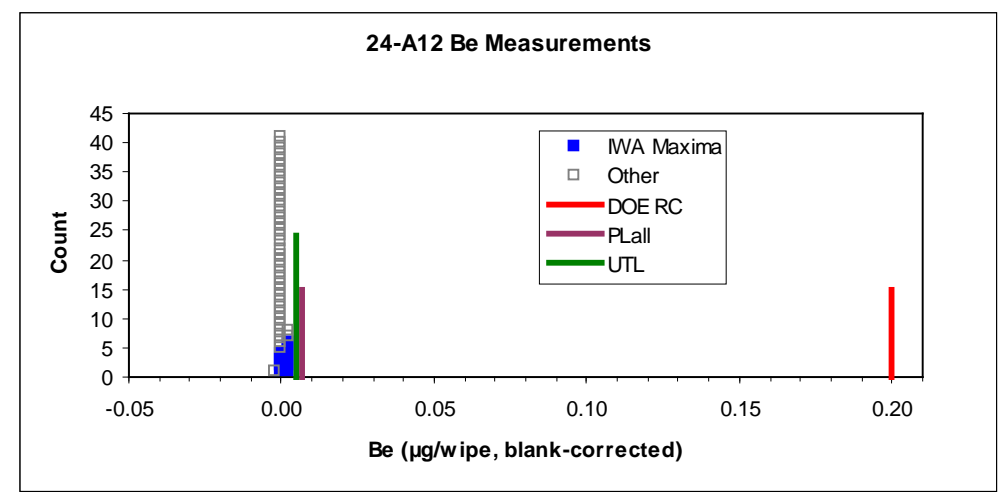

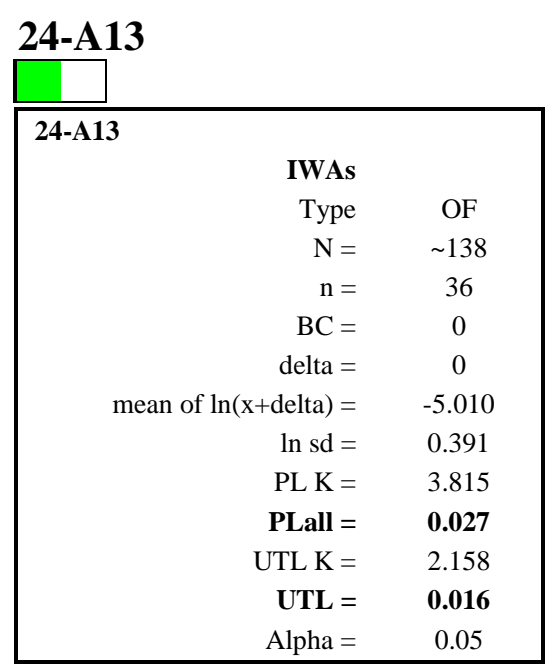

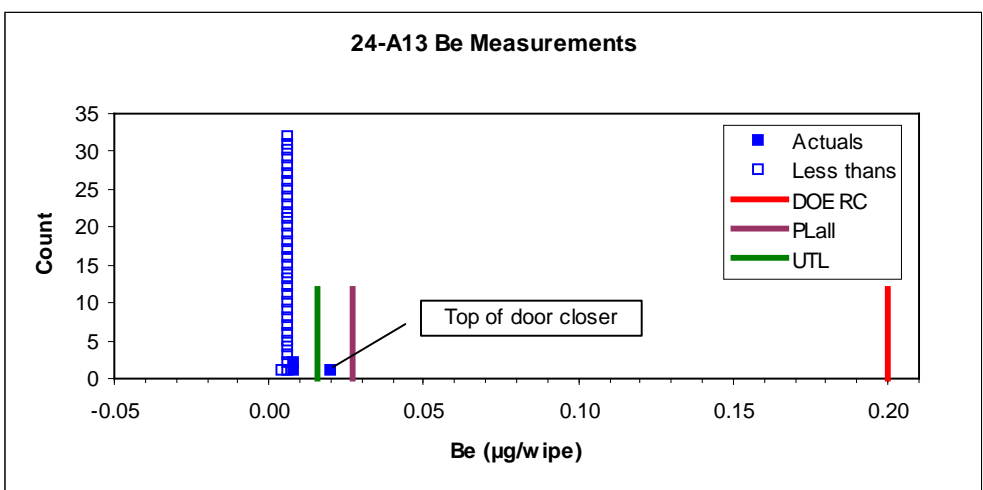

This facility was sampled prior to the organized Be sampling campaign. Available data are used to simulate a sampling plan that might have been used if the Appendix 1 principles had been followed. All data values are quite low; this substitution is quite reasonable.

\section{4-A15}

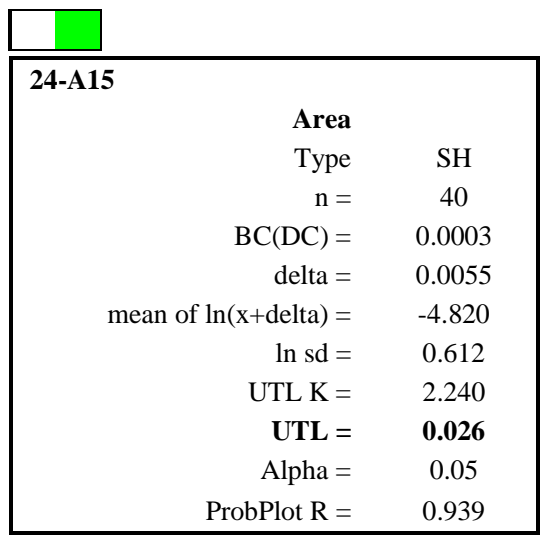

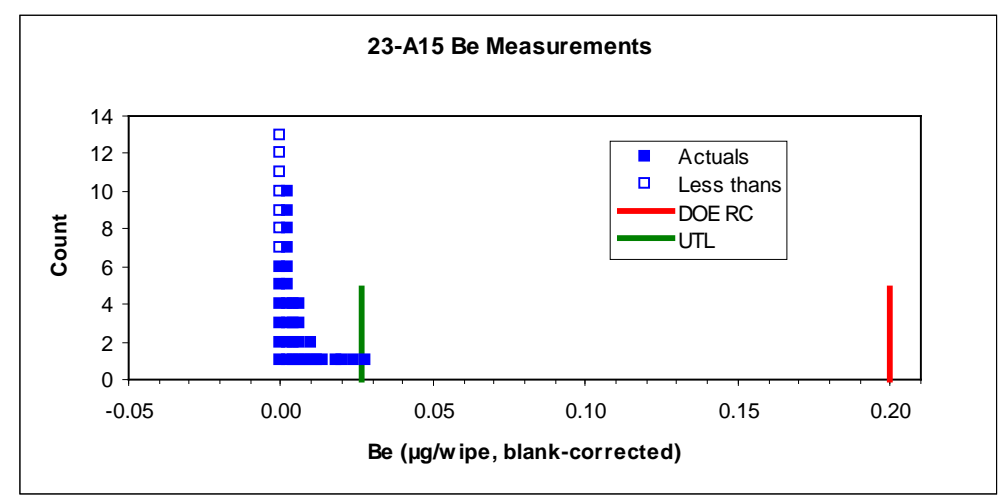




\section{4-B01}

\begin{tabular}{|c|c|}
\hline \multicolumn{2}{|l|}{ 24-B01 } \\
\hline IWAs & \\
\hline Type & OF \\
\hline$N=$ & 217 \\
\hline $\mathrm{n}=$ & 56 \\
\hline $\mathrm{BC}(\mathrm{DC}, \mathrm{G})=$ & 0.0041 \\
\hline $\mathrm{BC}(\mathrm{DC}, \mathrm{H})=$ & 0.0003 \\
\hline delta $=$ & 0.0050 \\
\hline mean of $\ln (\mathrm{x}+$ delta $)=$ & -5.110 \\
\hline $\ln s d=$ & 0.611 \\
\hline PL K = & 3.632 \\
\hline PLall $=$ & 0.051 \\
\hline UTL K = & 2.038 \\
\hline UTL $=$ & 0.016 \\
\hline Alpha $=$ & 0.05 \\
\hline ProbPlot $\mathrm{R}=$ & 0.979 \\
\hline
\end{tabular}

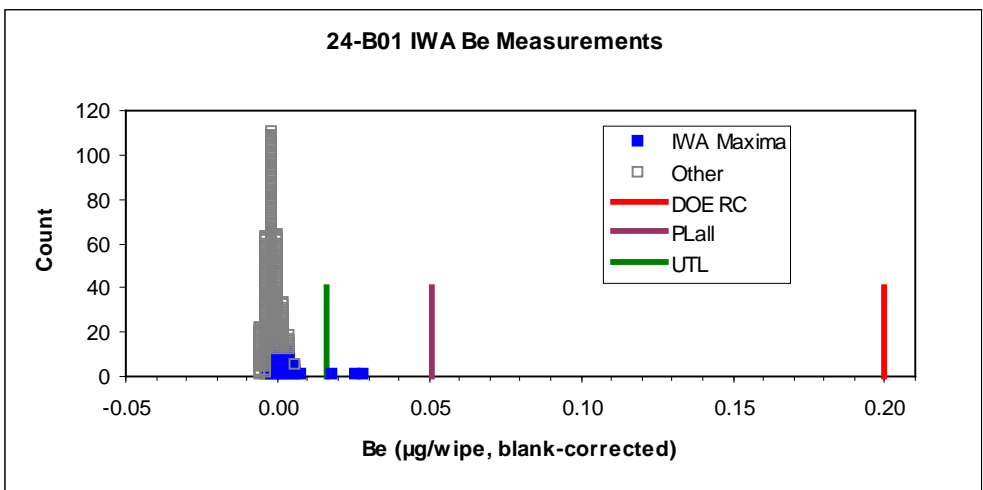

\section{4-B02}

\begin{tabular}{|c|c|}
\hline \multirow{2}{*}{\multicolumn{2}{|c|}{ 24-B02 }} \\
\hline & \\
\hline Туре & OF \\
\hline $\mathrm{N}=$ & 65 \\
\hline $\mathrm{n}=$ & 17 \\
\hline $\mathrm{BC}(\mathrm{DC}, \mathrm{G})=$ & 0.0041 \\
\hline delta $=$ & 0.005 \\
\hline mean of $\ln (x+$ delta $)=$ & -4.948 \\
\hline $\ln \mathrm{sd}=$ & 0.342 \\
\hline $\mathrm{PL} \mathrm{K}=$ & 3.656 \\
\hline PLall = & 0.020 \\
\hline UTL K = & 2.486 \\
\hline UTL = & 0.012 \\
\hline Alpha $=$ & 0.05 \\
\hline ProbPlot $\mathrm{R}=$ & 0.977 \\
\hline
\end{tabular}

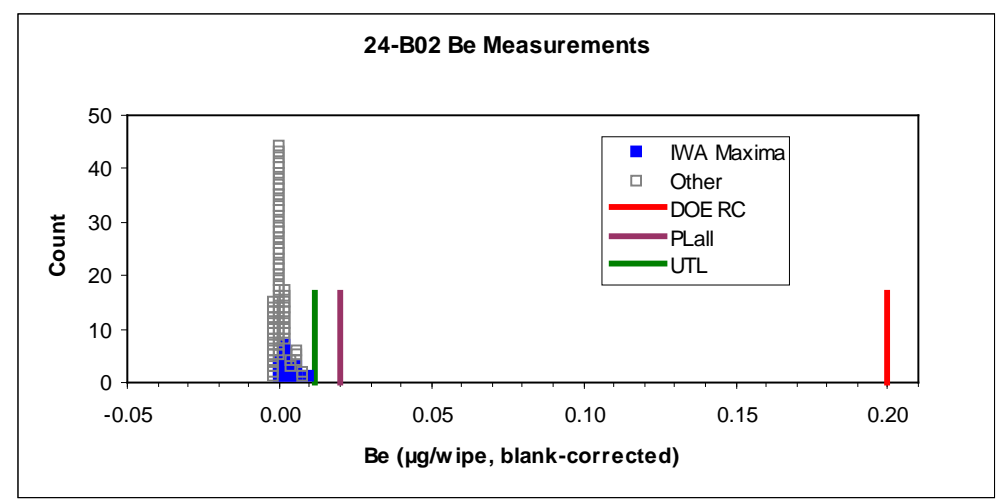




\section{4-B03}

\begin{tabular}{|c|c|}
\hline \multicolumn{2}{|l|}{ 24-B03 } \\
\hline IWAs & \\
\hline Type & OF \\
\hline $\mathrm{N}=$ & 385 \\
\hline $\mathrm{n}=$ & 66 \\
\hline $\mathrm{BC}(\mathrm{G})=$ & 0.0041 \\
\hline $\mathrm{BC}(\mathrm{H})=$ & 0.0003 \\
\hline delta $=$ & 0.005 \\
\hline mean of $\ln (x+$ delta $)=$ & -4.665 \\
\hline $\ln \mathrm{sd}=$ & 0.447 \\
\hline PLall $\mathrm{K}=$ & 3.756 \\
\hline PLall $=$ & 0.046 \\
\hline UTL K = & 1.964 \\
\hline UTL $=$ & 0.018 \\
\hline Alpha $=$ & 0.05 \\
\hline ProbPlot $\mathrm{R}=$ & 0.886 \\
\hline
\end{tabular}

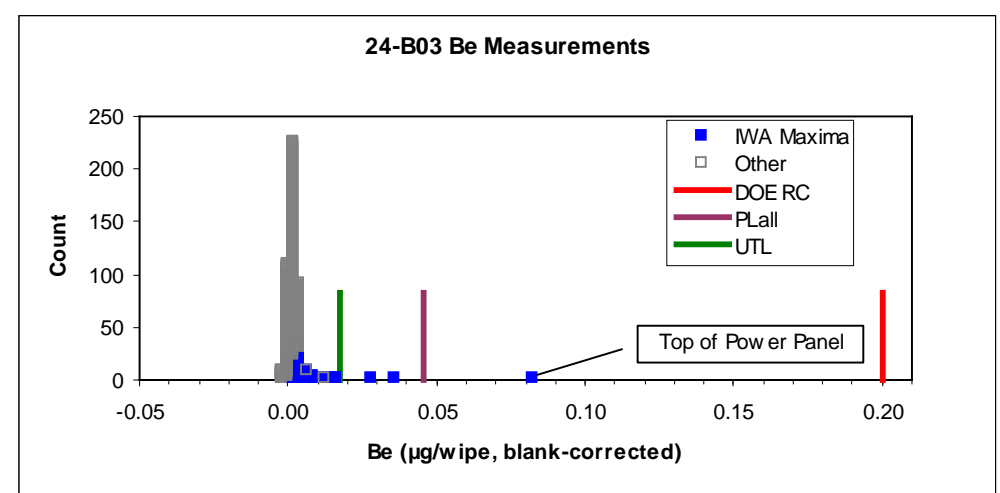

These samples from B01, B02, and B03 were obtained during September 2002 just before the facility personnel were relocated to the Cheyenne Facility. The different blank corrections (BC) reflect different biases in low-level measurements found in blank data generated by two analytical instruments.

The low ProbPlot $\mathrm{R}$ value reflects the three anomalous observations visible in the plot. In spite of this value, the low PLall and very low UTL, along with the fact that the highest of the anomalous observations comes from a location that would arguably not be considered a normal part of the "touchable" work environment, suggests that there is no cause for concern with this facility.

Alternately, with over 59 IWAs sampled, one could simply rely on the NPUTL which is the largest IWA maximum value.

\section{4-B04}

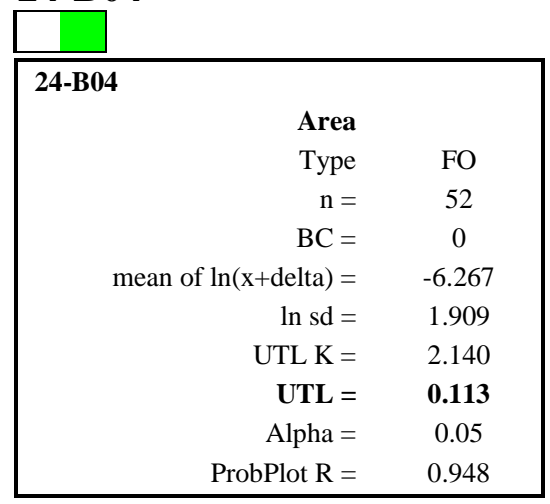

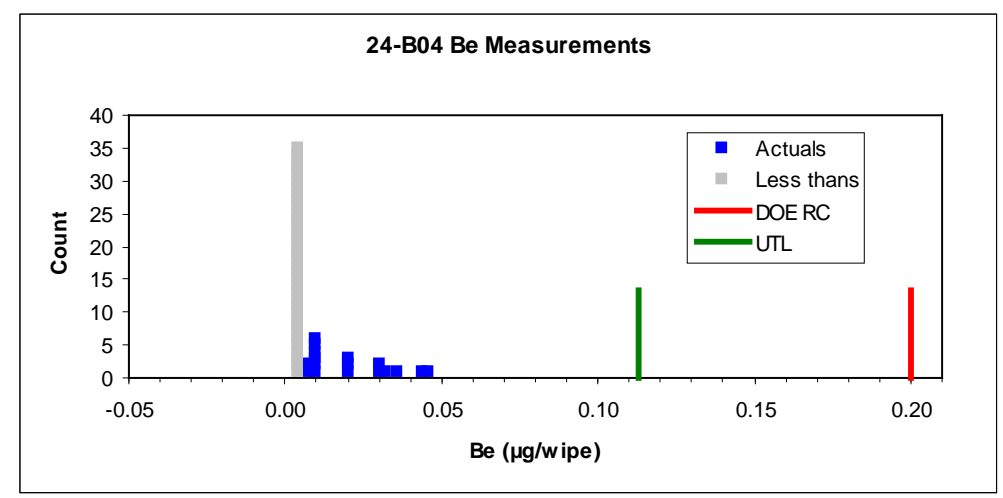




\section{4-B05}

\begin{tabular}{|c|c|}
\hline \multicolumn{2}{|l|}{ 24-B05 } \\
\hline Area & \\
\hline Type & OF \\
\hline $\mathrm{n}=$ & 14 \\
\hline $\mathrm{BC}=$ & 0 \\
\hline mean of $\ln (x+$ delta $)=$ & -5.404 \\
\hline $\ln \mathrm{sd}=$ & 0.729 \\
\hline UTL K = & 3.015 \\
\hline UTL = & 0.041 \\
\hline Alpha $=$ & 0.05 \\
\hline ProbPlot $\mathrm{R}=$ & 0.951 \\
\hline
\end{tabular}

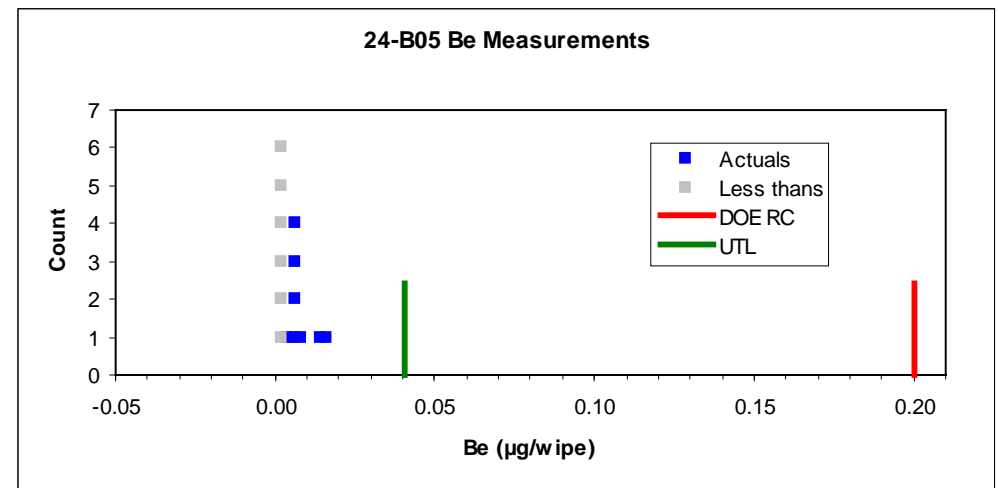

Prior data were used with these two facilities. We assume that the sampling plans at least reasonably approximated a systematic sampling plan, and note the low values obtained. The ProbPlot R values are based on “detects” only.

\section{4-B07}

\begin{tabular}{|c|c|}
\hline \multirow{2}{*}{\multicolumn{2}{|c|}{ 24-B07 }} \\
\hline & \\
\hline Type & SH \\
\hline $\mathrm{N}=$ & 24 \\
\hline $\mathrm{n}=$ & 10 \\
\hline $\mathrm{BC}(\mathrm{DC})=$ & 0.0003 \\
\hline delta $=$ & 0.0030 \\
\hline mean of $\ln (\mathrm{x}+$ delta $)=$ & -5.869 \\
\hline $\ln \mathrm{sd}=$ & 0.605 \\
\hline PL K = & 3.629 \\
\hline PLall $=$ & 0.022 \\
\hline UTL K = & 3.022 \\
\hline UTL = & 0.015 \\
\hline Alpha $=$ & 0.04 \\
\hline ProbPlot $\mathrm{R}=$ & 0.967 \\
\hline
\end{tabular}

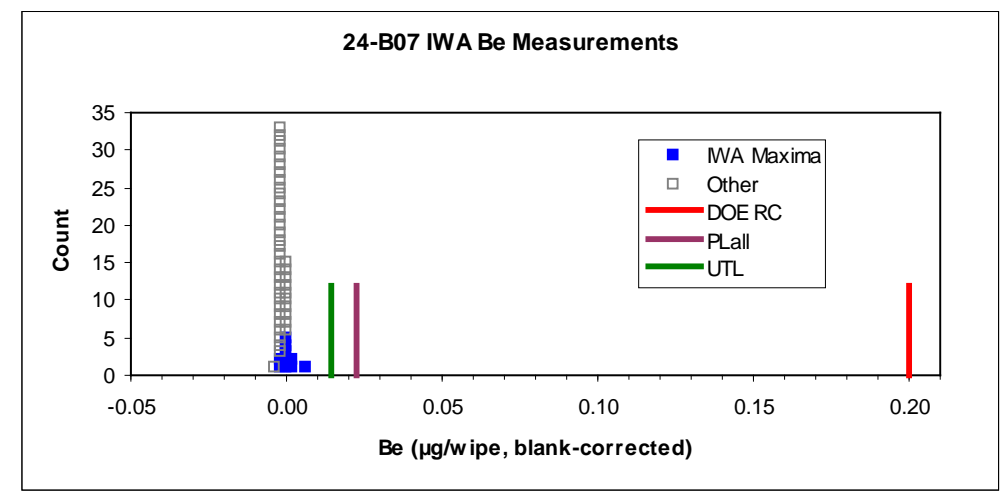

\begin{tabular}{|rc|}
\hline 24-B07 & \\
Areas & \\
Type & SH \\
$\mathrm{n}=$ & 36 \\
BC $(\mathrm{DC})=$ & 0.0003 \\
delta $=$ & 0.0055 \\
mean of $\ln (\mathrm{x}+$ delta $)=$ & -5.392 \\
$\ln \mathrm{sd}=$ & 0.166 \\
UTL $\mathrm{K}=$ & 2.415 \\
$\mathrm{UTL}=$ & $\mathbf{0 . 0 0 1}$ \\
Alpha $=$ & 0.01 \\
ProbPlot $\mathrm{R}=$ & 0.966 \\
\hline
\end{tabular}

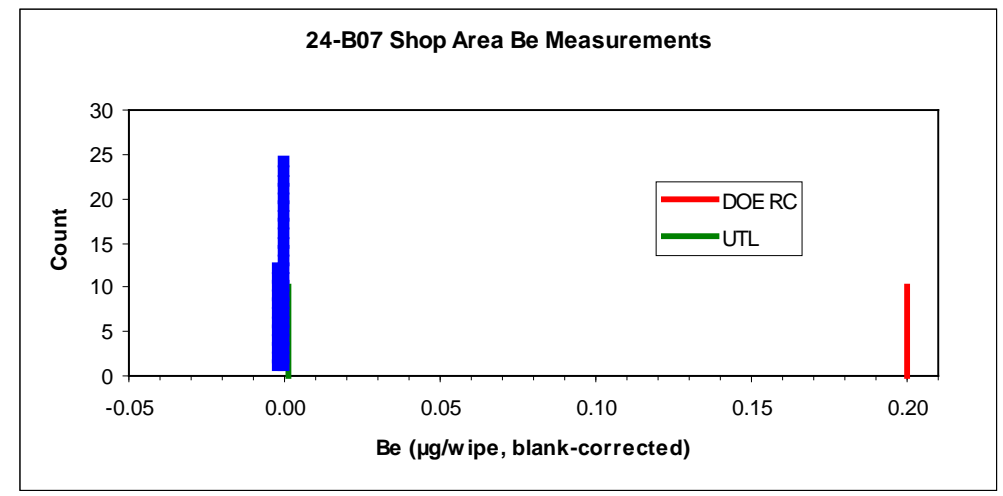




\section{4-C01}

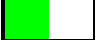

24-C01

\begin{tabular}{rc} 
IWAs & \\
Type & OF \\
$\mathrm{N}=$ & 521 \\
$\mathrm{n}=$ & 94 \\
BC $($ BNadj $)=$ & -0.0062 \\
delta $=$ & 0.0050 \\
mean of $\ln (\mathrm{x}+$ delta $)=$ & -4.887 \\
$\ln \mathrm{sd}=$ & 0.323 \\
PL $\mathrm{K}=$ & 3.827 \\
PLall $=$ & $\mathbf{0 . 0 2 1}$ \\
UTL $\mathrm{K}=$ & 1.937 \\
UTL $=$ & $\mathbf{0 . 0 0 9}$ \\
Alpha $=$ & 0.05 \\
ProbPlot $\mathrm{R}=$ & 0.891 \\
\hline
\end{tabular}

\section{4-C02}

\begin{tabular}{|c|c|c|}
\hline \multirow{2}{*}{\multicolumn{3}{|c|}{ 24-C02 }} \\
\hline & & \\
\hline & Туре & OF \\
\hline & $\mathrm{N}=$ & 29 \\
\hline & $\mathrm{n}=$ & 10 \\
\hline & $\mathrm{BC}(\mathrm{DC})=$ & 0.0003 \\
\hline & delta $=$ & 0.0050 \\
\hline & mean of $\ln (x+$ delta $)=$ & -4.836 \\
\hline & $\ln s d=$ & 0.254 \\
\hline & PL K = & 3.801 \\
\hline & PLall = & 0.016 \\
\hline & UTL K = & 3.022 \\
\hline & $\mathbf{U T L}=$ & 0.012 \\
\hline & Alpha = & 0.04 \\
\hline & ProbPlot $\mathrm{R}=$ & 0.954 \\
\hline
\end{tabular}

\begin{tabular}{|c|c|}
\hline \multicolumn{2}{|l|}{ Storage area 6204} \\
\hline Type & ST \\
\hline $\mathrm{n}=$ & 20 \\
\hline $\mathrm{BC}(\mathrm{DC})=$ & 0.0000 \\
\hline delta $=$ & 0.0055 \\
\hline mean of $\ln (x+$ delta $)=$ & -4.970 \\
\hline $\ln \mathrm{sd}=$ & 0.097 \\
\hline UTL K = & 2.808 \\
\hline UTL = & 0.004 \\
\hline Alpha $=$ & 0.01 \\
\hline ProbPlot $\mathrm{R}=$ & 0.980 \\
\hline
\end{tabular}

Worker Environment Beryllium Characterization Study 74 Appendix 2

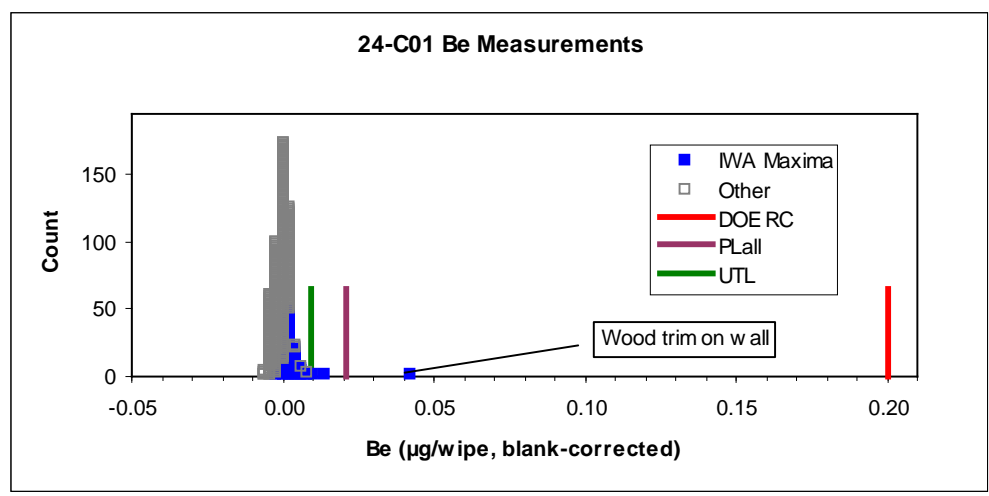

The low ProbPlot $\mathrm{R}$ is due to the outlier.
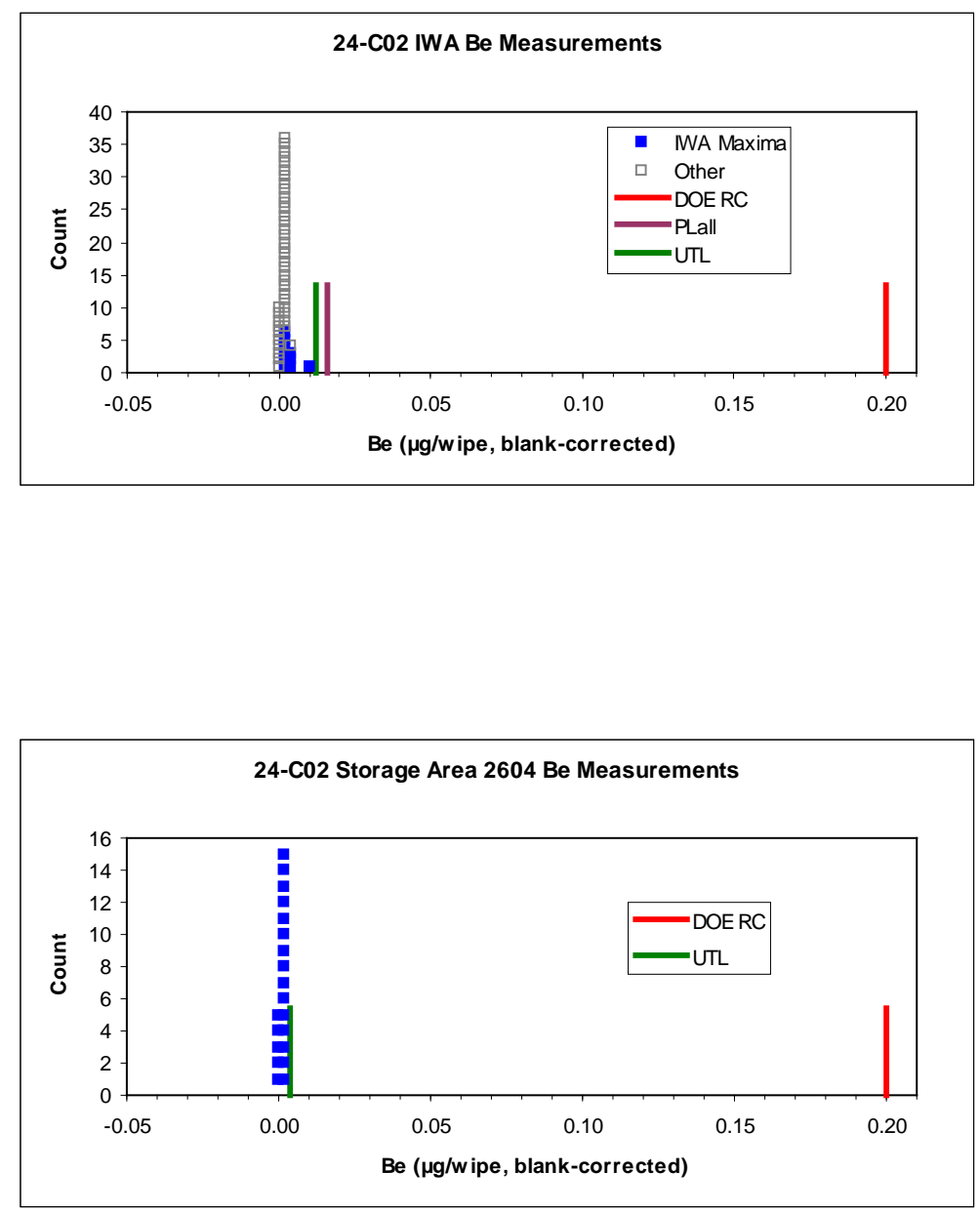

Revision 2.5

December 2009 


\section{4-C03}

$\square$

\begin{tabular}{|rc|}
\hline 24-C03 & \\
IWAs & \\
Type & OF \\
$\mathrm{N}=$ & 22 \\
$\mathrm{n}=$ & 11 \\
BC(DC) $=$ & 0.0003 \\
delta $=$ & 0.0050 \\
mean of $\ln (\mathrm{x}+$ delta $)=$ & -4.746 \\
$\mathrm{IL}=$ & 0.433 \\
PL $\mathrm{K}=$ & 3.258 \\
PLall $=$ & $\mathbf{0 . 0 3 1}$ \\
UTL K $=$ & 2.815 \\
UTL $=$ & $\mathbf{0 . 0 2 4}$ \\
Alpha $=$ & 0.05 \\
ProbPlot $\mathrm{R}=$ & 0.937 \\
\hline
\end{tabular}

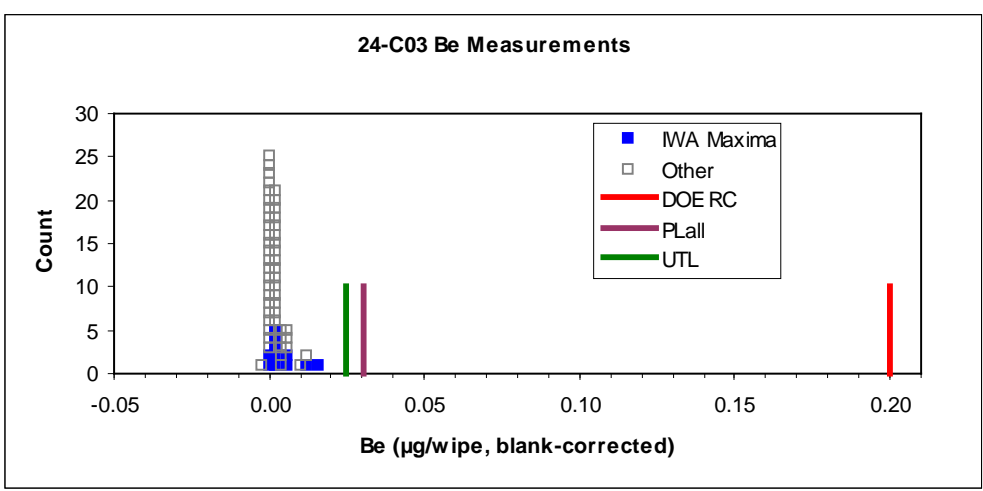

\section{4-NSF}

\begin{tabular}{|c|c|}
\hline \multicolumn{2}{|l|}{ 24-NSF } \\
\hline IWAs & \\
\hline $\mathrm{N}=$ & 687 \\
\hline $\mathrm{n}=$ & 43 \\
\hline $\mathrm{BC}(\mathrm{BNadj})=$ & -0.0062 \\
\hline $\mathrm{BC}(\mathrm{DC})=$ & 0.0013 \\
\hline delta $=$ & 0.0050 \\
\hline mean of $\ln (x+$ delta $)=$ & -4.750 \\
\hline $\ln \mathrm{sd}=$ & 0.353 \\
\hline PL K = & 4.138 \\
\hline PLall = & 0.032 \\
\hline UTL K = & 2.105 \\
\hline UTL = & 0.013 \\
\hline Alpha $=$ & 0.05 \\
\hline ProbPlot $\mathrm{R}=$ & 0.959 \\
\hline
\end{tabular}

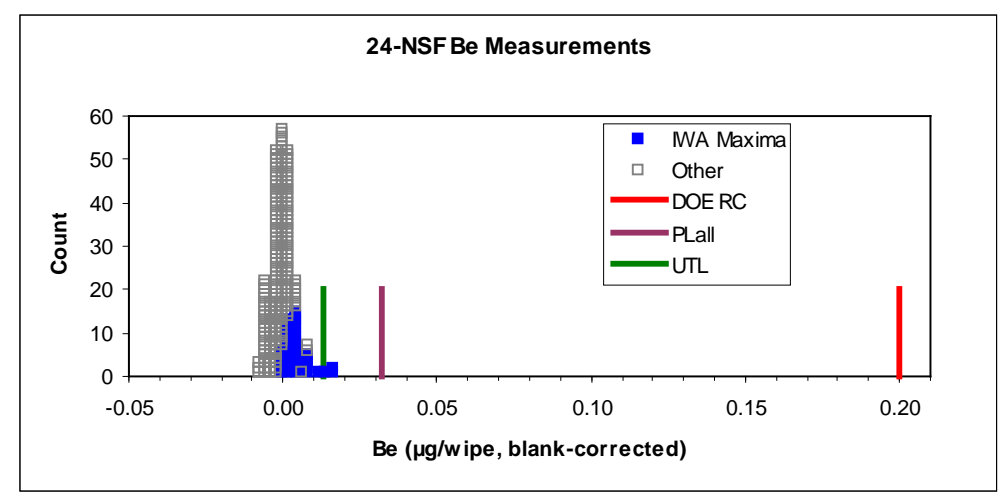




\section{5-202616}

\begin{tabular}{|c|c|}
\hline \multicolumn{2}{|l|}{$25-202616$} \\
\hline Area & \\
\hline Type & FO \\
\hline $\mathrm{n}=$ & 18 \\
\hline $\mathrm{BC}(\mathrm{DC})=$ & 0.0003 \\
\hline $\mathrm{BC}(\mathrm{BNadj})=$ & -0.0062 \\
\hline BC(MS_new) = & 0.0001 \\
\hline delta $=$ & 0.0085 \\
\hline mean of $\ln (\mathrm{x}+$ delta $)=$ & -3.670 \\
\hline $\ln s d=$ & 1.165 \\
\hline UTL K = & 2.453 \\
\hline UTL $=$ & 0.440 \\
\hline Alpha $=$ & 0.05 \\
\hline ProbPlot $\mathrm{R}=$ & 0.971 \\
\hline
\end{tabular}

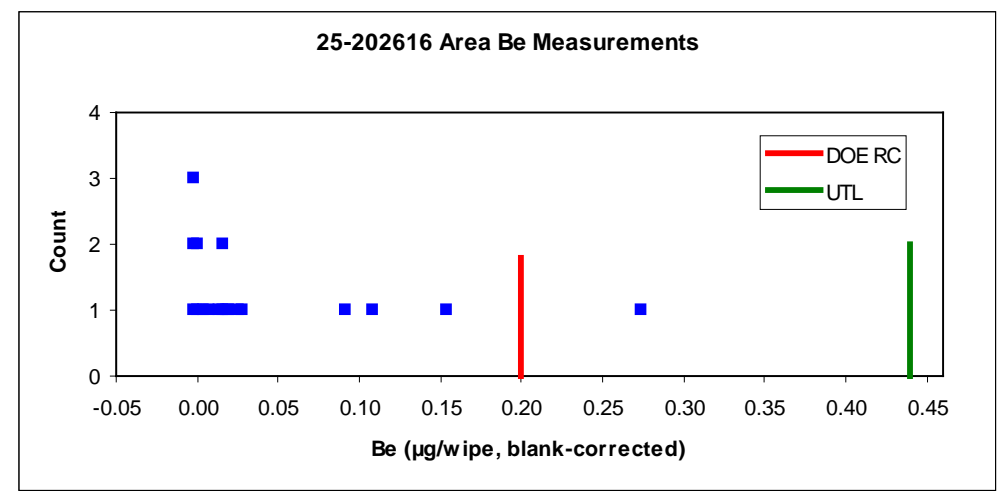

The metal ratios in the highest wipe are consistent with those in NTS soils.

\section{5-4117}

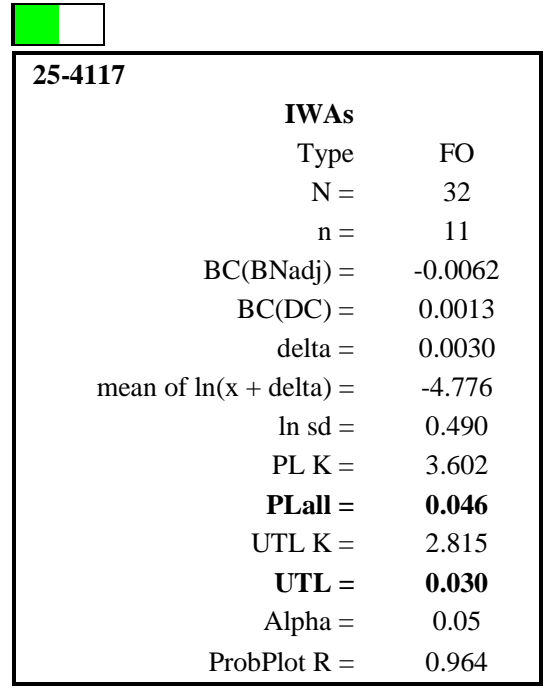

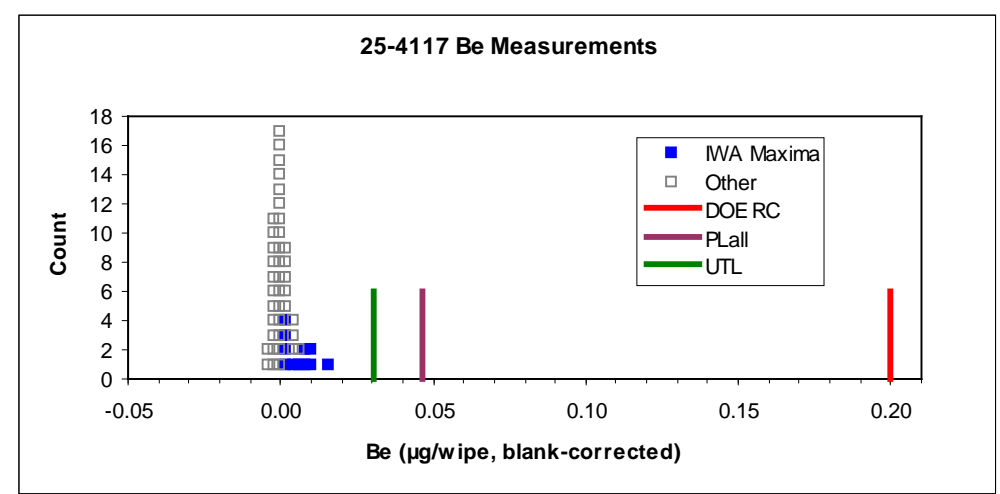




\section{5-4919}

\begin{tabular}{|c|c|}
\hline \multicolumn{2}{|l|}{$25-4919$} \\
\hline IWAs & \\
\hline Type & FO \\
\hline $\mathrm{N}=$ & 10 \\
\hline $\mathrm{n}=$ & 5 \\
\hline $\mathrm{BC}(\mathrm{BNadj})=$ & -0.0062 \\
\hline $\mathrm{BC}(\mathrm{DC})=$ & 0.0013 \\
\hline BC(MS_arch) = & 0.0004 \\
\hline delta $=$ & 0.0030 \\
\hline mean of $\ln (x+$ delta $)=$ & -4.472 \\
\hline $\ln \mathrm{sd}=$ & 1.231 \\
\hline $\mathrm{PL} \mathrm{K}=$ & 3.788 \\
\hline PLall $=$ & 1.208 \\
\hline UTL K = & 4.203 \\
\hline UTL = & 2.014 \\
\hline Alpha $=$ & 0.05 \\
\hline ProbPlot $\mathrm{R}=$ & 0.998 \\
\hline \multicolumn{2}{|c|}{ UTL cluster sampling analysis } \\
\hline $\mathrm{F}=$ & 9.76 \\
\hline delta $=$ & 0.0090 \\
\hline mean of $\ln (x+$ delta $)=$ & -4.413 \\
\hline $\operatorname{tau}^{\wedge} 2=$ & 0.392 \\
\hline $\operatorname{sig} \wedge 2=$ & 0.179 \\
\hline $\ln s d=$ & 0.756 \\
\hline UTL K = & 3.538 \\
\hline $\mathbf{U T L}=$ & 0.173 \\
\hline ProbPlot $\mathrm{R}=$ & 0.967 \\
\hline
\end{tabular}

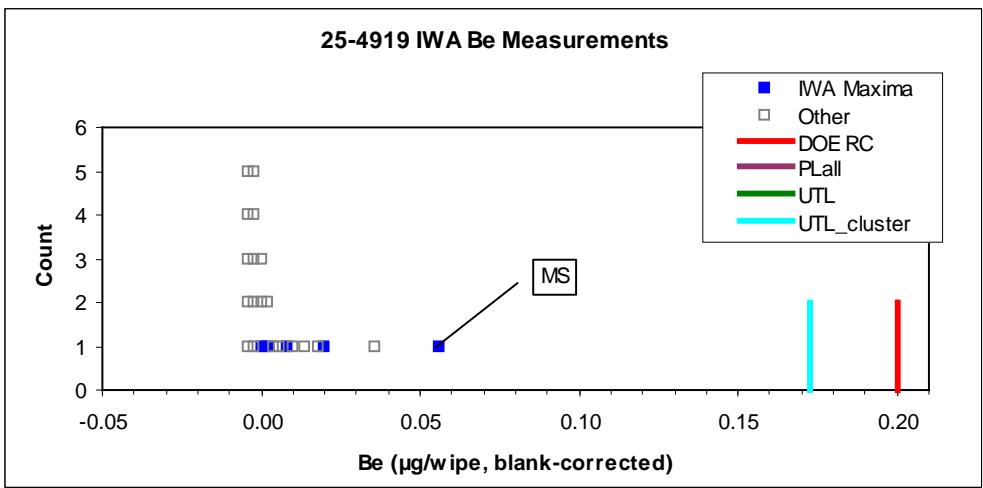

\section{6-2204}

\begin{tabular}{|c|c|}
\hline \multicolumn{2}{|l|}{$26-2204$} \\
\hline Area & \\
\hline Type & FO \\
\hline $\mathrm{n}=$ & 20 \\
\hline BC(MS_new $)=$ & 0.0001 \\
\hline delta $=$ & 0.0085 \\
\hline mean of $\ln (x+$ delta $)=$ & -3.582 \\
\hline $\ln s d=$ & 0.748 \\
\hline UTL K = & 2.396 \\
\hline UTL = & 0.166 \\
\hline Alpha $=$ & 0.05 \\
\hline ProbPlot $\mathrm{R}=$ & 0.967 \\
\hline
\end{tabular}

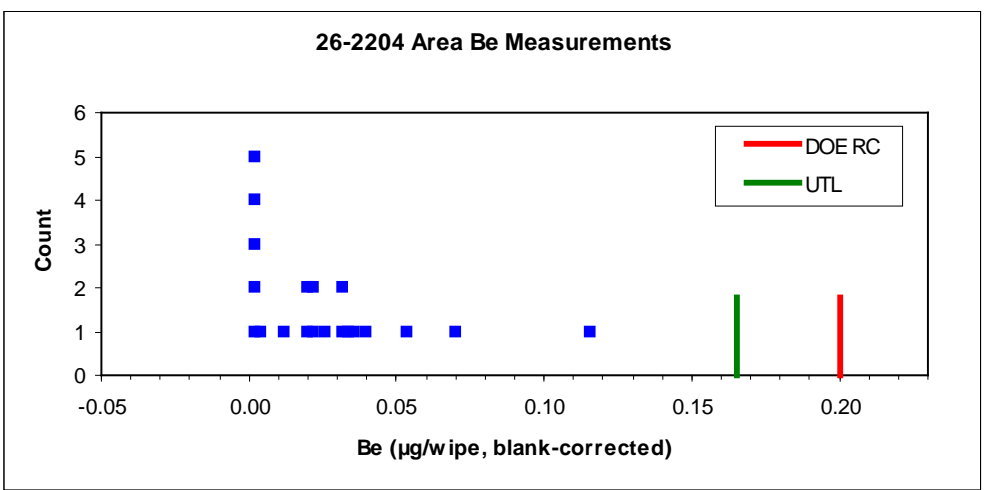

The Be concentration and metal ratios in the bulk sample obtained within the facility are consistent with NTS soils. 


\section{7-5100}

\begin{tabular}{|c|c|}
\hline \multirow{2}{*}{\multicolumn{2}{|c|}{$27-5100$}} \\
\hline & \\
\hline Type & EX \\
\hline $\mathrm{N}=$ & 5 \\
\hline $\mathrm{n}=$ & 4 \\
\hline $\mathrm{BC}(\mathrm{BNadj})=$ & -0.0062 \\
\hline $\mathrm{BC}(\mathrm{DC})=$ & 0.0013 \\
\hline delta $=$ & 0.0030 \\
\hline mean of $\ln (x+$ delta $)=$ & -5.052 \\
\hline $\ln \mathrm{sd}=$ & 0.672 \\
\hline $\mathrm{PL} \mathrm{K}=$ & 2.913 \\
\hline PLall $=$ & 0.042 \\
\hline UTL K = & 5.580 \\
\hline UTL = & 0.268 \\
\hline Alpha $=$ & 0.04 \\
\hline ProbPlot $\mathrm{R}=$ & 0.989 \\
\hline
\end{tabular}

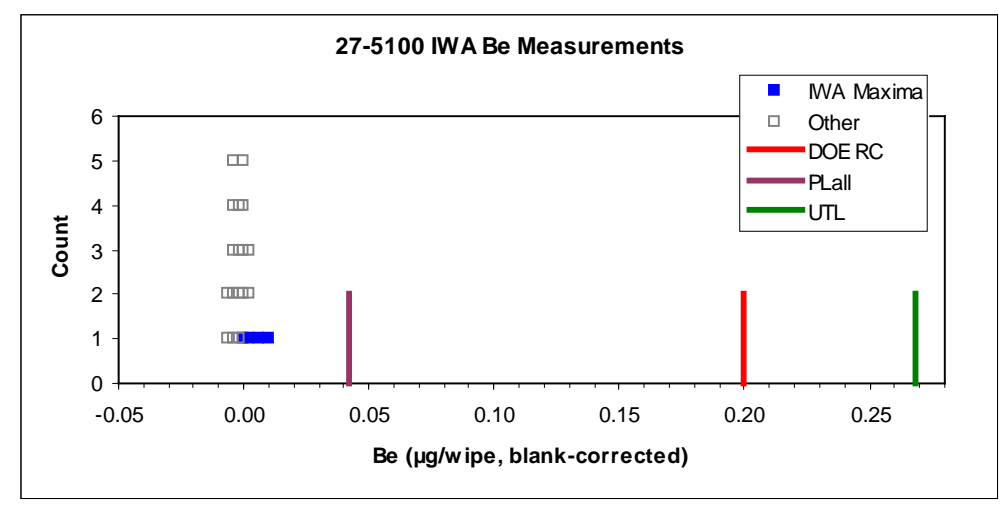

\begin{tabular}{|c|c|}
\hline \multirow{2}{*}{\multicolumn{2}{|c|}{$27-5100$}} \\
\hline & \\
\hline Туре & EX \\
\hline $\mathrm{n}=$ & 29 \\
\hline $\operatorname{shift}(\mathrm{BNadj})=$ & -0.0062 \\
\hline shift(DC) $=$ & 0.0013 \\
\hline delta $=$ & 0.0070 \\
\hline mean of $\ln (x+$ delta $)=$ & -5.818 \\
\hline $\ln \mathrm{sd}=$ & 1.028 \\
\hline UTL K = & 2.694 \\
\hline UTL = & 0.040 \\
\hline Alpha $=$ & 0.01 \\
\hline ProbPlot $\mathrm{R}=$ & 0.985 \\
\hline
\end{tabular}

Four of five IWAs were sampled, and PLall is well below the DOE RC. UTL is above; nonetheless the facility passes.

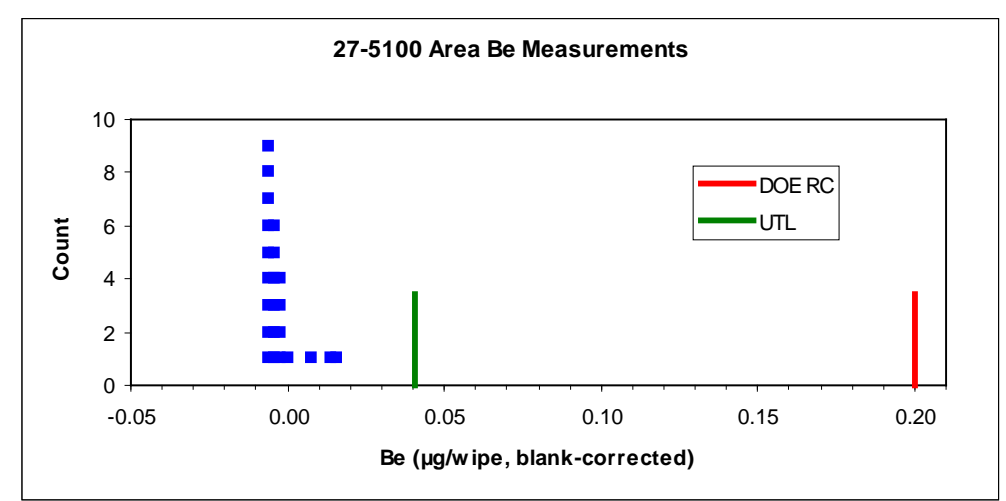




\section{7-5150}

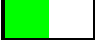

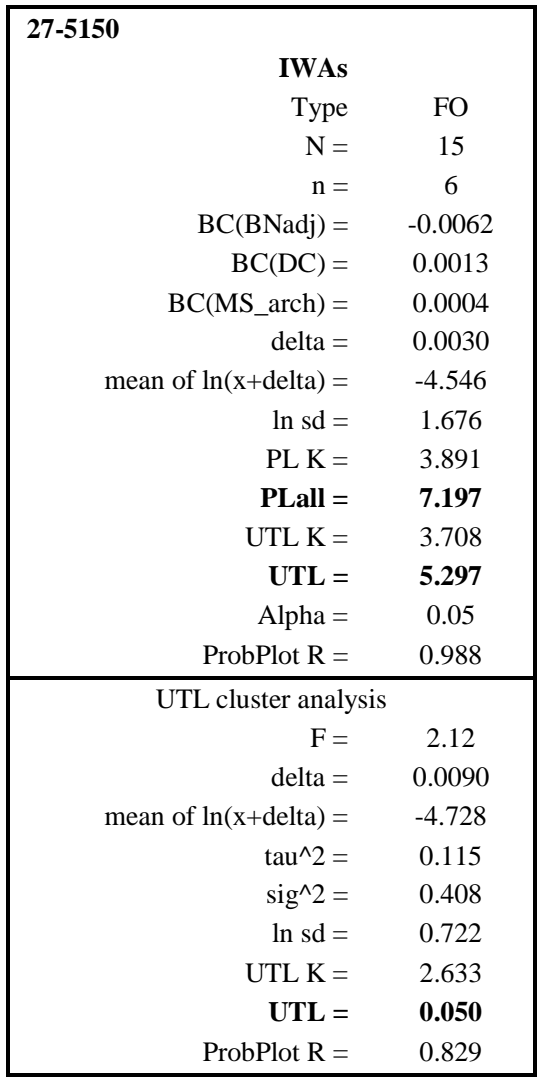

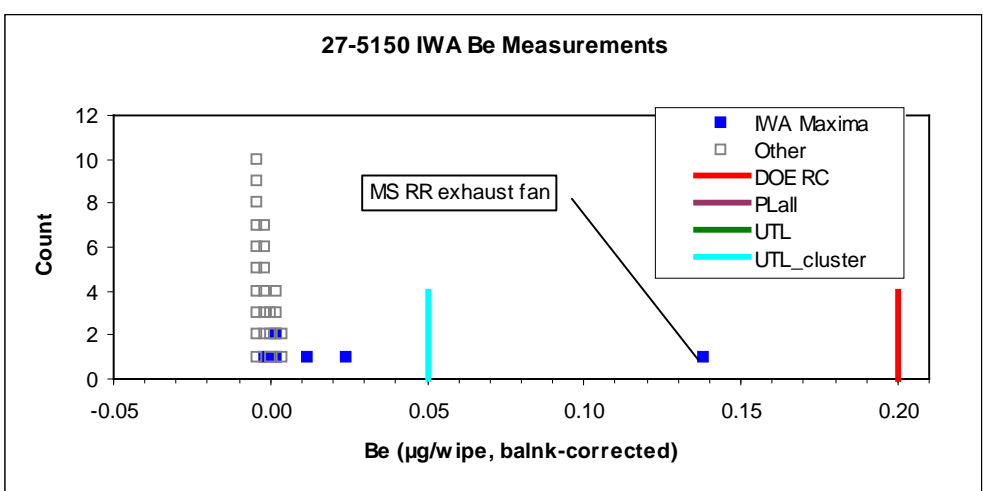

\section{7-5180}

\begin{tabular}{|c|c|}
\hline \multirow{2}{*}{\multicolumn{2}{|c|}{ 27-5180 }} \\
\hline & \\
\hline Туре & FO \\
\hline $\mathrm{n}=$ & 11 \\
\hline $\mathrm{BC}(\mathrm{BNadj})=$ & -0.0062 \\
\hline $\mathrm{BC}(\mathrm{DC})=$ & 0.0013 \\
\hline BC(MS_arch) $=$ & 0.0004 \\
\hline delta $=$ & 0.0080 \\
\hline mean of $\ln (x+$ delta $)=$ & -4.711 \\
\hline $\ln s d=$ & 0.564 \\
\hline UTL K = & 2.815 \\
\hline UTL = & 0.044 \\
\hline Alpha $=$ & 0.05 \\
\hline ProbPlot $\mathrm{R}=$ & 0.946 \\
\hline
\end{tabular}

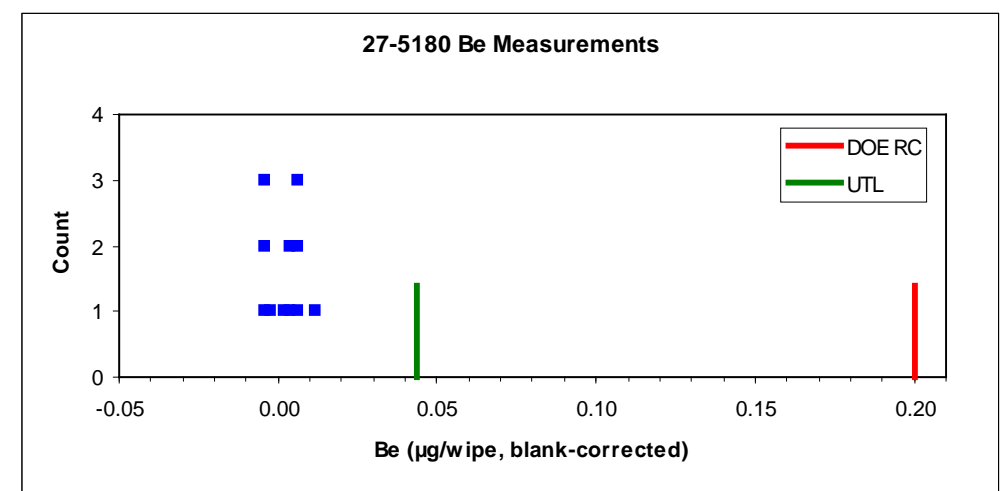




\section{7-5191}

\begin{tabular}{|c|c|}
\hline \multicolumn{2}{|l|}{ 27-5191 } \\
\hline \multicolumn{2}{|l|}{ Area } \\
\hline Tyре & $\mathrm{SH}$ \\
\hline $\mathrm{n}=$ & 14 \\
\hline $\mathrm{BC}(\mathrm{DC})=$ & 0.0013 \\
\hline $\mathrm{BC}(\mathrm{BNadj})=$ & -0.0062 \\
\hline BC(MS_arch $)=$ & 0.0004 \\
\hline delta $=$ & 0.008 \\
\hline mean of $\ln (x+$ delta $)=$ & -4.384 \\
\hline $\ln \mathrm{sd}=$ & 1.179 \\
\hline UTL K = & 2.614 \\
\hline UTL = & 0.264 \\
\hline Alpha $=$ & 0.05 \\
\hline ProbPlot $\mathrm{R}=$ & 0.877 \\
\hline \multicolumn{2}{|c|}{ omitting door top } \\
\hline mean of $\ln (x+$ delta $)=$ & -4.646 \\
\hline $\ln \mathrm{sd}=$ & 0.683 \\
\hline UTL K = & 2.671 \\
\hline UTL = & 0.051 \\
\hline ProbPlot $\mathrm{R}=$ & 0.948 \\
\hline
\end{tabular}

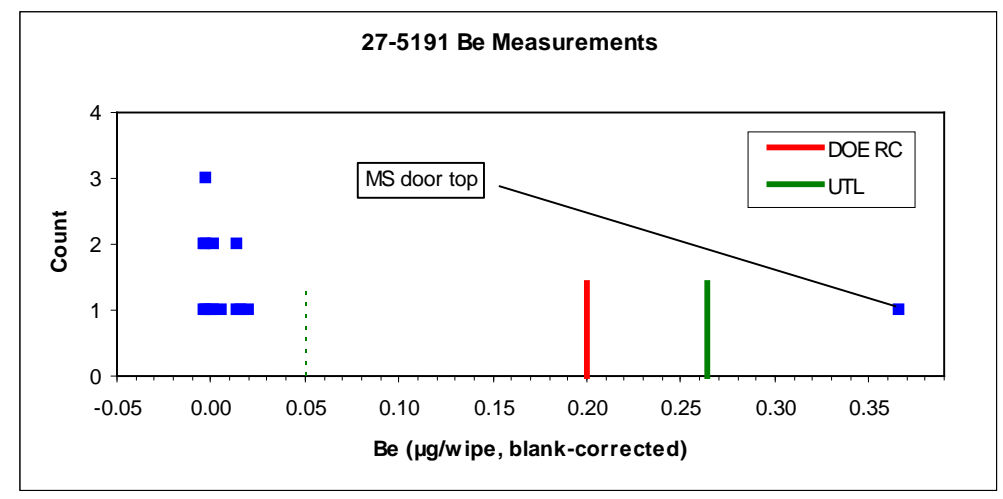

One isolated MS_arch measurement is above the DOE RC. Omitting the high measurement, obtained in a location not normally touchable during routine work, UTL is beneath the DOE RC.

\section{7-B}

\begin{tabular}{|c|c|c|}
\hline \multicolumn{3}{|l|}{ 27-B } \\
\hline & IWAs & \\
\hline & Type & FO \\
\hline & $\mathrm{N}=$ & 5 \\
\hline & $\mathrm{n}=$ & 3 \\
\hline & $\mathrm{BC}(\mathrm{BNadj})=$ & -0.0062 \\
\hline & $\mathrm{BC}(\mathrm{DC})=$ & 0.0013 \\
\hline & BC(MS_arch) = & 0.0004 \\
\hline & delta $=$ & 0.0030 \\
\hline & mean of $\ln (x+$ delta $)=$ & -4.239 \\
\hline & $\ln \mathrm{sd}=$ & 0.978 \\
\hline & PL K = & 4.572 \\
\hline & PLall = & 1.259 \\
\hline & UTL K = & 7.656 \\
\hline & UTL $=$ & 25.770 \\
\hline & Alpha = & 0.05 \\
\hline & ProbPlot $\mathrm{R}=$ & 0.961 \\
\hline \multicolumn{3}{|c|}{ UTL cluster analysis } \\
\hline & $\mathrm{F}=$ & 0.26 \\
\hline & delta $=$ & 0.0090 \\
\hline & mean of $\ln (x+$ delta $)=$ & -4.519 \\
\hline & $\ln \mathrm{sd}=$ & 0.637 \\
\hline & UTL K = & 2.736 \\
\hline & UTL = & 0.053 \\
\hline & ProbPlot R = & 0.954 \\
\hline
\end{tabular}

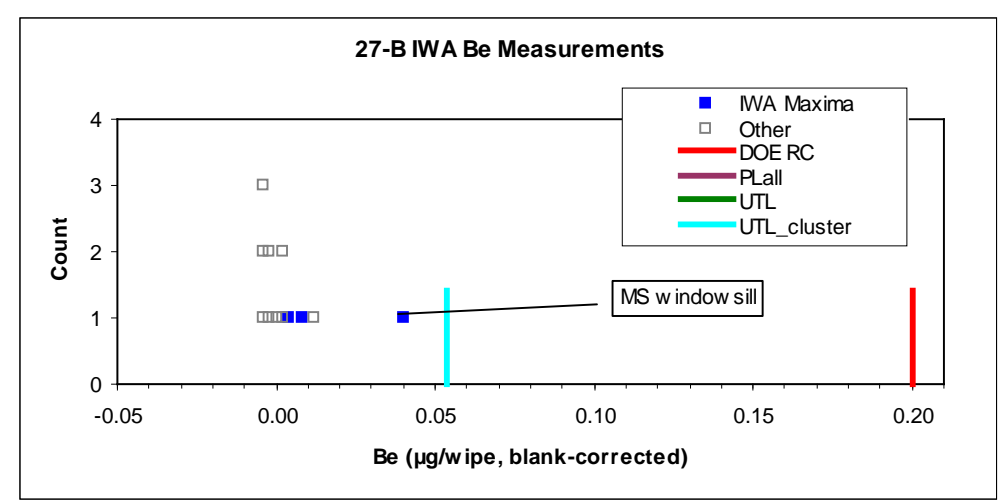




\section{5-2211}

\begin{tabular}{|rc|}
\hline & \\
\hline 35-2211 & \\
IWAs & \\
Type & OF \\
$\mathrm{N}=$ & 358 \\
$\mathrm{n}=$ & 87 \\
BC(DC) $=$ & 0.0003 \\
delta $=$ & 0.0050 \\
mean of $\ln (\mathrm{x}+$ delta $)=$ & -4.646 \\
$\ln \mathrm{sd}=$ & 0.366 \\
PL K $=$ & 3.777 \\
PLall $=$ & $\mathbf{0 . 0 3 3}$ \\
UTL K $=$ & 1.971 \\
UTL $=$ & $\mathbf{0 . 0 1 5}$ \\
Alpha $=$ & 0.04 \\
ProbPlot $\mathrm{R}=$ & 0.923
\end{tabular}

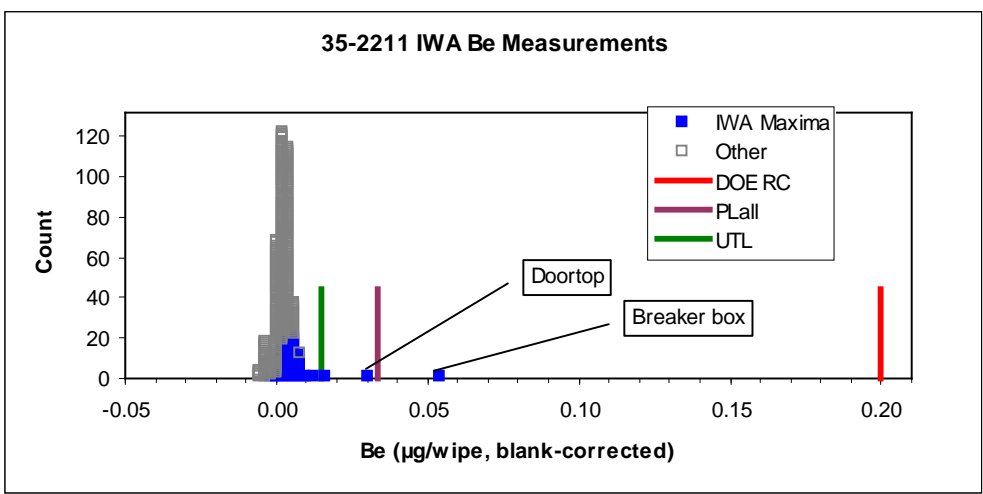

\begin{tabular}{|rc|}
\hline 35-2211 & \\
Hanger area & \\
Type & FO \\
$\mathrm{n}=$ & 31 \\
BC(DC) $=$ & 0.0003 \\
delta $=$ & 0.0055 \\
mean of $\ln (\mathrm{x}+$ delta $)=$ & -5.111 \\
$\ln \mathrm{sd}=$ & 0.191 \\
UTL $\mathrm{K}=$ & 2.496 \\
$\mathrm{UTL}=$ & $\mathbf{0 . 0 0 4}$ \\
Alpha $=$ & 0.01 \\
ProbPlot $\mathrm{R}=$ & 0.995 \\
\hline
\end{tabular}

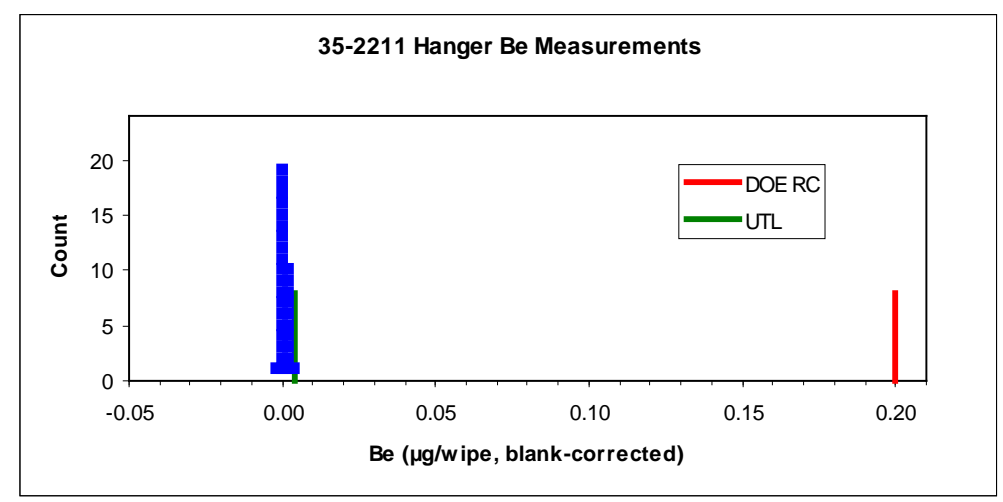

\section{5-2216}

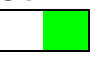

\begin{tabular}{|c|c|}
\hline \multicolumn{2}{|l|}{$35-2216$} \\
\hline Area & \\
\hline Type & ST \\
\hline $\mathrm{n}=$ & 32 \\
\hline $\mathrm{BC}(\mathrm{DC})=$ & 0.0003 \\
\hline delta $=$ & 0.0055 \\
\hline mean of $\ln (x+$ delta $)=$ & -4.273 \\
\hline $\ln \mathrm{sd}=$ & 0.534 \\
\hline UTL K = & 2.197 \\
\hline UTL = & 0.040 \\
\hline Alpha $=$ & 0.05 \\
\hline ProbPlot $\mathrm{R}=$ & 0.973 \\
\hline
\end{tabular}

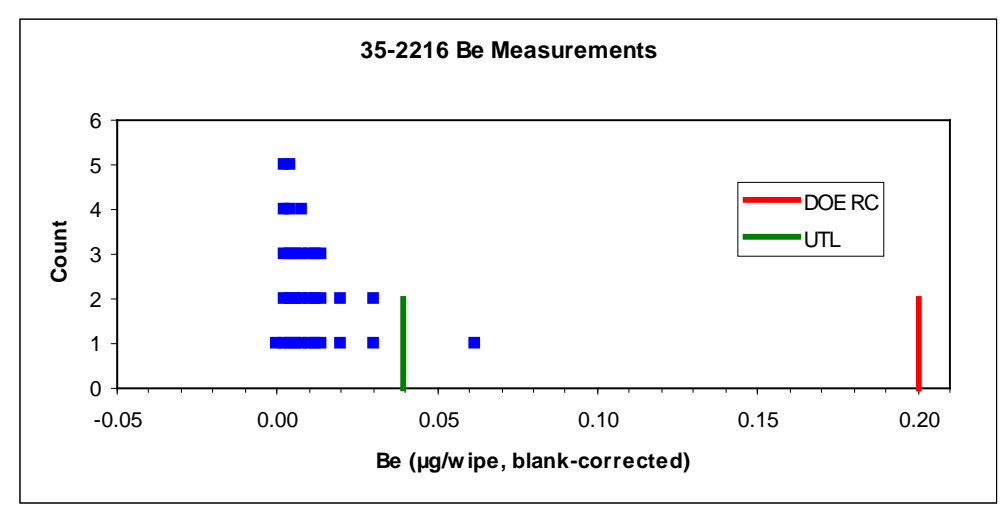




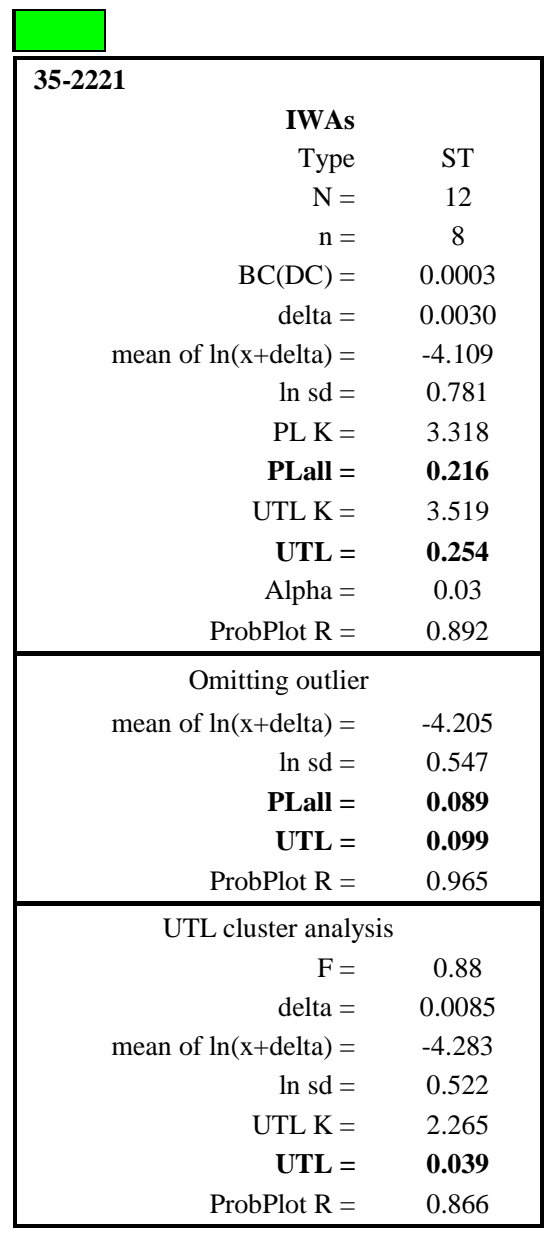

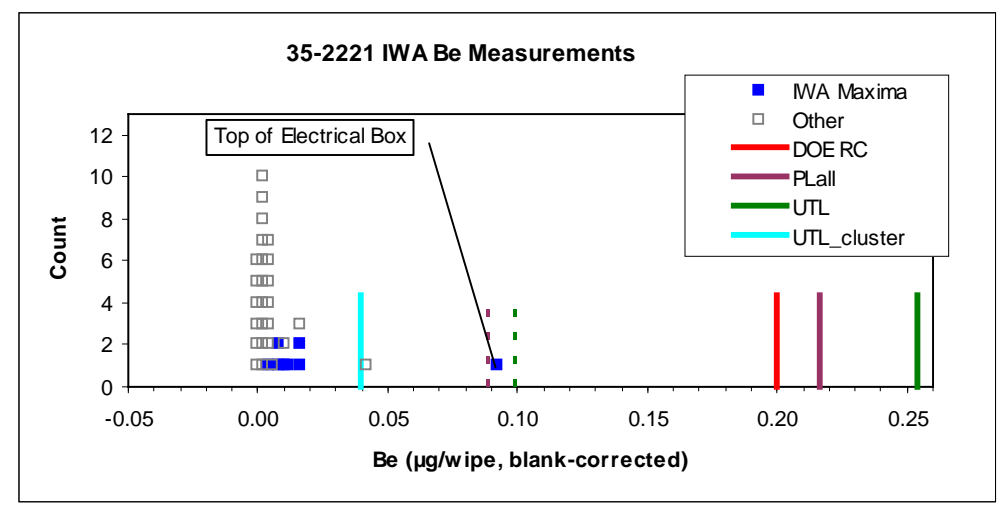

This facility is the Remote Sensing Laboratory (RSL)

Deployment Warehouse. There are 12 IWAs in the facility as well as numerous shelves containing equipment, much of it containerized. For analysis purposes three portions were used: the IWAs, the building proper, and the shelves and items on the shelves.

There is one outlier observation in the IWA portion, obtained atop an electrical box. The facility passes using the UTL cluster analysis with all data, not just the IWA maxima, or if the analysis is done omitting the high observation.

\begin{tabular}{|rc|}
\hline 35-2221 & \\
Building components & \\
Type & $\mathrm{ST}$ \\
$\mathrm{n}=$ & 14 \\
$\mathrm{BC}(\mathrm{DC})=$ & 0.0003 \\
delta $=$ & 0.0055 \\
mean of $\ln (\mathrm{x}+$ delta $)=$ & -4.474 \\
$\ln \mathrm{sd}=$ & 0.627 \\
$\mathrm{UTL} \mathrm{K}=$ & 3.189 \\
$\mathrm{UTL}=$ & $\mathbf{0 . 0 7 9}$ \\
Alpha $=$ & 0.01 \\
ProbPlot $\mathrm{R}=$ & 0.944 \\
\hline
\end{tabular}

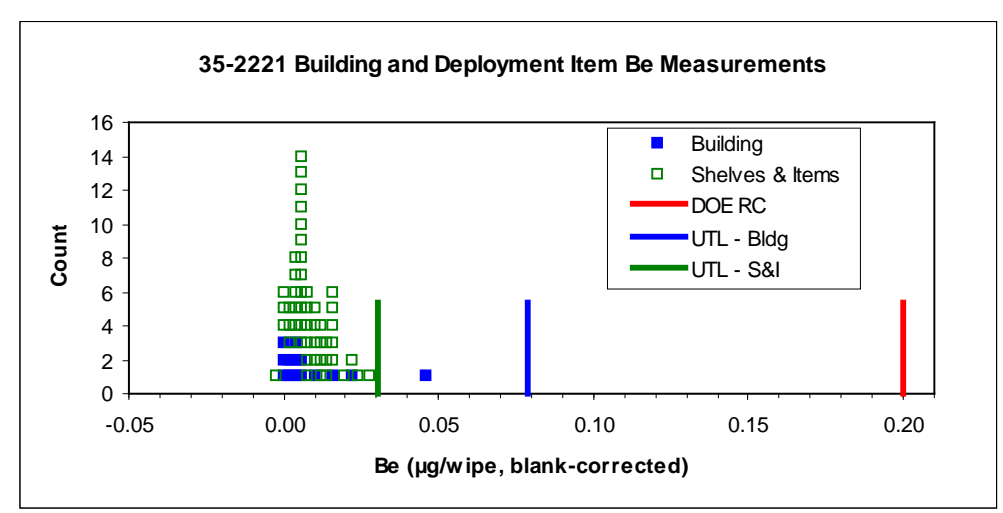




\begin{tabular}{|c|c|}
\hline \multirow{2}{*}{\multicolumn{2}{|c|}{ Shelves and items }} \\
\hline & \\
\hline Type & ST \\
\hline $\mathrm{n}=$ & 50 \\
\hline $\mathrm{BC}(\mathrm{DC})=$ & 0.0003 \\
\hline delta $=$ & 0.0055 \\
\hline mean of $\ln (x+$ delta $)=$ & -4.347 \\
\hline $\ln \mathrm{sd}=$ & 0.450 \\
\hline UTL K = & 2.269 \\
\hline UTL = & 0.030 \\
\hline Alpha $=$ & 0.01 \\
\hline ProbPlot $\mathrm{R}=$ & 0.995 \\
\hline
\end{tabular}

\section{5-2222}

\begin{tabular}{|c|c|}
\hline \multicolumn{2}{|l|}{$35-2222$} \\
\hline IWAs & \\
\hline Type & EX \\
\hline $\mathrm{N}=$ & 7 \\
\hline $\mathrm{n}=$ & 5 \\
\hline $\mathrm{BC}(\mathrm{DC})=$ & 0.0003 \\
\hline delta $=$ & 0.0020 \\
\hline mean of $\ln (x+$ delta $)=$ & -4.354 \\
\hline $\ln \mathrm{sd}=$ & 0.857 \\
\hline PL K = & 2.952 \\
\hline PLall $=$ & 0.159 \\
\hline UTL K = & 4.203 \\
\hline UTL = & 0.469 \\
\hline Alpha $=$ & 0.05 \\
\hline ProbPlot $\mathrm{R}=$ & 0.975 \\
\hline
\end{tabular}

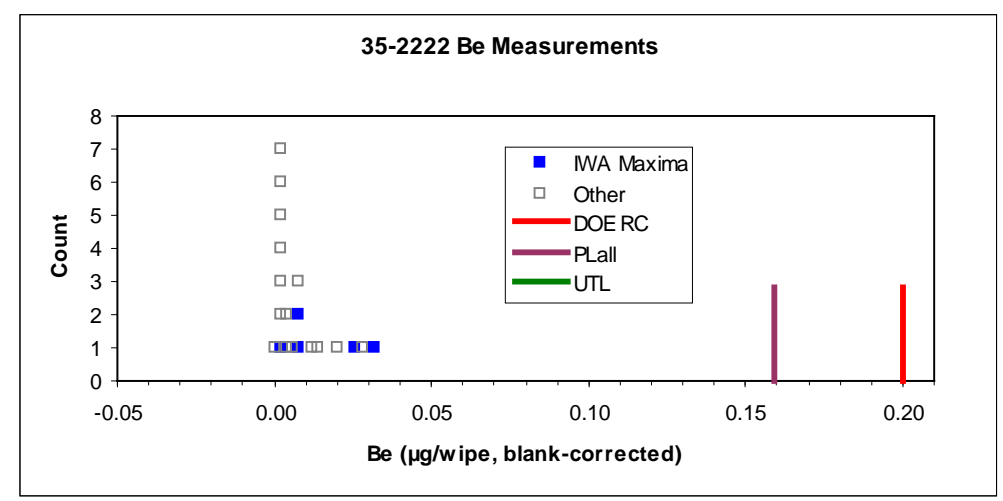

The UTL is much higher than the PLall for this small facility. The latter is well below the DOE $\mathrm{RC}$, though, so the facility passes.

\section{5-2229}

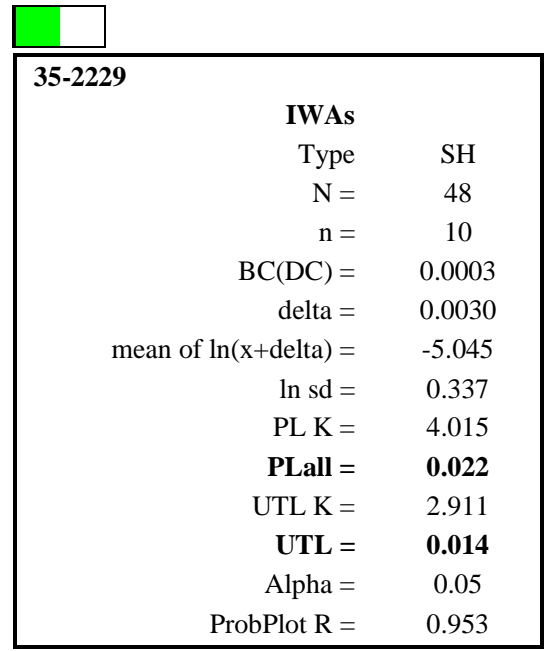

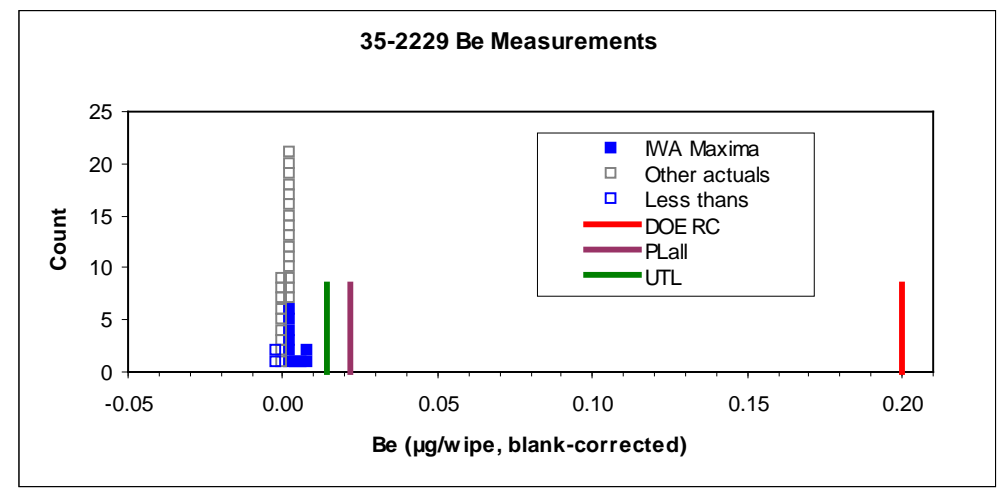


37-FG3 and FG4 (Cheyenne Facility)

\begin{tabular}{|c|c|}
\hline \multirow{2}{*}{\multicolumn{2}{|c|}{ 37-FG3\&4 }} \\
\hline & \\
\hline Type & OF \\
\hline $\mathrm{N}=$ & 198 \\
\hline $\mathrm{n}=$ & 9 \\
\hline $\mathrm{BC}(\mathrm{DC})=$ & 0.0003 \\
\hline $\mathrm{sd}=$ & 0.0050 \\
\hline mean of $\ln (x+$ delta $)=$ & -5.122 \\
\hline $\ln \mathrm{sd}=$ & 0.280 \\
\hline $\mathrm{PL} \mathrm{K}=$ & 6.190 \\
\hline PLall $=$ & 0.029 \\
\hline UTL $\mathrm{K}=$ & 3.754 \\
\hline UTL = & 0.012 \\
\hline Alpha $=$ & 0.05 \\
\hline ProbPlot $\mathrm{R}=$ & 0.958 \\
\hline
\end{tabular}

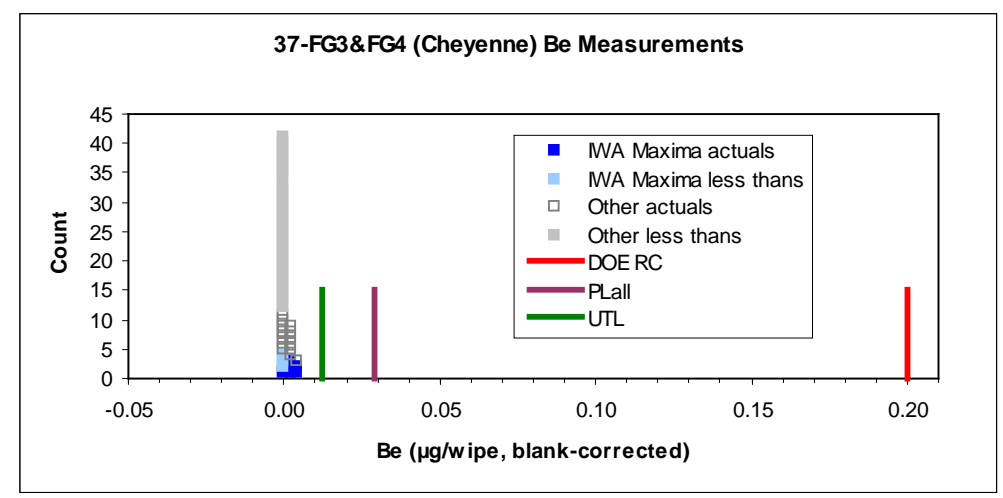

\section{BN-LAO}

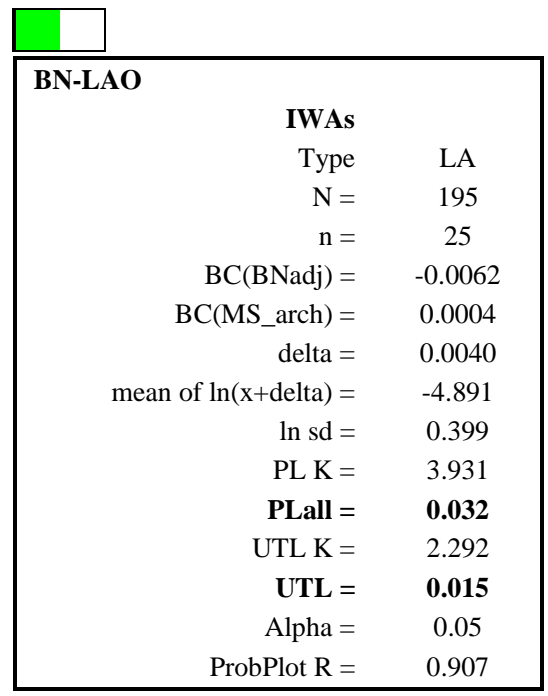

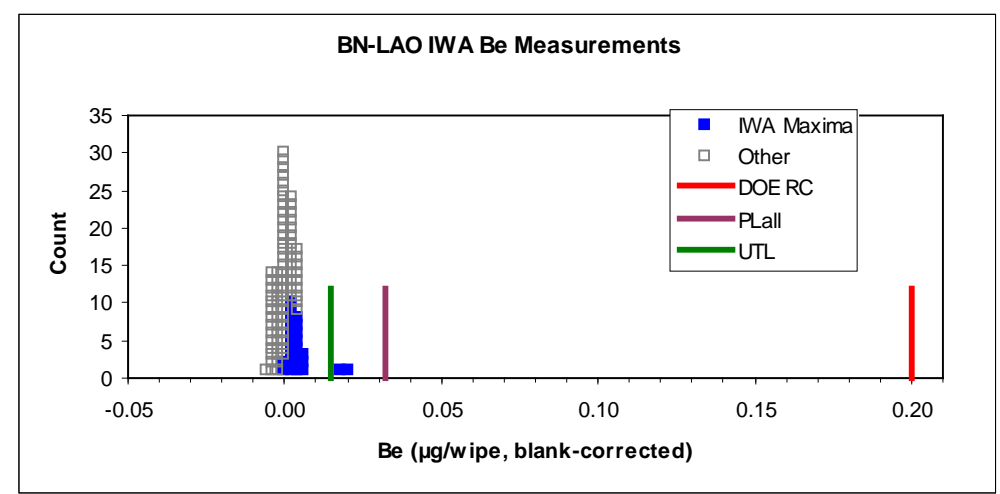




\section{BN-LLO}

\begin{tabular}{|c|c|}
\hline \multicolumn{2}{|l|}{ BN-LLO } \\
\hline IWAs & \\
\hline Type & EX \\
\hline $\mathrm{N}=$ & 114 \\
\hline $\mathrm{n}=$ & 12 \\
\hline $\mathrm{BC}(\mathrm{BNadj})=$ & -0.0062 \\
\hline $\mathrm{BC}(\mathrm{MS}(\operatorname{arch})=$ & 0.0004 \\
\hline delta $=$ & 0.0040 \\
\hline mean of $\ln (x+$ delta $)=$ & -4.829 \\
\hline $\ln s d=$ & 0.859 \\
\hline PL K = & 4.290 \\
\hline PLall = & 0.314 \\
\hline UTL K = & 2.736 \\
\hline UTL = & 0.080 \\
\hline Alpha $=$ & 0.05 \\
\hline ProbPlot R = & 0.839 \\
\hline
\end{tabular}

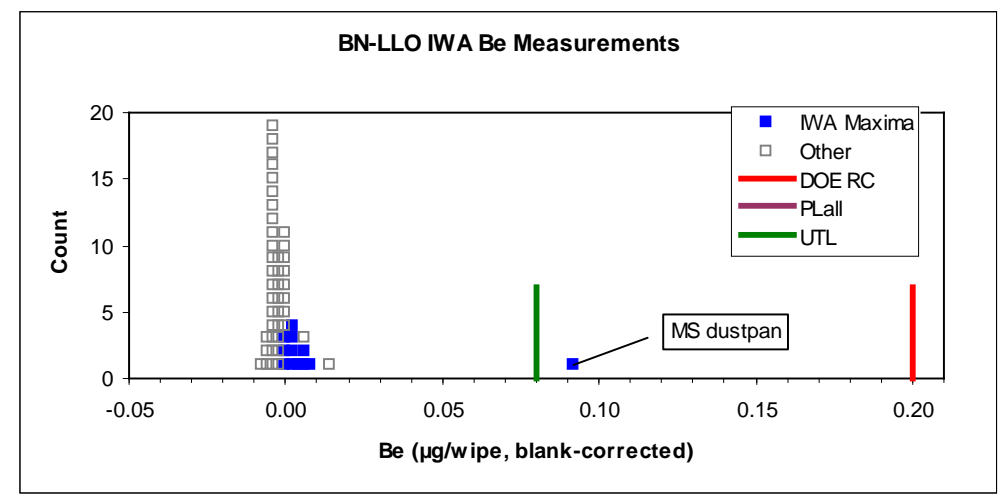

\section{BN-RSL Andrews}

\begin{tabular}{|c|c|}
\hline \multirow{2}{*}{\multicolumn{2}{|c|}{ BN-RSL Andrews }} \\
\hline & \\
\hline Type & FO \\
\hline $\mathrm{n}=$ & 27 \\
\hline $\mathrm{BC}(\mathrm{BNadj})=$ & -0.0062 \\
\hline BC(MS_arch $)=$ & 0.0004 \\
\hline delta $=$ & 0.0080 \\
\hline mean of $\ln (x+$ delta $)=$ & -4.526 \\
\hline $\ln \mathrm{sd}=$ & 0.584 \\
\hline UTL K = & 2.260 \\
\hline UTL $=$ & 0.040 \\
\hline Alpha $=$ & 0.05 \\
\hline ProbPlot $\mathrm{R}=$ & 0.913 \\
\hline
\end{tabular}

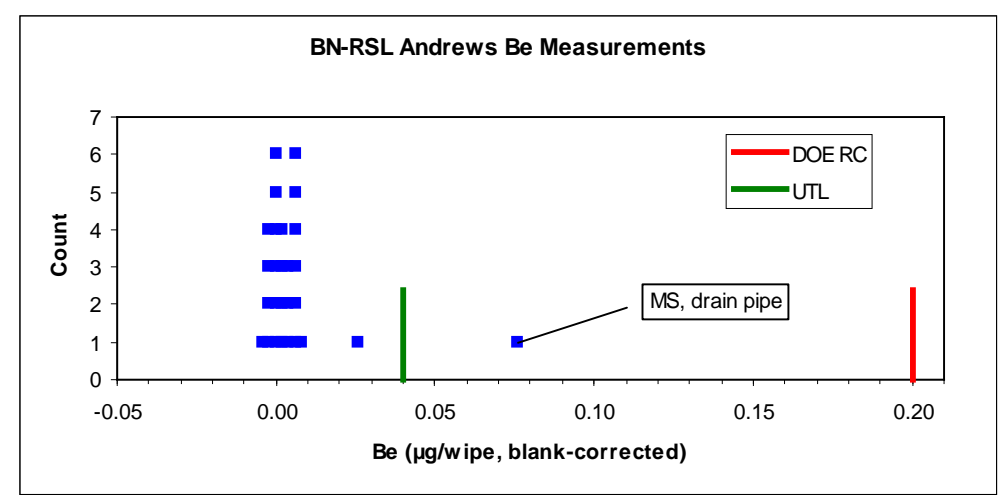

\section{Be and Metal Ratio Results from Bulk and High Wipe Samples}

During the January 2009 resampling, bulk samples were obtained from numerous facilities, along with exterior soil samples in the vicinities of those facilities. As discussed in Appendix 4, these were analyzed for Be and also for Co [cobalt], Ni [nickel], Cu [copper], Y [ytritium], $\mathrm{Nb}$ [niobium] and ${ }^{238} \mathrm{U}$ ([uranium-238]). From these data, 95\% central intervals were constructed for soil Be and for the logs of the ratios $\mathrm{Cu} / \mathrm{Be}$ and so on; plots of the soil data and log ratios are given in Appendix 4.

The first table below lists Be values and coded log metal ratio data for the bulk samples. The Be values are in their original units. The log metal ratio data are in coded units: the median log ratio of the soil data is coded to 12, with each unit from that representing a decrease or an increase of $25 \%$. This coding allows us to view the ratios for the different metals on a more-or-less common scale. Recall, however, that the ratios for $\mathrm{Y} / \mathrm{Be}$ (especially), Nb/Be, and U/Be are much more consistent in NTS soils than those for the other three metals and, hence, should be taken as more reliable indicators that a particular sample resembles NTS soils in its chemical fingerprint. 
Cells highlighted in green have Be values or metal ratios with relatively less Be than NTS soils. Cells highlighted in rose have metal ratios with relatively more Be than NTS soils. The value

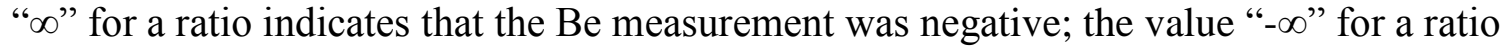
involving $\mathrm{Ni}$ indicates that the $\mathrm{Ni}$ measurement is reported as zero.

There are no bulk samples with relatively higher Be than NTS soils. There are several with relatively less Be; presumably these samples contain major portions of materials other than soils. There is one sample with a relatively lower Y/Be ratio than NTS soils. In a sample obtained in 06-CP160, the ratio is only slightly lower than the "normal" range for NTS soils. The Nb/Be ratio is also somewhat low in another bulk sample from that facility.

\begin{tabular}{|c|c|c|c|c|c|c|c|}
\hline \multicolumn{8}{|c|}{ Bulk Sample (January 2009) Metal Ratio Results } \\
\hline \multirow[b]{3}{*}{ Facility } & Be & $\mathrm{Co} / \mathrm{Be}$ & $\mathrm{Ni} / \mathrm{Be}$ & $\mathrm{Cu} / \mathrm{Be}$ & Y/Be & $\mathbf{N b} / \mathrm{Be}$ & U/Be \\
\hline & & \multicolumn{6}{|c|}{ Coded values for $\log ($ ratio $)$} \\
\hline & $\mathrm{mg} / \mathrm{Kg}$ & \multicolumn{6}{|c|}{ Soil median $=12$; one unit $=25 \%$ change } \\
\hline Soil 2.5 percentile & 0.379 & 5.7 & 5.1 & 3.0 & 10.0 & 9.7 & 8.4 \\
\hline Soil Median & 1.122 & 12.0 & 12.0 & 12.0 & 12.0 & 12.0 & 12.0 \\
\hline Soil 97.5 percentile & 1.865 & 18.3 & 18.9 & 21.0 & 14.0 & 14.3 & 15.6 \\
\hline 01-201959 & 1.01 & 12.8 & 14.7 & 15.8 & 11.7 & 16.4 & 11.7 \\
\hline 01-202479 & 1.40 & 11.1 & 13.3 & 12.0 & 12.5 & 11.6 & 11.1 \\
\hline 03-3С36 & 1.73 & 9.9 & 9.3 & 8.5 & 11.2 & 13.1 & 10.3 \\
\hline $05-24$ & 1.09 & 10.2 & 6.2 & 8.2 & 11.9 & 13.7 & 11.2 \\
\hline $05-24$ & 1.17 & 9.9 & 8.3 & 9.2 & 11.3 & 12.7 & 11.3 \\
\hline 05-202177 & 1.18 & 14.9 & 15.1 & 14.2 & 12.2 & 12.3 & 11.4 \\
\hline 06-159 & 0.77 & 19.5 & 27.5 & 34.3 & 13.2 & 13.7 & 13.8 \\
\hline 06-159 & 0.77 & 17.3 & 21.5 & 28.6 & 12.3 & 13.2 & 13.6 \\
\hline 06-624 & 1.11 & 10.0 & 12.6 & 12.5 & 12.5 & 11.7 & 12.2 \\
\hline 06-624 & 1.05 & 10.3 & 12.7 & 13.8 & 12.3 & 11.8 & 12.6 \\
\hline 06-625 & 1.01 & 10.1 & 8.6 & 8.4 & 12.8 & 12.5 & 12.1 \\
\hline 06-800 & 0.89 & 13.9 & 17.5 & 23.6 & 12.5 & 13.4 & 13.1 \\
\hline 06-800 & 0.93 & 13.6 & 14.9 & 25.6 & 12.6 & 12.7 & 12.5 \\
\hline 06-906 & 1.20 & 13.1 & 14.5 & 20.5 & 11.7 & 12.3 & 11.7 \\
\hline 06-906 & 0.92 & 15.5 & 14.3 & 19.0 & 11.2 & 12.4 & 11.8 \\
\hline 06-906 & 0.65 & 18.4 & 18.0 & 33.9 & 10.5 & 12.2 & 12.8 \\
\hline 06-906 & 1.08 & 13.4 & 15.6 & 19.4 & 11.7 & 11.7 & 12.0 \\
\hline 06-914 & 1.11 & 11.0 & 11.6 & 21.4 & 11.7 & 12.2 & 12.0 \\
\hline 06-914 & 0.79 & 14.4 & 16.0 & 25.3 & 12.4 & 13.0 & 12.1 \\
\hline 06-999387 & 1.04 & 11.3 & 13.6 & 11.4 & 12.1 & 12.3 & 11.9 \\
\hline 06-СР070 & 0.07 & 19.0 & 18.3 & 27.4 & 11.9 & 24.2 & 18.9 \\
\hline 06-СР160 & 0.11 & 29.6 & 29.8 & 36.9 & 13.7 & 5.2 & 26.8 \\
\hline 06-СР160 & 0.22 & 23.1 & 28.2 & 34.1 & 9.7 & 13.3 & 25.5 \\
\hline 06-СР160 & -0.06 & $\infty$ & $\infty$ & $\infty$ & $\infty$ & $\infty$ & $\infty$ \\
\hline 06-СР161 & 0.47 & 17.7 & 28.9 & 39.4 & 10.7 & 16.9 & 15.4 \\
\hline 06-СР162 & 0.33 & 18.5 & 23.1 & 39.3 & 10.5 & 16.4 & 12.2 \\
\hline 06-СР215 & 1.24 & 13.7 & 15.5 & 19.5 & 10.6 & 11.8 & 11.2 \\
\hline $12-45$ & 1.51 & 11.3 & 8.4 & 24.4 & 10.1 & 13.2 & 10.1 \\
\hline $12-868$ & 1.31 & 12.6 & 13.5 & 15.0 & 11.0 & 11.8 & 11.5 \\
\hline 12-U12V AC & 1.50 & 10.7 & 13.5 & 21.9 & 11.5 & 11.5 & 12.3 \\
\hline 23-113 & 0.15 & 31.7 & 16.2 & 56.0 & 12.6 & 14.3 & 14.2 \\
\hline 23-113 & 0.77 & 17.8 & 20.2 & 46.0 & 11.8 & 11.5 & 12.6 \\
\hline 23-128 & 0.09 & 17.9 & 19.4 & 26.1 & 11.0 & 19.8 & 13.7 \\
\hline $23-128$ & 0.39 & 15.6 & 16.6 & 22.6 & 12.4 & 12.8 & 18.2 \\
\hline 23-128 & 0.02 & 27.6 & 29.8 & 34.5 & 14.0 & 16.6 & 16.5 \\
\hline $23-128$ & 0.73 & 16.9 & 17.9 & 20.9 & 12.6 & 14.0 & 13.4 \\
\hline
\end{tabular}




\begin{tabular}{|c|c|c|c|c|c|c|c|}
\hline 23-151 & 0.91 & 15.7 & 14.6 & 22.6 & 12.7 & 13.4 & 13.5 \\
\hline 23-151 & 0.48 & 21.4 & 24.6 & 28.8 & 12.9 & 14.5 & 13.3 \\
\hline 23-151 & 0.64 & 14.8 & 14.2 & 19.6 & 11.7 & 12.6 & 12.8 \\
\hline 23-151 & 0.65 & 14.2 & 12.6 & 12.1 & 12.8 & 12.2 & 13.5 \\
\hline $23-154$ & 0.98 & 13.2 & 13.6 & 21.3 & 12.0 & 13.5 & 12.8 \\
\hline 23-158 & 0.75 & 16.0 & 21.3 & 21.2 & 12.6 & 15.4 & 25.8 \\
\hline 23-190 & 1.03 & 20.8 & 23.0 & 27.5 & 12.0 & 13.0 & 15.8 \\
\hline 23-190 & 1.28 & 16.9 & 22.6 & 23.6 & 12.0 & 13.4 & 13.6 \\
\hline 23-190 & 0.11 & 31.5 & 33.0 & 31.1 & 21.0 & 34.0 & 25.7 \\
\hline 23-190 & 0.09 & 31.6 & 37.0 & 36.6 & 20.9 & 34.1 & 25.5 \\
\hline 23-610 & 0.17 & 19.2 & 19.4 & 25.5 & 14.1 & 15.3 & 14.9 \\
\hline 23-703 & 0.23 & 17.6 & 17.8 & 28.4 & 10.4 & 15.3 & 13.6 \\
\hline 23-703 & 0.41 & 17.4 & 17.7 & 23.1 & 12.1 & 14.5 & 13.4 \\
\hline 23-703 & 0.32 & 19.7 & 18.4 & 37.8 & 10.6 & 13.8 & 15.8 \\
\hline 23-703 & -0.25 & $\infty$ & $\infty$ & $\infty$ & $\infty$ & $\infty$ & $\infty$ \\
\hline 24-A03 & 0.49 & 17.2 & 16.9 & 21.9 & 12.9 & 12.4 & 14.7 \\
\hline $25-202674$ & 1.30 & 11.8 & 13.7 & 15.0 & 11.8 & 12.4 & 10.6 \\
\hline 26-2204 & 1.06 & 17.1 & 14.4 & 17.1 & 12.6 & 11.5 & 11.8 \\
\hline 27-5110 & 0.61 & 17.3 & 16.4 & 26.7 & 12.7 & 13.6 & 12.0 \\
\hline 27-5110 & 0.57 & 16.3 & 16.2 & 21.1 & 11.8 & 13.2 & 12.0 \\
\hline 35-2215 & 0.47 & 17.6 & 22.2 & 24.7 & 12.3 & 12.4 & 16.0 \\
\hline
\end{tabular}

The final table in this appendix shows the log metal ratios from wipe samples obtained in January 2009 in which the Be concentration exceeds the DOE RC. The coding is the same as in the previous table.

There are a few wipe samples in which the Y/Be ratio is lower than the normal range for NTS soils. Those with the ratio mildly lower than the normal range were obtained in 06-800 and 24-703; in one of these (in 06-800) the $\mathrm{Nb} / \mathrm{Be}$ ratio was also rather low. That wipe was taken on a lathe. Otherwise, the $\mathrm{Cu} / \mathrm{Be}$ ratio seems somewhat high for a number of these samples and the $\mathrm{Ni}$ /Be ratio seems somewhat low; given the wide range of variation of these ratios seen in the NTS soils, less importance is placed on these ratios.

\begin{tabular}{|c|c|c|c|c|c|c|}
\hline \multicolumn{7}{|c|}{ Ghost Wipe (January 2009) Metal Ratio Results } \\
\hline \multirow[b]{3}{*}{ Facility } & $\mathrm{Co} / \mathrm{Be}$ & $\mathrm{Ni} / \mathrm{Be}$ & $\mathrm{Cu} / \mathrm{Be}$ & Y/Be & $\mathrm{Nb} / \mathrm{Be}$ & $\mathbf{U} / \mathbf{B e}$ \\
\hline & \multicolumn{6}{|c|}{ Coded values for $\log ($ ratio $)$} \\
\hline & \multicolumn{6}{|c|}{ Soil median $=12$; one unit $=25 \%$ change } \\
\hline Soil 2.5 percentile & 5.7 & 5.1 & 3.0 & 10.0 & 9.7 & 8.4 \\
\hline Soil Median & 12.0 & 12.0 & 12.0 & 12.0 & 12.0 & 12.0 \\
\hline Soil 97.5 percentile & 18.3 & 18.9 & 21.0 & 14.0 & 14.3 & 15.6 \\
\hline $01-201959$ & 20.0 & 23.0 & 28.6 & 13.4 & 16.9 & 13.9 \\
\hline 01-202479 & 11.9 & 12.9 & 8.0 & 13.5 & 12.9 & 12.6 \\
\hline 01-202479 & 11.6 & 14.8 & 15.7 & 12.4 & 12.2 & 11.0 \\
\hline 01-202479 & 10.7 & -4.8 & -1.0 & 12.9 & 13.7 & 12.1 \\
\hline 03-3СЗ6 & 10.7 & -8.3 & 4.5 & 11.4 & 13.7 & 11.1 \\
\hline 03-3С36 & 11.6 & $-\infty$ & 14.3 & 11.5 & 13.4 & 11.9 \\
\hline $06-624$ & 14.0 & 13.1 & 25.9 & 12.6 & 12.6 & 11.5 \\
\hline 06-624 & 12.9 & 7.7 & 18.6 & 12.7 & 14.0 & 12.4 \\
\hline $06-624$ & 12.8 & 9.5 & 23.7 & 12.6 & 13.7 & 12.4 \\
\hline $06-800$ & 22.0 & 5.2 & 21.4 & 12.4 & 13.7 & 12.5 \\
\hline 06-800 & 14.4 & 10.9 & 23.3 & 12.4 & 14.5 & 13.0 \\
\hline 06-800 & 13.4 & 11.8 & 21.8 & 11.5 & 12.5 & 12.3 \\
\hline 06-800 & 16.2 & 24.3 & 26.0 & 12.1 & 13.6 & 12.8 \\
\hline 06-800 & 17.0 & 15.2 & 31.8 & 12.1 & 13.1 & 12.8 \\
\hline
\end{tabular}




\begin{tabular}{|c|c|c|c|c|c|c|}
\hline 06-800 & 17.6 & 15.8 & 34.8 & 11.6 & 12.7 & 12.5 \\
\hline 06-800 & 13.0 & $-\infty$ & 21.3 & 12.1 & 12.7 & 12.1 \\
\hline 06-800 & 13.7 & 13.7 & 28.5 & 9.8 & 11.0 & 10.9 \\
\hline 06-800 & 14.8 & 14.9 & 23.6 & 11.3 & 12.5 & 13.1 \\
\hline 06-800 & 14.9 & 11.4 & 24.0 & 12.2 & 12.7 & 12.6 \\
\hline 06-800 & 19.7 & 20.6 & 21.2 & 12.5 & 13.6 & 12.4 \\
\hline 06-800 & 16.5 & 19.6 & 27.9 & 12.8 & 13.8 & 12.8 \\
\hline 06-800 & 15.6 & 19.5 & 25.9 & 12.3 & 13.4 & 16.9 \\
\hline 06-800 & 20.8 & 23.1 & 37.6 & 12.0 & 13.3 & 12.3 \\
\hline 06-800 & 17.4 & 40.8 & 58.3 & 8.1 & 1.2 & 8.7 \\
\hline 06-906 & 17.3 & 18.9 & 22.1 & 11.9 & 13.6 & 12.8 \\
\hline 06-906 & 15.8 & -1.8 & 22.2 & 12.2 & 12.8 & 13.4 \\
\hline 06-906 & 16.0 & 13.7 & 17.9 & 12.3 & 13.8 & 12.6 \\
\hline 06-906 & 21.2 & $-\infty$ & 33.5 & 12.0 & 13.0 & 13.8 \\
\hline 06-914 & 18.2 & 19.5 & 31.9 & 12.4 & 13.0 & 12.7 \\
\hline 06-914 & 23.5 & 20.3 & 32.1 & 12.6 & 13.3 & 13.2 \\
\hline 06-914 & 20.8 & 13.4 & 29.6 & 12.1 & 13.0 & 15.4 \\
\hline 06-914 & 16.4 & 15.3 & 30.3 & 12.3 & 12.9 & 13.7 \\
\hline 06-914 & 19.8 & 23.0 & 30.1 & 12.2 & 13.4 & 13.7 \\
\hline 06-999387 & 10.4 & 5.0 & 11.6 & 12.3 & 13.3 & 12.3 \\
\hline 06-999387 & 10.7 & 7.0 & 10.7 & 12.3 & 13.2 & 12.3 \\
\hline 06-999387 & 11.1 & $-\infty$ & 15.2 & 12.3 & 13.2 & 12.2 \\
\hline 06-СР-070 & 15.2 & 14.5 & 26.2 & 13.9 & 14.5 & 15.4 \\
\hline 06-СР-070 & 12.9 & 15.1 & 24.9 & 10.7 & 11.6 & 15.2 \\
\hline 06-СР-160 & 15.0 & 15.0 & 26.8 & 12.1 & 12.8 & 12.2 \\
\hline 06-СР-162 & 18.6 & 11.2 & 17.4 & 12.2 & 13.5 & 13.4 \\
\hline 06-СР-162 & 24.7 & 20.5 & 31.9 & 12.5 & 12.8 & 12.5 \\
\hline 06-СР-215 & 20.1 & 12.3 & 28.6 & 11.4 & 12.0 & 13.9 \\
\hline 12-U12 & 9.5 & 2.1 & 21.9 & 11.3 & 13.5 & 11.6 \\
\hline 23-151 & 17.0 & 13.1 & 18.2 & 14.4 & 14.3 & 14.9 \\
\hline 23-190 & 15.6 & 25.1 & 25.9 & 12.7 & 13.3 & 16.2 \\
\hline 23-190 & 20.6 & 23.7 & 28.0 & 12.2 & 14.1 & 15.8 \\
\hline 23-610 & 11.9 & $-\infty$ & 8.4 & 12.0 & 13.1 & 11.9 \\
\hline 23-703 & 14.3 & 18.2 & 33.2 & 8.1 & 11.4 & 12.4 \\
\hline 25-202616 & 13.4 & 15.2 & 10.2 & 12.0 & 13.3 & 12.0 \\
\hline
\end{tabular}




\section{Appendix 3 \\ CHEMICAL ANALYSIS AND DATA ISSUES}

\section{Overview}

Most of the first 5,000 or so samples obtained for this study were analyzed by DataChem, an independent, subcontract laboratory accredited by the American Industrial Hygiene Association (AIHA). With the exception of minor issues that are addressed by subtracting the means of appropriate sets of blank observations, these data are used as provided by the laboratory. The minor issues are discussed in Appendix 4. These data are designated "DC" in the discussions and plots that follow. The wipes were taken and analyses performed from late 2002 through the middle of 2003.

The large majority of the next 6,000 or so samples (obtained from the middle of 2003 into 2004) were analyzed by Bechtel Nevada (BN) personnel on an inductively coupled plasma mass spectroscopy (ICP-AES) instrument acquired for that purpose, using the 234.861-nanometer (nm) emission line. As a precaution, side-by-side wipes were obtained for every tenth sample obtained at most facilities, in addition to the usual quality control (QC) samples. One of each pair was analyzed using this system (BN-AES), and the other by DC. For logistical reasons unrelated to the study itself, review of the QC sample results was delayed. Nonetheless, analyses proceeded using BN-AES (except for the DC side-by-side samples) with the 234.861-nm line.

When the QC side-by-side data were eventually evaluated, significant discrepancies between the BN-AES and DC data were found. The discrepancies appeared greater in sample pairs obtained in shop and similar facilities than those obtained in office buildings and similar facilities. Considerable effort was expended in determining the cause of these discrepancies, as is discussed in "What is a Beryllium Measurement? A Critical Look at Beryllium Quantitation" (C. B. Davis, D. E. Field, J. W. Hess, and D. A. Jensen, presented the Second Symposium on Beryllium Particulates and Their Detection, November 8-9, 2005, Salt Lake City, UT). It was determined that the source of the discrepancies was interference due to a minor iron (Fe) peak located at approximately $234.841 \mathrm{~nm}$, just outside the window used by the ICP-AES instrument in its beryllium (Be) analyses. This Fe interference was unanticipated - this peak is not listed in the NIST Handbook of Basic Atomic Spectroscopic Data, for example - but is nonetheless sufficiently large to significantly decrease the instrument's response to low concentrations of Be using the 234.861-nm spectral line. One of the clues to the nature of the interference is the types of facilities in which the discrepancies were greatest.

Upon determination of the nature of the problem, a study was conducted to identify a solution. It turns out that for many of the samples analyzed by BN-AES a simple correction based on the iron peak height (FePH) can be made, so long as the FePH is not too great, although the resulting adjusted data will be somewhat more variable than would otherwise be anticipated. Upon comparing the resulting $\mathrm{BN}$-adj data with the $\mathrm{DC}$ results from the side-by-side wipes, a modest correlation (0.547 after deleting a small number of outliers) is found. This is not unreasonable given the generally low Be values found in the relatively "clean" samples which also have low Fe. Also, one must recognize that side-by-side samples are not true duplicates, particularly when sampling particulate materials. The second major section of this appendix details the FePH correction study. 
The remaining BN-AES samples (approximately 900 in number) were reanalyzed using a newly acquired ICP-MS instrument (denoted "BN-MS" or "MS_arch"). These reanalyses are somewhat problematic, in that they use archived digestates from the original analyses that had been stored for several years beyond the usual holding time limit. Again, the best comparison for evaluating these samples is the side-by-side DC analyses. In this case, the side-by-side evaluations are not limited to relatively "clean" samples, and (again, after deleting a few outliers) a decent correlation of 0.876 is seen. The BN-MS data do tend to be higher than the DC data, though; a weighted-least-squares line is DC $=0.5646 *$ MS_arch. On the other hand, BN-MS analyses of archived $\mathrm{Fe} / \mathrm{Be}$ spiked samples discussed in the second section suggest that the BN-MS analyses do not tend to be either systematically high or low compared with the true values. Those $\mathrm{Fe} / \mathrm{Be}$ spiked samples (prepared in October 2005) have not been held for nearly as long as the archived digestates of environmental samples, which had been held since late 2003 through 2004.

These results present a quandary. Clearly the BN-MS results are informative, given the high correlation with DC side-by-side data. Should these (a) be adjusted by the DC $=0.5646 *$ MS fitted line to produce data more nearly resembling those obtained from the third-party AIHIaccredited lab, (b) be used as is, or (c) treated in some intermediate fashion? After discussion and deliberation, it was decided to follow the conservative approach of relying on the MS_arch data as is. The third major section of this appendix presents detailed analyses of these data.

\section{Adjusting ICP-AES (234.861 nm) Measurements for Iron Peak Interference}

The nominal Be wavelength used for the BN- - - AES analyses was $234.861 \mathrm{~nm}$, with a typical spectral window extending $\pm 0.016 \mathrm{~nm}$. A broad interference peak is found centered at approximately $234.841 \mathrm{~nm}$. This peak is found in virtually all analyses, suggesting that the interferent is present in all samples, even blanks. For example, the figure to the right shows a spectrum obtained from analysis of a ghost wipe spiked only with 0.01 micrograms $(\mu \mathrm{g}) \mathrm{Be}$. The peak in the center is the Be peak; that on the left is the interferent peak. For comparison with subsequent discussions, the interferent peak height is 1,739 in this spectrum.

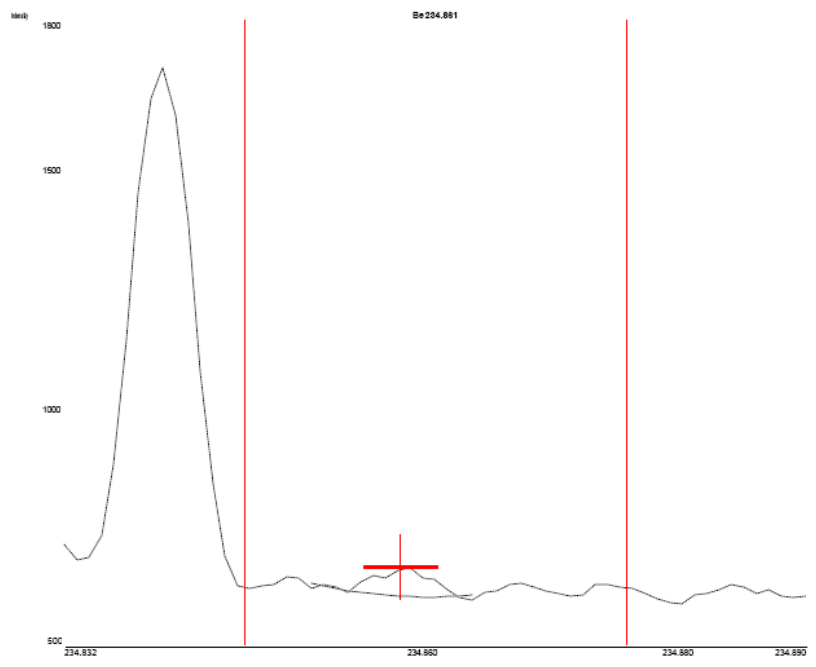

Analyses of ghost wipe samples spiked with a variety of substances suggested that the interferent is virtually certainly Fe. Therefore, a quantitative model of the effect of the Fe peak on Be quantitation was developed, toward a goal of providing a defensible adjustment procedure for samples in which the FePH is not excessive. Since the release criterion (RC) for Be wipe samples is $0.2 \mu \mathrm{g}$ per wipe, Be concentrations of interest range from 0.0 to $0.2 \mu \mathrm{g} / \mathrm{sample}$.

Analyses of spiked ghost wipes were conducted by BN personnel on October 12, 13, and 27, 2005. Conclusions obtained from analyzing the resulting data are the following: 
- The upper limit of FePH values for making adjustments is approximately 65,000. (FePH values as high as 12,000,000 have been found.)

- For measured Be values less than approximately $0.2 \mu \mathrm{g}$, a reasonable adjustment is $\mathrm{BN}$-adj $=\frac{\mathrm{M}+0.20626 \mathrm{~F}}{1-4.1405 \mathrm{~F}}$, where $\mathrm{BN}$-adj is the adjusted value, $\mathrm{M}$ the reported value, and $\mathrm{F}=\mathrm{FePH} \times 10^{-6}$. An M above $0.2 \mu \mathrm{g}$ already exceeds the RC; since the adjustment can only increase that value, there is no reason to adjust measured values above $0.2 \mu \mathrm{g}$.

- This adjustment should provide BN-adj measurements that are approximately unbiased for $0 \leq \mathrm{FePH} \leq 65,000$ and $0 \leq \mathrm{T} \leq 0.2$, where $\mathrm{T}$ is the true concentration (i.e., the amount of Be spiked into the ghost wipe). BN-adj measurements will be somewhat "noisier" than unadjusted measurements, by a proportion ranging from about $19 \%$ at $\mathrm{T}=0$ to about $27 \%$ at $\mathrm{T}=0.2 \mu \mathrm{g}$.

- The increased analytical variability due to the adjustment will be only a small proportion of the total variability where there is a substantial spatial component of variation. The bottom-line statistical procedure automatically accounts for the inherent variability of measurement data. Hence, there is no reason to modify the bottom-line statistical procedure (upper tolerance limit or prediction limit) because of the adjustment.

Details

Ghost wipes were spiked with solutions of Be and Fe and analyzed using the BNAES instrument on three days (October 12, 13, and 27, 2005, referred to as Days 1, 2, and 3). Analysis of the Day 1 and Day 2 data established the soundness of the concept of making this sort of adjustment, and established that the upper limit of FePH should be in the

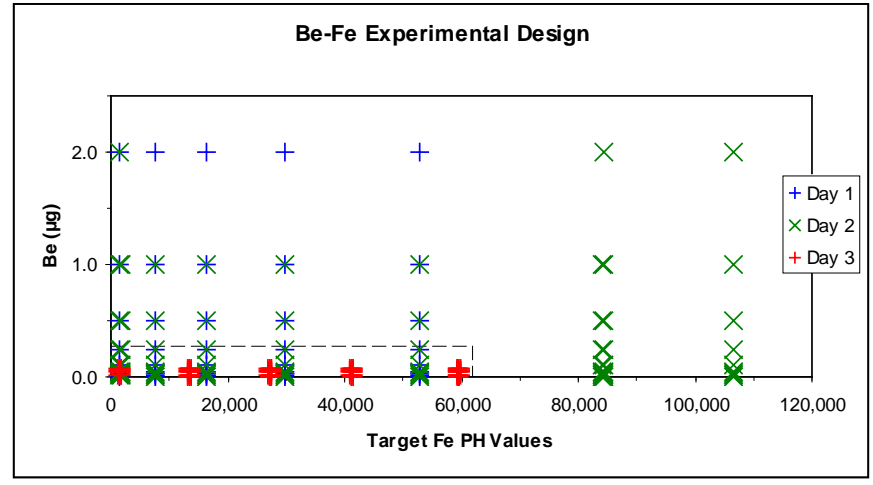
neighborhood of 70,000. Adding the Day 3 data further confirmed the concept and provided additional insight into the nature of the measurement variation involved. A preliminary study with Fe alone had allowed the specification of spiking concentrations that achieved approximately the target FePH values. The design is represented in the accompanying figures; the second is a blown-up version of the lower left portion of the first.

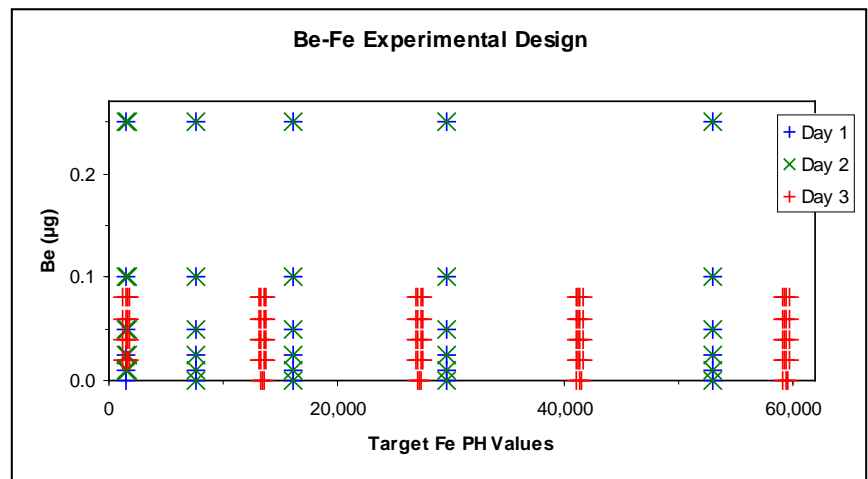
Replicates are represented by slightly “jittered” points, particularly for Day 3.

A series of straight-line fits of measured value $\mathrm{M}$ as a function of FePH were made, one for each separate value of the true concentration $(\mathrm{T})$. In each case the intercept of the line was not significantly different from $\mathrm{T}$, so the fitted line was forced through the point $(0, \mathrm{~T})$. Since there is a negative slope in every case, and since ghost wipes apparently do contain some Fe, this implies 
that the fitted $\mathrm{M}$ value will be slightly less than $\mathrm{T}$ even if no Fe is added to the wipe. The slopes themselves form a nice linear trend as a function of $\mathrm{T}$, so a second level of fitting (of slope to $\mathrm{T}$ ) was employed. This doubly fitted model is $\mathrm{M}=\mathrm{T}+(\mathrm{a}+\mathrm{bT})^{*} \mathrm{~F}+$ error, where $\mathrm{F}=\mathrm{FePHx} 10^{-6}$ for convenience. The fitted values are $a=-0.20626$ and $b=-4.1405$. If we insert an actual measurement value for $\mathrm{M}$, set “error" to 0 , and solve for $\mathrm{T}$, the result is the adjusted measurement BN-adj $=\frac{M+0.20626 \mathrm{~F}}{1-4.1405 \mathrm{~F}}$.

The following plots show all measured and adjusted values in the region of interest.
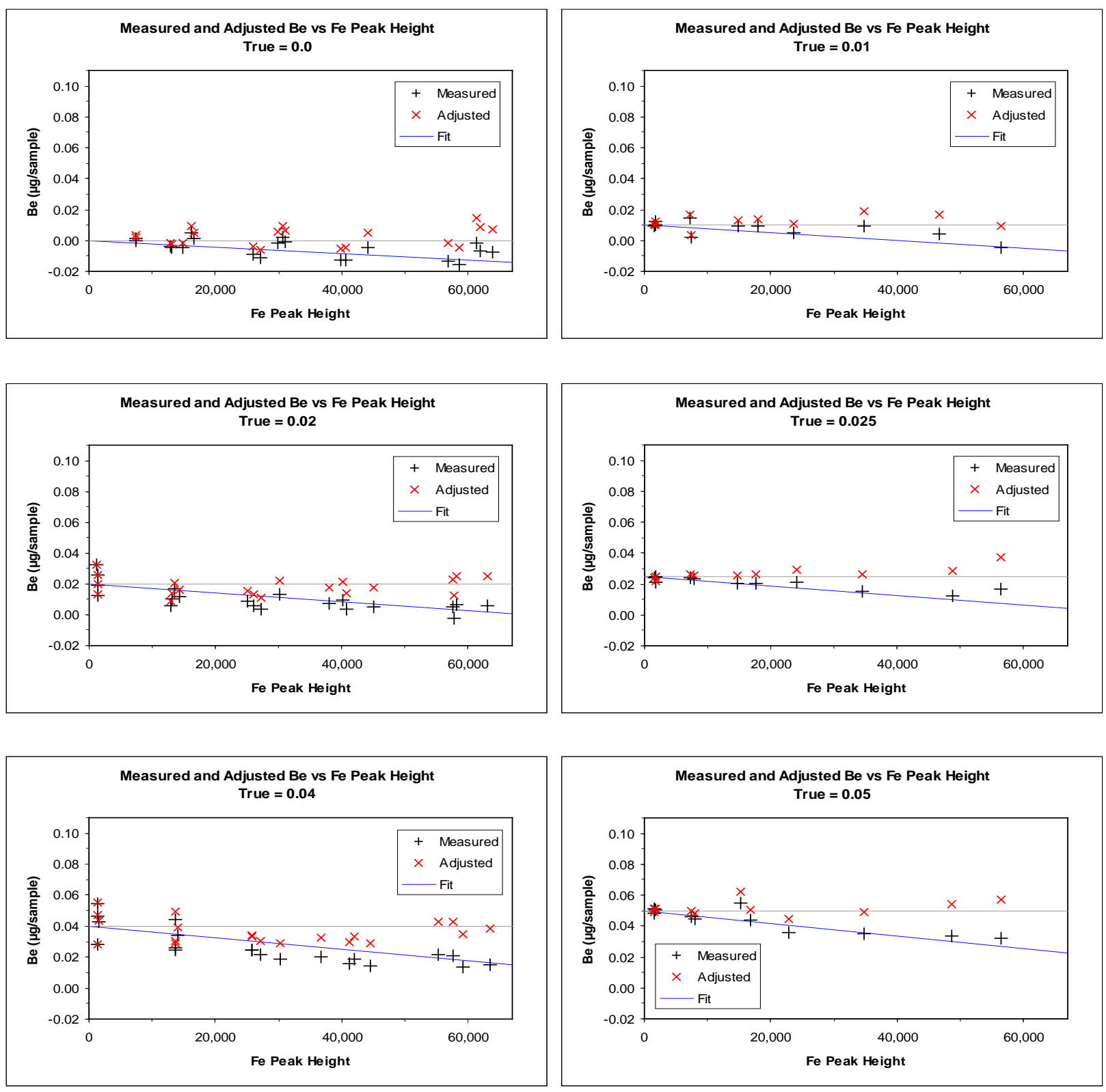

Worker Environment Beryllium Characterization Study 4 Appendix 3 

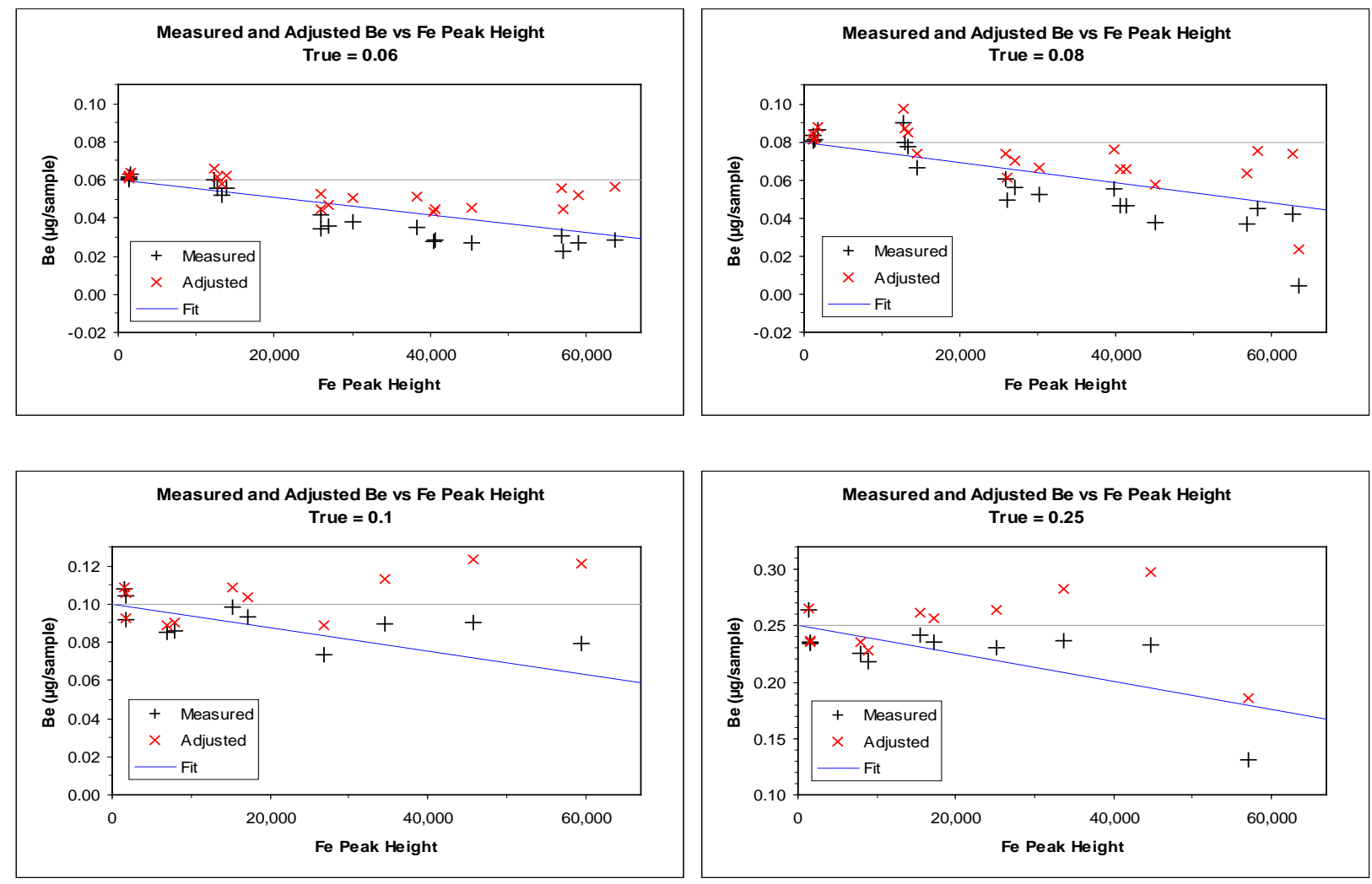

Overall the fitted values estimate their targets nicely, as expected. There are minor deviations from day to day, as seen in the plot at right; these will be of little practical significance.

The standard deviations of the adjusted values are shown in the next plot, along with a fitted curve (blue) of form $\mathrm{SD}^{2}=\mathrm{A}^{2}+\mathrm{B}^{2} \mathrm{~T}^{2}$. This model provides for two statistically independent components of analytical variability: one (with standard deviation $\mathrm{A} \approx$ 0.0056) independent of $T$, and the other with relative standard deviation $\mathrm{B}(\approx 0.064)$. The green curve is fitted to standard deviations of ghost wipes spiked with Be only; the distance between the curves reflects the noise added by the FePH adjustment process.
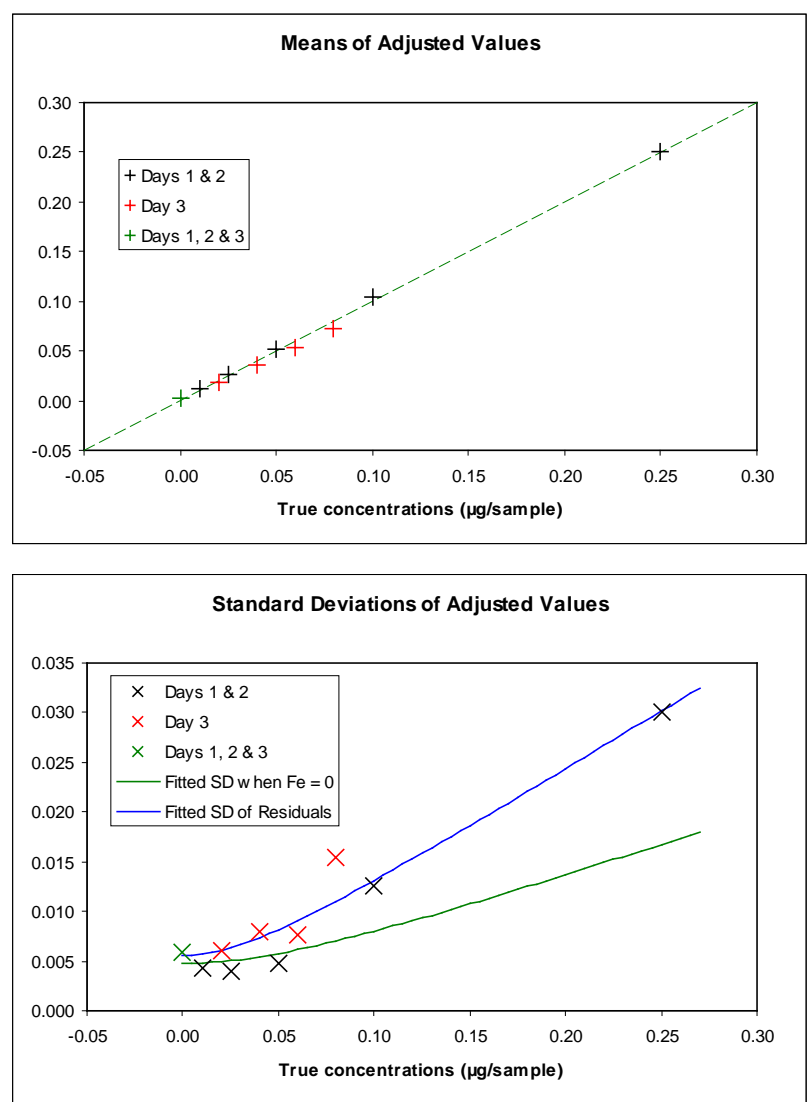


\section{$\underline{\text { Validation with Side-by-Side DC Samples }}$}

$\mathrm{BN}$-adj values were computed for all BN-AES analyses with $\mathrm{FePH}<65,000$. As discussed in Appendix 4, the mean of field blanks for the entire study period was slightly positive (0.0062), so data were blank-corrected by subtracting this quantity. (A similar blank correction was made for the DC and BN-MS data as well.) The following series of plots show the side-by-side BNMS and DC blank-corrected (_BC) values. The area included in dotted lines in the first is the whole of the second, and similarly with the second and third.
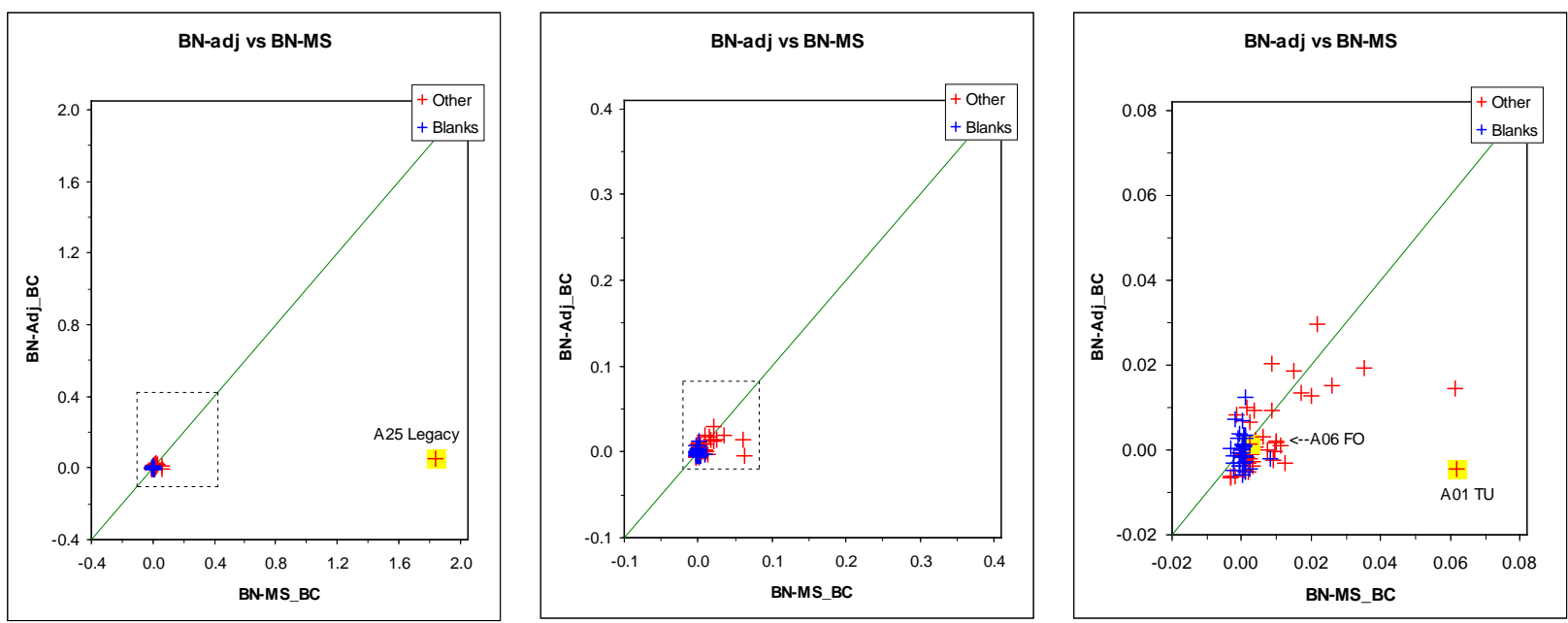

Three outliers are highlighted in yellow, identified in at least two of the three two-way comparisons of paired values; these are omitted from the weighted-least-squares analysis of the BN-MS and DC pair data in the following section. The causes of these outliers are not known. Otherwise, as stated, the correlation between BN-adj and DC is modest, but that is due mostly to the fact that nearly all Be values are low. This is, of course, a consequence of the exclusion of samples with $\mathrm{FePH}>65,000$.

\section{Reanalysis of Archived Samples by ICP-MS}

Around 900 of the digested wipes from the BN-AES analyses were reanalyzed (December 2006-June 2007) using an ICP-MS instrument. These include the majority of those samples with $\mathrm{FePH}>65,000$, where those samples are critical to the data analysis for their particular facilities, along with a number of QC samples for which BN-adj and/or DC data values are available. In this section the side-by-side BN-MS (MS_arch) and DC pair and the BN-MS and BN-adj pair of data are reviewed.

The side-by-side comparisons with DC data are not constrained by the limited range since Fe is not involved. There are 113 pairs of samples, of which 8 are identified as outliers. With the remaining 105 pairs the correlation between the BN-MS and DC values is a respectable 0.876 , as stated previously.

There is concern, however, in that the slope of the linear relationship between the DC and BNMS data is not 1 , as desired, but rather a little less than 0.6; a weighted-least-squares fit gives a 
slope of 0.56458 . (The intercept was not significantly different from zero, after blank correction, and accordingly was omitted from the predictive model.) The " $y=x$ " line is green in the following plots, whereas the weighted least square (WLS) fitted line for predicting the DC values from the MS values is in blue.
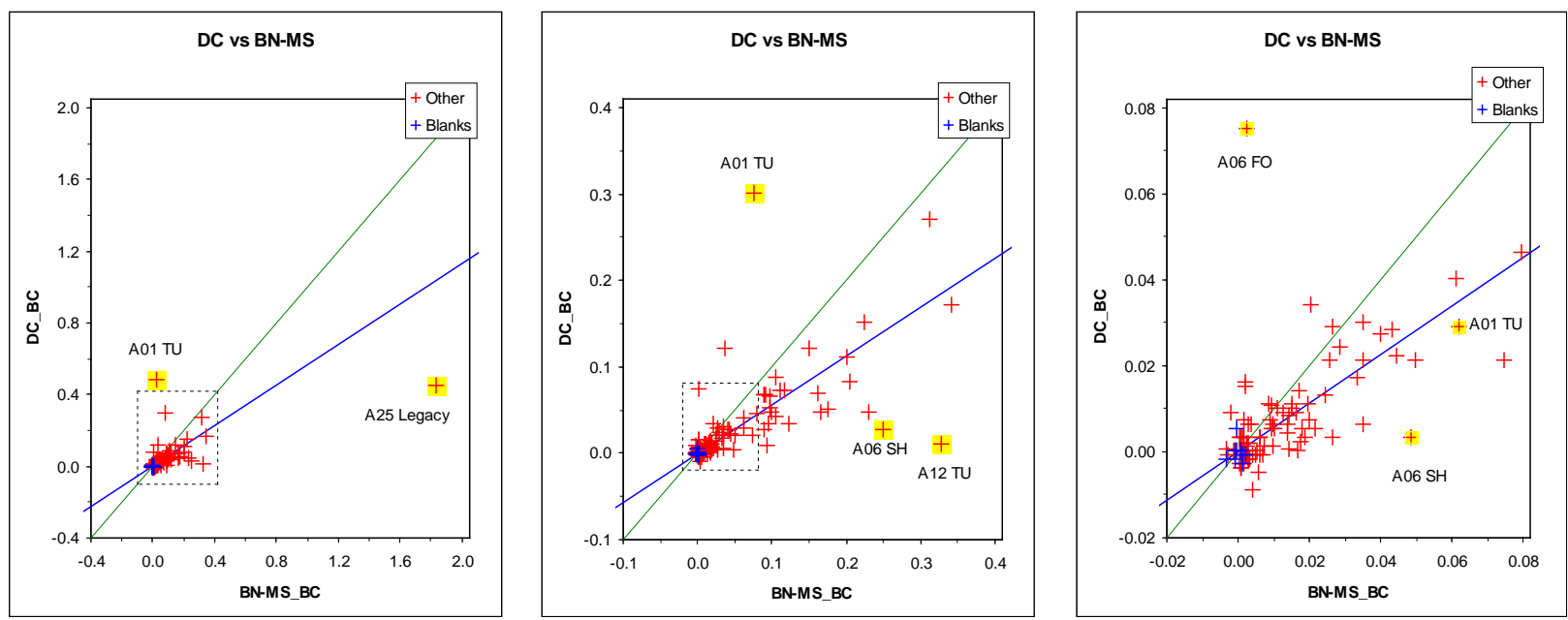

Outliers identified in these plots, and omitted in the WLS fit, include those identified in the plots presented in the previous section. Since these are side-by-side wipes, rather than true duplicates, occasional outliers can be due to one sample of a pair including a particle not included in the other, so these are not unexpected.

For comparison, plots of BN-adj vs. BN-MS results are included as well. With the outliers omitted, the correlation is a moderate 0.617, again related to the compressed range of available data once the samples with higher FePH values are removed. (With the right-most non-highlighted point in the right plot omitted, the correlation rises to only 0.669.) These data are not inconsistent with the WLS fit obtained from the DC vs. BN-MS plot, although the small range of values allows more variability in the slope of a fitted line.
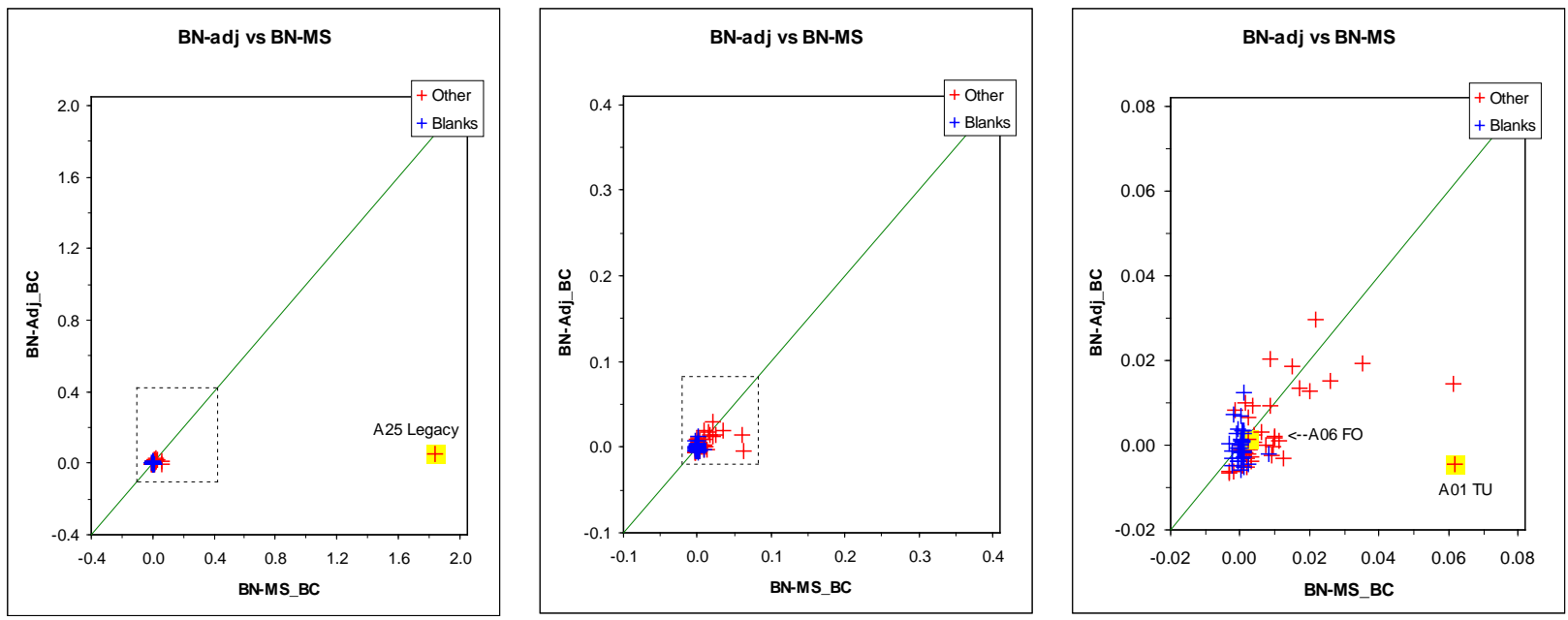
As mentioned, reanalysis of a few archived digestates from the Fe/Be spiked ghost wipe study suggests that there might be no similar disparity between the BN-MS analyses of archived samples and the actual concentrations. A number of explanations are possible. One is that digestates from the Fe/Be study have not been in storage nearly as long as the digestates of environmental samples, and have not experienced concentration due to aging. Another explanation, though, is that the DC analyses (performed using ICP-AES, but with a different spectral line) may also be subject to interferences other than Fe. There may be other explanations; it is not possible to decide among the various possibilities at this point.

\section{$\underline{\text { Path Forward }}$}

The BN-adj data are used without concern, as stated previously.

The BN-MS (MS_arch) reanalysis data are also used as is. This analysis is conservative, because the slope of the WLS line discussed above is considerably less than 1 . If a facility satisfies the established criteria using this approach, no further analysis is performed.

If a facility does not satisfy the RC using this conservative approach, further investigation is needed. This further investigation involves resampling facilities as needed, at least in the vicinity of high values, possibly preceded by a preemptive cleaning. The fresh samples, obtained during January 2009 (February 2008 for 06-908) were again analyzed by ICP-MS; these data are designated MS_new. In addition, bulk samples were obtained inside the facility as well is in the soils in the vicinities of the facilities, as described in the following section.

\section{Alternate Facility Evaluation Methods}

The discussion and methods discussed so far in this report deal with satisfying regulatory criteria framed in terms of the Title 10 Code of Federal Regulations 850 U.S. Department of Energy $\left(0.2 \mu \mathrm{g} / 100 \mathrm{~cm}^{2}\right)$ [0.2 micrograms/100 square centimeters]. There is an alternative also contained in that regulation that can be considered, which is to show that Be concentrations in bulk samples obtained within a facility are not higher than those in surrounding soils. The apparent objective of that regulatory alternative is to demonstrate that the Be contained in dust inside the facility is not anthropogenic, but rather could reasonably have originated in native soils.

Another way of approaching this issue would be to compare ratios of certain metals contained in either bulk or wipe samples obtained within the facility with those found in native soils. Both of these approaches were used with certain facilities. A more detailed discussion of these approaches is found in Appendix 4. 


\section{Appendix 4 ADDITIONAL STATISTICAL ISSUES}

\section{Overview}

This appendix discusses several technical statistical issues.

The conventional assumption is that environmental contaminant concentrations follow lognormal models; that is, that logs of the concentrations have normal distributions. The distributions of measurements, however, are more complicated; most obviously, measurements can be negative, which is inconsistent with the lognormal assumption. Nearly all measurements used in this study are uncensored, meaning that actual ("raw") values are reported and used, in order to avoid the loss of information (statistical power) inherent in censoring data. The main issue discussed in this appendix is the treatment of these uncensored data.

Also, recall that the regulation that provides the U.S. Department of Energy (DOE) regulatory criteria (RC) allows an alternate comparison, which is that surface beryllium (Be) concentrations on items to be released to the public should not be higher than concentrations in neighboring soils. The intent is apparently to allow one to distinguish between naturally occurring and anthropogenic Be. An extension of that concept is to evaluate not only Be concentrations in bulk material samples but also ratios of other common metals with Be, a so-called "fingerprint" analysis. The other metals involved are cobalt (Co), nickel (Ni), copper (Cu), yttrium (Y), niobium $(\mathrm{Nb})$, and uranium-238 $\left({ }^{238} \mathrm{U}\right)$; these analyses are available in wipe and bulk samples analyzed using the inductively coupled plasma mass spectroscopy (ICP-MS) instrument. This appendix also includes evaluations of the soil Be concentrations and metal ratios and a discussion of the comparison of bulk and wipe samples with the soil data.

The structure of this appendix is as follows:

- The main issue and its implications are first discussed in greater detail.

- The distributions of blank measurements are evaluated. It turns out that there are highly statistically significant and non-negligible differences among groups of blank measurements. To compensate for these differences, data are "blank-corrected"; that is, adjusted for the apparent low-end bias as reflected in the blank measurements.

- A simple heuristic for handling the negative values is described. That heuristic involves adding a small constant (delta) to all data values, doing the lognormal analysis, and then subtracting delta from the resulting prediction level (PLall) or upper tolerance limit (UTL). The algorithm for selecting delta is presented; delta varies by analytical method and type of facility.

- A method for performing UTL analyses using all Individual Work Area (IWA) data, rather than just the IWA maxima, is presented. This is termed "UTL cluster analysis"; 
the observations from a given IWA are treated as coming from a cluster in statistical survey terms, where one allows for the possibility that values in the same cluster might be positively correlated.

- Finally, an analysis of Be data and metal ratio data in soil samples is presented.

\section{The Main Statistical Issue}

As discussed in Appendix 1, the lognormal statistical model is used as the basis for the statistical analyses presented in this document. There is a considerable weight of tradition behind the selection of this model for distributions of environmental contaminants. A mechanism that might generate this model is the following: if one considers the concentration at a given point to be a random fraction of a random fraction of ... of some source, that concentration would be a product of random quantities, and so one might posit a central-limit-theorem argument on the log scale to support the lognormal assumption. On the other hand, the common use of the lognormal model is likely based much more on perceived ease of use, in that the model is non-negative and right-skewed, and one can "just take logs and use normal-theory methods.”

This model is somewhat simplistic, but nonetheless useful if handled with care. In the words of the eminent statistician G. E. P. Box, "All models are wrong, but some are useful." ("Robustness in the Strategy of Scientific Model-Building”, in R. Launer and G. Wilkenson, eds, Robustness in Statistics, 1979). Recently other not-so-heavy-tailed models have been proposed for environmental contaminants, notably gamma distribution models. Empirical evidence supporting one model over the other is difficult to come by, since the main differences are in the extreme upper tails where data are rare. The not-so-heavy-tailed models are likely to give more palatable upper limits, though, and perhaps that is a reason for their appeal; one may therefore consider the lognormal models to be possibly conservative.

Although the lognormal model may arguably be appropriate for the distribution of Be concentrations, sample preparation and analytical variation ("error") impact, in particular, the lower tail of the distribution of measurements. For some facilities over half the measurements are negative, which makes the idea that one should "just take logs and use standard normal distribution methods” problematic. Even if all data were positive, this added variation causes difficulties since on the log scale the difference between 0.0001 and 0.001 is the same as the difference between 0.1 and 1.0, whereas in real-life exposure terms these are not at all the same. The distributions of the sample preparation and analytical variation components are nicely modeled using normal (not lognormal) distributions; see "What is a Beryllium Measurement? A Critical Look at Beryllium Quantitation” (C. B. Davis, D. Field, J. Hess, and D. Jensen, presented at Beryllium Particulates and Their Detection, Salt Lake City, UT, 2005).

Distributions of Be measurements are thus neither lognormal nor normal in general, but rather follow distributions with two variance components, one of each type. There are two limiting cases. When little Be is present, the minor lognormal component itself looks nearly normal, as does the overall measurement distribution. At the opposite extreme, the sampling and analytical 
error component becomes negligible and the lognormal component predominates. Most often, though, the bulk of the data is nearly normally distributed around or close to zero, with an upper tail of somewhat higher measurements.

A more accurate model for the actual distribution of measurements is described in "A Model for Measurements of Lognormally Distributed Environmental Contaminants” (C. B. Davis, D. Field, and T. E. Gran, presented at the Joint Statistical Meetings in Washington, D.C., 2009). This model may be viewed as an extension of the D. M. Rocke and S. Lorenzato model ("A TwoComponent Model for Measurement Error in Analytical Chemistry,” Technometrics 37, 1995). It is as yet intractable for making UTL- or PLall-type decisions directly, but is useful for testing the performance of UTL and PLall procedures such as those used in this study and others that have been proposed.

Most often data of this sort are censored, which means reporting all values less than some reporting limit $(\mathrm{RL})$ as simply “ $<\mathrm{RL}$." When data are censored, one is generally unaware of the actual distributions of the low-level measurements that typically form the majority of measurements from most facilities.

There are three possible statistical approaches: (1) one can use nonparametric statistical methods that rely only on the sample size and the upper-most data value(s); (2) one can use censored-data statistical methods such as those evaluated in "Parametric 95\%-95\% Upper Tolerance Limits for Censored Lognormal Data” (C. B. Davis, presented at the Joint Statistical Meetings in Seattle, WA, 2006); or (3) one can use uncensored-data methods when available.

We note that when data are censored, the distribution question does not arise so long as nonparametric statistical methods are used. However, as discussed by Davis and Grams ("When Laboratories Should Not Censor Analytical Data, and Why”, C. Davis and N. Grams, U.S. Environmental Protection Agency 25 ${ }^{\text {th }}$ Annual Conference on Managing Environmental Quality Systems, Austin, TX2006), in many cases this practice leads to high sample-size requirements and inefficiencies. The reason for this is that for clean facilities, in particular, the non-detect proportions tend to be high, and therefore nonparametric statistical methods with their higher sample-size requirements are needed. Using nonparametric statistical methods, the PLall approach is simply not available for any reasonable sample size; see "Simultaneous Nonparametric Prediction Limits” (with discussion and response, C. B. Davis and R. J. McNichols, Technometrics Vol. 41, pp. 89-112, 1999). The minimum sample size for $95 \%-95 \%$ UTLs is $n=59$. The irony is that the cleaner the facility, the more likely the need for larger sample sizes under this approach.

It turns out that even when there are enough “detects” to use the censored-data lognormal methods presented by Davis (2006, see above), the distortion implicit in using the lognormal model can reduce the statistical power considerably. Paradoxically, this loss of efficiency is greater when the RL is lower; the mechanism seems to be that implicitly assigning all the negative values to the interval between zero and the RL, creates a much more peaked (and hence, long-tailed) fitted model than is appropriate for the actual data, as discussed by Davis, Field, and Gran (2009, see above). We prefer to deal with the uncensored data. 
The results reported in this appendix address these issues in two steps. The first is to examine the distributions of measurements of blank wipe samples. The second is to develop and validate a heuristic approach for dealing with the impact of the lower tail of the distribution on the decision-making process.

\section{Distributions of Blank Measurements}

\section{DataChem}

The DataChem blanks are the most interesting. These are shown in the following plot, colorcoded by instrument and material. DataChem used two instruments, "G" and " $H$," and two types of wipe material (ghost wipes and Whatman filters) during different periods. There was generally no discernable difference between the distributions of Whatman filter blank measurements, with the critical exception of those from instrument $G$ during September 2002, which show a pronounced negative bias. There is a milder negative bias for ghost wipe blanks, but no statistically significant difference between instruments. (Where the instrument is given as "DC," the instrument used is not known.)

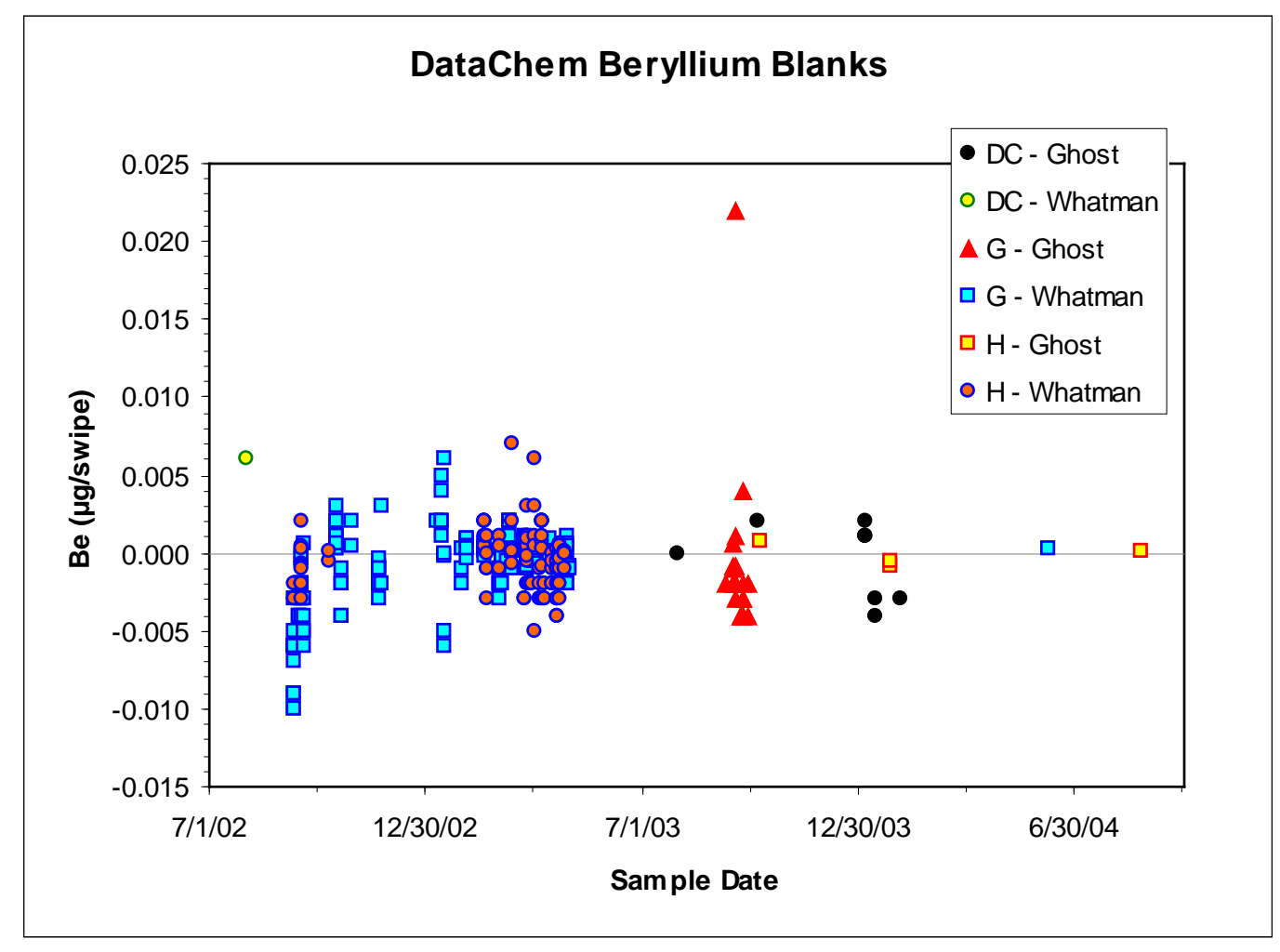

We have three periods:

September 2002, October 2002 through May 2003, and July 2003 through January 2004. These are the

\begin{tabular}{|clcccc|}
\hline Group & \multicolumn{1}{c}{$\begin{array}{c}\text { DataChem Blanks } \\
\text { Description }\end{array}$} & n & Mean & StDev \\
\hline A & Instrument G, Whatman Filter, September 2002 & 36 & -0.0041 & 0.0026 \\
B & Other Whatman Filter (through May 2003) & 227 & -0.0003 & 0.0018 \\
C & Ghost Wipe (starting July 2003) & 32 & -0.0013 & 0.0022 \\
\hline
\end{tabular}


sampling dates; analysis dates are assumed to follow promptly. Three samples obtained outside these time periods are excluded from the analysis, as is the outlier. Excluding the "DC" samples, there were originally six groups (period A ghost, etc.). Groups whose mean values were not statistically significantly different were combined. Means and standard deviations for each of the resulting three groups are given in the table; dot plots follow.

These distributions are very nearly normal; probability plot correlation coefficients (ProbPlot Rs) are $0.979,0.983$, and 0.991 for the three groups, respectively. The ProbPlot R for the pooled, centered data is 0.977 .

The negative bias in Group A was enough to make the large majority of measurements obtained in some critical buildings in the B Complex in North Las Vegas (NLV) negative. More importantly, both

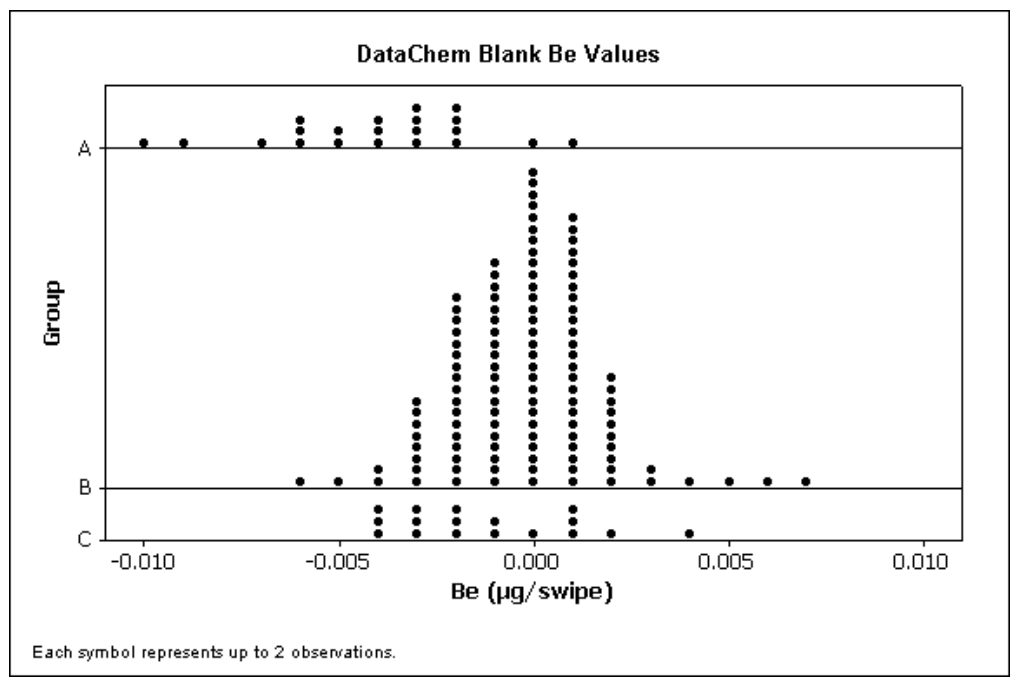
instruments were used in the analyses for two of those which were sampled during September 2002. For these reasons, it was decided to subtract the appropriate blank mean from each observation before proceeding with the statistical analysis; this is termed "blank correction." ("BC" in the tables in Appendix 2 is the negative of the blank mean.) Doing otherwise would artificially increase the variability seen in very low measurements; recall that when using lognormal models small relative variation in low measurements can have the same effect as large relative variation in higher measurements. In particular, such spurious variation in low-level measurements can have the adverse effect of inflating UTLs, PLalls, and so on.

For some facilities, work environment data obtained prior to September 2002 were used. These facilities had been occupied, and it was considered appropriate to include them in the study. They had been vacated by the time the systematic survey started, however. The data for these facilities were reported using the standard conventions regarding RLs and "non-detects”; accordingly, no low-level bias correction is available for these facilities, and our analyses use the censored-data lognormal approach recommended by Davis (2006, see above).

\section{Bechtel Nevada (BN) Adjusted}

The story is considerably different with the BNadj (see Appendix 3) blank data. Whether adjusted or not, these data show a considerable positive bias. The data were examined to determine whether there are any systematic relationships between the type of facility or the Nevada Test Site (NTS) area in which the blank was “obtained," but no such relationships were found ( $p$-values were 0.549 and 0.223 , respectively). There were three high outliers, as seen in the following plot; these were deleted from the statistical summary. 
It should also be noted that the iron (Fe) peak heights were atypically elevated in some field blank wipes associated with areas where Fe would be expected, particularly Area 1, 6, and 23 shops involving metal work. The Fe peak height exceeded the cutoff value of $65 \mathrm{~K}$ in two of these (see the Appendix 3 discussion). Again, though, no systematic relationship could be determined.

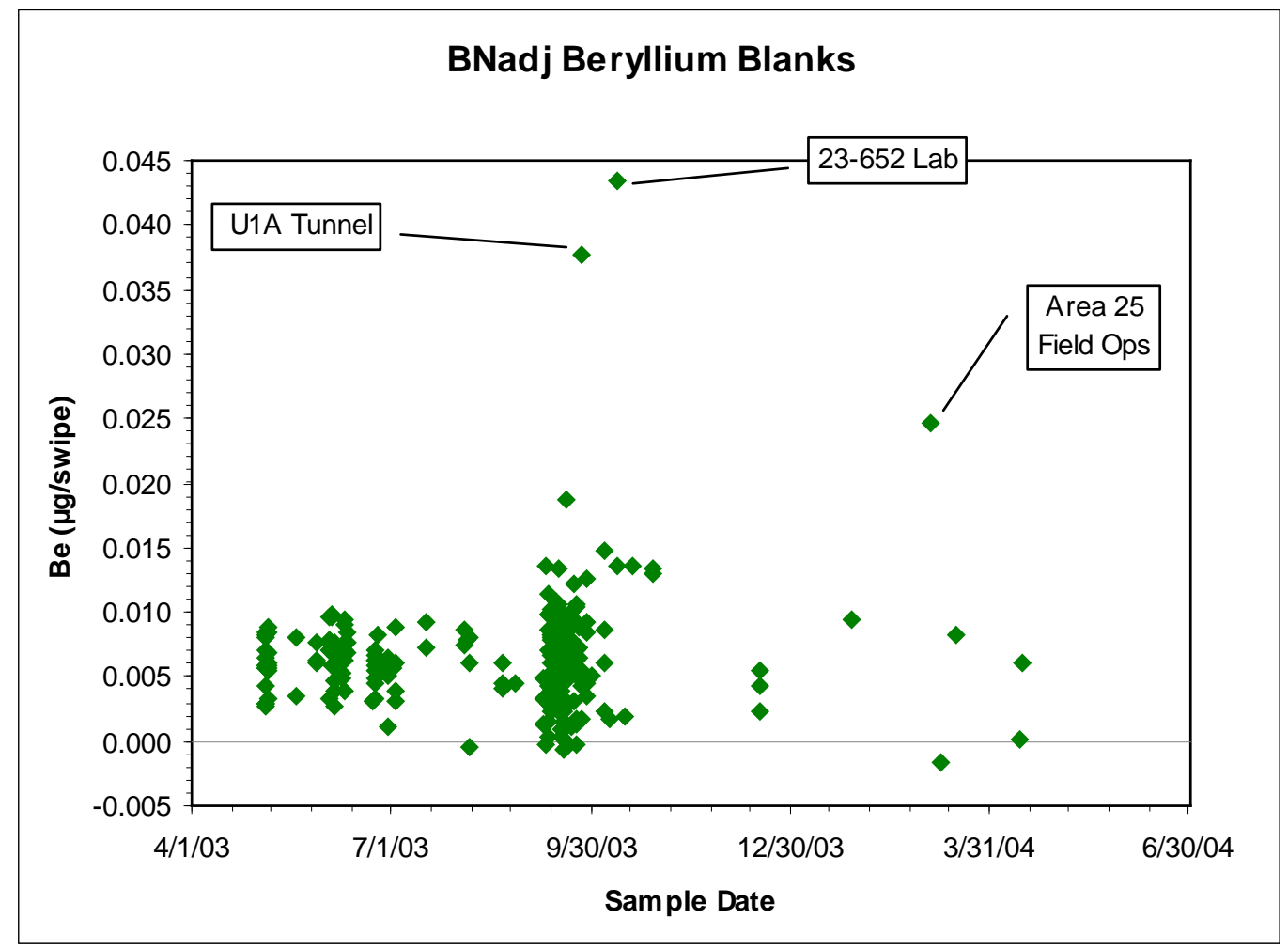

Excluding these three outliers, the distribution of BNadj blank values is as shown in the plot to the right and the table below. The normal distribution ProbPlot $\mathrm{R}$ is 0.992.

\begin{tabular}{|cll|}
\hline \multicolumn{3}{|c|}{ BNadj Blanks } \\
$\mathbf{n}$ & Mean & StDev \\
\hline 218 & 0.0062 & 0.0032 \\
\hline
\end{tabular}

For comparability with DataChem data at the low end of the distribution, a BC of (-0.0062) is added to BNadj data; doing so is

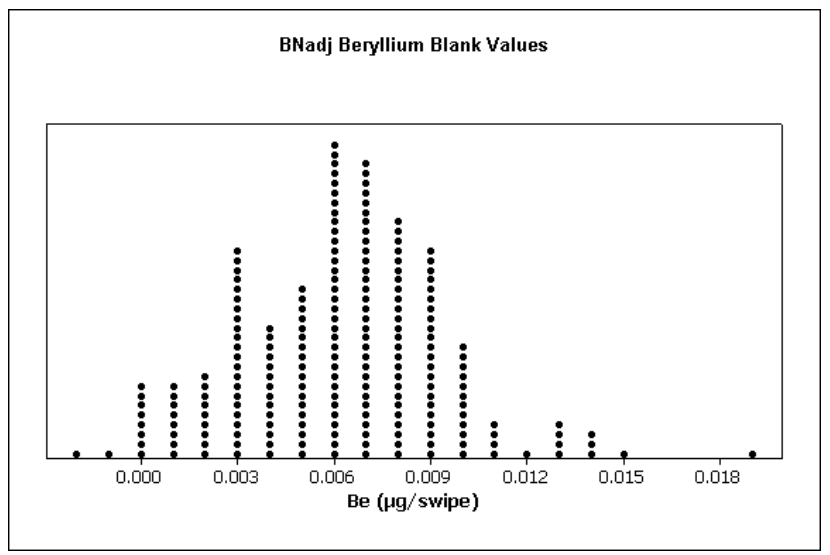
particularly important for those facilities with both DataChem and BNadj data.

\section{MS Analyses of Archived Samples}

As discussed in Appendix 3, in many samples analyzed by BN using its ICP-AES instrument, the Fe peak was too high to allow one to comfortably use the adjustment procedure. These samples were archived (retained as digestates) and reanalyzed quite some time later by ICP-MS. These 
analyses, denoted MS_arch, appear to be quite conservative as discussed in Appendix 3. Accordingly, if using the MS_arch data would allow a facility to "pass" the statistical test, this result was accepted.

The MS_arch blank data are shown in the next table and dot plot.

\begin{tabular}{|ccc|}
\hline \multicolumn{3}{|c|}{ MS_arch Blanks } \\
$\mathbf{n}$ & Mean & StDev \\
\hline 38 & -.0004 & 0.0011 \\
\hline
\end{tabular}

The normal probability plot correlation is only 0.850 with the outlier included. With that omitted, it is 0.960 . The mean used for the blank correction omits the outlier.

\section{$\underline{\text { MS Analyses of New Samples }}$}

The ad hoc approach of using the conservative MS_arch results was unsuccessful with a number of facilities. These were resampled during February 2008 (06-908) and January 2009 (numerous facilities; see Appendix 2). These data are denoted MS_new. The adjacent table and dot plot show the blank data associated with these samples and analyses.

\begin{tabular}{|cccc|}
\hline & \multicolumn{2}{c|}{ MS_new Blanks } & \\
& n & Mean & StDev \\
\hline Feb-08 & 8 & -0.0035 & 0.0013 \\
Jan-09 & 53 & -0.0001 & 0.0007 \\
\hline
\end{tabular}

There are eight outliers in the January 2009 blank data which are omitted from the mean calculation. Including the outliers the normal probability plot correlation is only 0.798 ; without them it is 0.978 .
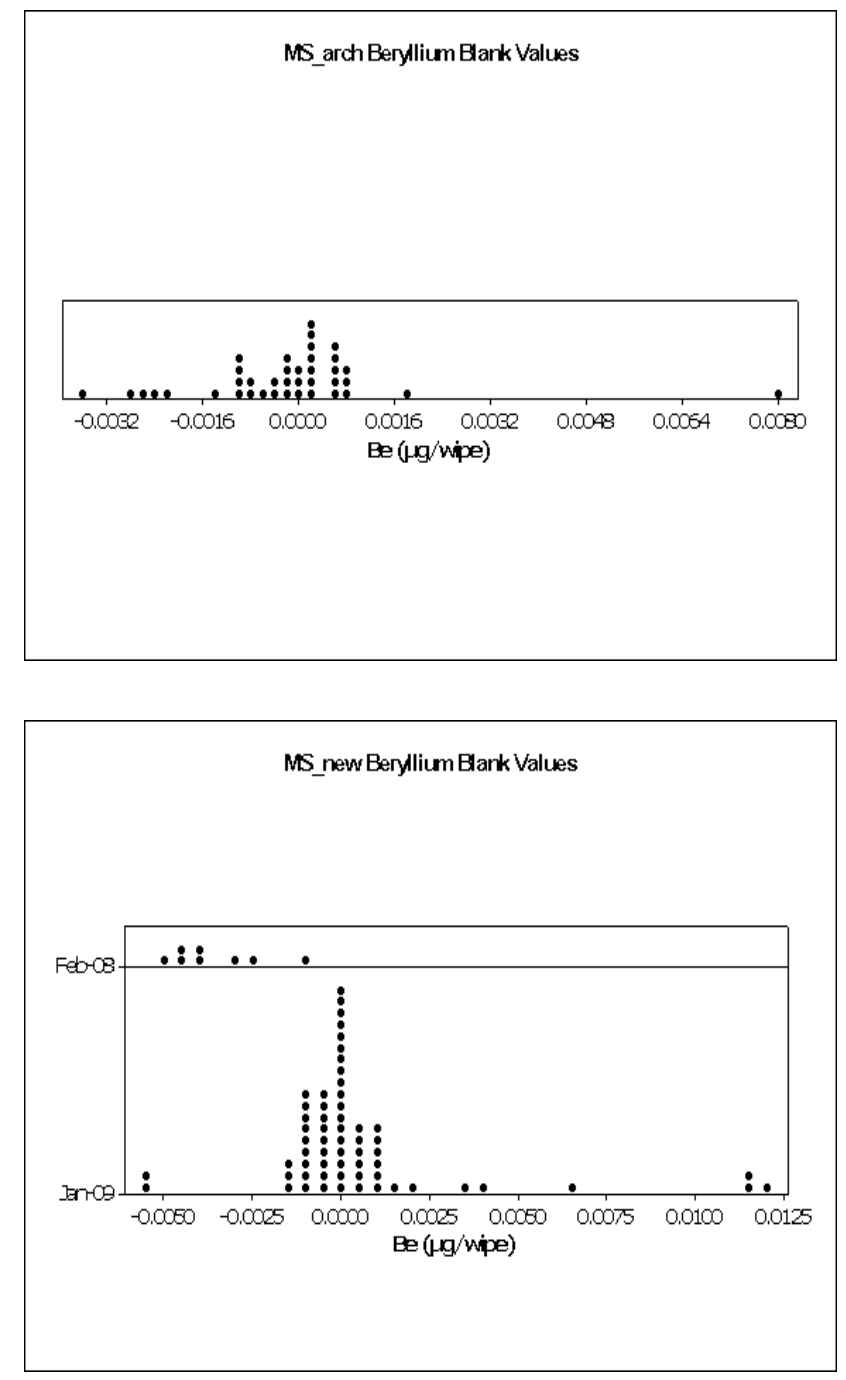

\section{Dealing with Negative Values}

The heuristic adopted for dealing with negative values is to add a small constant (delta) to each measurement, compute PLall and/or UTL with the resulting positive numbers using standard lognormal methods, and then subtract delta from the result. There are two issues: determining an appropriate value of delta; and demonstrating that the resulting shifted distribution is adequately lognormal. It is well known that probability distribution tests require moderate to large

\begin{tabular}{|cl|}
\hline Type & Description \\
\hline CO & Communications facility \\
EX & Experimental facility \\
FO & Field Operations facility \\
LA & Laboratory \\
OF & Office facility \\
QU & Quarters, including cafeteria \\
SH & Shop \\
ST & Storage \\
\hline
\end{tabular}


samples for reliability, whereas the data from many of our facilities contain fairly small numbers of IWA maxima, particularly, or Work Area (Area) values. The solution is to pool data across similar facilities, similar being defined in terms of facility use. Recall the codes used in Appendix 2, shown in the table on the previous page. Facility location (NTS area) was also considered as a factor in determining delta, but eventually discarded.

\section{$\underline{\text { DataChem }}$}

Based on comparisons of DataChem distributions and sample sizes, the following groupings were decided upon. This table contains also the delta and probability plot correlations (R and R90) for each group; these will be discussed presently. A very small number of high and low outliers are omitted in these analyses.

\begin{tabular}{|clcccc|}
\hline \multicolumn{6}{|c|}{ Type of Analysis } \\
& Facility Types & N & delta & R & R90 \\
\hline \multirow{2}{*}{ IWA } & OF QU & 513 & 0.0050 & 0.961 & 0.988 \\
& CO EX LA & 99 & 0.0020 & 0.893 & 0.971 \\
& FO SH ST & 165 & 0.0030 & 0.974 & 0.976 \\
\hline \multirow{2}{*}{ Area } & OF QU & 132 & 0.0020 & 0.932 & 0.969 \\
& EX & 47 & 0.0070 & 0.945 & 0.925 \\
& FO SH ST & 779 & 0.0055 & 0.955 & 0.986 \\
\hline
\end{tabular}

As an example of the working of this heuristic method, consider the data from OF [office facility] and QU [quarters] facility types. After adding the low-level shifts for blank correction, the distribution of the pooled IWA maximum data is shown in the plot at the right. The minimum value is -0.0039 .

We wish to avoid having low values too near zero after adding delta; a rule of thumb seems to be that after adding delta the minimum value should not be less than approximately 0.001 , and certainly never negative, after removing obvious outliers.

In this example, the minimum delta should therefore be around 0.0050 or so. For illustration, we try values starting at 0.0040 , increasing in increments of 0.0005 . A lognormal probability plot is constructed for each candidate delta value. The ideal for such a plot is a straight line; the probability plot correlation (ProbPlot R) measures the straightness of the line. With a perfect straight line $\mathrm{R}=1$. Because there are sporadic high

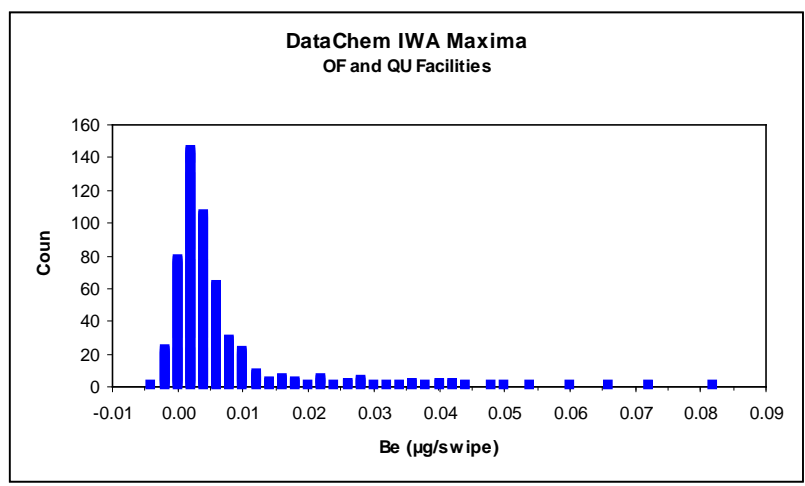
measurements in some facilities, the probability plot correlation of the lowest $90 \%$ of measurements (R90) is also taken into consideration. 
Clearly the value of delta has little effect on the high values, which in this example range up to 0.0811 . With the smallest delta candidates (0.0040 and 0.0045), the lowest values become excessively spread away from the body of the distribution, whereas with the larger candidate values the distribution tends toward a general concave appearance, indicating that the transformed data are still right-

\begin{tabular}{|ccc|}
\hline delta & R & R90 \\
\hline 0.0040 & 0.959 & 0.954 \\
0.0045 & 0.965 & 0.982 \\
$\mathbf{0 . 0 0 5 0}$ & $\mathbf{0 . 9 6 1}$ & $\mathbf{0 . 9 8 8}$ \\
0.0055 & 0.956 & 0.980 \\
0.0060 & 0.952 & 0.991 \\
0.0065 & 0.947 & 0.991 \\
\hline
\end{tabular}
skewed. In this example, the highest R of 0.965 is obtained with delta $=0.0045$, but R90 is still increasing at that point. The next trial value (delta $=0.0050)$ is selected as a compromise.

The lognormal probability plot (LN ProbPlot) for the selected delta (left below) shows some deviation from the ideal straight line, but no consistent downward curvature at the upper tail and, more importantly, no lower-tail outliers, both of which are of concern for PLall and UTL inferences. The fitted shifted lognormal distribution using delta $=0.0050$ is shown in the plot on the right below.
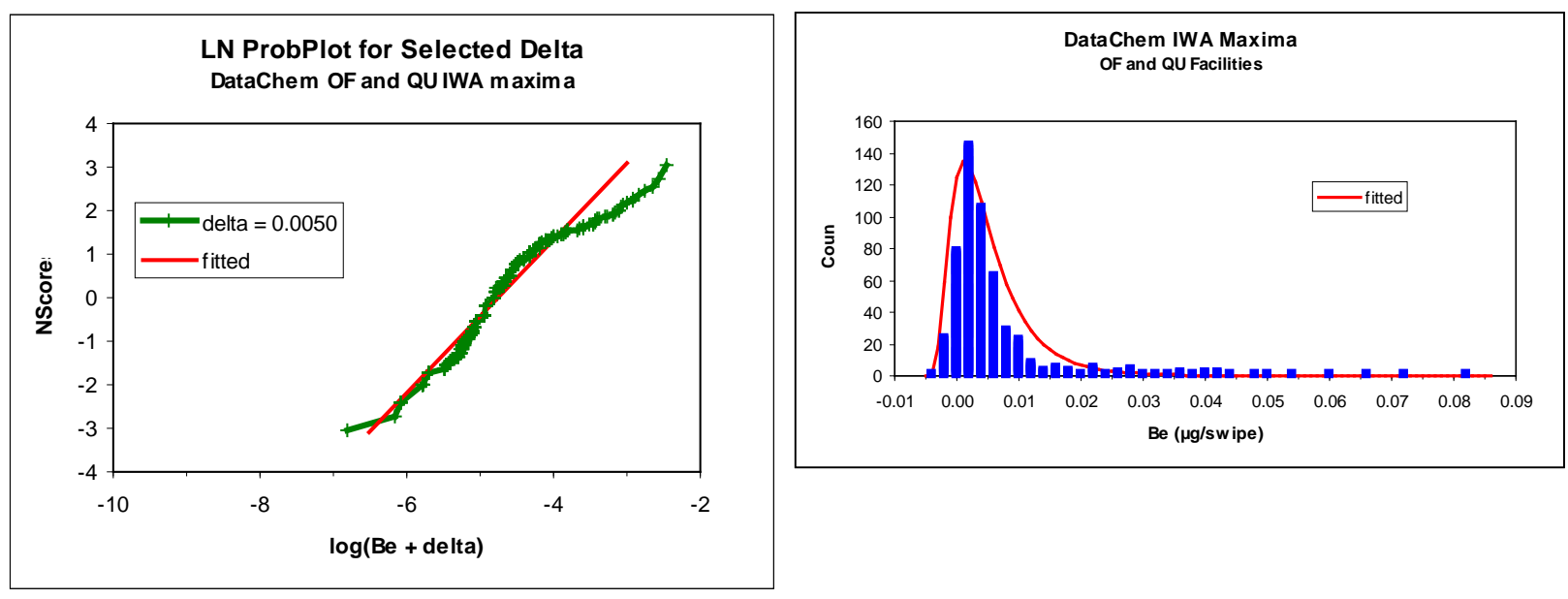

Incidentally, for this example, the normal (not lognormal) ProbPlot R is 0.746, decidedly lower than acceptable. As delta increases, the transformed data ProbPlot R decreases toward this value, and the UTL and PLall values decrease toward those that would be obtained using normal distribution methods without data transformation. In the delta selection reported here, the preference is for the smallest delta values that provide adequately high R and R90 correlations.

\section{$\underline{\text { BN Adjusted }}$}

These data are actually a mixture of BNadj data with some DataChem data, all data being blank-corrected as discussed previously. The latter are included for one of three reasons: (a) DataChem side-by-side values were used where the BN sample Fe peak height was excessive (see Appendix 3), (b) DataChem side-by-side values were used when they are higher than the BNadj values, and (c) in a couple of instances initial small data sets of samples analyzed by DataChem were augmented by additional samples with BNadj analyses. 


\begin{tabular}{|clcccc|}
\hline \multicolumn{6}{|c|}{ Type of Analysis } \\
& Facility Types & N & delta & R & R90 \\
\hline \multirow{2}{*}{ IWA } & OF QU & 238 & 0.0050 & 0.943 & 0.971 \\
& EX LA & 48 & 0.0020 & 0.987 & 0.984 \\
& FO SH ST & 54 & 0.0030 & 0.976 & 0.991 \\
\hline \multirow{2}{*}{ Area } & EX LA & 75 & 0.0070 & 0.989 & 0.992 \\
& FO & 105 & 0.0080 & 0.988 & 0.989 \\
\hline
\end{tabular}

For the most part, the delta values selected in the previous section for DataChem will work nicely with the BNadj data. The exception is with FO [Field Operations] Area measurements, as seen in the adjacent table.

\section{$\underline{\text { BN Adjusted Along with BN-MS Archived Sample Analyses }}$}

As discussed elsewhere, there are a number of measurements with high Fe peak heights that preclude the adjustment described in Appendix 3. The digestates from these analyses were archived. These archived digestates were reanalyzed during January-April 2007 using a newly acquired ICP-MS instrument, giving the MS_arch data. As discussed in Appendix 3, there is concern about the quality of these data, given the excessive holding time involved. Many of the MS_arch values seem rather high; this could be consistent with either concentration of the digestate via evaporation while it was archived or higher Be concentrations accompanying the higher Fe concentrations in some areas of some facilities. For the purposes of this report, it was decided to use the MS_arch values where possible, recognizing that they may be biased high and, therefore, result in conservative analyses or decisions. For facilities for which this stratagem did not provide satisfactory results, a supplemental round of sampling was conducted during January 2009 (February 2008 for 06-908), with fresh samples analyzed by ICP-MS (MS_new data); see the discussion to follow.

The data sets that result include a mixture of MS_arch, BNadj, and DataChem data. Accordingly, deltas for these data sets are determined separately from those for only BNadj and/or DataChem. These are given in the table at the right.

\section{$\underline{\text { Shift analyses for cluster sampling }}$}

An alternate analysis of the IWA data uses all the values, not just the IWA maxima, as discussed in the following section. The delta values should be expected to be larger than those for the IWA maxima, since the distribution of all IWA data values includes lower values. Two versions are done: one for those facilities with data from only DataChem and the other for facilities with BNadj data and possibly also some DataChem and/or MS_arch data.

\begin{tabular}{|c|c|c|c|c|c|}
\hline & Mixed BNa & d Ms & ch delta & ction & \\
\hline Type 0 & nalysis & & & & \\
\hline & Facility Types & $\mathbf{N}$ & delta & $\mathbf{R}$ & R90 \\
\hline IWA & OF QU & 84 & 0.0040 & 0.969 & 0.966 \\
\hline & EX LA & 80 & 0.0040 & 0.958 & 0.949 \\
\hline & FO SH ST & 34 & 0.0030 & 0.969 & 0.975 \\
\hline Area & LA & 52 & 0.0070 & 0.974 & 0.969 \\
\hline & FO SH ST & 975 & 0.0080 & 0.980 & 0.979 \\
\hline
\end{tabular}

\begin{tabular}{|c|c|c|c|c|c|}
\hline & DataChe & a selec & for all I & data & \\
\hline Type & f Analysis & & & & \\
\hline & Facility Types & $\mathbf{N}$ & delta & $\mathbf{R}$ & R90 \\
\hline IWA & OF QU & 2618 & 0.0085 & 0.955 & 0.967 \\
\hline all & CO EX LA & 476 & 0.0040 & 0.949 & 0.972 \\
\hline & FO SH ST & 785 & 0.0055 & 0.929 & 0.963 \\
\hline & BNadj et & selec & for all I & data & \\
\hline Type & f Analysis & & & & \\
\hline & Facility Types & $\mathbf{N}$ & delta & $\mathbf{R}$ & R90 \\
\hline IWA & OF QU & 1675 & 0.0085 & 0.958 & 0.972 \\
\hline all & EX LA & 453 & 0.0080 & 0.982 & 0.987 \\
\hline & FO SH ST & 1718 & 0.0090 & 0.938 & 0.981 \\
\hline
\end{tabular}




\section{New ICP-MS analyses}

The table to the right shows the delta values for MS_new mixed with other analyses. There are relatively few data values, particularly for the IWA analyses, and most come from very similar environments.

\begin{tabular}{|cccccc|}
\hline \multicolumn{6}{|c|}{ Mixed MS_new and other delta Selection } \\
Type of Analysis \\
& Facility Types & N & delta & R & R90 \\
\hline IWA & OF FO ST & 28 & 0.0030 & 0.980 & 0.971 \\
\hline Area & FO SH ST & 651 & 0.0085 & 0.979 & 0.975 \\
\hline
\end{tabular}

\section{UTL analyses with cluster sampling data}

The sampling plans used for IWA facilities and portions of facilities are similar to cluster samples in surveys. A basic model for such measurements is

$$
X_{i j}=\mu+W_{i}+Z_{i j}
$$

where $\mu$ is the overall mean value for the facility, $X_{i j}$ is the $j^{\text {th }}$ measurement in the $i^{\text {th }}$ cluster (here, $i^{\text {th }}$ IWA), $W_{i}$ represents any random effect common to all measurements in cluster $i$, and $Z_{i j}$ represents the random variation in the measurements that are independent of those for other measurements. There are I clusters (IWAs) in all, and $\mathrm{J}_{i}$ measurements in the $i^{\text {th }}$ cluster. In this study, $\mathrm{J}_{i}$ is nearly always between 4 and 6 ; for various facilities, I can range from 2 to nearly 100.

We assume that data are normally distributed after appropriate transformation. In particular, the $W_{i}$ are independent, normally distributed random variables with mean 0 and standard deviation $\tau$; the $Z_{i j}$ are independent, normally distributed random variables with mean 0 and standard deviation $\sigma$; and the $Z_{i j}$ are independent of the $W_{i}$. The distribution of the $X_{i j}$ is then normal with mean $\mu$ and variance (squared standard deviation) $\left(\tau^{2}+\sigma^{2}\right)$, denoted as VAR in the following.

The following derivations are for $\mathrm{J}_{i}$ all the same $(=\mathrm{J})$, which is approximately true in this survey. The average of measurements for the $i^{\text {th }}$ cluster is $\bar{X}_{i}$, which has variance $\left(\tau^{2}+\sigma^{2} / \mathrm{J}\right)$, and the overall average of measurements is $\bar{X}_{\text {.. }}$, which has variance $\left(\tau^{2} / \mathrm{I}+\sigma^{2} / \mathrm{IJ}\right)$. We have the usual sums of squares between and within clusters from the Analysis of Variance (ANOVA):

$$
S S B=\mathrm{J} \sum_{\mathrm{i}}\left(\bar{X}_{i .}-\bar{X}_{. .}\right)^{2} \text { and } S S W=\sum_{i} \sum_{j}\left(X_{i j}-\bar{X}_{i .}\right)^{2},
$$

and the usual mean squares

$$
M S B=S S B /(\mathrm{I}-1) \text { and } M S W=S S W /(\mathrm{I}(\mathrm{J}-1))
$$

The sums of squares are proportion to independent chi-square random variates:

$$
S S B \sim\left(\mathrm{J} \tau^{2}+\sigma^{2}\right) \chi^{2}(\mathrm{I}-1) \text { and } S S W \sim \sigma^{2} \chi^{2}(\mathrm{I}(\mathrm{J}-1)) .
$$


To estimate VAR, we find an unbiased estimator which is a linear combination of MSB and $M S W$; this is

$$
\hat{\mathrm{VAR}}=\frac{1}{\mathrm{~J}} M S B+\frac{\mathrm{J}-1}{\mathrm{~J}} M S W .
$$

Being a linear combination of independent $\chi^{2}$ random variables, its distribution is approximately that of a multiple of a $\chi^{2}$ random variable having the right mean. The estimated approximate degrees of freedom are given by the Satterthwaite formula, which becomes

$$
\hat{\mathrm{DF}}=\frac{\left(\frac{1}{\mathrm{~J}} M S B+\frac{\mathrm{J}-1}{\mathrm{~J}} M S W\right)^{2}}{\frac{\left(\frac{1}{\mathrm{~J}} M S B\right)^{2}}{\mathrm{I}-1}+\frac{\left.\frac{\mathrm{J}-1}{\mathrm{~J}} M S W\right)^{2}}{\mathrm{I}(\mathrm{J}-1)}} .
$$

This simplifies to the satisfying formula

$$
\hat{\mathrm{D} F}=\frac{(F+\mathrm{J}-1)^{2}}{\frac{F^{2}}{\mathrm{I}-1}+\frac{\mathrm{J}-1}{\mathrm{I}}},
$$

where $F=M S B / M S W$ is the usual $F$ statistic from the ANOVA. Note that as $F$ becomes large $\hat{D F}$ approaches (I-1), which is the appropriate degrees of freedom for the case with predominant cluster-to-cluster variation, whereas for $F$ small (around 1) $\hat{D} F$ is close to its correct value (IJ-1) for the case with negligible cluster-to-cluster variation.

In the standard independent sampling situation, the UTL multiplier K is given by

$$
\mathrm{K}=\frac{1}{\sqrt{\mathrm{n}}} \mathrm{T}^{-1}(\gamma, \mathrm{DF}, \mathrm{DEL}),
$$

where $\mathrm{T}$ is the non-central $t$ distribution cumulative distribution function, $\gamma$ is the desired confidence level, $\mathrm{n}$ is the number of observations, and DF is the degrees of freedom (generally $\mathrm{n}-1)$. DEL $=\mathrm{z}_{\beta} \sqrt{\mathrm{n}}$ is the noncentrality parameter, where $\mathrm{z}_{\beta}$ is the $100 \mathrm{\beta}^{\text {th }}$ percentile of the standard normal distribution and 100ß is the desired percentile for the UTL. In this application $\beta=0.95$; $\gamma$ is generally also 0.95 , except in cases where a facility is treated as consisting of two or more parts and we want the overall confidence level to be no less than $95 \%$, so the individual confidence levels are set higher (see Appendix 1).

The function of $\mathrm{n}$ in the derivation of the UTL K multiplier is to account for the variability of the mean. In the standard situation, without cluster sampling, $\operatorname{Var}(\bar{X})=\sigma^{2} / \mathrm{n}$. With cluster sampling, though, $\operatorname{Var}\left(\bar{X}_{\text {.. }}\right)=\frac{1}{\mathrm{I}} \tau^{2}+\frac{1}{\mathrm{IJ}} \sigma^{2}$. As a first approach to accounting for this variation, 
we derive a pseudo-sample size $N^{*}$ by setting $\frac{1}{N^{*}}\left(\tau^{2}+\sigma^{2}\right)=\frac{1}{\mathrm{I}} \tau^{2}+\frac{1}{\mathrm{IJ}} \sigma^{2}$. Solving for $N^{*}$ and substituting their estimators for the unknown parameters $\tau^{2}$ and $\sigma^{2}$, leads to the satisfying formula

$$
N^{*}=\mathrm{I}(1+(\mathrm{J}-1) / F)
$$

Note that for $F$ large this approaches I, as it should if the analytical variation is negligible relative to between-cluster variation, and if $F \sim 1$ this is just IJ, the total number of measurements, as is appropriate if cluster-to-cluster variation is negligible.

If $F$ is 1 or less, a standard UTL analysis is used with no allowance for a cluster effect.

Unfortunately, between this approximation and the Satterthwaite approximation the actual confidence level is a bit too variable, as was discovered during Monte Carlo simulations. In particular, for small I (fewer than 10 IWAs) and large $\tau / \sigma$, the confidence was lower than desired. (In preliminary analyses, $\tau / \sigma$ was found to range from 0 to somewhat less than 1 for our data.) Accordingly, three UTL multipliers are formed:

K1 uses $N^{*}$ and $\hat{D F}$;

K2 uses I and $\hat{D F}$; and

K3 uses I and (I-1) for the sample size and degrees of freedom, respectively.

K2 and K3 are conservative. Weighted averages of these, with weights depending on the number of IWAs I, are found to maintain the desired confidence level reasonably well (between $93 \%$ and $97 \%$ for $0 \leq \tau / \sigma \leq 1)$ :

$$
\begin{array}{ll}
\mathrm{K} 12 & =\mathrm{a} \mathrm{K} 1+(1-\mathrm{a}) \mathrm{K} 2 \text { for } \mathrm{I} \geq 10, \text { and } \\
\mathrm{K} 123 & =0.5 \mathrm{~K} 1+0.5(\mathrm{~K} 2+\mathrm{K} 3) / 2 \text { for } \mathrm{I}<10, \text { where } \\
\mathrm{a} & =0.6-1 / \mathrm{I} .
\end{array}
$$

The Monte Carlo simulations used $\mathrm{J}=5$, being approximately the average number of measurements taken per IWA.

The formulas for the unbalanced case ( $\mathrm{J}_{i}$ not all the same) are considerably more complicated. An adequate approximation is obtained, though, using the variance component estimates and $F$ ratio obtained from the data using standard commercial statistical software.

\section{Analysis of Soil and Bulk Samples and Metal Ratios}

As discussed in Appendix 1, applying the DOE RC with UTLs (or PLalls) for facility surveys is borrowed from the Title 10 Code of Federal Regulations Part §850.31(b)(1) provisions for acceptable surface contamination levels associated with items to be released to the public. That regulation allows an alternate comparison with soils near the equipment or items involved. To apply this alternate comparison to facility surveys, one obtains soil samples outside the facility and bulk material samples inside and compares the Be concentrations in those samples. 
The regulatory provision is clearly intended to avoid deciding that equipment is "dirty" and in need of cleanup simply due to naturally occurring Be in the soils. Accordingly, in pursuing this mode of decision-making, National Security Technologies, LLC, has extended this approach to include evaluation of metal ratios. The ICP-MS analyses used most recently in the January 2009 resampling of certain facilities allow for determination of several other metals that are found naturally in the soils at the NTS and ancillary facilities. Those metals are $\mathrm{Co}, \mathrm{Ni}, \mathrm{Cu}, \mathrm{Y}, \mathrm{Nb}$, and ${ }^{238} \mathrm{U}$.

The approach adopted is the following. Small numbers of bulk samples were obtained inside most of the facilities involved in the January 2009 resampling, along with exterior soil samples; theses were analyzed for the additional metals. In addition, any wipe sample whose Be concentration exceeded the DOE RC of $0.2 \mu \mathrm{g} / 100 \mathrm{~cm}^{2}$ [micrograms per square centimeters] was also analyzed for those additional metals. Typical intervals for the Be soil concentrations were obtained; those are shown graphically below. Also, typical intervals for logs of the ratios were obtained.

The draft DOE Technical Standard does not at this time contain a protocol for comparisons between bulk and soil concentrations or for comparing metal ratios. For the purposes of this study, these comparisons are used to supplement comparisons of surface concentrations with the DOE RC in cases where a facility does not "pass" using the RC comparisons. The Be concentrations in bulk samples with high Be concentrations were compared with metal ratios found in exterior soils. Where the Be concentrations in interior bulk samples and/or the metal ratios in bulk or wipe samples were consistent with those observed in exterior soils, the Be present in the facility was taken to be of natural origin and, therefore, not "contamination" in the sense of 10 CFR 850 . Facilities judged not contaminated based on this evidence are designated by blue coding in the chart in Appendix 2.

\section{Be soil data}

The plot at right shows the distribution of all 37 soil Be values. The fitted middle $95 \%$ of these data extends from 0.38 to $1.86 \mathrm{mg} / \mathrm{Kg}$ [milligrams per kilograms]. There is some indication that the soil values may vary systematically from NTS Area to Area, as seen in the next plot.

In that plot, " $\mathrm{N}$ " indicates soil samples obtained in Areas 1, 3, and 12 to the north of the NTS; "C" indicates those obtained in Areas 5 and 6; " $S$ " indicates those obtained in Areas 23, 25, 26, and 27; and "NLV" indicates those obtained in NLV or Nellis Air Force Base (AFB). Differences in means among these locations are highly statistically significant $(p=0.001)$, with NLV being clearly lower than $\mathrm{N}$ and $\mathrm{S}$, and $\mathrm{N}$ being somewhat higher than $\mathrm{C}$.

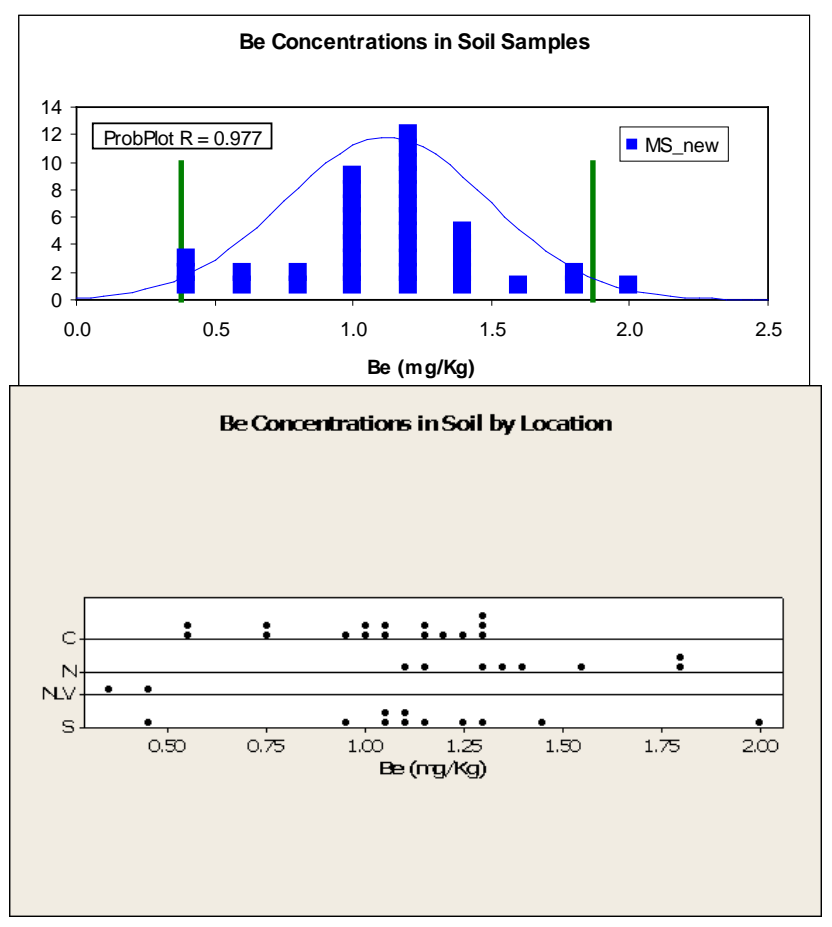


These data are mildly consistent with soil samples obtained previously from Frenchman Flat, Jackass Flat, and Yucca Flat, and analyzed by Y-12 using ICP-AES. The middle 95\% of those data extend from 0.20 to $1.28 \mathrm{mg} / \mathrm{Kg}$; the areas sampled correspond to some areas labeled "C" and "S" in the adjacent dot plot. Differences in sample preparation methods are surely involved as well.

\section{$\underline{\text { Metal Ratio Data }}$}

The idea here is that natural Be in soils is associated with other metals, which can also be analyzed via ICP-MS. If that association is reasonably consistent, the value of the ratio of the concentrations can provide supporting evidence that the Be concentration in a bulk or wipe sample is consistent with what one would expect based on, for example, the $\mathrm{Y}$ or $\mathrm{Nb}$ concentration in that sample. This discussion is carried out in terms of logs of ratios, as those tend to have more symmetric distributions.

In each case, Be is the denominator of the ratio. Therefore, ratios with Be relatively high compared with the other metal(s) will have relatively low values; we will be less concerned about ratios that are relatively high. Here are plots of the distributions of these log ratios in soil data, with the middle $95 \%$ of the distributions indicated.
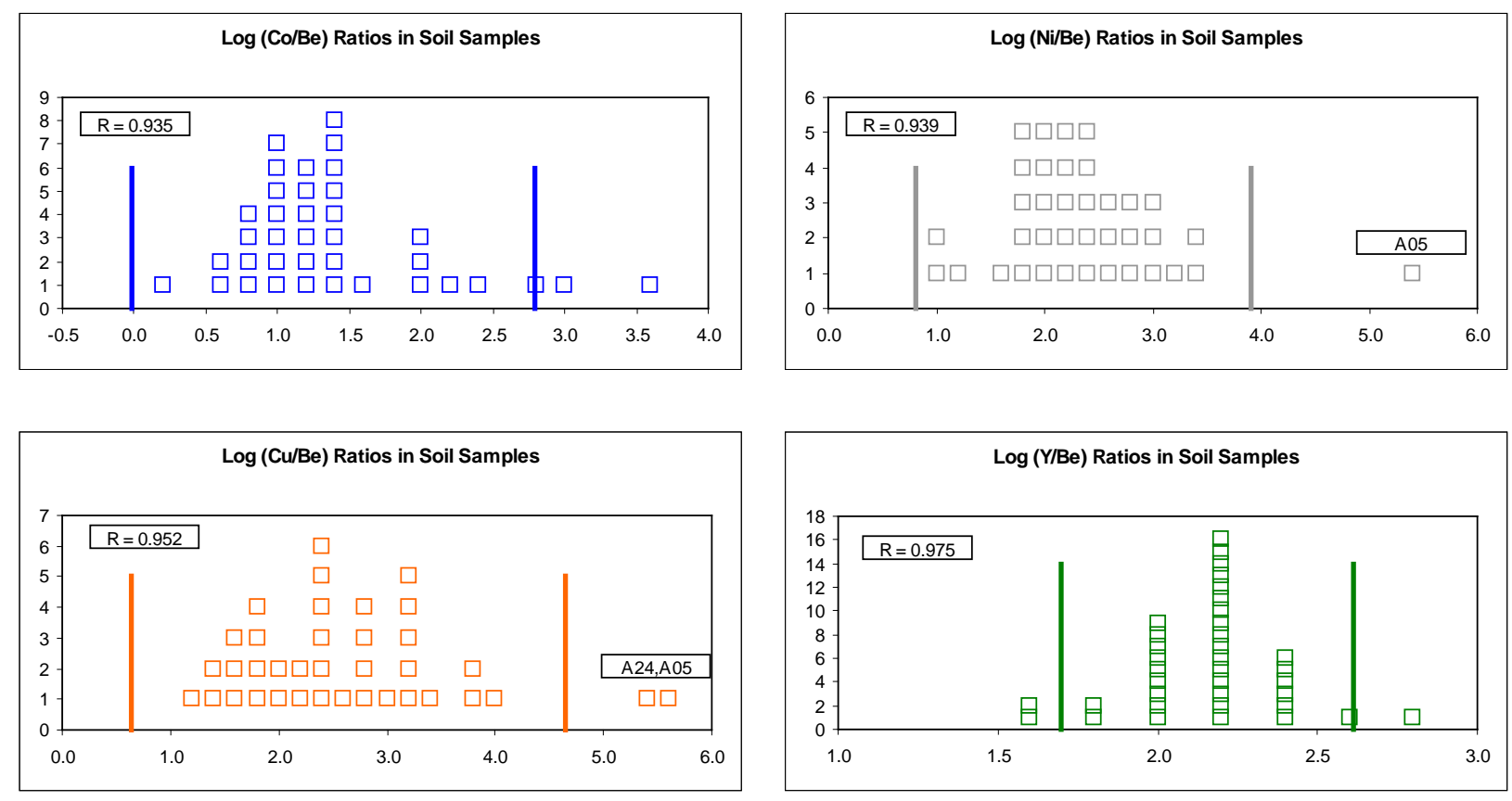

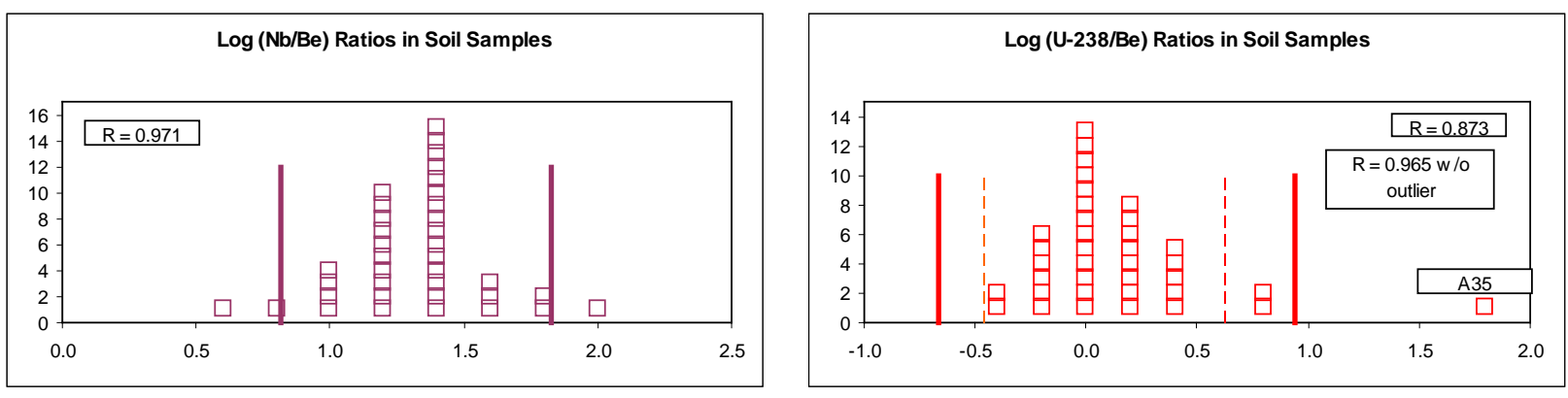

There are a few outliers in these plots with relatively low Be; those are indicated by Area. The normal probability plot correlation $\mathrm{R}$ is given in each plot; for $\mathrm{U}$, where this is less than 0.9 , computations are repeated with the outlier from Nellis AFB (with low Be compared with $U$ ) omitted.

The next table lists the means and standard deviations of the ratios along with their middle 95\% intervals. The one after that gives the results of an ANOVA, testing whether the means of the ratios are the same from location to location, using the same location groups as for the Be soil concentrations themselves. Referring to both tables, we find in particular that $\mathrm{Ni} / \mathrm{Be}$ is quite variable throughout the NTS (standard deviation is relatively large), but no systematic differences among location groups are found. For $\mathrm{Co}$ and $\mathrm{Cu}$, the ratios are also quite variable (standard deviation $=0.70$ and 1.00 , respectively); the statistically significant differences among locations contrast the NLV locations with relatively low Be with one or all of the remaining groups of locations.

The other three ratios have rather lower standard deviation values, and hence will provide better evidence for consistency of Be with the other metals involved. Y/Be has the lowest standard deviation (0.23), and does not quite have statistically significant differences among location groups. $\mathrm{Nb} / \mathrm{Be}$ and $\mathrm{U} / \mathrm{Be}$ are next best; the U/Be ratios are different in NLV because of the relatively low Be found in the soil samples there.

\begin{tabular}{|ccccc|}
\hline \multicolumn{5}{c}{ 95 Percent Intervals for log Metal Ratios } \\
Metals & Mean & StDev & Lower & Upper \\
\hline $\mathrm{Co} / \mathrm{Be}$ & 1.39 & 0.70 & -0.02 & 2.80 \\
$\mathrm{Ni} / \mathrm{Be}$ & 2.36 & 0.78 & 0.81 & 3.91 \\
$\mathrm{Cu} / \mathrm{Be}$ & 2.64 & 1.00 & 0.64 & 4.65 \\
$\mathrm{Y} / \mathrm{Be}$ & 2.15 & 0.23 & 1.70 & 2.61 \\
$\mathrm{Nb} / \mathrm{Be}$ & 1.32 & 0.25 & 0.81 & 1.83 \\
$\mathrm{U} / \mathrm{Be}$ & 0.14 & 0.40 & -0.67 & 0.94 \\
$\mathrm{U} / \mathrm{Be}^{*}$ & 0.09 & 0.27 & -0.46 & 0.63 \\
\hline
\end{tabular}

\begin{tabular}{|ccc|}
\hline \multicolumn{3}{|c|}{ Tests of Mean Ratios Among Locations } \\
Metals & $\boldsymbol{p}$ & Pattern \\
\hline $\mathrm{Co} / \mathrm{Be}$ & 0.036 & $\mathrm{~N}<\mathrm{NLV}$ \\
$\mathrm{Ni} / \mathrm{Be}$ & 0.454 & \\
$\mathrm{Cu} / \mathrm{Be}$ & 0.007 & rest $<\mathrm{NLV}$ \\
$\mathrm{Y} / \mathrm{Be}$ & 0.062 & \\
$\mathrm{Nb} / \mathrm{Be}$ & 0.048 & $\mathrm{~S}<\mathrm{C}$ \\
$\mathrm{U} / \mathrm{Be}$ & 0.000 & rest $<\mathrm{NLV}$ \\
\hline
\end{tabular}

